\title{
Lectures on Symplectic Geometry
}

\author{
Ana Cannas da Silva ${ }^{1}$ \\ revised January 2006
}

Published by Springer-Verlag as

number 1764 of the series Lecture Notes in Mathematics.

The original publication is available at www.springerlink.com.

${ }^{1}$ E-mail: acannas@math.ist.utl.pt or acannas@math.princeton.edu 


\section{Foreword}

These notes approximately transcribe a 15-week course on symplectic geometry I taught at UC Berkeley in the Fall of 1997.

The course at Berkeley was greatly inspired in content and style by Victor Guillemin, whose masterly teaching of beautiful courses on topics related to symplectic geometry at MIT, I was lucky enough to experience as a graduate student. I am very thankful to him!

That course also borrowed from the 1997 Park City summer courses on symplectic geometry and topology, and from many talks and discussions of the symplectic geometry group at MIT. Among the regular participants in the MIT informal symplectic seminar 93-96, I would like to acknowledge the contributions of Allen Knutson, Chris Woodward, David Metzler, Eckhard Meinrenken, Elisa Prato, Eugene Lerman, Jonathan Weitsman, Lisa Jeffrey, Reyer Sjamaar, Shaun Martin, Stephanie Singer, Sue Tolman and, last but not least, Yael Karshon.

Thanks to everyone sitting in Math 242 in the Fall of 1997 for all the comments they made, and especially to those who wrote notes on the basis of which I was better able to reconstruct what went on: Alexandru Scorpan, Ben Davis, David Martinez, Don Barkauskas, Ezra Miller, Henrique Bursztyn, John-Peter Lund, Laura De Marco, Olga Radko, Peter Pribík, Pieter Collins, Sarah Packman, Stephen Bigelow, Susan Harrington, Tolga Etgü and Yi Ma.

I am indebted to Chris Tuffley, Megumi Harada and Saul Schleimer who read the first draft of these notes and spotted many mistakes, and to Fernando Louro, Grisha Mikhalkin and, particularly, João Baptista who suggested several improvements and careful corrections. Of course I am fully responsible for the remaining errors and imprecisions.

The interest of Alan Weinstein, Allen Knutson, Chris Woodward, Eugene Lerman, Jiang-Hua Lu, Kai Cieliebak, Rahul Pandharipande, Viktor Ginzburg and Yael Karshon was crucial at the last stages of the preparation of this manuscript. I am grateful to them, and to Michèle Audin for her inspiring texts and lectures.

Finally, many thanks to Faye Yeager and Debbie Craig who typed pages of messy notes into neat $\mathbb{A} T E X$, to João Palhoto Matos for his technical support, and to Catriona Byrne, Ina Lindemann, Ingrid März and the rest of the Springer-Verlag mathematics editorial team for their expert advice.

Ana Cannas da Silva

Berkeley, November 1998

and Lisbon, September 2000 


\section{Contents}

Foreword $v$

$\begin{array}{lr}\text { Introduction } & 1\end{array}$

I Symplectic Manifolds 3

1 Symplectic Forms 3

1.1 Skew-Symmetric Bilinear Maps . . . . . . . . . . . . . . . . . 3

1.2 Symplectic Vector Spaces . . . . . . . . . . . . . . . . . . . 4

1.3 Symplectic Manifolds . . . . . . . . . . . . . . . . . . . . 6

1.4 Symplectomorphisms . . . . . . . . . . . . . . . . . 7

Homework 1: Symplectic Linear Algebra $\quad 8$

2 Symplectic Form on the Cotangent Bundle 9

2.1 Cotangent Bundle . . . . . . . . . . . . . . . . . 9 9

2.2 Tautological and Canonical Forms in Coordinates . . . . . . . . . 9

2.3 Coordinate-Free Definitions . . . . . . . . . . . . . . . . . . 10

2.4 Naturality of the Tautological and Canonical Forms . . . . . . . . 11

Homework 2: Symplectic Volume 13

$\begin{array}{ll}\text { II Symplectomorphisms } & 15\end{array}$

3 Lagrangian Submanifolds 15

3.1 Submanifolds . . . . . . . . . . . . . . . . . . . 15

3.2 Lagrangian Submanifolds of $T^{*} X \ldots \ldots \ldots \ldots \ldots$

3.3 Conormal Bundles . . . . . . . . . . . . . . . . . . . 17

3.4 Application to Symplectomorphisms . . . . . . . . . . . . . 18

Homework 3: Tautological Form and Symplectomorphisms 20

4 Generating Functions 22

4.1 Constructing Symplectomorphisms . . . . . . . . . . . . . . 22

4.2 Method of Generating Functions . . . . . . . . . . . . . . 23

4.3 Application to Geodesic Flow . . . . . . . . . . . . . . . . 24

$\begin{array}{ll}\text { Homework 4: Geodesic Flow } & 27\end{array}$ 
5 Recurrence 29

5.1 Periodic Points . . . . . . . . . . . . . . . . . . . . . . . . . . . . . . . . 29

5.2 Billiards ... . . . . . . . . . . . . . . . . . 30

5.3 Poincaré Recurrence . . . . . . . . . . . . . . . . . . . . . . . . 32

III Local Forms 35

6 Preparation for the Local Theory 35

6.1 Isotopies and Vector Fields . . . . . . . . . . . . . . . . . . . . . . 35

6.2 Tubular Neighborhood Theorem . . . . . . . . . . . . . . . . 37

6.3 Homotopy Formula . . . . . . . . . . . . . . . . . . . . . . . . 39

Homework 5: Tubular Neighborhoods in $\mathbb{R}^{n} \quad 41$

7 Moser Theorems $\quad 42$

7.1 Notions of Equivalence for Symplectic Structures . . . . . . . . . . . 42

7.2 Moser Trick . . . . . . . . . . . . . . . . . . . . . . . . . 42

7.3 Moser Relative Theorem . . . . . . . . . . . . . . . . . . . 45

8 Darboux-Moser-Weinstein Theory 46

8.1 Darboux Theorem . . . . . . . . . . . . . . . . . . . . 46

8.2 Lagrangian Subspaces . . . . . . . . . . . . . . . . . . . . . 46

8.3 Weinstein Lagrangian Neighborhood Theorem . . . . . . . . . . . . 48

Homework 6: Oriented Surfaces $\quad 50$

9 Weinstein Tubular Neighborhood Theorem 51

9.1 Observation from Linear Algebra . . . . . . . . . . . . . . . . . . . 51

9.2 Tubular Neighborhoods . . . . . . . . . . . . . . . . . . 51

9.3 Application 1:

Tangent Space to the Group of Symplectomorphisms . . . . . . . . 53

9.4 Application 2:

Fixed Points of Symplectomorphisms . . . . . . . . . . . . . . . . . 55

IV Contact Manifolds 57

10 Contact Forms

10.1 Contact Structures . . . . . . . . . . . . . . . . . . 57

10.2 Examples. . . . . . . . . . . . . . . . . . . 58

10.3 First Properties . . . . . . . . . . . . . . . . . . . . . 59

Homework 7: Manifolds of Contact Elements $\quad 61$ 
11 Contact Dynamics 63

11.1 Reeb Vector Fields . . . . . . . . . . . . . . . . . . . . . . . 63

11.2 Symplectization . . . . . . . . . . . . . . . . . . . . 64

11.3 Conjectures of Seifert and Weinstein . . . . . . . . . . . . . . 65

V Compatible Almost Complex Structures 67

12 Almost Complex Structures 67

12.1 Three Geometries . . . . . . . . . . . . . . . . . . . . . . . . 67

12.2 Complex Structures on Vector Spaces . . . . . . . . . . . . . . . . 68

12.3 Compatible Structures . . . . . . . . . . . . . . . . . . . . . . . . . 70

Homework 8: Compatible Linear Structures $\quad 72$

13 Compatible Triples $\quad 74$

13.1 Compatibility . . . . . . . . . . . . . . . . . . 74

13.2 Triple of Structures . . . . . . . . . . . . . . . . . . . . . . . . . . . . . . . . . . . . . . . . . . . 75

13.3 First Consequences . . . . . . . . . . . . . . . . . . . 75

$\begin{array}{ll}\text { Homework 9: Contractibility } & 77\end{array}$

14 Dolbeault Theory 78

14.1 Splittings . . . . . . . . . . . . . . . . . . . . 78

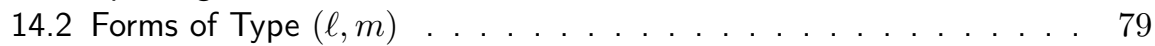

$14.3 \mathrm{~J}$-Holomorphic Functions . . . . . . . . . . . . . . . . . . . . . . . 80

14.4 Dolbeault Cohomology . . . . . . . . . . . . . . . . . 81

Homework 10: Integrability $\quad 82$

VI Kähler Manifolds 83

15 Complex Manifolds 83

15.1 Complex Charts . . . . . . . . . . . . . . . . . . . . . . . . 83

15.2 Forms on Complex Manifolds . . . . . . . . . . . . . . . . . . 85

15.3 Differentials . . . . . . . . . . . . . . . . . 86

Homework 11: Complex Projective Space $\quad 89$

16 Kähler Forms 90

16.1 Kähler Forms . . . . . . . . . . . . . . . . . . . . . . . . . . . 90

16.2 An Application . . . . . . . . . . . . . . . . . . . . . . . . . . . . . 92

16.3 Recipe to Obtain Kähler Forms . . . . . . . . . . . . . . . . . . . . 92

16.4 Local Canonical Form for Kähler Forms . . . . . . . . . . . . . . . 94

Homework 12: The Fubini-Study Structure 96 
17 Compact Kähler Manifolds 98

17.1 Hodge Theory . . . . . . . . . . . . . . . . . . . . . . . . . . . . . 98

17.2 Immediate Topological Consequences . . . . . . . . . . . . . . . . . 100

17.3 Compact Examples and Counterexamples . . . . . . . . . . . . . . 101

17.4 Main Kähler Manifolds . . . . . . . . . . . . . . . . . . . . . . . 103

$\begin{array}{ll}\text { VII Hamiltonian Mechanics } & 105\end{array}$

18 Hamiltonian Vector Fields 105

18.1 Hamiltonian and Symplectic Vector Fields . . . . . . . . . . . . . . 105

18.2 Classical Mechanics . . . . . . . . . . . . . . . . . . . . . 107

18.3 Brackets . . . . . . . . . . . . . . . . . . . . 108

18.4 Integrable Systems . . . . . . . . . . . . . . . . . . . . . 109

Homework 13: Simple Pendulum 112

19 Variational Principles 113

19.1 Equations of Motion . . . . . . . . . . . . . . . . . . . . . . . . . 113

19.2 Principle of Least Action . . . . . . . . . . . . . . . . . . . . . . . 114

19.3 Variational Problems . . . . . . . . . . . . . . . . . . . . . 114

19.4 Solving the Euler-Lagrange Equations . . . . . . . . . . . . . . . . 116

19.5 Minimizing Properties . . . . . . . . . . . . . . . . . . . . . . . . 117

Homework 14: Minimizing Geodesics $\quad 119$

20 Legendre Transform $\quad 121$

20.1 Strict Convexity . . . . . . . . . . . . . . . . . . . . . . . . . . . . 121

20.2 Legendre Transform . . . . . . . . . . . . . . . . . . . . . . . . . 121

20.3 Application to Variational Problems . . . . . . . . . . . . . . . . . 122

$\begin{array}{ll}\text { Homework 15: Legendre Transform } & 125\end{array}$

$\begin{array}{ll}\text { VIII Moment Maps } & 127\end{array}$

21 Actions 127

21.1 One-Parameter Groups of Diffeomorphisms . . . . . . . . . . . . . . 127

21.2 Lie Groups . . . . . . . . . . . . . . . . . . . . . . . . . . . . . . . 128

21.3 Smooth Actions . . . . . . . . . . . . . . . . . . . . . . . . . . . . 128

21.4 Symplectic and Hamiltonian Actions . . . . . . . . . . . . . . . . . 129

21.5 Adjoint and Coadjoint Representations . . . . . . . . . . . . . . . 130

Homework 16: Hermitian Matrices 132 
22 Hamiltonian Actions 133

22.1 Moment and Comoment Maps . . . . . . . . . . . . . . . . 133

22.2 Orbit Spaces . . . . . . . . . . . . . . . . . . . . . 135

22.3 Preview of Reduction . . . . . . . . . . . . . . . . . . 136

22.4 Classical Examples . . . . . . . . . . . . . . . . . . 137

Homework 17: Coadjoint Orbits 139

IX Symplectic Reduction $\quad 141$

23 The Marsden-Weinstein-Meyer Theorem 141

23.1 Statement . . . . . . . . . . . . . . . . . . . 141

23.2 Ingredients . . . . . . . . . . . . . . . . . . . . . . 142

23.3 Proof of the Marsden-Weinstein-Meyer Theorem . . . . . . . . . . . 145

24 Reduction 147

24.1 Noether Principle . . . . . . . . . . . . . . . . . . . . . . . . . . 147

24.2 Elementary Theory of Reduction . . . . . . . . . . . . . . . . . . 147

24.3 Reduction for Product Groups . . . . . . . . . . . . . . . . . . . . . 149

24.4 Reduction at Other Levels . . . . . . . . . . . . . . . . . . . . . . . 149

24.5 Orbifolds . . . . . . . . . . . . . . . . . . . . 150

Homework 18: Spherical Pendulum 152

X Moment Maps Revisited 155

25 Moment Map in Gauge Theory 155

25.1 Connections on a Principal Bundle . . . . . . . . . . . . . . . . . . 155

25.2 Connection and Curvature Forms . . . . . . . . . . . . . . . . . . . 156

25.3 Symplectic Structure on the Space of Connections . . . . . . . . . . 158

25.4 Action of the Gauge Group . . . . . . . . . . . . . . . . . . . . . . 158

25.5 Case of Circle Bundles . . . . . . . . . . . . . . . . . . . . . . 159

Homework 19: Examples of Moment Maps 162

26 Existence and Uniqueness of Moment Maps 164

26.1 Lie Algebras of Vector Fields . . . . . . . . . . . . . . . . . . . . . 164

26.2 Lie Algebra Cohomology . . . . . . . . . . . . . . . . . . . . 165

26.3 Existence of Moment Maps . . . . . . . . . . . . . . . . . . . . . . 166

26.4 Uniqueness of Moment Maps . . . . . . . . . . . . . . . . . 167

Homework 20: Examples of Reduction 168 
27 Convexity 169

27.1 Convexity Theorem . . . . . . . . . . . . . . . . . . . . 169

27.2 Effective Actions . . . . . . . . . . . . . . . . . . 170

27.3 Examples . . . . . . . . . . . . . . . . . . . . . . 172

Homework 21: Connectedness 174

XI Symplectic Toric Manifolds 177

28 Classification of Symplectic Toric Manifolds 177

28.1 Delzant Polytopes . . . . . . . . . . . . . . . . . 177

28.2 Delzant Theorem . . . . . . . . . . . . . . . . . . . . . 179

28.3 Sketch of Delzant Construction . . . . . . . . . . . . . . . . 180

29 Delzant Construction 183

29.1 Algebraic Set-Up. . . . . . . . . . . . . . . . . . 183

29.2 The Zero-Level . . . . . . . . . . . . . . . . . . . . . 183

29.3 Conclusion of the Delzant Construction . . . . . . . . . . . 185

29.4 Idea Behind the Delzant Construction . . . . . . . . . . . 186

Homework 22: Delzant Theorem 189

30 Duistermaat-Heckman Theorems 191

30.1 Duistermaat-Heckman Polynomial . . . . . . . . . . . . . . . 191

30.2 Local Form for Reduced Spaces . . . . . . . . . . . . . . . . . . . 192

30.3 Variation of the Symplectic Volume . . . . . . . . . . . . 195

$\begin{array}{ll}\text { Homework 23: } S^{1} \text {-Equivariant Cohomology } & 197\end{array}$

$\begin{array}{ll}\text { References } & 199\end{array}$

$\begin{array}{ll}\text { Index } & 207\end{array}$ 


\section{Introduction}

The goal of these notes is to provide a fast introduction to symplectic geometry.

A symplectic form is a closed nondegenerate 2-form. A symplectic manifold is a manifold equipped with a symplectic form. Symplectic geometry is the geometry of symplectic manifolds. Symplectic manifolds are necessarily even-dimensional and orientable, since nondegeneracy says that the top exterior power of a symplectic form is a volume form. The closedness condition is a natural differential equation, which forces all symplectic manifolds to being locally indistinguishable. (These assertions will be explained in Lecture 1 and Homework 2.)

The list of questions on symplectic forms begins with those of existence and uniqueness on a given manifold. For specific symplectic manifolds, one would like to understand the geometry and the topology of special submanifolds, the dynamics of certain vector fields or systems of differential equations, the symmetries and extra structure, etc.

Two centuries ago, symplectic geometry provided a language for classical mechanics. Through its recent huge development, it conquered an independent and rich territory, as a central branch of differential geometry and topology. To mention just a few key landmarks, one may say that symplectic geometry began to take its modern shape with the formulation of the Arnold conjectures in the 60's and with the foundational work of Weinstein in the 70's. A paper of Gromov [49] in the 80's gave the subject a whole new set of tools: pseudo-holomorphic curves. Gromov also first showed that important results from complex Kähler geometry remain true in the more general symplectic category, and this direction was continued rather dramatically in the 90's in the work of Donaldson on the topology of symplectic manifolds and their symplectic submanifolds, and in the work of Taubes in the context of the Seiberg-Witten invariants. Symplectic geometry is significantly stimulated by important interactions with global analysis, mathematical physics, low-dimensional topology, dynamical systems, algebraic geometry, integrable systems, microlocal analysis, partial differential equations, representation theory, quantization, equivariant cohomology, geometric combinatorics, etc.

As a curiosity, note that two centuries ago the name symplectic geometry did not exist. If you consult a major English dictionary, you are likely to find that symplectic is the name for a bone in a fish's head. However, as clarified in [105], the word symplectic in mathematics was coined by Weyl [110, p.165] who substituted the Latin root in complex by the corresponding Greek root, in order to label the symplectic group. Weyl thus avoided that this group connote the complex numbers, and also spared us from much confusion that would have arisen, had the name remained the former one in honor of Abel: abelian linear group.

This text is essentially the set of notes of a 15-week course on symplectic geometry with 2 hour-and-a-half lectures per week. The course targeted second-year graduate students in mathematics, though the audience was more diverse, including advanced undergraduates, post-docs and graduate students from other departments. The present text should hence still be appropriate for a second-year graduate course or for an independent study project. 
There are scattered short exercises throughout the text. At the end of most lectures, some longer guided problems, called homework, were designed to complement the exposition or extend the reader's understanding.

Geometry of manifolds was the basic prerequisite for the original course, so the same holds now for the notes. In particular, some familiarity with de Rham theory and classical Lie groups is expected.

As for conventions: unless otherwise indicated, all vector spaces are real and finite-dimensional, all maps are smooth (i.e., $C^{\infty}$ ) and all manifolds are smooth, Hausdorff and second countable.

Here is a brief summary of the contents of this book. Parts I-III explain classical topics, including cotangent bundles, symplectomorphisms, lagrangian submanifolds and local forms. Parts IV-VI concentrate on important related areas, such as contact geometry and Kähler geometry. Classical hamiltonian theory enters in Parts VII-VIII, starting the second half of this book, which is devoted to a selection of themes from hamiltonian dynamical systems and symmetry. Parts IX-XI discuss the moment map whose preponderance has been growing steadily for the past twenty years.

There are by now excellent references on symplectic geometry, a subset of which is in the bibliography. However, the most efficient introduction to a subject is often a short elementary treatment, and these notes attempt to serve that purpose. The author hopes that these notes provide a taste of areas of current research, and will prepare the reader to explore recent papers and extensive books in symplectic geometry, where the pace is much faster. 


\section{Part I}

\section{Symplectic Manifolds}

A symplectic form is a 2-form satisfying an algebraic condition - nondegeneracy and an analytical condition - closedness. In Lectures 1 and 2 we define symplectic forms, describe some of their basic properties, introduce the first examples, namely even-dimensional euclidean spaces and cotangent bundles.

\section{Symplectic Forms}

\subsection{Skew-Symmetric Bilinear Maps}

Let $V$ be an $m$-dimensional vector space over $\mathbb{R}$, and let $\Omega: V \times V \rightarrow \mathbb{R}$ be a bilinear map. The map $\Omega$ is skew-symmetric if $\Omega(u, v)=-\Omega(v, u)$, for all $u, v \in V$.

Theorem 1.1 (Standard Form for Skew-symmetric Bilinear Maps)

Let $\Omega$ be a skew-symmetric bilinear map on $V$. Then there is a basis $u_{1}, \ldots, u_{k}, e_{1}, \ldots, e_{n}, f_{1}, \ldots, f_{n}$ of $V$ such that

$$
\begin{array}{ll}
\Omega\left(u_{i}, v\right)=0, & \text { for all } i \text { and all } v \in V, \\
\Omega\left(e_{i}, e_{j}\right)=0=\Omega\left(f_{i}, f_{j}\right), & \text { for all } i, j, \text { and } \\
\Omega\left(e_{i}, f_{j}\right)=\delta_{i j}, & \text { for all } i, j .
\end{array}
$$

\section{Remarks.}

1. The basis in Theorem 1.1 is not unique, though it is traditionally also called a "canonical" basis.

2. In matrix notation with respect to such basis, we have

$$
\Omega(u, v)=[-u-]\left[\begin{array}{ccc}
0 & 0 & 0 \\
0 & 0 & \text { Id } \\
0 & - \text { Id } & 0
\end{array}\right]\left[\begin{array}{l}
\mid \\
v \\
\mid
\end{array}\right] .
$$

Proof. This induction proof is a skew-symmetric version of the Gram-Schmidt process.

Let $U:=\{u \in V \mid \Omega(u, v)=0$ for all $v \in V\}$. Choose a basis $u_{1}, \ldots, u_{k}$ of $U$, and choose a complementary space $W$ to $U$ in $V$,

$$
V=U \oplus W .
$$


Take any nonzero $e_{1} \in W$. Then there is $f_{1} \in W$ such that $\Omega\left(e_{1}, f_{1}\right) \neq 0$. Assume that $\Omega\left(e_{1}, f_{1}\right)=1$. Let

$$
\begin{aligned}
W_{1} & =\text { span of } e_{1}, f_{1} \\
W_{1}^{\Omega} & =\left\{w \in W \mid \Omega(w, v)=0 \text { for all } v \in W_{1}\right\} .
\end{aligned}
$$

Claim. $W_{1} \cap W_{1}^{\Omega}=\{0\}$.

Suppose that $v=a e_{1}+b f_{1} \in W_{1} \cap W_{1}^{\Omega}$.

$$
\left.\begin{array}{l}
0=\Omega\left(v, e_{1}\right)=-b \\
0=\Omega\left(v, f_{1}\right)=a
\end{array}\right\} \quad \Longrightarrow \quad v=0
$$

Claim. $W=W_{1} \oplus W_{1}^{\Omega}$.

Suppose that $v \in W$ has $\Omega\left(v, e_{1}\right)=c$ and $\Omega\left(v, f_{1}\right)=d$. Then

$$
v=\underbrace{\left(-c f_{1}+d e_{1}\right)}_{\in W_{1}}+\underbrace{\left(v+c f_{1}-d e_{1}\right)}_{\in W_{1}^{\Omega}} .
$$

Go on: let $e_{2} \in W_{1}^{\Omega}, e_{2} \neq 0$. There is $f_{2} \in W_{1}^{\Omega}$ such that $\Omega\left(e_{2}, f_{2}\right) \neq 0$. Assume that $\Omega\left(e_{2}, f_{2}\right)=1$. Let $W_{2}=$ span of $e_{2}, f_{2}$. Etc.

This process eventually stops because $\operatorname{dim} V<\infty$. We hence obtain

$$
V=U \oplus W_{1} \oplus W_{2} \oplus \ldots \oplus W_{n}
$$

where all summands are orthogonal with respect to $\Omega$, and where $W_{i}$ has basis $e_{i}, f_{i}$ with $\Omega\left(e_{i}, f_{i}\right)=1$.

The dimension of the subspace $U=\{u \in V \mid \Omega(u, v)=0$, for all $v \in V\}$ does not depend on the choice of basis.

$\Longrightarrow \quad k:=\operatorname{dim} U$ is an invariant of $(V, \Omega)$.

Since $k+2 n=m=\operatorname{dim} V$,

$\Longrightarrow \quad n$ is an invariant of $(V, \Omega) ; 2 n$ is called the rank of $\Omega$.

\subsection{Symplectic Vector Spaces}

Let $V$ be an $m$-dimensional vector space over $\mathbb{R}$, and let $\Omega: V \times V \rightarrow \mathbb{R}$ be a bilinear map.

Definition 1.2 The map $\widetilde{\Omega}: V \rightarrow V^{*}$ is the linear map defined by $\widetilde{\Omega}(v)(u)=$ $\Omega(v, u)$.

The kernel of $\widetilde{\Omega}$ is the subspace $U$ above.

Definition 1.3 A skew-symmetric bilinear map $\Omega$ is symplectic (or nondegenerate) if $\widetilde{\Omega}$ is bijective, i.e., $U=\{0\}$. The map $\Omega$ is then called a linear symplectic structure on $V$, and $(V, \Omega)$ is called a symplectic vector space. 
The following are immediate properties of a linear symplectic structure $\Omega$ :

- Duality: the map $\widetilde{\Omega}: V \stackrel{\simeq}{\leftrightarrows} V^{*}$ is a bijection.

- By the standard form theorem, $k=\operatorname{dim} U=0$, so $\operatorname{dim} V=2 n$ is even.

- By Theorem 1.1, a symplectic vector space $(V, \Omega)$ has a basis $e_{1}, \ldots, e_{n}, f_{1}, \ldots, f_{n}$ satisfying

$$
\Omega\left(e_{i}, f_{j}\right)=\delta_{i j} \quad \text { and } \quad \Omega\left(e_{i}, e_{j}\right)=0=\Omega\left(f_{i}, f_{j}\right) .
$$

Such a basis is called a symplectic basis of $(V, \Omega)$. We have

$$
\Omega(u, v)=[-u-]\left[\begin{array}{cc}
0 & \mathrm{Id} \\
-\mathrm{Id} & 0
\end{array}\right]\left[\begin{array}{c}
\mid \\
v \\
\mid
\end{array}\right]
$$

where the symbol $\left[\begin{array}{l}1 \\ v \\ 1\end{array}\right]$ represents the column of coordinates of the vector $v$ with respect to a symplectic basis $e_{1}, \ldots, e_{n}, f_{1}, \ldots, f_{n}$ whereas $[-v-]$ represents its transpose line.

Not all subspaces $W$ of a symplectic vector space $(V, \Omega)$ look the same:

- A subspace $W$ is called symplectic if $\left.\Omega\right|_{W}$ is nondegenerate. For instance, the span of $e_{1}, f_{1}$ is symplectic.

- A subspace $W$ is called isotropic if $\left.\Omega\right|_{W} \equiv 0$. For instance, the span of $e_{1}, e_{2}$ is isotropic.

Homework 1 describes subspaces $W$ of $(V, \Omega)$ in terms of the relation between $W$ and $W^{\Omega}$.

The prototype of a symplectic vector space is $\left(\mathbb{R}^{2 n}, \Omega_{0}\right)$ with $\Omega_{0}$ such that the basis

$$
\begin{array}{lll}
e_{1}=(1,0, \ldots, 0), & \ldots, & e_{n}=(0, \ldots, 0, \overbrace{1}^{n}, 0, \ldots, 0), \\
f_{1}=(0, \ldots, 0, \underbrace{1}_{n+1}, 0, \ldots, 0), & \ldots, & f_{n}=(0, \ldots, 0,1)
\end{array}
$$

is a symplectic basis. The map $\Omega_{0}$ on other vectors is determined by its values on a basis and bilinearity.

Definition 1.4 A symplectomorphism $\varphi$ between symplectic vector spaces $(V, \Omega)$ and $\left(V^{\prime}, \Omega^{\prime}\right)$ is a linear isomorphism $\varphi: V \stackrel{\simeq}{\rightarrow} V^{\prime}$ such that $\varphi^{*} \Omega^{\prime}=\Omega$. (By definition, $\left(\varphi^{*} \Omega^{\prime}\right)(u, v)=\Omega^{\prime}(\varphi(u), \varphi(v))$.) If a symplectomorphism exists, $(V, \Omega)$ and $\left(V^{\prime}, \Omega^{\prime}\right)$ are said to be symplectomorphic. 
The relation of being symplectomorphic is clearly an equivalence relation in the set of all even-dimensional vector spaces. Furthermore, by Theorem 1.1, every $2 n$ dimensional symplectic vector space $(V, \Omega)$ is symplectomorphic to the prototype $\left(\mathbb{R}^{2 n}, \Omega_{0}\right)$; a choice of a symplectic basis for $(V, \Omega)$ yields a symplectomorphism to $\left(\mathbb{R}^{2 n}, \Omega_{0}\right)$. Hence, nonnegative even integers classify equivalence classes for the relation of being symplectomorphic.

\subsection{Symplectic Manifolds}

Let $\omega$ be a de Rham 2-form on a manifold $M$, that is, for each $p \in M$, the map $\omega_{p}: T_{p} M \times T_{p} M \rightarrow \mathbb{R}$ is skew-symmetric bilinear on the tangent space to $M$ at $p$, and $\omega_{p}$ varies smoothly in $p$. We say that $\omega$ is closed if it satisfies the differential equation $d \omega=0$, where $d$ is the de Rham differential (i.e., exterior derivative).

Definition 1.5 The 2-form $\omega$ is symplectic if $\omega$ is closed and $\omega_{p}$ is symplectic for all $p \in M$.

If $\omega$ is symplectic, then $\operatorname{dim} T_{p} M=\operatorname{dim} M$ must be even.

Definition 1.6 A symplectic manifold is a pair $(M, \omega)$ where $M$ is a manifold and $\omega$ is a symplectic form.

Example. Let $M=\mathbb{R}^{2 n}$ with linear coordinates $x_{1}, \ldots, x_{n}, y_{1}, \ldots, y_{n}$. The form

$$
\omega_{0}=\sum_{i=1}^{n} d x_{i} \wedge d y_{i}
$$

is symplectic as can be easily checked, and the set

$$
\left\{\left(\frac{\partial}{\partial x_{1}}\right)_{p}, \ldots,\left(\frac{\partial}{\partial x_{n}}\right)_{p},\left(\frac{\partial}{\partial y_{1}}\right)_{p}, \ldots,\left(\frac{\partial}{\partial y_{n}}\right)_{p}\right\}
$$

is a symplectic basis of $T_{p} M$.

Example. Let $M=\mathbb{C}^{n}$ with linear coordinates $z_{1}, \ldots, z_{n}$. The form

$$
\omega_{0}=\frac{i}{2} \sum_{k=1}^{n} d z_{k} \wedge d \bar{z}_{k}
$$

is symplectic. In fact, this form equals that of the previous example under the identification $\mathbb{C}^{n} \simeq \mathbb{R}^{2 n}, z_{k}=x_{k}+i y_{k}$.

Example. Let $M=S^{2}$ regarded as the set of unit vectors in $\mathbb{R}^{3}$. Tangent vectors to $S^{2}$ at $p$ may then be identified with vectors orthogonal to $p$. The standard symplectic form on $S^{2}$ is induced by the inner and exterior products:

$$
\omega_{p}(u, v):=\langle p, u \times v\rangle, \quad \text { for } u, v \in T_{p} S^{2}=\{p\}^{\perp} .
$$

This form is closed because it is of top degree; it is nondegenerate because $\langle p, u \times$ $v\rangle \neq 0$ when $u \neq 0$ and we take, for instance, $v=u \times p$. 


\subsection{Symplectomorphisms}

Definition 1.7 Let $\left(M_{1}, \omega_{1}\right)$ and $\left(M_{2}, \omega_{2}\right)$ be $2 n$-dimensional symplectic manifolds, and let $\varphi: M_{1} \rightarrow M_{2}$ be a diffeomorphism. Then $\varphi$ is a symplectomorphism if $\varphi^{*} \omega_{2}=\omega_{1}{ }^{1}$

We would like to classify symplectic manifolds up to symplectomorphism. The Darboux theorem (proved in Lecture 8 and stated below) takes care of this classification locally: the dimension is the only local invariant of symplectic manifolds up to symplectomorphisms. Just as any $n$-dimensional manifold looks locally like $\mathbb{R}^{n}$, any $2 n$-dimensional symplectic manifold looks locally like $\left(\mathbb{R}^{2 n}, \omega_{0}\right)$. More precisely, any symplectic manifold $\left(M^{2 n}, \omega\right)$ is locally symplectomorphic to $\left(\mathbb{R}^{2 n}, \omega_{0}\right)$.

Theorem 8.1 (Darboux) Let $(M, \omega)$ be a $2 n$-dimensional symplectic manifold, and let $p$ be any point in $M$.

Then there is a coordinate chart $\left(\mathcal{U}, x_{1}, \ldots, x_{n}, y_{1}, \ldots, y_{n}\right)$ centered at $p$ such that on $\mathcal{U}$

$$
\omega=\sum_{i=1}^{n} d x_{i} \wedge d y_{i}
$$

A chart $\left(\mathcal{U}, x_{1}, \ldots, x_{n}, y_{1}, \ldots, y_{n}\right)$ as in Theorem 8.1 is called a Darboux chart. By Theorem 8.1, the prototype of a local piece of a $2 n$-dimensional symplectic manifold is $M=\mathbb{R}^{2 n}$, with linear coordinates $\left(x_{1}, \ldots, x_{n}, y_{1}, \ldots, y_{n}\right)$, and with symplectic form

$$
\omega_{0}=\sum_{i=1}^{n} d x_{i} \wedge d y_{i}
$$

\footnotetext{
${ }^{1}$ Recall that, by definition of pullback, at tangent vectors $u, v \in T_{p} M_{1}$, we have $\left(\varphi^{*} \omega_{2}\right)_{p}(u, v)=\left(\omega_{2}\right)_{\varphi(p)}\left(d \varphi_{p}(u), d \varphi_{p}(v)\right)$.
} 


\section{Homework 1: Symplectic Linear Algebra}

Given a linear subspace $Y$ of a symplectic vector space $(V, \Omega)$, its symplectic orthogonal $Y^{\Omega}$ is the linear subspace defined by

$$
Y^{\Omega}:=\{v \in V \mid \Omega(v, u)=0 \text { for all } u \in Y\} .
$$

1. Show that $\operatorname{dim} Y+\operatorname{dim} Y^{\Omega}=\operatorname{dim} V$.

Hint: What is the kernel and image of the map

$$
\begin{aligned}
V & \longrightarrow Y^{*}=\operatorname{Hom}(Y, \mathbb{R}) \quad ? \\
v & \left.\longmapsto \Omega(v, \cdot)\right|_{Y}
\end{aligned}
$$

2. Show that $\left(Y^{\Omega}\right)^{\Omega}=Y$.

3. Show that, if $Y$ and $W$ are subspaces, then

$$
Y \subseteq W \Longleftrightarrow W^{\Omega} \subseteq Y^{\Omega} .
$$

4. Show that:

$Y$ is symplectic (i.e., $\left.\Omega\right|_{Y \times Y}$ is nondegenerate) $\Longleftrightarrow Y \cap Y^{\Omega}=\{0\} \Longleftrightarrow$ $V=Y \oplus Y^{\Omega}$.

5. We call $Y$ isotropic when $Y \subseteq Y^{\Omega}$ (i.e., $\left.\Omega\right|_{Y \times Y} \equiv 0$ ).

Show that, if $Y$ is isotropic, then $\operatorname{dim} Y \leq \frac{1}{2} \operatorname{dim} V$.

6. We call $Y$ coisotropic when $Y^{\Omega} \subseteq Y$.

Check that every codimension 1 subspace $Y$ is coisotropic.

7. An isotropic subspace $Y$ of $(V, \Omega)$ is called lagrangian when $\operatorname{dim} Y=\frac{1}{2} \operatorname{dim} V$. Check that:

$$
Y \text { is lagrangian } \Longleftrightarrow Y \text { is isotropic and coisotropic } \Longleftrightarrow Y=Y^{\Omega} .
$$

8. Show that, if $Y$ is a lagrangian subspace of $(V, \Omega)$, then any basis $e_{1}, \ldots, e_{n}$ of $Y$ can be extended to a symplectic basis $e_{1}, \ldots, e_{n}, f_{1}, \ldots, f_{n}$ of $(V, \Omega)$.

Hint: Choose $f_{1}$ in $W^{\Omega}$, where $W$ is the linear span of $\left\{e_{2}, \ldots, e_{n}\right\}$.

9. Show that, if $Y$ is a lagrangian subspace, $(V, \Omega)$ is symplectomorphic to the space $\left(Y \oplus Y^{*}, \Omega_{0}\right)$, where $\Omega_{0}$ is determined by the formula

$$
\Omega_{0}(u \oplus \alpha, v \oplus \beta)=\beta(u)-\alpha(v) .
$$

In fact, for any vector space $E$, the direct sum $V=E \oplus E^{*}$ has a canonical symplectic structure determined by the formula above. If $e_{1}, \ldots, e_{n}$ is a basis of $E$, and $f_{1}, \ldots, f_{n}$ is the dual basis, then $e_{1} \oplus 0, \ldots, e_{n} \oplus 0,0 \oplus f_{1}, \ldots, 0 \oplus f_{n}$ is a symplectic basis for $V$. 


\section{Symplectic Form on the Cotangent Bundle}

\subsection{Cotangent Bundle}

Let $X$ be any $n$-dimensional manifold and $M=T^{*} X$ its cotangent bundle. If the manifold structure on $X$ is described by coordinate charts $\left(\mathcal{U}, x_{1}, \ldots, x_{n}\right)$ with $x_{i}: \mathcal{U} \rightarrow \mathbb{R}$, then at any $x \in \mathcal{U}$, the differentials $\left(d x_{1}\right)_{x}, \ldots\left(d x_{n}\right)_{x}$ form a basis of $T_{x}^{*} X$. Namely, if $\xi \in T_{x}^{*} X$, then $\xi=\sum_{i=1}^{n} \xi_{i}\left(d x_{i}\right)_{x}$ for some real coefficients $\xi_{1}, \ldots, \xi_{n}$. This induces a map

$$
\begin{aligned}
T^{*} \mathcal{U} & \longrightarrow \mathbb{R}^{2 n} \\
(x, \xi) & \longmapsto\left(x_{1}, \ldots, x_{n}, \xi_{1}, \ldots, \xi_{n}\right) .
\end{aligned}
$$

The chart $\left(T^{*} \mathcal{U}, x_{1}, \ldots, x_{n}, \xi_{1}, \ldots, \xi_{n}\right)$ is a coordinate chart for $T^{*} X$; the coordinates $x_{1}, \ldots, x_{n}, \xi_{1}, \ldots, \xi_{n}$ are the cotangent coordinates associated to the coordinates $x_{1}, \ldots, x_{n}$ on $\mathcal{U}$. The transition functions on the overlaps are smooth: given two charts $\left(\mathcal{U}, x_{1}, \ldots, x_{n}\right),\left(\mathcal{U}^{\prime}, x_{1}^{\prime}, \ldots, x_{n}^{\prime}\right)$, and $x \in \mathcal{U} \cap \mathcal{U}^{\prime}$, if $\xi \in T_{x}^{*} X$, then

$$
\xi=\sum_{i=1}^{n} \xi_{i}\left(d x_{i}\right)_{x}=\sum_{i, j} \xi_{i}\left(\frac{\partial x_{i}}{\partial x_{j}^{\prime}}\right)\left(d x_{j}^{\prime}\right)_{x}=\sum_{j=1}^{n} \xi_{j}^{\prime}\left(d x_{j}^{\prime}\right)_{x}
$$

where $\xi_{j}^{\prime}=\sum_{i} \xi_{i}\left(\frac{\partial x_{i}}{\partial x_{j}^{\prime}}\right)$ is smooth. Hence, $T^{*} X$ is a $2 n$-dimensional manifold.

We will now construct a major class of examples of symplectic forms. The canonical forms on cotangent bundles are relevant for several branches, including analysis of differential operators, dynamical systems and classical mechanics.

\subsection{Tautological and Canonical Forms in Coordinates}

Let $\left(\mathcal{U}, x_{1}, \ldots, x_{n}\right)$ be a coordinate chart for $X$, with associated cotangent coordinates $\left(T^{*} \mathcal{U}, x_{1}, \ldots, x_{n}, \xi_{1}, \ldots, \xi_{n}\right)$. Define a 2 -form $\omega$ on $T^{*} \mathcal{U}$ by

$$
\omega=\sum_{i=1}^{n} d x_{i} \wedge d \xi_{i}
$$

In order to check that this definition is coordinate-independent, consider the 1-form on $T^{*} \mathcal{U}$

$$
\alpha=\sum_{i=1}^{n} \xi_{i} d x_{i}
$$

Clearly, $\omega=-d \alpha$.

Claim. The form $\alpha$ is intrinsically defined (and hence the form $\omega$ is also intrinsically defined) . 
Proof. Let $\left(\mathcal{U}, x_{1}, \ldots, x_{n}, \xi_{1}, \ldots, \xi_{n}\right)$ and $\left(\mathcal{U}^{\prime}, x_{1}^{\prime}, \ldots, x_{n}^{\prime}, \xi_{1}^{\prime}, \ldots, \xi_{n}^{\prime}\right)$ be two cotangent coordinate charts. On $\mathcal{U} \cap \mathcal{U}^{\prime}$, the two sets of coordinates are related by $\xi_{j}^{\prime}=\sum_{i} \xi_{i}\left(\frac{\partial x_{i}}{\partial x_{j}^{\prime}}\right)$. Since $d x_{j}^{\prime}=\sum_{i}\left(\frac{\partial x_{j}^{\prime}}{\partial x_{i}}\right) d x_{i}$, we have

$$
\alpha=\sum_{i} \xi_{i} d x_{i}=\sum_{j} \xi_{j}^{\prime} d x_{j}^{\prime}=\alpha^{\prime}
$$

The 1-form $\alpha$ is the tautological form or Liouville 1-form and the 2-form $\omega$ is the canonical symplectic form. The following section provides an alternative proof of the intrinsic character of these forms.

\subsection{Coordinate-Free Definitions}

Let

$$
\begin{array}{cc}
M=T^{*} X & p=(x, \xi) \quad \xi \in T_{x}^{*} X \\
\downarrow \pi & \downarrow \\
X & x
\end{array}
$$

be the natural projection. The tautological 1-form $\alpha$ may be defined pointwise by

$$
\alpha_{p}=\left(d \pi_{p}\right)^{*} \xi \quad \in T_{p}^{*} M,
$$

where $\left(d \pi_{p}\right)^{*}$ is the transpose of $d \pi_{p}$, that is, $\left(d \pi_{p}\right)^{*} \xi=\xi \circ d \pi_{p}$ :

$$
\begin{array}{ccc}
p=(x, \xi) & T_{p} M & T_{p}^{*} M \\
\downarrow \pi & \downarrow d \pi_{p} & \uparrow\left(d \pi_{p}\right)^{*} \\
x & T_{x} X & T_{x}^{*} X
\end{array}
$$

Equivalently,

$$
\alpha_{p}(v)=\xi\left(\left(d \pi_{p}\right) v\right), \quad \text { for } v \in T_{p} M
$$

Exercise. Let $\left(\mathcal{U}, x_{1}, \ldots, x_{n}\right)$ be a chart on $X$ with associated cotangent coordinates $x_{1}, \ldots, x_{n}, \xi_{1}, \ldots, \xi_{n}$. Show that on $T^{*} \mathcal{U}, \alpha=\sum_{i=1}^{n} \xi_{i} d x_{i}$.

The canonical symplectic 2 -form $\omega$ on $T^{*} X$ is defined as

$$
\omega=-d \alpha
$$

Locally, $\omega=\sum_{i=1}^{n} d x_{i} \wedge d \xi_{i}$.

Exercise. Show that the tautological 1 -form $\alpha$ is uniquely characterized by the property that, for every 1-form $\mu: X \rightarrow T^{*} X, \mu^{*} \alpha=\mu$. (See Lecture 3.) 


\subsection{Naturality of the Tautological and Canonical Forms}

Let $X_{1}$ and $X_{2}$ be $n$-dimensional manifolds with cotangent bundles $M_{1}=T^{*} X_{1}$ and $M_{2}=T^{*} X_{2}$, and tautological 1-forms $\alpha_{1}$ and $\alpha_{2}$. Suppose that $f: X_{1} \rightarrow X_{2}$ is a diffeomorphism. Then there is a natural diffeomorphism

$$
f_{\sharp}: M_{1} \rightarrow M_{2}
$$

which lifts $f$; namely, if $p_{1}=\left(x_{1}, \xi_{1}\right) \in M_{1}$ for $x_{1} \in X_{1}$ and $\xi_{1} \in T_{x_{1}}^{*} X_{1}$, then we define

$$
f_{\sharp}\left(p_{1}\right)=p_{2}=\left(x_{2}, \xi_{2}\right), \quad \text { with }\left\{\begin{array}{l}
x_{2}=f\left(x_{1}\right) \in X_{2} \text { and } \\
\xi_{1}=\left(d f_{x_{1}}\right)^{*} \xi_{2},
\end{array}\right.
$$

where $\left(d f_{x_{1}}\right)^{*}: T_{x_{2}}^{*} X_{2} \stackrel{\simeq}{\rightrightarrows} T_{x_{1}}^{*} X_{1}$, so $\left.f_{\sharp}\right|_{T_{x_{1}}^{*}}$ is the inverse map of $\left(d f_{x_{1}}\right)^{*}$.

Exercise. Check that $f_{\sharp}$ is a diffeomorphism. Here are some hints:

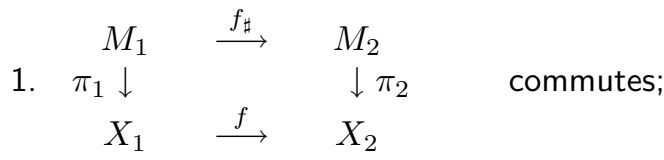

2. $f_{\sharp}: M_{1} \rightarrow M_{2}$ is bijective;

3. $f_{\sharp}$ and $f_{\sharp}^{-1}$ are smooth.

Proposition 2.1 The lift $f_{\sharp}$ of a diffeomorphism $f: X_{1} \rightarrow X_{2}$ pulls the tautological form on $T^{*} X_{2}$ back to the tautological form on $T^{*} X_{1}$, i.e.,

$$
\left(f_{\sharp}\right)^{*} \alpha_{2}=\alpha_{1} .
$$

Proof. At $p_{1}=\left(x_{1}, \xi_{1}\right) \in M_{1}$, this identity says

$$
\left(d f_{\sharp}\right)_{p_{1}}^{*}\left(\alpha_{2}\right)_{p_{2}}=\left(\alpha_{1}\right)_{p_{1}}
$$

where $p_{2}=f_{\sharp}\left(p_{1}\right)$.

Using the following facts,

- Definition of $f_{\sharp}$ :

$p_{2}=f_{\sharp}\left(p_{1}\right) \Longleftrightarrow p_{2}=\left(x_{2}, \xi_{2}\right)$ where $x_{2}=f\left(x_{1}\right)$ and $\left(d f_{x_{1}}\right)^{*} \xi_{2}=\xi_{1}$.

- Definition of tautological 1-form:

$\left(\alpha_{1}\right)_{p_{1}}=\left(d \pi_{1}\right)_{p_{1}}^{*} \xi_{1} \quad$ and $\quad\left(\alpha_{2}\right)_{p_{2}}=\left(d \pi_{2}\right)_{p_{2}}^{*} \xi_{2}$.

- The diagram

$\begin{array}{cccc}M_{1} & \stackrel{f_{\sharp}}{\longrightarrow} & M_{2} & \\ \pi_{1} \downarrow & & \downarrow \pi_{2} & \text { commutes. } \\ X_{1} & \stackrel{f}{\longrightarrow} & X_{2} & \end{array}$


the proof of $(\star)$ is:

$$
\begin{aligned}
& \left(d f_{\sharp}\right)_{p_{1}}^{*}\left(\alpha_{2}\right)_{p_{2}}=\left(d f_{\sharp}\right)_{p_{1}}^{*}\left(d \pi_{2}\right)_{p_{2}}^{*} \xi_{2}=\left(d\left(\pi_{2} \circ f_{\sharp}\right)\right)_{p_{1}}^{*} \xi_{2} \\
& =\left(d\left(f \circ \pi_{1}\right)\right)_{p_{1}}^{*} \xi_{2}=\left(d \pi_{1}\right)_{p_{1}}^{*}(d f)_{x_{1}}^{*} \xi_{2} \\
& =\left(d \pi_{1}\right)_{p_{1}}^{*} \xi_{1}=\left(\alpha_{1}\right)_{p_{1}} \text {. }
\end{aligned}
$$

Corollary 2.2 The lift $f_{\sharp}$ of a diffeomorphism $f: X_{1} \rightarrow X_{2}$ is a symplectomorphism, i.e.,

$$
\left(f_{\sharp}\right)^{*} \omega_{2}=\omega_{1},
$$

where $\omega_{1}, \omega_{2}$ are the canonical symplectic forms.

In summary, a diffeomorphism of manifolds induces a canonical symplectomorphism of cotangent bundles:

$$
\begin{array}{cccc}
f_{\sharp}: & T^{*} X_{1} & \longrightarrow & T^{*} X_{2} \\
f: & X_{1} & \longrightarrow & X_{2}
\end{array}
$$

Example. Let $X_{1}=X_{2}=S^{1}$. Then $T^{*} S^{1}$ is an infinite cylinder $S^{1} \times \mathbb{R}$. The canonical 2-form $\omega$ is the area form $\omega=d \theta \wedge d \xi$. If $f: S^{1} \rightarrow S^{1}$ is any diffeomorphism, then $f_{\sharp}: S^{1} \times \mathbb{R} \rightarrow S^{1} \times \mathbb{R}$ is a symplectomorphism, i.e., is an area-preserving diffeomorphism of the cylinder.

If $f: X_{1} \rightarrow X_{2}$ and $g: X_{2} \rightarrow X_{3}$ are diffeomorphisms, then $(g \circ f)_{\sharp}=g_{\sharp} \circ f_{\sharp}$. In terms of the group $\operatorname{Diff}(X)$ of diffeomorphisms of $X$ and the group $\operatorname{Sympl}(M, \omega)$ of symplectomorphisms of $(M, \omega)$, we say that the map

$$
\begin{aligned}
\operatorname{Diff}(X) & \longrightarrow \operatorname{Sympl}(M, \omega) \\
f & \longmapsto f_{\sharp}
\end{aligned}
$$

is a group homomorphism. This map is clearly injective. Is it surjective? Do all symplectomorphisms $T^{*} X \rightarrow T^{*} X$ come from diffeomorphisms $X \rightarrow X$ ? No: for instance, translation along cotangent fibers is not induced by a diffeomorphism of the base manifold. A criterion for which symplectomorphisms arise as lifts of diffeomorphisms is discussed in Homework 3. 


\section{Homework 2: Symplectic Volume}

1. Given a vector space $V$, the exterior algebra of its dual space is

$$
\wedge^{*}\left(V^{*}\right)=\bigoplus_{k=0}^{\operatorname{dim} V} \wedge^{k}\left(V^{*}\right)
$$

where $\wedge^{k}\left(V^{*}\right)$ is the set of maps $\alpha: \overbrace{V \times \cdots \times V}^{k} \rightarrow \mathbb{R}$ which are linear in each entry, and for any permutation $\pi, \alpha\left(v_{\pi_{1}}, \ldots, v_{\pi_{k}}\right)=(\operatorname{sign} \pi) \cdot \alpha\left(v_{1}, \ldots, v_{k}\right)$. The elements of $\wedge^{k}\left(V^{*}\right)$ are known as skew-symmetric $k$-linear maps or $k$-forms on $V$.

(a) Show that any $\Omega \in \wedge^{2}\left(V^{*}\right)$ is of the form $\Omega=e_{1}^{*} \wedge f_{1}^{*}+\ldots+e_{n}^{*} \wedge$ $f_{n}^{*}$, where $u_{1}^{*}, \ldots, u_{k}^{*}, e_{1}^{*}, \ldots, e_{n}^{*}, f_{1}^{*}, \ldots, f_{n}^{*}$ is a basis of $V^{*}$ dual to the standard basis $(k+2 n=\operatorname{dim} V)$.

(b) In this language, a symplectic map $\Omega: V \times V \rightarrow \mathbb{R}$ is just a nondegenerate 2-form $\Omega \in \wedge^{2}\left(V^{*}\right)$, called a symplectic form on $V$.

Show that, if $\Omega$ is any symplectic form on a vector space $V$ of dimension $2 n$, then the $n$th exterior power $\Omega^{n}=\underbrace{\Omega \wedge \ldots \wedge \Omega}_{n}$ does not vanish.

(c) Deduce that the $n$th exterior power $\omega^{n}$ of any symplectic form $\omega$ on a $2 n$-dimensional manifold $M$ is a volume form. ${ }^{2}$

Hence, any symplectic manifold $(M, \omega)$ is canonically oriented by the symplectic structure. The form $\frac{\omega^{n}}{n !}$ is called the symplectic volume or the Liouville volume of $(M, \omega)$.

Does the Möbius strip support a symplectic structure?

(d) Conversely, given a 2-form $\Omega \in \wedge^{2}\left(V^{*}\right)$, show that, if $\Omega^{n} \neq 0$, then $\Omega$ is symplectic.

Hint: Standard form.

2. Let $(M, \omega)$ be a $2 n$-dimensional symplectic manifold, and let $\omega^{n}$ be the volume form obtained by wedging $\omega$ with itself $n$ times.

(a) Show that, if $M$ is compact, the de Rham cohomology class $\left[\omega^{n}\right] \in$ $H^{2 n}(M ; \mathbb{R})$ is non-zero.

Hint: Stokes' theorem.

(b) Conclude that $[\omega]$ itself is non-zero (in other words, that $\omega$ is not exact).

(c) Show that if $n>1$ there are no symplectic structures on the sphere $S^{2 n}$.

\footnotetext{
${ }^{2} \mathrm{~A}$ volume form is a nonvanishing form of top degree.
} 



\section{Part II}

\section{Symplectomorphisms}

Equivalence between symplectic manifolds is expressed by a symplectomorphism. By Weinstein's lagrangian creed [105], everything is a lagrangian manifold! We will study symplectomorphisms according to the creed.

\section{$3 \quad$ Lagrangian Submanifolds}

\subsection{Submanifolds}

Let $M$ and $X$ be manifolds with $\operatorname{dim} X<\operatorname{dim} M$.

Definition 3.1 A map $i: X \rightarrow M$ is an immersion if $d i_{p}: T_{p} X \rightarrow T_{i(p)} M$ is injective for any point $p \in X$.

An embedding is an immersion which is a homeomorphism onto its image. ${ }^{3}$

A closed embedding is a proper ${ }^{4}$ injective immersion.

Exercise. Show that a map $i: X \rightarrow M$ is a closed embedding if and only if $i$ is an embedding and its image $i(X)$ is closed in $M$.

Hints:

- If $i$ is injective and proper, then for any neighborhood $\mathcal{U}$ of $p \in X$, there is a neighborhood $\mathcal{V}$ of $i(p)$ such that $f^{-1}(\mathcal{V}) \subseteq \mathcal{U}$.

- On a Hausdorff space, any compact set is closed. On any topological space, a closed subset of a compact set is compact.

- An embedding is proper if and only if its image is closed.

Definition $3.2 A$ submanifold of $M$ is a manifold $X$ with a closed embedding $i: X \hookrightarrow M{ }^{5}$

Notation. Given a submanifold, we regard the embedding $i: X \hookrightarrow M$ as an inclusion, in order to identify points and tangent vectors:

$$
p=i(p) \quad \text { and } \quad T_{p} X=d i_{p}\left(T_{p} X\right) \subset T_{p} M .
$$

\footnotetext{
${ }^{3}$ The image has the topology induced by the target manifold.

${ }^{4} \mathrm{~A}$ map is proper if the preimage of any compact set is compact.

${ }^{5}$ When $X$ is an open subset of a manifold $M$, we refer to it as an open submanifold.
} 


\subsection{Lagrangian Submanifolds of $T^{*} X$}

Definition 3.3 Let $(M, \omega)$ be a $2 n$-dimensional symplectic manifold. A submanifold $Y$ of $M$ is a lagrangian submanifold if, at each $p \in Y, T_{p} Y$ is a lagrangian subspace of $T_{p} M$, i.e., $\left.\omega_{p}\right|_{T_{p}} Y \equiv 0$ and $\operatorname{dim} T_{p} Y=\frac{1}{2} \operatorname{dim} T_{p} M$. Equivalently, if $i: Y \hookrightarrow M$ is the inclusion map, then $Y$ is lagrangian if and only if $i^{*} \omega=0$ and $\operatorname{dim} Y=\frac{1}{2} \operatorname{dim} M$.

Let $X$ be an $n$-dimensional manifold, with $M=T^{*} X$ its cotangent bundle. If $x_{1}, \ldots, x_{n}$ are coordinates on $U \subseteq X$, with associated cotangent coordinates $x_{1}, \ldots, x_{n}, \xi_{1}, \ldots, \xi_{n}$ on $T^{*} U$, then the tautological 1-form on $T^{*} X$ is

$$
\alpha=\sum \xi_{i} d x_{i}
$$

and the canonical 2 -form on $T^{*} X$ is

$$
\omega=-d \alpha=\sum d x_{i} \wedge d \xi_{i} .
$$

The zero section of $T^{*} X$

$$
X_{0}:=\left\{(x, \xi) \in T^{*} X \mid \xi=0 \text { in } T_{x}^{*} X\right\}
$$

is an $n$-dimensional submanifold of $T^{*} X$ whose intersection with $T^{*} U$ is given by the equations $\xi_{1}=\ldots=\xi_{n}=0$. Clearly $\alpha=\sum \xi_{i} d x_{i}$ vanishes on $X_{0} \cap T^{*} U$. In particular, if $i_{0}: X_{0} \hookrightarrow T^{*} X$ is the inclusion map, we have $i_{0}^{*} \alpha=0$. Hence, $i_{0}^{*} \omega=i_{0}^{*} d \alpha=0$, and $X_{0}$ is lagrangian.

What are all the lagrangian submanifolds of $T^{*} X$ which are " $C^{1}$-close to $X_{0}$ "?

Let $X_{\mu}$ be (the image of) another section, that is, an $n$-dimensional submanifold of $T^{*} X$ of the form

$$
X_{\mu}=\left\{\left(x, \mu_{x}\right) \mid x \in X, \mu_{x} \in T_{x}^{*} X\right\}
$$

where the covector $\mu_{x}$ depends smoothly on $x$, and $\mu: X \rightarrow T^{*} X$ is a de Rham 1-form. Relative to the inclusion $i: X_{\mu} \hookrightarrow T^{*} X$ and the cotangent projection $\pi: T^{*} X \rightarrow X, X_{\mu}$ is of the form ( $\star$ ) if and only if $\pi \circ i: X_{\mu} \rightarrow X$ is a diffeomorphism.

When is such an $X_{\mu}$ lagrangian?

Proposition 3.4 Let $X_{\mu}$ be of the form $(\star)$, and let $\mu$ be the associated de Rham 1 -form. Denote by $s_{\mu}: X \rightarrow T^{*} X, x \mapsto\left(x, \mu_{x}\right)$, the 1 -form $\mu$ regarded exclusively as a map. Notice that the image of $s_{\mu}$ is $X_{\mu}$. Let $\alpha$ be the tautological 1-form on $T^{*} X$. Then

$$
s_{\mu}^{*} \alpha=\mu .
$$

Proof. By definition of $\alpha$ (previous lecture), $\alpha_{p}=\left(d \pi_{p}\right)^{*} \xi$ at $p=(x, \xi) \in M$. For $p=s_{\mu}(x)=\left(x, \mu_{x}\right)$, we have $\alpha_{p}=\left(d \pi_{p}\right)^{*} \mu_{x}$. Then

$$
\left(s_{\mu}^{*} \alpha\right)_{x}=\left(d s_{\mu}\right)_{x}^{*} \alpha_{p}=\left(d s_{\mu}\right)_{x}^{*}\left(d \pi_{p}\right)^{*} \mu_{x}=\left(d(\underbrace{\pi \circ s_{\mu}}_{\mathrm{id} X})\right)_{x}^{*} \mu_{x}=\mu_{x} .
$$


Suppose that $X_{\mu}$ is an $n$-dimensional submanifold of $T^{*} X$ of the form $(\star)$, with associated de Rham 1-form $\mu$. Then $s_{\mu}: X \rightarrow T^{*} X$ is an embedding with image $X_{\mu}$, and there is a diffeomorphism $\tau: X \rightarrow X_{\mu}, \tau(x):=\left(x, \mu_{x}\right)$, such that the following diagram commutes.

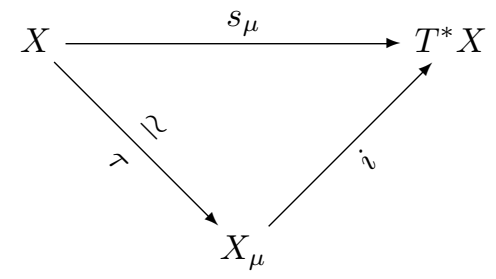

We want to express the condition of $X_{\mu}$ being lagrangian in terms of the form $\mu$ :

$$
\begin{aligned}
X_{\mu} \text { is lagrangian } & \Longleftrightarrow i^{*} d \alpha=0 \\
& \Longleftrightarrow \tau^{*} i^{*} d \alpha=0 \\
& \Longleftrightarrow s_{\mu}^{*} d \alpha=0 \\
& \Longleftrightarrow d s_{\mu}^{*} \alpha=0 \\
& \Longleftrightarrow d \mu=0 \\
& \Longleftrightarrow \mu \text { is closed } .
\end{aligned}
$$

Therefore, there is a one-to-one correspondence between the set of lagrangian submanifolds of $T^{*} X$ of the form $(\star)$ and the set of closed 1-forms on $X$.

When $X$ is simply connected, $H_{\text {deRham }}^{1}(X)=0$, so every closed 1 -form $\mu$ is equal to $d f$ for some $f \in C^{\infty}(X)$. Any such primitive $f$ is then called a generating function for the lagrangian submanifold $X_{\mu}$ associated to $\mu$. (Two functions generate the same lagrangian submanifold if and only if they differ by a locally constant function.) On arbitrary manifolds $X$, functions $f \in C^{\infty}(X)$ originate lagrangian submanifolds as images of $d f$.

Exercise. Check that, if $X$ is compact (and not just one point) and $f \in C^{\infty}(X)$, then $\#\left(X_{d f} \cap X_{0}\right) \geq 2$.

There are lots of lagrangian submanifolds of $T^{*} X$ not covered by the description in terms of closed 1 -forms, starting with the cotangent fibers.

\subsection{Conormal Bundles}

Let $S$ be any $k$-dimensional submanifold of an $n$-dimensional manifold $X$.

Definition 3.5 The conormal space at $x \in S$ is

$$
N_{x}^{*} S=\left\{\xi \in T_{x}^{*} X \mid \xi(v)=0, \text { for all } v \in T_{x} S\right\} .
$$

The conormal bundle of $S$ is

$$
N^{*} S=\left\{(x, \xi) \in T^{*} X \mid x \in S, \xi \in N_{x}^{*} S\right\} .
$$


Exercise. The conormal bundle $N^{*} S$ is an $n$-dimensional submanifold of $T^{*} X$.

Hint: Use coordinates on $X$ adapted $^{6}$ to $S$.

Proposition 3.6 Let $i: N^{*} S \hookrightarrow T^{*} X$ be the inclusion, and let $\alpha$ be the tautological 1-form on $T^{*} X$. Then

$$
i^{*} \alpha=0
$$

Proof. Let $\left(\mathcal{U}, x_{1}, \ldots, x_{n}\right)$ be a coordinate system on $X$ centered at $x \in S$ and adapted to $S$, so that $\mathcal{U} \cap S$ is described by $x_{k+1}=\ldots=x_{n}=0$. Let $\left(T^{*} \mathcal{U}, x_{1}, \ldots, x_{n}, \xi_{1}, \ldots, \xi_{n}\right)$ be the associated cotangent coordinate system. The submanifold $N^{*} S \cap T^{*} \mathcal{U}$ is then described by

$$
x_{k+1}=\ldots=x_{n}=0 \quad \text { and } \quad \xi_{1}=\ldots=\xi_{k}=0 .
$$

Since $\alpha=\sum \xi_{i} d x_{i}$ on $T^{*} \mathcal{U}$, we conclude that, at $p \in N^{*} S$,

$$
\left(i^{*} \alpha\right)_{p}=\left.\alpha_{p}\right|_{T_{p}\left(N^{*} S\right)}=\left.\sum_{i>k} \xi_{i} d x_{i}\right|_{\operatorname{span}\left\{\frac{\partial}{\partial x_{i}}, i \leq k\right\}}=0 .
$$

Corollary 3.7 For any submanifold $S \subset X$, the conormal bundle $N^{*} S$ is a lagrangian submanifold of $T^{*} X$.

Taking $S=\{x\}$ to be one point, the conormal bundle $L=N^{*} S=T_{x}^{*} X$ is a cotangent fiber. Taking $S=X$, the conormal bundle $L=X_{0}$ is the zero section of $T^{*} X$.

\subsection{Application to Symplectomorphisms}

Let $\left(M_{1}, \omega_{1}\right)$ and $\left(M_{2}, \omega_{2}\right)$ be two $2 n$-dimensional symplectic manifolds. Given a diffeomorphism $\varphi: M_{1} \stackrel{\simeq}{\longrightarrow} M_{2}$, when is it a symplectomorphism? (I.e., when is $\varphi^{*} \omega_{2}=\omega_{1}$ ?)

Consider the two projection maps

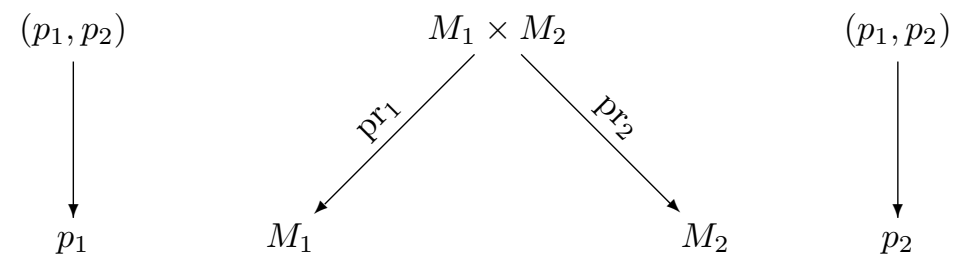

\footnotetext{
${ }^{6}$ A coordinate chart $\left(\mathcal{U}, x_{1}, \ldots, x_{n}\right)$ on $X$ is adapted to a $k$-dimensional submanifold $S$ if $S \cap \mathcal{U}$ is described by $x_{k+1}=\ldots=x_{n}=0$.
} 
Then $\omega=\left(\operatorname{pr}_{1}\right)^{*} \omega_{1}+\left(\operatorname{pr}_{2}\right)^{*} \omega_{2}$ is a 2 -form on $M_{1} \times M_{2}$ which is closed,

$$
d \omega=\left(\mathrm{pr}_{1}\right)^{*} \underbrace{d \omega_{1}}_{0}+\left(\mathrm{pr}_{2}\right)^{*} \underbrace{d \omega_{2}}_{0}=0
$$

and symplectic,

$$
\omega^{2 n}=\left(\begin{array}{c}
2 n \\
n
\end{array}\right)\left(\left(\operatorname{pr}_{1}\right)^{*} \omega_{1}\right)^{n} \wedge\left(\left(\operatorname{pr}_{2}\right)^{*} \omega_{2}\right)^{n} \neq 0
$$

More generally, if $\lambda_{1}, \lambda_{2} \in \mathbb{R} \backslash\{0\}$, then $\lambda_{1}\left(\mathrm{pr}_{1}\right)^{*} \omega_{1}+\lambda_{2}\left(\mathrm{pr}_{2}\right)^{*} \omega_{2}$ is also a symplectic form on $M_{1} \times M_{2}$. Take $\lambda_{1}=1, \lambda_{2}=-1$ to obtain the twisted product form on $M_{1} \times M_{2}$ :

$$
\widetilde{\omega}=\left(\mathrm{pr}_{1}\right)^{*} \omega_{1}-\left(\mathrm{pr}_{2}\right)^{*} \omega_{2}
$$

The graph of a diffeomorphism $\varphi: M_{1} \stackrel{\simeq}{\longrightarrow} M_{2}$ is the $2 n$-dimensional submanifold of $M_{1} \times M_{2}$ :

$$
\Gamma_{\varphi}:=\operatorname{Graph} \varphi=\left\{(p, \varphi(p)) \mid p \in M_{1}\right\} .
$$

The submanifold $\Gamma_{\varphi}$ is an embedded image of $M_{1}$ in $M_{1} \times M_{2}$, the embedding being the map

$$
\begin{aligned}
& \gamma: M_{1} \longrightarrow M_{1} \times M_{2} \\
& p \longmapsto(p, \varphi(p)) .
\end{aligned}
$$

Proposition 3.8 A diffeomorphism $\varphi$ is a symplectomorphism if and only if $\Gamma_{\varphi}$ is a lagrangian submanifold of $\left(M_{1} \times M_{2}, \widetilde{\omega}\right)$.

Proof. The graph $\Gamma_{\varphi}$ is lagrangian if and only if $\gamma^{*} \widetilde{\omega}=0$. But

$$
\begin{aligned}
\gamma^{*} \widetilde{\omega} & =\gamma^{*} \operatorname{pr}_{1}^{*} \omega_{1}-\gamma^{*} \operatorname{pr}_{2}^{*} \omega_{2} \\
& =\left(\operatorname{pr}_{1} \circ \gamma\right)^{*} \omega_{1}-\left(\operatorname{pr}_{2} \circ \gamma\right)^{*} \omega_{2}
\end{aligned}
$$

and $\mathrm{pr}_{1} \circ \gamma$ is the identity map on $M_{1}$ whereas $\operatorname{pr}_{2} \circ \gamma=\varphi$. Therefore,

$$
\gamma^{*} \widetilde{\omega}=0 \quad \Longleftrightarrow \quad \varphi^{*} \omega_{2}=\omega_{1}
$$




\section{Homework 3:}

\section{Tautological Form and Symplectomorphisms}

This set of problems is from [53].

1. Let $(M, \omega)$ be a symplectic manifold, and let $\alpha$ be a 1 -form such that

$$
\omega=-d \alpha .
$$

Show that there exists a unique vector field $v$ such that its interior product with $\omega$ is $\alpha$, i.e., $\imath_{v} \omega=-\alpha$.

Prove that, if $g$ is a symplectomorphism which preserves $\alpha$ (that is, $g^{*} \alpha=\alpha$ ), then $g$ commutes with the one-parameter group of diffeomorphisms generated by $v$, i.e.,

$$
(\exp t v) \circ g=g \circ(\exp t v) .
$$

Hint: Recall that, for $p \in M,(\exp t v)(p)$ is the unique curve in $M$ solving the ordinary differential equation

$$
\left\{\begin{array}{l}
\frac{d}{d t}(\exp t v(p))=v(\exp t v(p)) \\
\left.(\exp t v)(p)\right|_{t=0}=p
\end{array}\right.
$$

for $t$ in some neighborhood of 0 . Show that $g \circ(\exp t v) \circ g^{-1}$ is the one-parameter group of diffeomorphisms generated by $g_{*} v$. (The push-forward of $v$ by $g$ is defined by $\left(g_{*} v\right)_{g(p)}=d g_{p}\left(v_{p}\right)$.) Finally check that $g$ preserves $v$ (that is, $g_{*} v=v$ ).

2. Let $X$ be an arbitrary $n$-dimensional manifold, and let $M=T^{*} X$. Let $\left(\mathcal{U}, x_{1}, \ldots, x_{n}\right)$ be a coordinate system on $X$, and let $x_{1}, \ldots, x_{n}, \xi_{1}, \ldots, \xi_{n}$ be the corresponding coordinates on $T^{*} \mathcal{U}$.

Show that, when $\alpha$ is the tautological 1 -form on $M$ (which, in these coordinates, is $\left.\sum \xi_{i} d x_{i}\right)$, the vector field $v$ in the previous exercise is just the vector field $\sum \xi_{i} \frac{\partial}{\partial \xi_{i}}$.

Let $\exp t v,-\infty<t<\infty$, be the one-parameter group of diffeomorphisms generated by $v$.

Show that, for every point $p=(x, \xi)$ in $M$,

$$
(\exp t v)(p)=p_{t} \quad \text { where } \quad p_{t}=\left(x, e^{t} \xi\right) .
$$


3. Let $M$ be as in exercise 2 .

Show that, if $g$ is a symplectomorphism of $M$ which preserves $\alpha$, then

$$
g(x, \xi)=(y, \eta) \quad \Longrightarrow \quad g(x, \lambda \xi)=(y, \lambda \eta)
$$

for all $(x, \xi) \in M$ and $\lambda \in \mathbb{R}$.

Conclude that $g$ has to preserve the cotangent fibration, i.e., show that there exists a diffeomorphism $f: X \rightarrow X$ such that $\pi \circ g=f \circ \pi$, where $\pi: M \rightarrow X$ is the projection map $\pi(x, \xi)=x$.

Finally prove that $g=f_{\#}$, the map $f_{\#}$ being the symplectomorphism of $M$ lifting $f$.

Hint: Suppose that $g(p)=q$ where $p=(x, \xi)$ and $q=(y, \eta)$.

Combine the identity

$$
\left(d g_{p}\right)^{*} \alpha_{q}=\alpha_{p}
$$

with the identity

$$
d \pi_{q} \circ d g_{p}=d f_{x} \circ d \pi_{p} .
$$

(The first identity expresses the fact that $g^{*} \alpha=\alpha$, and the second identity is obtained by differentiating both sides of the equation $\pi \circ g=f \circ \pi$ at $p$.)

4. Let $M$ be as in exercise 2, and let $h$ be a smooth function on $X$. Define $\tau_{h}: M \rightarrow M$ by setting

$$
\tau_{h}(x, \xi)=\left(x, \xi+d h_{x}\right) .
$$

Prove that

$$
\tau_{h}^{*} \alpha=\alpha+\pi^{*} d h
$$

where $\pi$ is the projection map

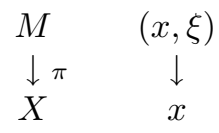

Deduce that

$$
\tau_{h}^{*} \omega=\omega,
$$

i.e., that $\tau_{h}$ is a symplectomorphism. 


\section{Generating Functions}

\subsection{Constructing Symplectomorphisms}

Let $X_{1}, X_{2}$ be $n$-dimensional manifolds, with cotangent bundles $M_{1}=T^{*} X_{1}$, $M_{2}=T^{*} X_{2}$, tautological 1-forms $\alpha_{1}, \alpha_{2}$, and canonical 2-forms $\omega_{1}, \omega_{2}$.

Under the natural identification

$$
M_{1} \times M_{2}=T^{*} X_{1} \times T^{*} X_{2} \simeq T^{*}\left(X_{1} \times X_{2}\right),
$$

the tautological 1-form on $T^{*}\left(X_{1} \times X_{2}\right)$ is

$$
\alpha=\left(\mathrm{pr}_{1}\right)^{*} \alpha_{1}+\left(\mathrm{pr}_{2}\right)^{*} \alpha_{2},
$$

where $\mathrm{pr}_{i}: M_{1} \times M_{2} \rightarrow M_{i}, i=1,2$ are the two projections. The canonical 2-form on $T^{*}\left(X_{1} \times X_{2}\right)$ is

$$
\omega=-d \alpha=-d \operatorname{pr}_{1}^{*} \alpha_{1}-d \operatorname{pr}_{2}^{*} \alpha_{2}=\operatorname{pr}_{1}^{*} \omega_{1}+\operatorname{pr}_{2}^{*} \omega_{2} .
$$

In order to describe the twisted form $\widetilde{\omega}=\operatorname{pr}_{1}^{*} \omega_{1}-\operatorname{pr}_{2}^{*} \omega_{2}$, we define an involution of $M_{2}=T^{*} X_{2}$ by

$$
\begin{aligned}
\sigma_{2}: \quad & M_{2} \\
\left(x_{2}, \xi_{2}\right) & \longmapsto\left(x_{2},-\xi_{2}\right)
\end{aligned}
$$

which yields $\sigma_{2}^{*} \alpha_{2}=-\alpha_{2}$. Let $\sigma=\operatorname{id}_{M_{1}} \times \sigma_{2}: M_{1} \times M_{2} \rightarrow M_{1} \times M_{2}$. Then

$$
\sigma^{*} \widetilde{\omega}=\operatorname{pr}_{1}^{*} \omega_{1}+\operatorname{pr}_{2}^{*} \omega_{2}=\omega
$$

If $Y$ is a lagrangian submanifold of $\left(M_{1} \times M_{2}, \omega\right)$, then its "twist" $Y^{\sigma}:=\sigma(Y)$ is a lagrangian submanifold of $\left(M_{1} \times M_{2}, \widetilde{\omega}\right)$.

Recipe for producing symplectomorphisms $M_{1}=T^{*} X_{1} \rightarrow M_{2}=T^{*} X_{2}$ :

1. Start with a lagrangian submanifold $Y$ of $\left(M_{1} \times M_{2}, \omega\right)$.

2. Twist it to obtain a lagrangian submanifold $Y^{\sigma}$ of $\left(M_{1} \times M_{2}, \widetilde{\omega}\right)$.

3. Check whether $Y^{\sigma}$ is the graph of some diffeomorphism $\varphi: M_{1} \rightarrow M_{2}$.

4. If it is, then $\varphi$ is a symplectomorphism (by Proposition 3.8).

Let $i: Y^{\sigma} \hookrightarrow M_{1} \times M_{2}$ be the inclusion map

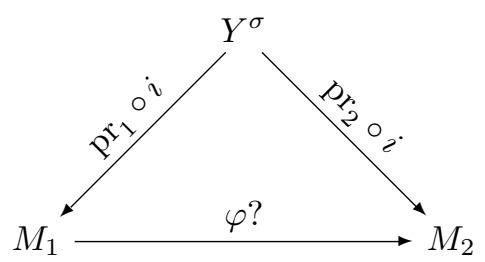

Step 3 amounts to checking whether $\mathrm{pr}_{1} \circ i$ and $\mathrm{pr}_{2} \circ i$ are diffeomorphisms. If yes, then $\varphi:=\left(\operatorname{pr}_{2} \circ i\right) \circ\left(\operatorname{pr}_{1} \circ i\right)^{-1}$ is a diffeomorphism.

In order to obtain lagrangian submanifolds of $M_{1} \times M_{2} \simeq T^{*}\left(X_{1} \times X_{2}\right)$, we can use the method of generating functions. 


\subsection{Method of Generating Functions}

For any $f \in C^{\infty}\left(X_{1} \times X_{2}\right)$, $d f$ is a closed 1-form on $X_{1} \times X_{2}$. The lagrangian submanifold generated by $f$ is

$$
Y_{f}:=\left\{\left((x, y),(d f)_{(x, y)}\right) \mid(x, y) \in X_{1} \times X_{2}\right\}
$$

We adopt the notation

$$
\begin{aligned}
& d_{x} f:=(d f)_{(x, y)} \text { projected to } T_{x}^{*} X_{1} \times\{0\} \\
& d_{y} f:=(d f)_{(x, y)} \text { projected to }\{0\} \times T_{y}^{*} X_{2}
\end{aligned}
$$

which enables us to write

$$
Y_{f}=\left\{\left(x, y, d_{x} f, d_{y} f\right) \mid(x, y) \in X_{1} \times X_{2}\right\}
$$

and

$$
Y_{f}^{\sigma}=\left\{\left(x, y, d_{x} f,-d_{y} f\right) \mid(x, y) \in X_{1} \times X_{2}\right\} .
$$

When $Y_{f}^{\sigma}$ is in fact the graph of a diffeomorphism $\varphi: M_{1} \rightarrow M_{2}$, we call $\varphi$ the symplectomorphism generated by $f$, and call $f$ the generating function, of $\varphi: M_{1} \rightarrow M_{2}$.

So when is $Y_{f}^{\sigma}$ the graph of a diffeomorphism $\varphi: M_{1} \rightarrow M_{2}$ ?

Let $\left(\mathcal{U}_{1}, x_{1}, \ldots, x_{n}\right),\left(\mathcal{U}_{2}, y_{1}, \ldots, y_{n}\right)$ be coordinate charts for $X_{1}, X_{2}$, with associated charts $\left(T^{*} \mathcal{U}_{1}, x_{1}, \ldots, x_{n}, \xi_{1}, \ldots, \xi_{n}\right),\left(T^{*} \mathcal{U}_{2}, y_{1}, \ldots, y_{n}, \eta_{1}, \ldots, \eta_{n}\right)$ for $M_{1}, M_{2}$. The set $Y_{f}^{\sigma}$ is the graph of $\varphi: M_{1} \rightarrow M_{2}$ if and only if, for any $(x, \xi) \in M_{1}$ and $(y, \eta) \in M_{2}$, we have

$$
\varphi(x, \xi)=(y, \eta) \Longleftrightarrow \xi=d_{x} f \text { and } \eta=-d_{y} f
$$

Therefore, given a point $(x, \xi) \in M_{1}$, to find its image $(y, \eta)=\varphi(x, \xi)$ we must solve the "Hamilton" equations

$$
\left\{\begin{aligned}
\xi_{i} & = & \frac{\partial f}{\partial x_{i}}(x, y) \\
\eta_{i} & = & -\frac{\partial f}{\partial y_{i}}(x, y) .
\end{aligned}\right.
$$

If there is a solution $y=\varphi_{1}(x, \xi)$ of $(\star)$, we may feed it to $(\star \star)$ thus obtaining $\eta=\varphi_{2}(x, \xi)$, so that $\varphi(x, \xi)=\left(\varphi_{1}(x, \xi), \varphi_{2}(x, \xi)\right)$. Now by the implicit function theorem, in order to solve $(\star)$ locally for $y$ in terms of $x$ and $\xi$, we need the condition

$$
\operatorname{det}\left[\frac{\partial}{\partial y_{j}}\left(\frac{\partial f}{\partial x_{i}}\right)\right]_{i, j=1}^{n} \neq 0 \text {. }
$$

This is a necessary local condition for $f$ to generate a symplectomorphism $\varphi$. Locally this is also sufficient, but globally there is the usual bijectivity issue. 
Example. Let $X_{1}=\mathcal{U}_{1} \simeq \mathbb{R}^{n}, X_{2}=\mathcal{U}_{2} \simeq \mathbb{R}^{n}$, and $f(x, y)=-\frac{|x-y|^{2}}{2}$, the square of euclidean distance up to a constant.

The "Hamilton" equations are

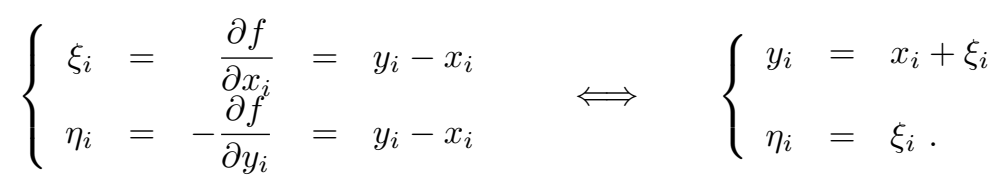

The symplectomorphism generated by $f$ is

$$
\varphi(x, \xi)=(x+\xi, \xi) .
$$

If we use the euclidean inner product to identify $T^{*} \mathbb{R}^{n}$ with $T \mathbb{R}^{n}$, and hence regard $\varphi$ as $\widetilde{\varphi}: T \mathbb{R}^{n} \rightarrow T \mathbb{R}^{n}$ and interpret $\xi$ as the velocity vector, then the symplectomorphism $\varphi$ corresponds to free translational motion in euclidean space.

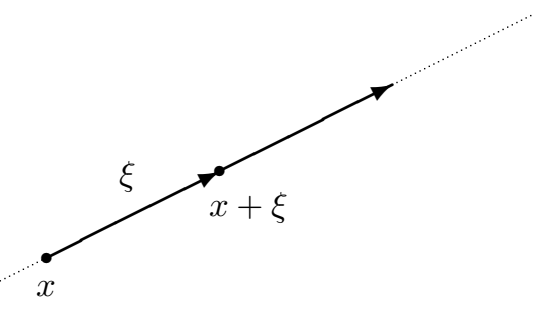

\subsection{Application to Geodesic Flow}

Let $V$ be an $n$-dimensional vector space. A positive inner product $G$ on $V$ is a bilinear map $G: V \times V \rightarrow \mathbb{R}$ which is

$$
\begin{array}{lll}
\text { symmetric : } & G(v, w)=G(w, v), & \text { and } \\
\text { positive-definite : } & G(v, v)>0 \quad \text { when } & v \neq 0 .
\end{array}
$$

Definition 4.1 A riemannian metric on a manifold $X$ is a function $g$ which assigns to each point $x \in X$ a positive inner product $g_{x}$ on $T_{x} X$.

A riemannian metric $g$ is smooth if for every smooth vector field $v: X \rightarrow T X$ the real-valued function $x \mapsto g_{x}\left(v_{x}, v_{x}\right)$ is a smooth function on $X$.

Definition $4.2 A$ riemannian manifold $(X, g)$ is a manifold $X$ equipped with a smooth riemannian metric $g$. 
The arc-length of a piecewise smooth curve $\gamma:[a, b] \rightarrow X$ on a riemannian manifold $(X, g)$ is

$$
\int_{a}^{b} \sqrt{g_{\gamma(t)}\left(\frac{d \gamma}{d t}, \frac{d \gamma}{d t}\right)} d t
$$

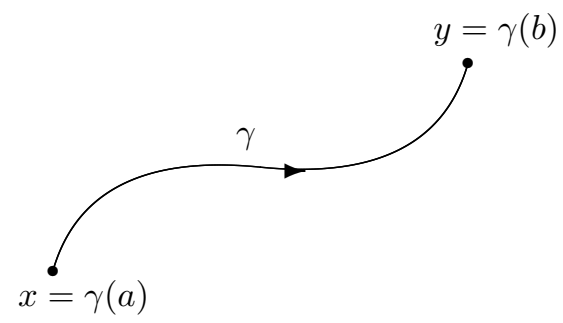

Definition 4.3 The riemannian distance between two points $x$ and $y$ of a connected riemannian manifold $(X, g)$ is the infimum $d(x, y)$ of the set of all arc-lengths for piecewise smooth curves joining $x$ to $y$.

A smooth curve joining $x$ to $y$ is a minimizing geodesic ${ }^{7}$ if its arc-length is the riemannian distance $d(x, y)$.

A riemannian manifold $(X, g)$ is geodesically convex if every point $x$ is joined to every other point $y$ by a unique minimizing geodesic.

Example. On $X=\mathbb{R}^{n}$ with $T X \simeq \mathbb{R}^{n} \times \mathbb{R}^{n}$, let $g_{x}(v, w)=\langle v, w\rangle, g_{x}(v, v)=$ $|v|^{2}$, where $\langle\cdot, \cdot\rangle$ is the euclidean inner product, and $|\cdot|$ is the euclidean norm. Then $\left(\mathbb{R}^{n},\langle\cdot, \cdot\rangle\right)$ is a geodesically convex riemannian manifold, and the riemannian distance is the usual euclidean distance $d(x, y)=|x-y|$.

Suppose that $(X, g)$ is a geodesically convex riemannian manifold. Consider the function

$$
f: X \times X \longrightarrow \mathbb{R}, \quad f(x, y)=-\frac{d(x, y)^{2}}{2} .
$$

What is the symplectomorphism $\varphi: T^{*} X \rightarrow T^{*} X$ generated by $f$ ?

The metric $g_{x}: T_{x} X \times T_{x} X \rightarrow \mathbb{R}$ induces an identification

$$
\begin{aligned}
\tilde{g}_{x}: T_{x} X & \stackrel{\simeq}{\longmapsto} T_{x}^{*} X \\
v & \longmapsto g_{x}(v, \cdot)
\end{aligned}
$$

Use $\widetilde{g}$ to translate $\varphi$ into a map $\widetilde{\varphi}: T X \rightarrow T X$.

We need to solve

$$
\left\{\begin{array}{llll}
\widetilde{g}_{x}(v) & =\xi_{i} & = & d_{x} f(x, y) \\
\widetilde{g}_{y}(w) & =\eta_{i} & = & -d_{y} f(x, y)
\end{array}\right.
$$

\footnotetext{
${ }^{7}$ In riemannian geometry, a geodesic is a curve which locally minimizes distance and whose velocity is constant.
} 
for $(y, \eta)$ in terms of $(x, \xi)$ in order to find $\varphi$, or, equivalently, for $(y, w)$ in terms $(x, v)$ in order to find $\widetilde{\varphi}$.

Let $\gamma$ be the geodesic with initial conditions $\gamma(0)=x$ and $\frac{d \gamma}{d t}(0)=v$.

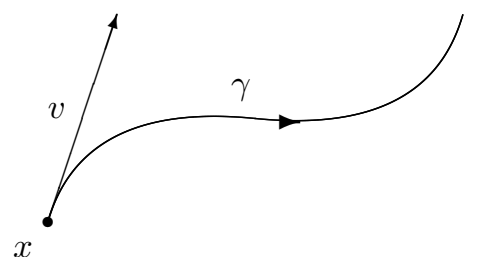

Then the symplectomorphism $\varphi$ corresponds to the map

$$
\begin{aligned}
\widetilde{\varphi}: T X & \longrightarrow T X \\
(x, v) & \longmapsto\left(\gamma(1), \frac{d \gamma}{d t}(1)\right) .
\end{aligned}
$$

This is called the geodesic flow on $X$ (see Homework 4). 


\section{Homework 4: Geodesic Flow}

This set of problems is adapted from [53].

Let $(X, g)$ be a riemannian manifold. The arc-length of a smooth curve $\gamma$ : $[a, b] \rightarrow X$ is

arc-length of $\gamma:=\int_{a}^{b}\left|\frac{d \gamma}{d t}\right| d t, \quad$ where $\quad\left|\frac{d \gamma}{d t}\right|:=\sqrt{g_{\gamma(t)}\left(\frac{d \gamma}{d t}, \frac{d \gamma}{d t}\right)}$.

1. Show that the arc-length of $\gamma$ is independent of the parametrization of $\gamma$, i.e., show that, if we reparametrize $\gamma$ by $\tau:\left[a^{\prime}, b^{\prime}\right] \rightarrow[a, b]$, the new curve $\gamma^{\prime}=\gamma \circ \tau:\left[a^{\prime}, b^{\prime}\right] \rightarrow X$ has the same arc-length. A curve $\gamma$ is called a curve of constant velocity when $\left|\frac{d \gamma}{d t}\right|$ is independent of $t$. Show that, given any curve $\gamma:[a, b] \rightarrow X$ (with $\frac{d \gamma}{d t}$ never vanishing), there is a reparametrization $\tau:[a, b] \rightarrow[a, b]$ such that $\gamma \circ \tau:[a, b] \rightarrow X$ is of constant velocity.

2. Given a smooth curve $\gamma:[a, b] \rightarrow X$, the action of $\gamma$ is $\mathcal{A}(\gamma):=\int_{a}^{b}\left|\frac{d \gamma}{d t}\right|^{2} d t$. Show that, among all curves joining $x$ to $y, \gamma$ minimizes the action if and only if $\gamma$ is of constant velocity and $\gamma$ minimizes arc-length.

Hint: Suppose that $\gamma$ is of constant velocity, and let $\tau:[a, b] \rightarrow[a, b]$ be a reparametrization. Show that $\mathcal{A}(\gamma \circ \tau) \geq \mathcal{A}(\gamma)$, with equality only when $\tau=$ identity.

3. Assume that $(X, g)$ is geodesically convex, that is, any two points $x, y \in X$ are joined by a unique (up to reparametrization) minimizing geodesic; its arc-length $d(x, y)$ is called the riemannian distance between $x$ and $y$.

Assume also that $(X, g)$ is geodesically complete, that is, every geodesic can be extended indefinitely. Given $(x, v) \in T X$, let $\exp (x, v): \mathbb{R} \rightarrow X$ be the unique minimizing geodesic of constant velocity with initial conditions $\exp (x, v)(0)=x$ and $\frac{d \exp (x, v)}{d t}(0)=v$.

Consider the function $f: X \times X \rightarrow \mathbb{R}$ given by $f(x, y)=-\frac{1}{2} \cdot d(x, y)^{2}$. Let $d_{x} f$ and $d_{y} f$ be the components of $d f_{(x, y)}$ with respect to $T_{(x, y)}^{*}(X \times X) \simeq$ $T_{x}^{*} X \times T_{y}^{*} X$. Recall that, if

$$
\Gamma_{f}^{\sigma}=\left\{\left(x, y, d_{x} f,-d_{y} f\right) \mid(x, y) \in X \times X\right\}
$$

is the graph of a diffeomorphism $f: T^{*} X \rightarrow T^{*} X$, then $f$ is the symplectomorphism generated by $f$. In this case, $f(x, \xi)=(y, \eta)$ if and only if $\xi=d_{x} f$ and $\eta=-d_{y} f$.

Show that, under the identification of $T X$ with $T^{*} X$ by $g$, the symplectomorphism generated by $f$ coincides with the map $T X \rightarrow T X,(x, v) \mapsto$ $\exp (x, v)(1)$. 
Hint: The metric $g$ provides the identifications $T_{x} X v \simeq \xi(\cdot)=g_{x}(v, \cdot) \in$ $T_{x}^{*} X$. We need to show that, given $(x, v) \in T X$, the unique solution of

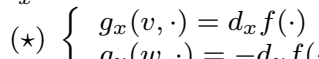

(*) $\left\{\begin{array}{l}g_{x}(v, \cdot)=d_{x} f(\cdot) \\ g_{y}(w, \cdot)=-d_{y} f(\cdot)\end{array}\right.$

is $(y, w)=\left(\exp (x, v)(1), d \frac{\exp (x, v)}{d t}(1)\right)$.

Look up the Gauss lemma in a book on riemannian geometry. It asserts that geodesics are orthogonal to the level sets of the distance function.

To solve the first line in $(\star)$ for $y$, evaluate both sides at $v=\frac{d \exp (x, v)}{d t}(0)$. Conclude that $y=\exp (x, v)(1)$. Check that $d_{x} f\left(v^{\prime}\right)=0$ for vectors $v^{\prime} \in T_{x} X$ orthogonal to $v$ (that is, $g_{x}\left(v, v^{\prime}\right)=0$ ); this is a consequence of $f(x, y)$ being the arc-length of a minimizing geodesic, and it suffices to check locally.

The vector $w$ is obtained from the second line of $(\star)$. Compute $-d_{y} f\left(\frac{d \exp (x, v)}{d t}(1)\right)$. Then evaluate $-d_{y} f$ at vectors $w^{\prime} \in T_{y} X$ orthogonal to $\frac{d \exp (x, v)}{d t}(1)$; this pairing is again 0 because $f(x, y)$ is the arc-length of a minimizing geodesic. Conclude, using the nondegeneracy of $g$, that $w=\frac{d \exp (x, v)}{d t}(1)$. For both steps, it might be useful to recall that, given a function $\varphi: X \stackrel{d t}{\rightarrow} \mathbb{R}$ and a tangent vector $v \in T_{x} X$, we have $d \varphi_{x}(v)=\frac{d}{d u}[\varphi(\exp (x, v)(u))]_{u=0}$. 


\section{Recurrence}

\subsection{Periodic Points}

Let $X$ be an $n$-dimensional manifold. Let $M=T^{*} X$ be its cotangent bundle with canonical symplectic form $\omega$.

Suppose that we are given a smooth function $f: X \times X \rightarrow \mathbb{R}$ which generates a symplectomorphism $\varphi: M \rightarrow M, \varphi\left(x, d_{x} f\right)=\left(y,-d_{y} f\right)$, by the recipe of the previous lecture.

What are the fixed points of $\varphi$ ?

Define $\psi: X \rightarrow \mathbb{R}$ by $\psi(x)=f(x, x)$.

Proposition 5.1 There is a one-to-one correspondence between the fixed points of $\varphi$ and the critical points of $\psi$.

Proof. At $x_{0} \in X, d_{x_{0}} \psi=\left.\left(d_{x} f+d_{y} f\right)\right|_{(x, y)=\left(x_{0}, x_{0}\right)}$. Let $\xi=\left.d_{x} f\right|_{(x, y)=\left(x_{0}, x_{0}\right)}$.

$x_{0}$ is a critical point of $\psi \Longleftrightarrow d_{x_{0}} \psi=\left.0 \Longleftrightarrow d_{y} f\right|_{(x, y)=\left(x_{0}, x_{0}\right)}=-\xi$.

Hence, the point in $\Gamma_{f}^{\sigma}$ corresponding to $(x, y)=\left(x_{0}, x_{0}\right)$ is $\left(x_{0}, x_{0}, \xi, \xi\right)$. But $\Gamma_{f}^{\sigma}$ is the graph of $\varphi$, so $\varphi\left(x_{0}, \xi\right)=\left(x_{0}, \xi\right)$ is a fixed point. This argument also works backwards.

Consider the iterates of $\varphi$,

$$
\varphi^{(N)}=\underbrace{\varphi \circ \varphi \circ \ldots \circ \varphi}_{N}: M \longrightarrow M, \quad N=1,2, \ldots,
$$

each of which is a symplectomorphism of $M$. According to the previous proposition, if $\varphi^{(N)}: M \rightarrow M$ is generated by $f^{(N)}$, then there is a correspondence

$$
\left\{\text { fixed points of } \varphi^{(N)}\right\} \stackrel{1-1}{\longleftrightarrow}\left\{\begin{array}{c}
\text { critical points of } \\
\psi^{(N)}: X \rightarrow \mathbb{R}, \psi^{(N)}(x)=f^{(N)}(x, x)
\end{array}\right\}
$$

Knowing that $\varphi$ is generated by $f$, does $\varphi^{(2)}$ have a generating function? The answer is a partial yes:

Fix $x, y \in X$. Define a map

$$
\begin{aligned}
X & \longrightarrow \mathbb{R} \\
z & \longmapsto f(x, z)+f(z, y) .
\end{aligned}
$$

Suppose that this map has a unique critical point $z_{0}$, and that $z_{0}$ is nondegenerate. Let

$$
f^{(2)}(x, y):=f\left(x, z_{0}\right)+f\left(z_{0}, y\right)
$$


Proposition 5.2 The function $f^{(2)}: X \times X \rightarrow \mathbb{R}$ is smooth and is a generating function for $\varphi^{(2)}$ if we assume that, for each $\xi \in T_{x}^{*} X$, there is a unique $y \in X$ for which $d_{x} f^{(2)}=\xi$.

Proof. The point $z_{0}$ is given implicitly by $d_{y} f\left(x, z_{0}\right)+d_{x} f\left(z_{0}, y\right)=0$. The nondegeneracy condition is

$$
\operatorname{det}\left[\frac{\partial}{\partial z_{i}}\left(\frac{\partial f}{\partial y_{j}}(x, z)+\frac{\partial f}{\partial x_{j}}(z, y)\right)\right] \neq 0
$$

By the implicit function theorem, $z_{0}=z_{0}(x, y)$ is smooth.

As for the second assertion, $f^{(2)}(x, y)$ is a generating function for $\varphi^{(2)}$ if and only if

$$
\varphi^{(2)}\left(x, d_{x} f^{(2)}\right)=\left(y,-d_{y} f^{(2)}\right)
$$

(assuming that, for each $\xi \in T_{x}^{*} X$, there is a unique $y \in X$ for which $d_{x} f^{(2)}=\xi$ ). Since $\varphi$ is generated by $f$, and $z_{0}$ is critical, we obtain

$$
\begin{aligned}
\varphi^{(2)}\left(x, d_{x} f^{(2)}(x, y)\right) & =\varphi\left(\varphi(x, \underbrace{d_{x} f^{(2)}(x, y)}_{=d_{x} f\left(x, z_{0}\right)})=\varphi\left(z_{0},-d_{y} f\left(x, z_{0}\right)\right)\right. \\
& =\varphi\left(z_{0}, d_{x} f\left(z_{0}, y\right)\right)=(y, \underbrace{-d_{y} f\left(z_{0}, y\right)}_{=-d_{y} f^{(2)}(x, y)}) .
\end{aligned}
$$

Exercise. What is a generating function for $\varphi^{(3)}$ ?

Hint: Suppose that the function

$$
\begin{aligned}
X \times X & \longrightarrow \mathbb{R} \\
(z, u) & \longmapsto f(x, z)+f(z, u)+f(u, y)
\end{aligned}
$$

has a unique critical point $\left(z_{0}, u_{0}\right)$, and that it is a nondegenerate critical point. Let $f^{(3)}(x, y)=f\left(x, z_{0}\right)+f\left(z_{0}, u_{0}\right)+f\left(u_{0}, y\right)$.

\subsection{Billiards}

Let $\chi: \mathbb{R} \rightarrow \mathbb{R}^{2}$ be a smooth plane curve which is 1-periodic, i.e., $\chi(s+1)=\chi(s)$, and parametrized by arc-length, i.e., $\left|\frac{d \chi}{d s}\right|=1$. Assume that the region $Y$ enclosed by $\chi$ is convex, i.e., for any $s \in \mathbb{R}$, the tangent line $\left\{\chi(s)+t \frac{d \chi}{d s} \mid t \in \mathbb{R}\right\}$ intersects $X:=\partial Y(=$ the image of $\chi)$ at only the point $\chi(s)$. 


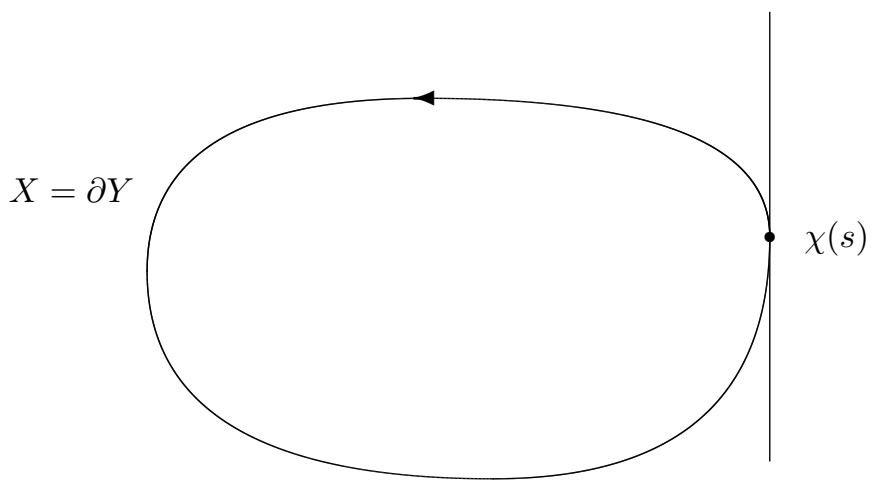

Suppose that we throw a ball into $Y$ rolling with constant velocity and bouncing off the boundary with the usual law of reflection. This determines a map

$$
\begin{aligned}
\varphi: \mathbb{R} / \mathbb{Z} \times(-1,1) & \longrightarrow \mathbb{R} / \mathbb{Z} \times(-1,1) \\
(x, v) & \longmapsto(y, w)
\end{aligned}
$$

by the rule

when the ball bounces off $\chi(x)$ with angle $\theta=\arccos v$, it will next collide with $\chi(y)$ and bounce off with angle $\nu=\arccos w$.

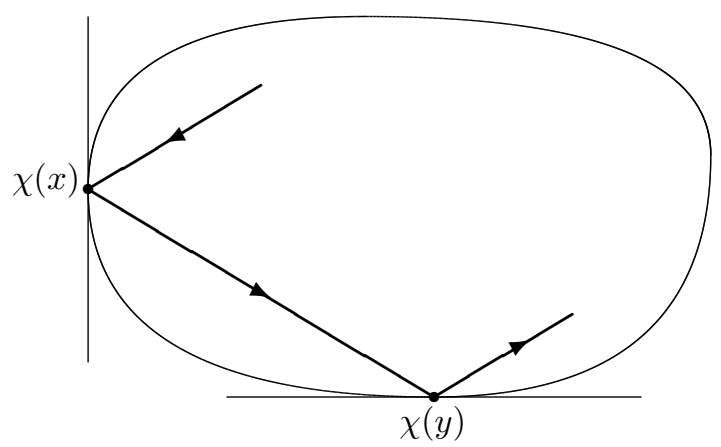

Let $f: \mathbb{R} / \mathbb{Z} \times \mathbb{R} / \mathbb{Z} \rightarrow \mathbb{R}$ be defined by $f(x, y)=-|\chi(x)-\chi(y)| ; f$ is smooth off the diagonal. Use $\chi$ to identify $\mathbb{R} / \mathbb{Z}$ with the image curve $X$.

Suppose that $\varphi(x, v)=(y, w)$, i.e., $(x, v)$ and $(y, w)$ are successive points on 
the orbit described by the ball. Then

$$
\left\{\begin{aligned}
\frac{d f}{d x} & =-\frac{x-y}{|x-y|} \text { projected onto } T_{x} X=v \\
\frac{d f}{d y} & =-\frac{y-x}{|x-y|} \text { projected onto } T_{y} X=-w
\end{aligned}\right.
$$

or, equivalently,

$$
\left\{\begin{aligned}
\frac{d}{d s} f(\chi(s), y) & =\frac{y-x}{|x-y|} \cdot \frac{d \chi}{d s}=\cos \theta=v \\
\frac{d}{d s} f(x, \chi(s)) & =\frac{x-y}{|x-y|} \cdot \frac{d \chi}{d s}=-\cos \nu=-w
\end{aligned}\right.
$$

We conclude that $f$ is a generating function for $\varphi$. Similar approaches work for higher dimensional billiards problems.

Periodic points are obtained by finding critical points of

$$
\begin{aligned}
& \underbrace{X \times \ldots \times X}_{N} \longrightarrow \mathbb{R}, \quad N>1 \\
& \left(x_{1}, \ldots, x_{N}\right) \longmapsto f\left(x_{1}, x_{2}\right)+f\left(x_{2}, x_{3}\right)+\ldots+f\left(x_{N-1}, x_{N}\right)+f\left(x_{N}, x_{1}\right) \\
& =\left|x_{1}-x_{2}\right|+\ldots+\left|x_{N-1}-x_{N}\right|+\left|x_{N}-x_{1}\right| \text {, }
\end{aligned}
$$

that is, by finding the $N$-sided (generalized) polygons inscribed in $X$ of critical perimeter.

Notice that

$$
\mathbb{R} / \mathbb{Z} \times(-1,1) \simeq\left\{(x, v)\left|x \in X, v \in T_{x} X,\right| v \mid<1\right\} \simeq A
$$

is the open unit tangent ball bundle of a circle $X$, that is, an open annulus $A$. The map $\varphi: A \rightarrow A$ is area-preserving.

\subsection{Poincaré Recurrence}

Theorem 5.3 (Poincaré Recurrence Theorem) Suppose that $\varphi: A \rightarrow A$ is an area-preserving diffeomorphism of a finite-area manifold $A$. Let $p \in A$, and let $\mathcal{U}$ be a neighborhood of $p$. Then there is $q \in \mathcal{U}$ and a positive integer $N$ such that $\varphi^{(N)}(q) \in \mathcal{U}$.

Proof. Let $\mathcal{U}_{0}=\mathcal{U}, \mathcal{U}_{1}=\varphi(\mathcal{U}), \mathcal{U}_{2}=\varphi^{(2)}(\mathcal{U}), \ldots$ If all of these sets were disjoint, then, since Area $\left(\mathcal{U}_{i}\right)=$ Area $(\mathcal{U})>0$ for all $i$, we would have

$$
\text { Area } A \geq \text { Area }\left(\mathcal{U}_{0} \cup \mathcal{U}_{1} \cup \mathcal{U}_{2} \cup \ldots\right)=\sum_{i} \text { Area }\left(\mathcal{U}_{i}\right)=\infty \text {. }
$$


To avoid this contradiction we must have $\varphi^{(k)}(\mathcal{U}) \cap \varphi^{(l)}(\mathcal{U}) \neq \emptyset$ for some $k>l$, which implies $\varphi^{(k-l)}(\mathcal{U}) \cap \mathcal{U} \neq \emptyset$.

Hence, eternal return applies to billiards...

Remark. Theorem 5.3 clearly generalizes to volume-preserving diffeomorphisms in higher dimensions.

Theorem 5.4 (Poincaré's Last Geometric Theorem) Suppose $\varphi: A \rightarrow A$ is an area-preserving diffeomorphism of the closed annulus $A=\mathbb{R} / \mathbb{Z} \times[-1,1]$ which preserves the two components of the boundary, and twists them in opposite directions. Then $\varphi$ has at least two fixed points.

This theorem was proved in 1913 by Birkhoff, and hence is also called the Poincaré-Birkhoff theorem. It has important applications to dynamical systems and celestial mechanics. The Arnold conjecture (1966) on the existence of fixed points for symplectomorphisms of compact manifolds (see Lecture 9) may be regarded as a generalization of the Poincaré-Birkhoff theorem. This conjecture has motivated a significant amount of recent research involving a more general notion of generating function; see, for instance, [34, 45]. 



\section{Part III}

\section{Local Forms}

Inspired by the elementary normal form in symplectic linear algebra (Theorem 1.1), we will go on to describe normal neighborhoods of a point (the Darboux theorem) and of a lagrangian submanifold (the Weinstein theorems), inside a symplectic manifold. The main tool is the Moser trick, explained in Lecture 7, which leads to the crucial Moser theorems and which is at the heart of many arguments in symplectic geometry.

In order to prove the normal forms, we need the (non-symplectic) ingredients discussed in Lecture 6; for more on these topics, see, for instance, $[18,55,96]$.

\section{Preparation for the Local Theory}

\subsection{Isotopies and Vector Fields}

Let $M$ be a manifold, and $\rho: M \times \mathbb{R} \rightarrow M$ a map, where we set $\rho_{t}(p):=\rho(p, t)$.

Definition 6.1 The map $\rho$ is an isotopy if each $\rho_{t}: M \rightarrow M$ is a diffeomorphism, and $\rho_{0}=\operatorname{id}_{M}$.

Given an isotopy $\rho$, we obtain a time-dependent vector field, that is, a family of vector fields $v_{t}, t \in \mathbb{R}$, which at $p \in M$ satisfy

$$
v_{t}(p)=\left.\frac{d}{d s} \rho_{s}(q)\right|_{s=t} \quad \text { where } \quad q=\rho_{t}^{-1}(p)
$$

i.e.,

$$
\frac{d \rho_{t}}{d t}=v_{t} \circ \rho_{t}
$$

Conversely, given a time-dependent vector field $v_{t}$, if $M$ is compact or if the $v_{t}$ 's are compactly supported, there exists an isotopy $\rho$ satisfying the previous ordinary differential equation.

Suppose that $M$ is compact. Then we have a one-to-one correspondence

$$
\begin{aligned}
\{\text { isotopies of } M\} & \stackrel{1-1}{\longleftrightarrow}\{\text { time-dependent vector fields on } M\} \\
\rho_{t}, t \in \mathbb{R} & \longleftrightarrow v_{t}, t \in \mathbb{R}
\end{aligned}
$$

Definition 6.2 When $v_{t}=v$ is independent of $t$, the associated isotopy is called the exponential map or the flow of $v$ and is denoted $\exp t v ;$ i.e., $\{\exp t v: M \rightarrow$ $M \mid t \in \mathbb{R}\}$ is the unique smooth family of diffeomorphisms satisfying

$$
\left.\exp t v\right|_{t=0}=\operatorname{id}_{M} \quad \text { and } \quad \frac{d}{d t}(\exp t v)(p)=v(\exp t v(p)) .
$$


Definition 6.3 The Lie derivative is the operator

$$
\mathcal{L}_{v}: \Omega^{k}(M) \longrightarrow \Omega^{k}(M) \quad \text { defined by } \quad \mathcal{L}_{v} \omega:=\left.\frac{d}{d t}(\exp t v)^{*} \omega\right|_{t=0} .
$$

When a vector field $v_{t}$ is time-dependent, its flow, that is, the corresponding isotopy $\rho$, still locally exists by Picard's theorem. More precisely, in the neighborhood of any point $p$ and for sufficiently small time $t$, there is a one-parameter family of local diffeomorphisms $\rho_{t}$ satisfying

$$
\frac{d \rho_{t}}{d t}=v_{t} \circ \rho_{t} \quad \text { and } \quad \rho_{0}=\mathrm{id} .
$$

Hence, we say that the Lie derivative by $v_{t}$ is

$$
\mathcal{L}_{v_{t}}: \Omega^{k}(M) \longrightarrow \Omega^{k}(M) \quad \text { defined by } \quad \mathcal{L}_{v_{t}} \omega:=\left.\frac{d}{d t}\left(\rho_{t}\right)^{*} \omega\right|_{t=0} .
$$

Exercise. Prove the Cartan magic formula,

$$
\mathcal{L}_{v} \omega=\imath_{v} d \omega+d \imath_{v} \omega,
$$

and the formula

$$
\frac{d}{d t} \rho_{t}^{*} \omega=\rho_{t}^{*} \mathcal{L}_{v_{t}} \omega
$$

where $\rho$ is the (local) isotopy generated by $v_{t}$. A good strategy for each formula is to follow the steps:

1. Check the formula for 0 -forms $\omega \in \Omega^{0}(M)=C^{\infty}(M)$.

2. Check that both sides commute with $d$.

3. Check that both sides are derivations of the algebra $\left(\Omega^{*}(M), \wedge\right)$. For instance, check that

$$
\mathcal{L}_{v}(\omega \wedge \alpha)=\left(\mathcal{L}_{v} \omega\right) \wedge \alpha+\omega \wedge\left(\mathcal{L}_{v} \alpha\right) .
$$

4. Notice that, if $\mathcal{U}$ is the domain of a coordinate system, then $\Omega^{\bullet}(\mathcal{U})$ is generated as an algebra by $\Omega^{0}(\mathcal{U})$ and $d \Omega^{0}(\mathcal{U})$, i.e., every element in $\Omega^{\bullet}(\mathcal{U})$ is a linear combination of wedge products of elements in $\Omega^{0}(\mathcal{U})$ and elements in $d \Omega^{0}(\mathcal{U})$.

We will need the following improved version of formula $(\star)$.

Proposition 6.4 For a smooth family $\omega_{t}, t \in \mathbb{R}$, of $d$-forms, we have

$$
\frac{d}{d t} \rho_{t}^{*} \omega_{t}=\rho_{t}^{*}\left(\mathcal{L}_{v_{t}} \omega_{t}+\frac{d \omega_{t}}{d t}\right)
$$


Proof. If $f(x, y)$ is a real function of two variables, by the chain rule we have

$$
\frac{d}{d t} f(t, t)=\left.\frac{d}{d x} f(x, t)\right|_{x=t}+\left.\frac{d}{d y} f(t, y)\right|_{y=t} .
$$

Therefore,

$$
\begin{aligned}
\frac{d}{d t} \rho_{t}^{*} \omega_{t} & =\underbrace{\left.\frac{d}{d x} \rho_{x}^{*} \omega_{t}\right|_{x=t}(\star)}_{\left.\rho_{x}^{*} \mathcal{L}_{v_{x}} \omega_{t}\right|_{x=t}}+\underbrace{\left.\frac{d}{d y} \rho_{t}^{*} \omega_{y}\right|_{y=t}}_{\left.\rho_{t}^{*} \frac{d \omega_{y}}{d y}\right|_{y=t}} \\
& =\rho_{t}^{*}\left(\mathcal{L}_{v_{t}} \omega_{t}+\frac{d \omega_{t}}{d t}\right) .
\end{aligned}
$$

\subsection{Tubular Neighborhood Theorem}

Let $M$ be an $n$-dimensional manifold, and let $X$ be a $k$-dimensional submanifold where $k<n$ and with inclusion map

$$
i: X \hookrightarrow M .
$$

At each $x \in X$, the tangent space to $X$ is viewed as a subspace of the tangent space to $M$ via the linear inclusion $d i_{x}: T_{x} X \hookrightarrow T_{x} M$, where we denote $x=i(x)$. The quotient $N_{x} X:=T_{x} M / T_{x} X$ is an $(n-k)$-dimensional vector space, known as the normal space to $X$ at $x$. The normal bundle of $X$ is

$$
N X=\left\{(x, v) \mid x \in X, v \in N_{x} X\right\} .
$$

The set $N X$ has the structure of a vector bundle over $X$ of rank $n-k$ under the natural projection, hence as a manifold $N X$ is $n$-dimensional. The zero section of $N X$,

$$
i_{0}: X \hookrightarrow N X, \quad x \mapsto(x, 0),
$$

embeds $X$ as a closed submanifold of $N X$. A neighborhood $\mathcal{U}_{0}$ of the zero section $X$ in $N X$ is called convex if the intersection $\mathcal{U}_{0} \cap N_{x} X$ with each fiber is convex.

Theorem 6.5 (Tubular Neighborhood Theorem) There exist a convex neighborhood $\mathcal{U}_{0}$ of $X$ in $N X$, a neighborhood $\mathcal{U}$ of $X$ in $M$, and a diffeomorphism $\varphi: \mathcal{U}_{0} \rightarrow \mathcal{U}$ such that

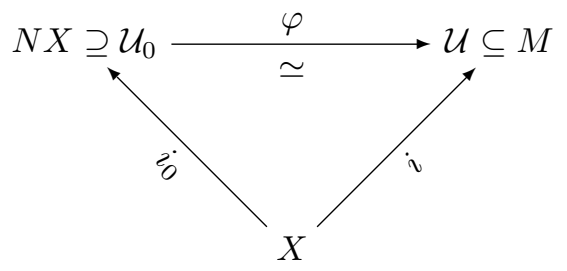

commutes. 


\section{Outline of the proof.}

- Case of $M=\mathbb{R}^{n}$, and $X$ is a compact submanifold of $\mathbb{R}^{n}$.

\section{Theorem 6.6 ( $\varepsilon$-Neighborhood Theorem)}

Let $\mathcal{U}^{\varepsilon}=\left\{p \in \mathbb{R}^{n}:|p-q|<\varepsilon\right.$ for some $\left.q \in X\right\}$ be the set of points at a distance less than $\varepsilon$ from $X$. Then, for $\varepsilon$ sufficiently small, each $p \in \mathcal{U}^{\varepsilon}$ has a unique nearest point $q \in X$ (i.e., a unique $q \in X$ minimizing $|q-x|$ ).

Moreover, setting $q=\pi(p)$, the map $\mathcal{U}^{\varepsilon} \stackrel{\pi}{\rightarrow} X$ is a (smooth) submersion with the property that, for all $p \in \mathcal{U}^{\varepsilon}$, the line segment $(1-t) p+t q, 0 \leq t \leq 1$, is in $\mathcal{U}^{\varepsilon}$.

The proof is part of Homework 5. Here are some hints.

At any $x \in X$, the normal space $N_{x} X$ may be regarded as an $(n-k)$ dimensional subspace of $\mathbb{R}^{n}$, namely the orthogonal complement in $\mathbb{R}^{n}$ of the tangent space to $X$ at $x$ :

$$
N_{x} X \simeq\left\{v \in \mathbb{R}^{n}: v \perp w, \text { for all } w \in T_{x} X\right\} .
$$

We define the following open neighborhood of $X$ in $N X$ :

$$
N X^{\varepsilon}=\{(x, v) \in N X:|v|<\varepsilon\} .
$$

Let

$$
\begin{aligned}
\exp : \quad & \longrightarrow \mathbb{R}^{n} \\
(x, v) & \longmapsto x+v .
\end{aligned}
$$

Restricted to the zero section, exp is the identity map on $X$.

Prove that, for $\varepsilon$ sufficiently small, exp maps $N X^{\varepsilon}$ diffeomorphically onto $\mathcal{U}^{\varepsilon}$, and show also that the diagram

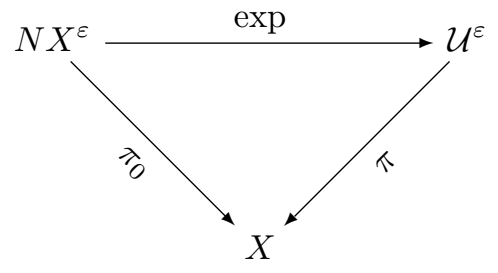

commutes.

- Case where $X$ is a compact submanifold of an arbitrary manifold $M$.

Put a riemannian metric $g$ on $M$, and let $d(p, q)$ be the riemannian distance between $p, q \in M$. The $\varepsilon$-neighborhood of a compact submanifold $X$ is

$$
\mathcal{U}^{\varepsilon}=\{p \in M \mid d(p, q)<\varepsilon \text { for some } q \in X\} .
$$

Prove the $\varepsilon$-neighborhood theorem in this setting: for $\varepsilon$ small enough, the following assertions hold. 
- Any $p \in \mathcal{U}^{\varepsilon}$ has a unique point $q \in X$ with minimal $d(p, q)$. Set $q=\pi(p)$.

- The map $\mathcal{U}^{\varepsilon} \stackrel{\pi}{\rightarrow} X$ is a submersion and, for all $p \in \mathcal{U}^{\varepsilon}$, there is a unique geodesic curve $\gamma$ joining $p$ to $q=\pi(p)$.

- The normal space to $X$ at $x \in X$ is naturally identified with a subspace of $T_{x} M$ :

$$
N_{x} X \simeq\left\{v \in T_{x} M \mid g_{x}(v, w)=0, \text { for any } w \in T_{x} X\right\} .
$$

Let $N X^{\varepsilon}=\left\{(x, v) \in N X \mid \sqrt{g_{x}(v, v)}<\varepsilon\right\}$.

- Define exp : $N X^{\varepsilon} \rightarrow M$ by $\exp (x, v)=\gamma(1)$, where $\gamma:[0,1] \rightarrow M$ is the geodesic with $\gamma(0)=x$ and $\frac{d \gamma}{d t}(0)=v$. Then exp maps $N X^{\varepsilon}$ diffeomorphically to $\mathcal{U}^{\varepsilon}$.

- General case.

When $X$ is not compact, adapt the previous argument by replacing $\varepsilon$ by an appropriate continuous function $\varepsilon: X \rightarrow \mathbb{R}^{+}$which tends to zero fast enough as $x$ tends to infinity.

Restricting to the subset $\mathcal{U}^{0} \subseteq N X$ from the tubular neighborhood theorem, we obtain a submersion $\mathcal{U}_{0} \stackrel{\pi_{0}}{\longrightarrow} X$ with all fibers $\pi_{0}^{-1}(x)$ convex. We can carry this fibration to $\mathcal{U}$ by setting $\pi=\pi_{0} \circ \varphi^{-1}$ :

$$
\begin{gathered}
\mathcal{U}_{0} \\
\pi_{0} \downarrow \\
X
\end{gathered} \quad \subseteq N X \text { is a fibration } \Longrightarrow \quad \begin{array}{r}
\mathcal{U} \\
\pi \downarrow \\
X
\end{array}
$$

This is called the tubular neighborhood fibration.

\subsection{Homotopy Formula}

Let $\mathcal{U}$ be a tubular neighborhood of a submanifold $X$ in $M$. The restriction $i^{*}$ : $H_{\text {deRham }}^{d}(\mathcal{U}) \rightarrow H_{\text {deRham }}^{d}(X)$ by the inclusion map is surjective. As a corollary of the tubular neighborhood fibration, $i^{*}$ is also injective: this follows from the homotopy-invariance of de Rham cohomology.

Corollary 6.7 For any degree $\ell, H_{\text {deRham }}^{\ell}(\mathcal{U}) \simeq H_{\text {deRham }}^{\ell}(X)$.

At the level of forms, this means that, if $\omega$ is a closed $\ell$-form on $\mathcal{U}$ and $i^{*} \omega$ is exact on $X$, then $\omega$ is exact. We will need the following related result.

Proposition 6.8 If a closed $\ell$-form $\omega$ on $\mathcal{U}$ has restriction $i^{*} \omega=0$, then $\omega$ is exact, i.e., $\omega=d \mu$ for some $\mu \in \Omega^{d-1}(\mathcal{U})$. Moreover, we can choose $\mu$ such that $\mu_{x}=0$ at all $x \in X$. 
Proof. Via $\varphi: \mathcal{U}_{0} \stackrel{\simeq}{\longrightarrow} \mathcal{U}$, it is equivalent to work over $\mathcal{U}_{0}$. Define for every $0 \leq t \leq 1$ a map

$$
\begin{aligned}
\rho_{t}: \quad \mathcal{U}_{0} & \longrightarrow \mathcal{U}_{0} \\
(x, v) & \longmapsto(x, t v) .
\end{aligned}
$$

This is well-defined since $\mathcal{U}_{0}$ is convex. The map $\rho_{1}$ is the identity, $\rho_{0}=i_{0} \circ \pi_{0}$, and each $\rho_{t}$ fixes $X$, that is, $\rho_{t} \circ i_{0}=i_{0}$. We hence say that the family $\left\{\rho_{t} \mid 0 \leq t \leq 1\right\}$ is a homotopy from $i_{0} \circ \pi_{0}$ to the identity fixing $X$. The map $\pi_{0}: \mathcal{U}_{0} \rightarrow X$ is called a retraction because $\pi_{0} \circ i_{0}$ is the identity. The submanifold $X$ is then called a deformation retract of $\mathcal{U}$.

A (de Rham) homotopy operator between $\rho_{0}=i_{0} \circ \pi_{0}$ and $\rho_{1}=$ id is a linear map

$$
Q: \Omega^{d}\left(\mathcal{U}_{0}\right) \longrightarrow \Omega^{d-1}\left(\mathcal{U}_{0}\right)
$$

satisfying the homotopy formula

$$
\mathrm{Id}-\left(i_{0} \circ \pi_{0}\right)^{*}=d Q+Q d .
$$

When $d \omega=0$ and $i_{0}^{*} \omega=0$, the operator $Q$ gives $\omega=d Q \omega$, so that we can take $\mu=Q \omega$. A concrete operator $Q$ is given by the formula:

$$
Q \omega=\int_{0}^{1} \rho_{t}^{*}\left(\imath_{v_{t}} \omega\right) d t
$$

where $v_{t}$, at the point $q=\rho_{t}(p)$, is the vector tangent to the curve $\rho_{s}(p)$ at $s=t$. The proof that $Q$ satisfies the homotopy formula is below.

In our case, for $x \in X, \rho_{t}(x)=x$ (all $t$ ) is the constant curve, so $v_{t}$ vanishes at all $x$ for all $t$, hence $\mu_{x}=0$.

To check that $Q$ above satisfies the homotopy formula, we compute

$$
\begin{aligned}
Q d \omega+d Q \omega & =\int_{0}^{1} \rho_{t}^{*}\left(\imath_{v_{t}} d \omega\right) d t+d \int_{0}^{1} \rho_{t}^{*}\left(\imath_{v_{t}} \omega\right) d t \\
& =\int_{0}^{1} \rho_{t}^{*}(\underbrace{\left(l_{v_{t}} d \omega+d \imath_{v_{t}} \omega\right.}_{\mathcal{L}_{v_{t}} \omega}) d t
\end{aligned}
$$

where $\mathcal{L}_{v}$ denotes the Lie derivative along $v$ (reviewed in the next section), and we used the Cartan magic formula: $\mathcal{L}_{v} \omega=\imath_{v} d \omega+d \imath_{v} \omega$. The result now follows from

$$
\frac{d}{d t} \rho_{t}^{*} \omega=\rho_{t}^{*} \mathcal{L}_{v_{t}} \omega
$$

and from the fundamental theorem of calculus:

$$
Q d \omega+d Q \omega=\int_{0}^{1} \frac{d}{d t} \rho_{t}^{*} \omega d t=\rho_{1}^{*} \omega-\rho_{0}^{*} \omega .
$$




\section{Homework 5: Tubular Neighborhoods in $\mathbb{R}^{n}$}

1. Let $X$ be a $k$-dimensional submanifold of an $n$-dimensional manifold $M$. Let $x$ be a point in $X$. The normal space to $X$ at $x$ is the quotient space

$$
N_{x} X=T_{x} M / T_{x} X,
$$

and the normal bundle of $X$ in $M$ is the vector bundle $N X$ over $X$ whose fiber at $x$ is $N_{x} X$.

(a) Prove that $N X$ is indeed a vector bundle.

(b) If $M$ is $\mathbb{R}^{n}$, show that $N_{x} X$ can be identified with the usual "normal space" to $X$ in $\mathbb{R}^{n}$, that is, the orthogonal complement in $\mathbb{R}^{n}$ of the tangent space to $X$ at $x$.

2. Let $X$ be a $k$-dimensional compact submanifold of $\mathbb{R}^{n}$. Prove the tubular neighborhood theorem in the following form.

(a) Given $\varepsilon>0$ let $\mathcal{U}_{\varepsilon}$ be the set of all points in $\mathbb{R}^{n}$ which are at a distance less than $\varepsilon$ from $X$. Show that, for $\varepsilon$ sufficiently small, every point $p \in \mathcal{U}_{\varepsilon}$ has a unique nearest point $\pi(p) \in X$.

(b) Let $\pi: \mathcal{U}_{\varepsilon} \rightarrow X$ be the map defined in (a) for $\varepsilon$ sufficiently small. Show that, if $p \in \mathcal{U}_{\varepsilon}$, then the line segment $(1-t) \cdot p+t \cdot \pi(p), 0 \leq t \leq 1$, joining $p$ to $\pi(p)$ lies in $\mathcal{U}_{\varepsilon}$.

(c) Let $N X_{\varepsilon}=\{(x, v) \in N X$ such that $|v|<\varepsilon\}$. Let $\exp : N X \rightarrow \mathbb{R}^{n}$ be the map $(x, v) \mapsto x+v$, and let $\nu: N X_{\varepsilon} \rightarrow X$ be the map $(x, v) \mapsto x$. Show that, for $\varepsilon$ sufficiently small, exp maps $N X_{\varepsilon}$ diffeomorphically onto $\mathcal{U}_{\varepsilon}$, and show also that the following diagram commutes:

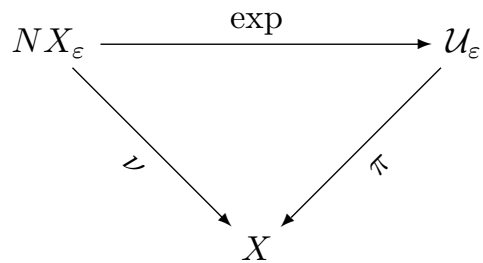

3. Suppose that the manifold $X$ in the previous exercise is not compact.

Prove that the assertion about exp is still true provided we replace $\varepsilon$ by a continuous function

$$
\varepsilon: X \rightarrow \mathbb{R}^{+}
$$

which tends to zero fast enough as $x$ tends to infinity. 


\section{Moser Theorems}

\subsection{Notions of Equivalence for Symplectic Structures}

Let $M$ be a $2 n$-dimensional manifold with two symplectic forms $\omega_{0}$ and $\omega_{1}$, so that $\left(M, \omega_{0}\right)$ and $\left(M, \omega_{1}\right)$ are two symplectic manifolds.

Definition 7.1 We say that

- $\left(M, \omega_{0}\right)$ and $\left(M, \omega_{1}\right)$ are symplectomorphic if there is a diffeomorphism $\varphi: M \rightarrow M$ with $\varphi^{*} \omega_{1}=\omega_{0}$;

- $\left(M, \omega_{0}\right)$ and $\left(M, \omega_{1}\right)$ are strongly isotopic if there is an isotopy $\rho_{t}: M \rightarrow M$ such that $\rho_{1}^{*} \omega_{1}=\omega_{0}$;

- $\left(M, \omega_{0}\right)$ and $\left(M, \omega_{1}\right)$ are deformation-equivalent if there is a smooth family $\omega_{t}$ of symplectic forms joining $\omega_{0}$ to $\omega_{1}$;

- $\left(M, \omega_{0}\right)$ and $\left(M, \omega_{1}\right)$ are isotopic if they are deformation-equivalent with $\left[\omega_{t}\right]$ independent of $t$.

Clearly, we have

$$
\begin{array}{ccc}
\text { strongly isotopic } & \Longrightarrow \text { symplectomorphic, and } \\
\text { isotopic } & \Longrightarrow \text { deformation-equivalent . }
\end{array}
$$

We also have

$$
\text { strongly isotopic } \Longrightarrow \text { isotopic }
$$

because, if $\rho_{t}: M \rightarrow M$ is an isotopy such that $\rho_{1}^{*} \omega_{1}=\omega_{0}$, then the set $\omega_{t}:=\rho_{t}^{*} \omega_{1}$ is a smooth family of symplectic forms joining $\omega_{1}$ to $\omega_{0}$ and $\left[\omega_{t}\right]=\left[\omega_{1}\right], \forall t$, by the homotopy invariance of de Rham cohomology. As we will see below, the Moser theorem states that, on a compact manifold,

$$
\text { isotopic } \Longrightarrow \text { strongly isotopic . }
$$

\subsection{Moser Trick}

Problem. Given a $2 n$-dimensional manifold $M$, a $k$-dimensional submanifold $X$, neighborhoods $\mathcal{U}_{0}, \mathcal{U}_{1}$ of $X$, and symplectic forms $\omega_{0}, \omega_{1}$ on $\mathcal{U}_{0}, \mathcal{U}_{1}$, does there exist a symplectomorphism preserving $X$ ? More precisely, does there exist a diffeomorphism $\varphi: \mathcal{U}_{0} \rightarrow \mathcal{U}_{1}$ with $\varphi^{*} \omega_{1}=\omega_{0}$ and $\varphi(X)=X$ ?

At the two extremes, we have:
Case $X=$ point:
Darboux theorem - see Lecture 8.
Case $X=M$ :
Moser theorem - discussed here:

Let $M$ be a compact manifold with symplectic forms $\omega_{0}$ and $\omega_{1}$. 
- Are $\left(M, \omega_{0}\right)$ and $\left(M, \omega_{1}\right)$ symplectomorphic?

I.e., does there exist a diffeomorphism $\varphi: M \rightarrow M$ such that $\varphi_{1}^{*} \omega_{0}=\omega_{1}$ ?

Moser asked whether we can find such an $\varphi$ which is homotopic to $\operatorname{id}_{M}$. A necessary condition is $\left[\omega_{0}\right]=\left[\omega_{1}\right] \in H^{2}(M ; \mathbb{R})$ because: if $\varphi \sim \operatorname{id}_{M}$, then, by the homotopy formula, there exists a homotopy operator $Q$ such that

$$
\begin{array}{cc} 
& \operatorname{id}_{M}^{*} \omega_{1}-\varphi^{*} \omega_{1}=d Q \omega_{1}+Q \underbrace{d \omega_{1}}_{0} \\
\Longrightarrow & \omega_{1}=\varphi^{*} \omega_{1}+d\left(Q \omega_{1}\right) \\
\Longrightarrow & {\left[\omega_{1}\right]=\left[\varphi^{*} \omega_{1}\right]=\left[\omega_{0}\right] .}
\end{array}
$$

- If $\left[\omega_{0}\right]=\left[\omega_{1}\right]$, does there exist a diffeomorphism $\varphi$ homotopic to $\operatorname{id}_{M}$ such that $\varphi^{*} \omega_{1}=\omega_{0}$ ?

Moser [87] proved that the answer is yes, with a further hypothesis as in Theorem 7.2. McDuff showed that, in general, the answer is no; for a counterexample, see Example 7.23 in [83].

Theorem 7.2 (Moser Theorem - Version I) Suppose that $M$ is compact, $\left[\omega_{0}\right]=\left[\omega_{1}\right]$ and that the 2-form $\omega_{t}=(1-t) \omega_{0}+t \omega_{1}$ is symplectic for each $t \in[0,1]$. Then there exists an isotopy $\rho: M \times \mathbb{R} \rightarrow M$ such that $\rho_{t}^{*} \omega_{t}=\omega_{0}$ for all $t \in[0,1]$.

In particular, $\varphi=\rho_{1}: M \stackrel{\simeq}{\longrightarrow} M$, satisfies $\varphi^{*} \omega_{1}=\omega_{0}$.

The following argument, due to Moser, is extremely useful; it is known as the Moser trick.

Proof. Suppose that there exists an isotopy $\rho: M \times \mathbb{R} \rightarrow M$ such that $\rho_{t}^{*} \omega_{t}=\omega_{0}$, $0 \leq t \leq 1$. Let

$$
v_{t}=\frac{d \rho_{t}}{d t} \circ \rho_{t}^{-1}, \quad t \in \mathbb{R} .
$$

Then

$$
\begin{aligned}
& 0=\frac{d}{d t}\left(\rho_{t}^{*} \omega_{t}\right)=\rho_{t}^{*}\left(\mathcal{L}_{v_{t}} \omega_{t}+\frac{d \omega_{t}}{d t}\right) \\
& \Longleftrightarrow \quad \mathcal{L}_{v_{t}} \omega_{t}+\frac{d \omega_{t}}{d t}=0 .
\end{aligned}
$$

Suppose conversely that we can find a smooth time-dependent vector field $v_{t}$, $t \in \mathbb{R}$, such that $(\star)$ holds for $0 \leq t \leq 1$. Since $M$ is compact, we can integrate $v_{t}$ to an isotopy $\rho: M \times \mathbb{R} \rightarrow M$ with

$$
\frac{d}{d t}\left(\rho_{t}^{*} \omega_{t}\right)=0 \quad \Longrightarrow \quad \rho_{t}^{*} \omega_{t}=\rho_{0}^{*} \omega_{0}=\omega_{0} .
$$

So everything boils down to solving $(\star)$ for $v_{t}$.

First, from $\omega_{t}=(1-t) \omega_{0}+t \omega_{1}$, we conclude that

$$
\frac{d \omega_{t}}{d t}=\omega_{1}-\omega_{0}
$$


Second, since $\left[\omega_{0}\right]=\left[\omega_{1}\right]$, there exists a 1 -form $\mu$ such that

$$
\omega_{1}-\omega_{0}=d \mu \text {. }
$$

Third, by the Cartan magic formula, we have

$$
\mathcal{L}_{v_{t}} \omega_{t}=d \imath_{v_{t}} \omega_{t}+\imath_{v_{t}} \underbrace{d \omega_{t}}_{0} .
$$

Putting everything together, we must find $v_{t}$ such that

$$
d v_{v_{t}} \omega_{t}+d \mu=0 .
$$

It is sufficient to solve $\imath_{v_{t}} \omega_{t}+\mu=0$. By the nondegeneracy of $\omega_{t}$, we can solve this pointwise, to obtain a unique (smooth) $v_{t}$.

Theorem 7.3 (Moser Theorem - Version II) Let $M$ be a compact manifold with symplectic forms $\omega_{0}$ and $\omega_{1}$. Suppose that $\omega_{t}, 0 \leq t \leq 1$, is a smooth family of closed 2-forms joining $\omega_{0}$ to $\omega_{1}$ and satisfying:

(1) cohomology assumption: $\left[\omega_{t}\right]$ is independent of $t$, i.e., $\frac{d}{d t}\left[\omega_{t}\right]=\left[\frac{d}{d t} \omega_{t}\right]=0$,

(2) nondegeneracy assumption: $\omega_{t}$ is nondegenerate for $0 \leq t \leq 1$.

Then there exists an isotopy $\rho: M \times \mathbb{R} \rightarrow M$ such that $\rho_{t}^{*} \omega_{t}=\omega_{0}, 0 \leq t \leq 1$.

Proof. (Moser trick) We have the following implications from the hypotheses:

(1) $\Longrightarrow \exists$ family of 1 -forms $\mu_{t}$ such that

$$
\frac{d \omega_{t}}{d t}=d \mu_{t}, \quad 0 \leq t \leq 1
$$

We can indeed find a smooth family of 1 -forms $\mu_{t}$ such that $\frac{d \omega_{t}}{d t}=d \mu_{t}$. The argument involves the Poincare lemma for compactly-supported forms, together with the Mayer-Vietoris sequence in order to use induction on the number of charts in a good cover of $M$. For a sketch of the argument, see page 95 in [83].

(2) $\Longrightarrow \exists$ unique family of vector fields $v_{t}$ such that

$$
\imath_{v_{t}} \omega_{t}+\mu_{t}=0 \quad \text { (Moser equation) }
$$

Extend $v_{t}$ to all $t \in \mathbb{R}$. Let $\rho$ be the isotopy generated by $v_{t}$ ( $\rho$ exists by compactness of $M$ ). Then we indeed have

$$
\frac{d}{d t}\left(\rho_{t}^{*} \omega_{t}\right)=\rho_{t}^{*}\left(\mathcal{L}_{v_{t}} \omega_{t}+\frac{d \omega_{t}}{d t}\right)=\rho_{t}^{*}\left(d v_{v_{t}} \omega_{t}+d \mu_{t}\right)=0 .
$$


The compactness of $M$ was used to be able to integrate $v_{t}$ for all $t \in \mathbb{R}$. If $M$ is not compact, we need to check the existence of a solution $\rho_{t}$ for the differential equation $\frac{d \rho_{t}}{d t}=v_{t} \circ \rho_{t}$ for $0 \leq t \leq 1$.

Picture. Fix $c \in H^{2}(M)$. Define $S_{c}=\{$ symplectic forms $\omega$ in $M$ with $[\omega]=c\}$. The Moser theorem implies that, on a compact manifold, all symplectic forms on the same path-connected component of $S_{c}$ are symplectomorphic.

\subsection{Moser Relative Theorem}

Theorem 7.4 (Moser Theorem - Relative Version) Let $M$ be a manifold, $X$ a compact submanifold of $M, i: X \hookrightarrow M$ the inclusion map, $\omega_{0}$ and $\omega_{1}$ symplectic forms in $M$.

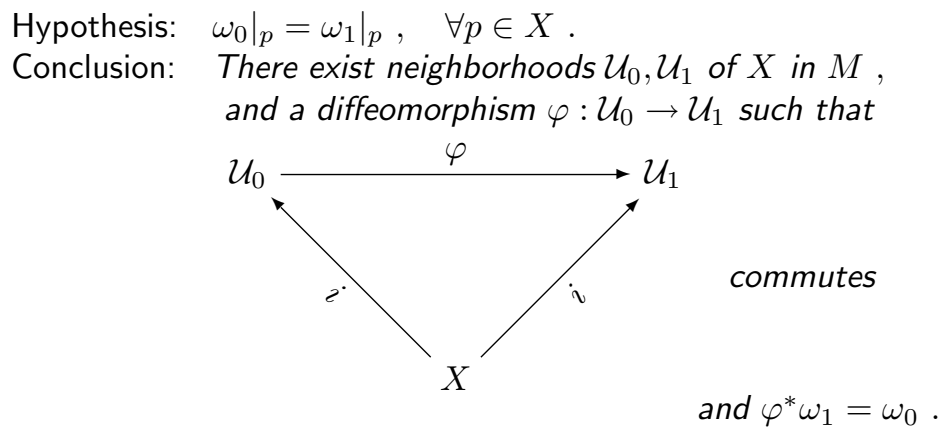

\section{Proof.}

1. Pick a tubular neighborhood $\mathcal{U}_{0}$ of $X$. The 2-form $\omega_{1}-\omega_{0}$ is closed on $\mathcal{U}_{0}$, and $\left(\omega_{1}-\omega_{0}\right)_{p}=0$ at all $p \in X$. By the homotopy formula on the tubular neighborhood, there exists a 1 -form $\mu$ on $\mathcal{U}_{0}$ such that $\omega_{1}-\omega_{0}=d \mu$ and $\mu_{p}=0$ at all $p \in X$.

2. Consider the family $\omega_{t}=(1-t) \omega_{0}+t \omega_{1}=\omega_{0}+t d \mu$ of closed 2-forms on $\mathcal{U}_{0}$. Shrinking $\mathcal{U}_{0}$ if necessary, we can assume that $\omega_{t}$ is symplectic for $0 \leq t \leq 1$.

3. Solve the Moser equation: $\imath_{v_{t}} \omega_{t}=-\mu$. Notice that $v_{t}=0$ on $X$.

4. Integrate $v_{t}$. Shrinking $\mathcal{U}_{0}$ again if necessary, there exists an isotopy $\rho$ : $\mathcal{U}_{0} \times[0,1] \rightarrow M$ with $\rho_{t}^{*} \omega_{t}=\omega_{0}$, for all $t \in[0,1]$. Since $\left.v_{t}\right|_{X}=0$, we have $\left.\rho_{t}\right|_{X}=\operatorname{id}_{X}$.

Set $\varphi=\rho_{1}, \mathcal{U}_{1}=\rho_{1}\left(\mathcal{U}_{0}\right)$.

Exercise. Prove the Darboux theorem. (Hint: apply the relative version of the Moser theorem to $X=\{p\}$, as in the next lecture.) 


\section{Darboux-Moser-Weinstein Theory}

\subsection{Darboux Theorem}

Theorem 8.1 (Darboux) Let $(M, \omega)$ be a symplectic manifold, and let $p$ be any point in $M$. Then we can find a coordinate system $\left(\mathcal{U}, x_{1}, \ldots, x_{n}, y_{1}, \ldots y_{n}\right)$ centered at $p$ such that on $\mathcal{U}$

$$
\omega=\sum_{i=1}^{n} d x_{i} \wedge d y_{i}
$$

As a consequence of Theorem 8.1, if we prove for $\left(\mathbb{R}^{2 n}, \sum d x_{i} \wedge d y_{i}\right)$ a local assertion which is invariant under symplectomorphisms, then that assertion holds for any symplectic manifold.

Proof. Apply the Moser relative theorem (Theorem 7.4) to $X=\{p\}$ :

Use any symplectic basis for $T_{p} M$ to construct coordinates $\left(x_{1}^{\prime}, \ldots, x_{n}^{\prime}, y_{1}^{\prime}, \ldots y_{n}^{\prime}\right)$ centered at $p$ and valid on some neighborhood $\mathcal{U}^{\prime}$, so that

$$
\omega_{p}=\left.\sum d x_{i}^{\prime} \wedge d y_{i}^{\prime}\right|_{p} .
$$

There are two symplectic forms on $\mathcal{U}^{\prime}$ : the given $\omega_{0}=\omega$ and $\omega_{1}=\sum d x_{i}^{\prime} \wedge d y_{i}^{\prime}$. By the Moser theorem, there are neighborhoods $\mathcal{U}_{0}$ and $\mathcal{U}_{1}$ of $p$, and a diffeomorphism $\varphi: \mathcal{U}_{0} \rightarrow \mathcal{U}_{1}$ such that

$$
\varphi(p)=p \quad \text { and } \quad \varphi^{*}\left(\sum d x_{i}^{\prime} \wedge d y_{i}^{\prime}\right)=\omega .
$$

Since $\varphi^{*}\left(\sum d x_{i}^{\prime} \wedge d y_{i}^{\prime}\right)=\sum d\left(x_{i}^{\prime} \circ \varphi\right) \wedge d\left(y_{i}^{\prime} \circ \varphi\right)$, we only need to set new coordinates $x_{i}=x_{i}^{\prime} \circ \varphi$ and $y_{i}=y_{i}^{\prime} \circ \varphi$.

If in the Moser relative theorem (Theorem 7.4) we assume instead

$$
\begin{array}{ll}
\text { Hypothesis: } & X \text { is an } n \text {-dimensional submanifold with } \\
& i^{*} \omega_{0}=i^{*} \omega_{1}=0 \text { where } i: X \hookrightarrow M \text { is inclusion, i.e., } \\
& X \text { is a submanifold lagrangian for } \omega_{0} \text { and } \omega_{1},
\end{array}
$$

then Weinstein [104] proved that the conclusion still holds. We need some algebra for the Weinstein theorem.

\subsection{Lagrangian Subspaces}

Suppose that $U, W$ are $n$-dimensional vector spaces, and $\Omega: U \times W \rightarrow \mathbb{R}$ is a bilinear pairing; the map $\Omega$ gives rise to a linear map $\widetilde{\Omega}: U \rightarrow W^{*}, \widetilde{\Omega}(u)=\Omega(u, \cdot)$. Then $\Omega$ is nondegenerate if and only if $\widetilde{\Omega}$ is bijective. 
Proposition 8.2 Suppose that $(V, \Omega)$ is a $2 n$-dimensional symplectic vector space. Let $U$ be a lagrangian subspace of $(V, \Omega)$ (i.e., $\left.\Omega\right|_{U \times U}=0$ and $U$ is $n$-dimensional). Let $W$ be any vector space complement to $U$, not necessarily lagrangian.

Then from $W$ we can canonically build a lagrangian complement to $U$.

Proof. The pairing $\Omega$ gives a nondegenerate pairing $U \times W \stackrel{\Omega^{\prime}}{\rightarrow} \mathbb{R}$. Therefore, $\widetilde{\Omega^{\prime}}: U \rightarrow W^{*}$ is bijective. We look for a lagrangian complement to $U$ of the form

$$
W^{\prime}=\{w+A w \mid w \in W\},
$$

the map $A: W \rightarrow U$ being linear. For $W^{\prime}$ to be lagrangian we need

$$
\begin{aligned}
& \forall w_{1}, w_{2} \in W, \quad \Omega\left(w_{1}+A w_{1}, w_{2}+A w_{2}\right)=0 \\
& \Longrightarrow \Omega\left(w_{1}, w_{2}\right)+\Omega\left(w_{1}, A w_{2}\right)+\Omega\left(A w_{1}, w_{2}\right)+\underbrace{\Omega(\underbrace{A w_{1}, A w_{2}}_{\in U})}_{0}=0 \\
& \Longrightarrow \Omega\left(w_{1}, w_{2}\right)=\Omega\left(A w_{2}, w_{1}\right)-\Omega\left(A w_{1}, w_{2}\right) \\
& =\widetilde{\Omega}^{\prime}\left(A w_{2}\right)\left(w_{1}\right)-\widetilde{\Omega}^{\prime}\left(A w_{1}\right)\left(w_{2}\right) .
\end{aligned}
$$

Let $A^{\prime}=\widetilde{\Omega}^{\prime} \circ A: W \rightarrow W^{*}$, and look for $A^{\prime}$ such that

$$
\forall w_{1}, w_{2} \in W, \quad \Omega\left(w_{1}, w_{2}\right)=A^{\prime}\left(w_{2}\right)\left(w_{1}\right)-A^{\prime}\left(w_{1}\right)\left(w_{2}\right) .
$$

The canonical choice is $A^{\prime}(w)=-\frac{1}{2} \Omega(w, \cdot)$. Then set $A=\left(\widetilde{\Omega}^{\prime}\right)^{-1} \circ A^{\prime}$.

Proposition 8.3 Let $V$ be a $2 n$-dimensional vector space, let $\Omega_{0}$ and $\Omega_{1}$ be symplectic forms in $V$, let $U$ be a subspace of $V$ lagrangian for $\Omega_{0}$ and $\Omega_{1}$, and let $W$ be any complement to $U$ in $V$. Then from $W$ we can canonically construct a linear isomorphism $L: V \stackrel{\simeq}{\rightarrow} V$ such that $\left.L\right|_{U}=\operatorname{Id}_{U}$ and $L^{*} \Omega_{1}=\Omega_{0}$.

Proof. From $W$ we canonically obtain complements $W_{0}$ and $W_{1}$ to $U$ in $V$ such that $W_{0}$ is lagrangian for $\Omega_{0}$ and $W_{1}$ is lagrangian for $\Omega_{1}$. The nondegenerate bilinear pairings

$$
\begin{array}{lll}
W_{0} \times U \stackrel{\Omega_{0}}{\longrightarrow} \mathbb{R} & \text { give isomorphisms } & \widetilde{\Omega}_{0}: W_{0} \stackrel{\simeq}{\longrightarrow} U^{*} \\
W_{1} \times U \stackrel{\Omega_{1}}{\longrightarrow} \mathbb{R} & \widetilde{\Omega}_{1}: W_{1} \stackrel{\simeq}{\longrightarrow} U^{*} .
\end{array}
$$

Consider the diagram

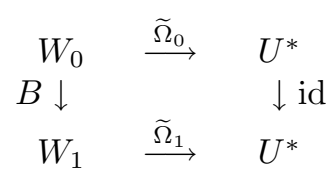

where the linear map $B$ satisfies $\widetilde{\Omega}_{1} \circ B=\widetilde{\Omega}_{0}$, i.e., $\Omega_{0}\left(w_{0}, u\right)=\Omega_{1}\left(B w_{0}, u\right)$, $\forall w_{0} \in W_{0}, \forall u \in U$. Extend $B$ to the rest of $V$ by setting it to be the identity on $U$ :

$$
L:=\operatorname{Id}_{U} \oplus B: U \oplus W_{0} \longrightarrow U \oplus W_{1}
$$


Finally, we check that $L^{*} \Omega_{1}=\Omega_{0}$.

$$
\begin{aligned}
\left(L^{*} \Omega_{1}\right)\left(u \oplus w_{0}, u^{\prime} \oplus w_{0}^{\prime}\right) & =\Omega_{1}\left(u \oplus B w_{0}, u^{\prime} \oplus B \omega_{0}^{\prime}\right) \\
& =\Omega_{1}\left(u, B w_{0}^{\prime}\right)+\Omega_{1}\left(B w_{0}, u^{\prime}\right) \\
& =\Omega_{0}\left(u, w_{0}^{\prime}\right)+\Omega_{0}\left(w_{0}, u^{\prime}\right) \\
& =\Omega_{0}\left(u \oplus w_{0}, u^{\prime} \oplus w_{0}^{\prime}\right) .
\end{aligned}
$$

\subsection{Weinstein Lagrangian Neighborhood Theorem}

Theorem 8.4 (Weinstein Lagrangian Neighborhood Theorem [104]) Let $M$ be a $2 n$-dimensional manifold, $X$ a compact $n$-dimensional submanifold, $i: X \hookrightarrow$ $M$ the inclusion map, and $\omega_{0}$ and $\omega_{1}$ symplectic forms on $M$ such that $i^{*} \omega_{0}=$ $i^{*} \omega_{1}=0$, i.e., $X$ is a lagrangian submanifold of both $\left(M, \omega_{0}\right)$ and $\left(M, \omega_{1}\right)$. Then there exist neighborhoods $\mathcal{U}_{0}$ and $\mathcal{U}_{1}$ of $X$ in $M$ and a diffeomorphism $\varphi: \mathcal{U}_{0} \rightarrow \mathcal{U}_{1}$ such that

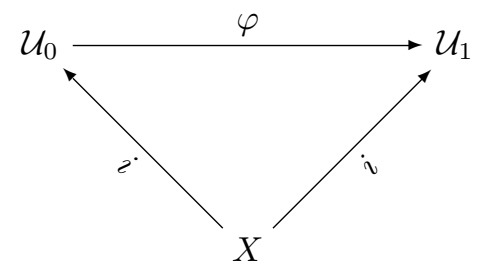

commutes and $\varphi^{*} \omega_{1}=\omega_{0}$.

The proof of the Weinstein theorem uses the Whitney extension theorem.

Theorem 8.5 (Whitney Extension Theorem) Let $M$ be an $n$-dimensional manifold and $X$ a $k$-dimensional submanifold with $k<n$. Suppose that at each $p \in X$ we are given a linear isomorphism $L_{p}: T_{p} M \stackrel{\simeq}{\rightarrow} T_{p} M$ such that $\left.L_{p}\right|_{T_{p} X}=\operatorname{Id}_{T_{p} X}$ and $L_{p}$ depends smoothly on $p$. Then there exists an embedding $h: \mathcal{N} \rightarrow M$ of some neighborhood $\mathcal{N}$ of $X$ in $M$ such that $\left.h\right|_{X}=\operatorname{id}_{X}$ and $d h_{p}=L_{p}$ for all $p \in X$.

The linear maps $L$ serve as "germs" for the embedding.

Proof of the Weinstein theorem. Put a riemannian metric $g$ on $M$; at each $p \in M, g_{p}(\cdot, \cdot)$ is a positive-definite inner product. Fix $p \in X$, and let $V=T_{p} M$, $U=T_{p} X$ and $W=U^{\perp}$ the orthocomplement of $U$ in $V$ relative to $g_{p}(\cdot, \cdot)$.

Since $i^{*} \omega_{0}=i^{*} \omega_{1}=0$, the space $U$ is a lagrangian subspace of both $\left(V,\left.\omega_{0}\right|_{p}\right)$ and $\left(V,\left.\omega_{1}\right|_{p}\right)$. By symplectic linear algebra, we canonically get from $U^{\perp}$ a linear isomorphism $L_{p}: T_{p} M \rightarrow T_{p} M$, such that $\left.L_{p}\right|_{T_{p} X}=\operatorname{Id}_{T_{p} X}$ and $\left.L_{p}^{*} \omega_{1}\right|_{p}=\left.\omega_{0}\right|_{p}$. $L_{p}$ varies smoothly with respect to $p$ since our recipe is canonical!

By the Whitney theorem, there are a neighborhood $\mathcal{N}$ of $X$ and an embedding $h: \mathcal{N} \hookrightarrow M$ with $\left.h\right|_{X}=\operatorname{id}_{X}$ and $d h_{p}=L_{p}$ for $p \in X$. Hence, at any $p \in X$,

$$
\left(h^{*} \omega_{1}\right)_{p}=\left.\left(d h_{p}\right)^{*} \omega_{1}\right|_{p}=\left.L_{p}^{*} \omega_{1}\right|_{p}=\left.\omega_{0}\right|_{p} .
$$


Applying the Moser relative theorem (Theorem 7.4) to $\omega_{0}$ and $h^{*} \omega_{1}$, we find a neighborhood $\mathcal{U}_{0}$ of $X$ and an embedding $f: \mathcal{U}_{0} \rightarrow \mathcal{N}$ such that $\left.f\right|_{X}=\operatorname{id}_{X}$ and $f^{*}\left(h^{*} \omega_{1}\right)=\omega_{0}$ on $\mathcal{U}_{o}$. Set $\varphi=h \circ f$.

\section{Sketch of proof for the Whitney theorem.}

Case $M=\mathbb{R}^{n}$ :

For a compact $k$-dimensional submanifold $X$, take a neighborhood of the form

$$
\mathcal{U}^{\varepsilon}=\{p \in M \mid \text { distance }(p, X) \leq \varepsilon\}
$$

For $\varepsilon$ sufficiently small so that any $p \in \mathcal{U}^{\varepsilon}$ has a unique nearest point in $X$, define a projection $\pi: \mathcal{U}^{\varepsilon} \rightarrow X, p \mapsto$ point on $X$ closest to $p$. If $\pi(p)=q$, then $p=q+v$ for some $v \in N_{q} X$ where $N_{q} X=\left(T_{q} X\right)^{\perp}$ is the normal space at $q$; see Homework 5. Let

$$
\begin{aligned}
h: \mathcal{U}^{\varepsilon} & \longrightarrow \mathbb{R}^{n} \\
p & \longmapsto q+L_{q} v,
\end{aligned}
$$

where $q=\pi(p)$ and $v=p-\pi(p) \in N_{q} X$. Then $h_{X}=\operatorname{id}_{X}$ and $d h_{p}=L_{p}$ for $p \in X$. If $X$ is not compact, replace $\varepsilon$ by a continuous function $\varepsilon: X \rightarrow \mathbb{R}^{+}$which tends to zero fast enough as $x$ tends to infinity.

General case:

Choose a riemannian metric on $M$. Replace distance by riemannian distance, replace straight lines $q+t v$ by geodesics $\exp (q, v)(t)$ and replace $q+L_{q} v$ by the value at $t=1$ of the geodesic with initial value $q$ and initial velocity $L_{q} v$.

In Lecture 30 we will need the following generalization of Theorem 8.4. For a proof see, for instance, either of $[47,58,107]$.

Theorem 8.6 (Coisotropic Embedding Theorem) Let $M$ be a manifold of dimension $2 n, X$ a submanifold of dimension $k \geq n, i: X \hookrightarrow M$ the inclusion map, and $\omega_{0}$ and $\omega_{1}$ symplectic forms on $M$, such that $i^{*} \omega_{0}=i^{*} \omega_{1}$ and $X$ is coisotropic for both $\left(M, \omega_{0}\right)$ and $\left(M, \omega_{1}\right)$. Then there exist neighborhoods $\mathcal{U}_{0}$ and $\mathcal{U}_{1}$ of $X$ in $M$ and a diffeomorphism $\varphi: \mathcal{U}_{0} \rightarrow \mathcal{U}_{1}$ such that

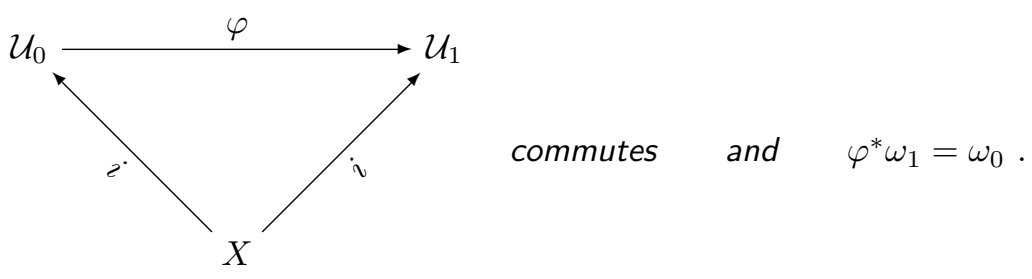




\section{Homework 6: Oriented Surfaces}

1. The standard symplectic form on the 2-sphere is the standard area form:

If we think of $S^{2}$ as the unit sphere in 3-space

$$
S^{2}=\left\{u \in \mathbb{R}^{3} \text { such that }|u|=1\right\},
$$

then the induced area form is given by

$$
\omega_{u}(v, w)=\langle u, v \times w\rangle
$$

where $u \in S^{2}, v, w \in T_{u} S^{2}$ are vectors in $\mathbb{R}^{3}, \times$ is the exterior product, and $\langle\cdot, \cdot\rangle$ is the standard inner product. With this form, the total area of $S^{2}$ is $4 \pi$. Consider cylindrical polar coordinates $(\theta, z)$ on $S^{2}$ away from its poles, where $0 \leq \theta<2 \pi$ and $-1 \leq z \leq 1$.

Show that, in these coordinates,

$$
\omega=d \theta \wedge d z .
$$

2. Prove the Darboux theorem in the 2-dimensional case, using the fact that every nonvanishing 1-form on a surface can be written locally as $f d g$ for suitable functions $f, g$.

Hint: $\omega=d f \wedge d g$ is nondegenerate $\Longleftrightarrow(f, g)$ is a local diffeomorphism.

3. Any oriented 2-dimensional manifold with an area form is a symplectic manifold.

(a) Show that convex combinations of two area forms $\omega_{0}, \omega_{1}$ that induce the same orientation are symplectic.

This is wrong in dimension 4: find two symplectic forms on the vector space $\mathbb{R}^{4}$ that induce the same orientation, yet some convex combination of which is degenerate. Find a path of symplectic forms that connect them.

(b) Suppose that we have two area forms $\omega_{0}, \omega_{1}$ on a compact 2-dimensional manifold $M$ representing the same de Rham cohomology class, i.e., $\left[\omega_{0}\right]=\left[\omega_{1}\right] \in H_{\text {deRham }}^{2}(M)$.

Prove that there is a 1-parameter family of diffeomorphisms $\varphi_{t}: M \rightarrow$ $M$ such that $\varphi_{1}^{*} \omega_{0}=\omega_{1}, \varphi_{0}=\mathrm{id}$, and $\varphi_{t}^{*} \omega_{0}$ is symplectic for all $t \in[0,1]$.

Hint: Exercise (a) and the Moser trick.

Such a 1-parameter family $\varphi_{t}$ is called a strong isotopy between $\omega_{0}$ and $\omega_{1}$. In this language, this exercise shows that, up to strong isotopy, there is a unique symplectic representative in each non-zero 2-cohomology class of $M$. 


\section{Weinstein Tubular Neighborhood Theorem}

\subsection{Observation from Linear Algebra}

Let $(V, \Omega)$ be a symplectic linear space, and let $U$ be a lagrangian subspace.

Claim. There is a canonical nondegenerate bilinear pairing $\Omega^{\prime}: V / U \times U \rightarrow \mathbb{R}$.

Proof. Define $\Omega^{\prime}([v], u)=\Omega(v, u)$ where $[v]$ is the equivalence class of $v$ in $V / U$.

Exercise. Check that $\Omega^{\prime}$ is well-defined and nondegenerate.

Consequently, we get

$\Longrightarrow \widetilde{\Omega^{\prime}}: V / U \rightarrow U^{*}$ defined by $\widetilde{\Omega}^{\prime}([v])=\Omega^{\prime}([v], \cdot)$ is an isomorphism.

$\Longrightarrow V / U \simeq U^{*}$ are canonically identified.

In particular, if $(M, \omega)$ is a symplectic manifold, and $X$ is a lagrangian submanifold, then $T_{x} X$ is a lagrangian subspace of $\left(T_{x} M, \omega_{x}\right)$ for each $x \in X$.

The space $N_{x} X:=T_{x} M / T_{x} X$ is called the normal space of $X$ at $x$.

$\Longrightarrow$ There is a canonical identification $N_{x} X \simeq T_{x}^{*} X$.

$\Longrightarrow$

Theorem 9.1 The vector bundles $N X$ and $T^{*} X$ are canonically identified.

\subsection{Tubular Neighborhoods}

Theorem 9.2 (Standard Tubular Neighborhood Theorem) Let $M$ be an $n$ dimensional manifold, $X$ a $k$-dimensional submanifold, $N X$ the normal bundle of $X$ in $M, i_{0}: X \hookrightarrow N X$ the zero section, and $i: X \hookrightarrow M$ inclusion. Then there are neighborhoods $\mathcal{U}_{0}$ of $X$ in $N X, \mathcal{U}$ of $X$ in $M$ and a diffeomorphism $\psi: \mathcal{U}_{0} \rightarrow \mathcal{U}$ such that

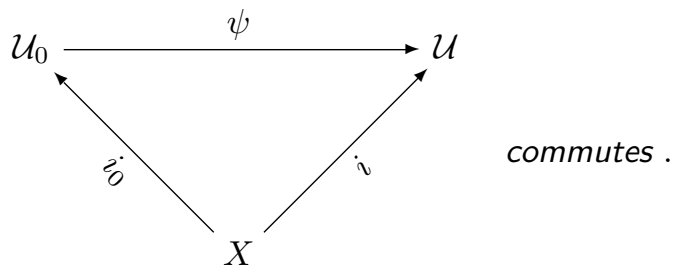

For the proof, see Lecture 6.

Theorem 9.3 (Weinstein Tubular Neighborhood Theorem) Let $(M, \omega)$ be a symplectic manifold, $X$ a compact lagrangian submanifold, $\omega_{0}$ the canonical symplectic form on $T^{*} X, i_{0}: X \hookrightarrow T^{*} X$ the lagrangian embedding as the zero section, and $i: X \hookrightarrow M$ the lagrangian embedding given by inclusion. 
Then there are neighborhoods $\mathcal{U}_{0}$ of $X$ in $T^{*} X, \mathcal{U}$ of $X$ in $M$, and a diffeomorphism $\varphi: \mathcal{U}_{0} \rightarrow \mathcal{U}$ such that

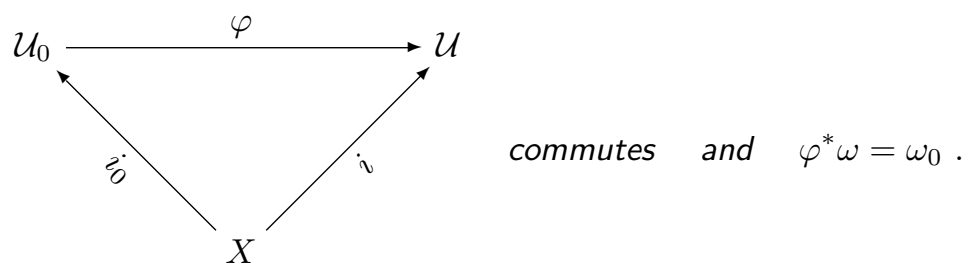

Proof. This proof relies on (1) the standard tubular neighborhood theorem, and (2) the Weinstein lagrangian neighborhood theorem.

(1) Since $N X \simeq T^{*} X$, we can find a neighborhood $\mathcal{N}_{0}$ of $X$ in $T^{*} X$, a neighborhood $\mathcal{N}$ of $X$ in $M$, and a diffeomorphism $\psi: \mathcal{N}_{0} \rightarrow \mathcal{N}$ such that

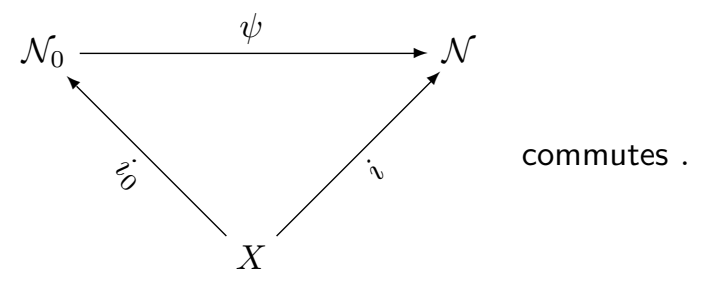

Let $\left.\begin{array}{l}\omega_{0}=\text { canonical form on } T^{*} X \\ \omega_{1}=\psi^{*} \omega\end{array}\right\} \quad$ symplectic forms on $\mathcal{N}_{0}$.

The submanifold $X$ is lagrangian for both $\omega_{0}$ and $\omega_{1}$.

(2) There exist neighborhoods $\mathcal{U}_{0}$ and $\mathcal{U}_{1}$ of $X$ in $\mathcal{N}_{0}$ and a diffeomorphism $\theta: \mathcal{U}_{0} \rightarrow \mathcal{U}_{1}$ such that

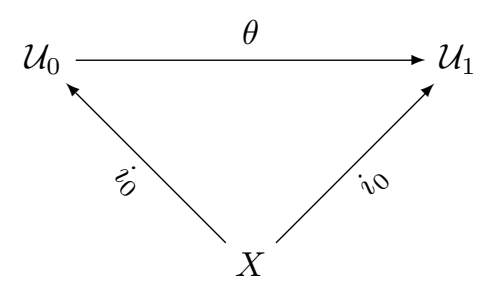

commutes and $\theta^{*} \omega_{1}=\omega_{0}$.

Take $\varphi=\psi \circ \theta$ and $\mathcal{U}=\varphi\left(\mathcal{U}_{0}\right)$. Check that $\varphi^{*} \omega=\theta^{*} \underbrace{\psi^{*} \omega}_{\omega_{1}}=\omega_{0}$.

Remark. Theorem 9.3 classifies lagrangian embeddings: up to local symplectomorphism, the set of lagrangian embeddings is the set of embeddings of manifolds into their cotangent bundles as zero sections. 
The classification of isotropic embeddings was also carried out by Weinstein in $[105,107]$. An isotropic embedding of a manifold $X$ into a symplectic manifold $(M, \omega)$ is a closed embedding $i: X \hookrightarrow M$ such that $i^{*} \omega=0$. Weinstein showed that neighbourhood equivalence of isotropic embeddings is in one-to-one correspondence with isomorphism classes of symplectic vector bundles.

The classification of coisotropic embeddings is due to Gotay [47]. A coisotropic embedding of a manifold $X$ carrying a closed 2-form $\alpha$ of constant rank into a symplectic manifold $(M, \omega)$ is an embedding $i: X \hookrightarrow M$ such that $i^{*} \omega=\alpha$ and $i(X)$ is coisotropic as a submanifold of $M$. Let $E$ be the characteristic distribution of a closed form $\alpha$ of constant rank on $X$, i.e., $E_{p}$ is the kernel of $\alpha_{p}$ at $p \in X$. Gotay showed that then $E^{*}$ carries a symplectic structure in a neighbourhood of the zero section, such that $X$ embeds coisotropically onto this zero section, and, moreover every coisotropic embedding is equivalent to this in some neighbourhood of the zero section.

\subsection{Application 1:}

\section{Tangent Space to the Group of Symplectomorphisms}

The symplectomorphisms of a symplectic manifold $(M, \omega)$ form the group

$$
\operatorname{Sympl}(M, \omega)=\left\{f: M \stackrel{\simeq}{\longrightarrow} M \mid f^{*} \omega=\omega\right\} .
$$

- What is $T_{\text {id }}(\operatorname{Sympl}(M, \omega))$ ?

(What is the "Lie algebra" of the group of symplectomorphisms?)

- What does a neighborhood of id in $\operatorname{Sympl}(M, \omega)$ look like?

We use notions from the $C^{1}$-topology:

$C^{1}$-topology.

Let $X$ and $Y$ be manifolds.

Definition 9.4 A sequence of maps $f_{i}: X \rightarrow Y$ converges in the $C^{\mathbf{0}}$-topology to $f: X \rightarrow Y$ if and only if $f_{i}$ converges uniformly on compact sets.

Definition 9.5 $A$ sequence of $C^{1}$ maps $f_{i}: X \rightarrow Y$ converges in the $C^{\mathbf{1}}$ topology to $f: X \rightarrow Y$ if and only if it and the sequence of derivatives $d f_{i}$ : $T X \rightarrow T Y$ converge uniformly on compact sets.

Let $(M, \omega)$ be a compact symplectic manifold and $f \in \operatorname{Sympl}(M, \omega)$. Then $\left.\begin{array}{l}\text { Graph } f \\ \text { Graph id }=\Delta\end{array}\right\}$ are lagrangian submanifolds of $\left(M \times M, \operatorname{pr}_{1}^{*} \omega-\operatorname{pr}_{2}^{*} \omega\right)$.

( $\operatorname{pr}_{i}: M \times M \rightarrow M, i=1,2$, are the projections to each factor.)

By the Weinstein tubular neighborhood theorem, there exists a neighborhood $\mathcal{U}$ of $\Delta(\simeq M)$ in $\left(M \times M, \operatorname{pr}_{1}^{*} \omega-\operatorname{pr}_{2}^{*} \omega\right)$ which is symplectomorphic to a neighborhood $\mathcal{U}_{0}$ of $M$ in $\left(T^{*} M, \omega_{0}\right)$. Let $\varphi: \mathcal{U} \rightarrow \mathcal{U}_{0}$ be the symplectomorphism satisfying $\varphi(p, p)=(p, 0), \forall p \in M$. 
Suppose that $f$ is sufficiently $\boldsymbol{C}^{\mathbf{1}}$-close to id, i.e., $f$ is in some sufficiently small neighborhood of id in the $C^{1}$-topology. Then:

1. We can assume that Graph $f \subseteq \mathcal{U}$.

Let $\quad j: M \hookrightarrow \mathcal{U} \quad$ be the embedding as Graph $f$, $i: M \hookrightarrow \mathcal{U}$ be the embedding as Graph id $=\Delta$.

2. The map $j$ is sufficiently $C^{1}$-close to $i$.

3. By the Weinstein theorem, $\mathcal{U} \simeq \mathcal{U}_{0} \subseteq T^{*} M$, so the above $j$ and $i$ induce

$$
\begin{array}{ll}
j_{0}: M \hookrightarrow \mathcal{U}_{0} & \text { embedding, where } j_{0}=\varphi \circ j, \\
i_{0}: M \hookrightarrow \mathcal{U}_{0} & \text { embedding as 0-section . }
\end{array}
$$

Hence, we have

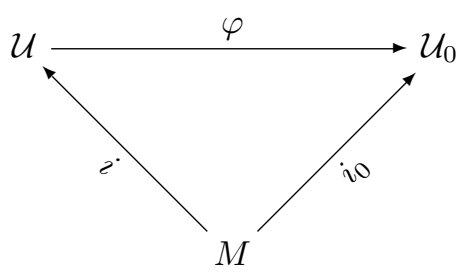

and

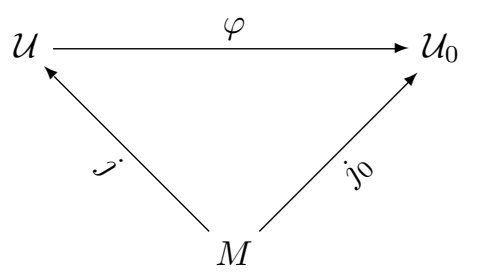

where $i(p)=(p, p), i_{0}(p)=(p, 0), j(p)=(p, f(p))$ and $j_{0}(p)=\varphi(p, f(p))$ for $p \in M$.

4. The map $j_{0}$ is sufficiently $C^{1}$-close to $i_{0}$.

$\Downarrow$

The image set $j_{0}(M)$ intersects each $T_{p}^{*} M$ at one point $\mu_{p}$ depending smoothly on $p$.

5. The image of $j_{0}$ is the image of a smooth section $\mu: M \rightarrow T^{*} M$, that is, a 1 -form $\mu=j_{0} \circ\left(\pi \circ j_{0}\right)^{-1}$.

Therefore, Graph $f \simeq\left\{\left(p, \mu_{p}\right) \mid p \in M, \mu_{p} \in T_{p}^{*} M\right\}$.

Exercise. Vice-versa: if $\mu$ is a 1 -form sufficiently $C^{1}$-close to the zero 1 -form, then

$$
\left\{\left(p, \mu_{p}\right) \mid p \in M, \mu_{p} \in T_{p}^{*} M\right\} \simeq \operatorname{Graph} f,
$$

for some diffeomorphism $f: M \rightarrow M$. By Lecture 3, we have

Graph $f$ is lagrangian $\Longleftrightarrow \mu$ is closed.

Conclusion. A small $C^{1}$-neighborhood of id in $\operatorname{Sympl}(M, \omega)$ is homeomorphic to a $C^{1}$-neighborhood of zero in the vector space of closed 1 -forms on $M$. So:

$$
T_{\mathrm{id}}(\operatorname{Sympl}(M, \omega)) \simeq\left\{\mu \in \Omega^{1}(M) \mid d \mu=0\right\} .
$$

In particular, $T_{\mathrm{id}}(\operatorname{Sympl}(M, \omega))$ contains the space of exact 1-forms

$$
\left\{\mu=d h \mid h \in C^{\infty}(M)\right\} \simeq C^{\infty}(M) / \text { locally constant functions } .
$$




\subsection{Application 2:}

\section{Fixed Points of Symplectomorphisms}

Theorem 9.6 Let $(M, \omega)$ be a compact symplectic manifold with $H_{\mathrm{deRham}}^{1}(M)=$ 0 . Then any symplectomorphism of $M$ which is sufficiently $C^{1}$-close to the identity has at least two fixed points.

Proof. Suppose that $f \in \operatorname{Sympl}(M, \omega)$ is sufficiently $C^{1}$-close to id.

Then Graph $f \simeq$ closed 1-form $\mu$ on $M$.

$$
\left.\begin{array}{l}
d \mu=0 \\
H_{\text {deRham }}^{1}(M)=0
\end{array}\right\} \Longrightarrow \mu=d h \text { for some } h \in C^{\infty}(M) .
$$

Since $M$ is compact, $h$ has at least 2 critical points.

$$
\begin{array}{ccc}
\begin{array}{c}
\text { Fixed points of } \mathrm{f} \\
\|
\end{array} & \quad \text { critical points of } h \\
\text { Graph } f \cap \Delta & =\left\{p: \mu_{p}=d h_{p}=0\right\} .
\end{array}
$$

\section{Lagrangian intersection problem:}

A submanifold $Y$ of $M$ is $\boldsymbol{C}^{\mathbf{1}}$-close to $X$ when there is a diffeomorphism $X \rightarrow Y$ which is, as a map into $M, C^{1}$-close to the inclusion $X \hookrightarrow M$.

Theorem 9.7 Let $(M, \omega)$ be a symplectic manifold. Suppose that $X$ is a compact lagrangian submanifold of $M$ with $H_{\mathrm{deRham}}^{1}(X)=0$. Then every lagrangian submanifold of $M$ which is $C^{1}$-close to $X$ intersects $X$ in at least two points.

Proof. Exercise.

\section{Arnold conjecture:}

Let $(M, \omega)$ be a compact symplectic manifold, and $f: M \rightarrow M$ a symplectomorphism which is "exactly homotopic to the identity" (see below). Then

$$
\begin{aligned}
\#\{\text { fixed points of } f\} \geq & \begin{array}{l}
\text { minimal \# of critical points } \\
\text { a smooth function on } M \text { can have. }
\end{array}
\end{aligned}
$$

Together with Morse theory, ${ }^{8}$ we obtain ${ }^{9}$

$$
\begin{aligned}
\#\{\text { nondegenerate fixed points of } f\} \geq & \text { minimal \# of critical points } \\
& \text { a Morse function on } M \text { can have } \\
\geq & \sum_{i=0}^{2 n} \operatorname{dim} H^{i}(M ; \mathbb{R}) .
\end{aligned}
$$

\footnotetext{
${ }^{8}$ A Morse function on $M$ is a function $h: M \rightarrow \mathbb{R}$ whose critical points (i.e., points $p$ where $\left.d h_{p}=0\right)$ are all nondegenerate (i.e., the hessian at those points is nonsingular: $\left.\operatorname{det}\left(\frac{\partial^{2} h}{\partial x_{i} \partial x_{j}}\right)_{p} \neq 0\right)$.

${ }^{9} \mathrm{~A}$ fixed point $p$ of $f: M \rightarrow M$ is nondegenerate if $d f_{p}: T_{p} M \rightarrow T_{p} M$ is nonsingular.
} 
The Arnold conjecture was proved by Conley-Zehnder, Floer, Hofer-Salamon, Ono, Fukaya-Ono, Liu-Tian using Floer homology (which is an $\infty$-dimensional analogue of Morse theory). There are open conjectures for sharper bounds on the number of fixed points.

Meaning of " $f$ is exactly homotopic to the identity:"

Suppose that $h_{t}: M \rightarrow \mathbb{R}$ is a smooth family of functions which is 1-periodic, i.e., $h_{t}=h_{t+1}$. Let $\rho: M \times \mathbb{R} \rightarrow M$ be the isotopy generated by the time-dependent vector field $v_{t}$ defined by $\omega\left(v_{t}, \cdot\right)=d h_{t}$. Then " $f$ being exactly homotopic to the identity" means $f=\rho_{1}$ for some such $h_{t}$.

In other words, $f$ is exactly homotopic to the identity when $f$ is the time- 1 map of an isotopy generated by some smooth time-dependent 1 -periodic hamiltonian function.

There is a one-to-one correspondence

$$
\text { fixed points of } f \stackrel{1-1}{\longleftrightarrow} \text { period-1 orbits of } \rho: M \times \mathbb{R} \rightarrow M
$$

because $f(p)=p$ if and only if $\{\rho(t, p), t \in[0,1]\}$ is a closed orbit.

Proof of the Arnold conjecture in the case when $h: M \rightarrow \mathbb{R}$ is independent of $t$ : $p$ is a critical point of $h \Longleftrightarrow d h_{p}=0 \Longleftrightarrow v_{p}=0$

$\Longrightarrow \rho(t, p)=p, \forall t \in \mathbb{R} \Longrightarrow p$ is a fixed point of $\rho_{1}$.

Exercise. Compute these estimates for the number of fixed points on some compact symplectic manifolds (for instance, $S^{2}, S^{2} \times S^{2}$ and $T^{2}=S^{1} \times S^{1}$ ). 


\section{Part IV}

\section{Contact Manifolds}

Contact geometry is also known as "the odd-dimensional analogue of symplectic geometry." We will browse through the basics of contact manifolds and their relation to symplectic manifolds.

\section{Contact Forms}

\subsection{Contact Structures}

Definition 10.1 A contact element on a manifold $M$ is a point $p \in M$, called the contact point, together with a tangent hyperplane at $p, H_{p} \subset T_{p} M$, that is, a codimension-1 subspace of $T_{p} M$.

A hyperplane $H_{p} \subset T_{p} M$ determines a covector $\alpha_{p} \in T_{p}^{*} M \backslash\{0\}$, up to multiplication by a nonzero scalar:

$\left(p, H_{p}\right)$ is a contact element $\longleftrightarrow H_{p}=\operatorname{ker} \alpha_{p}$ with $\alpha_{p}: T_{p} M \longrightarrow \mathbb{R}$ linear,$\neq 0$

$$
\text { ker } \alpha_{p}=\operatorname{ker} \alpha_{p}^{\prime} \Longleftrightarrow \alpha_{p}=\lambda \alpha_{p}^{\prime} \text { for some } \lambda \in \mathbb{R} \backslash\{0\} .
$$

Suppose that $H$ is a smooth field of contact elements (i.e., of tangent hyperplanes) on $M$ :

$$
H: p \longmapsto H_{p} \subset T_{p} M
$$

Locally, $H=\operatorname{ker} \alpha$ for some 1-form $\alpha$, called a locally defining 1-form for $H$. ( $\alpha$ is not unique: $\operatorname{ker} \alpha=\operatorname{ker}(f \alpha)$, for any nowhere vanishing $f: M \rightarrow \mathbb{R}$.)

Definition 10.2 A contact structure on $M$ is a smooth field of tangent hyperplanes $H \subset T M$, such that, for any locally defining 1-form $\alpha$, we have $\left.d \alpha\right|_{H}$ nondegenerate (i.e., symplectic). The pair $(M, H)$ is then called a contact manifold and $\alpha$ is called a local contact form.

At each $p \in M$,

$$
T_{p} M=\underbrace{\operatorname{ker} \alpha_{p}}_{H_{p}} \oplus \underbrace{\operatorname{ker} d \alpha_{p}}_{1-\text { dimensional }} .
$$

The ker $d \alpha_{p}$ summand in this splitting depends on the choice of $\alpha$.

$$
\begin{aligned}
\left.d \alpha_{p}\right|_{H_{p}} \text { nondegenerate } \\
\left.\alpha_{p}\right|_{\text {ker } d \alpha_{p}} \text { nondegenerate }
\end{aligned} \Longrightarrow \begin{cases}\operatorname{dim} H_{p}=2 n & \text { is even } \\
\left.\left(d \alpha_{p}\right)^{n}\right|_{H_{p}} \neq 0 & \text { is a volume form on } H_{p}\end{cases}
$$

Therefore,

- any contact manifold $(M, H)$ has $\operatorname{dim} M=2 n+1$ odd, and 
- if $\alpha$ is a (global) contact form, then $\alpha \wedge(d \alpha)^{n}$ is a volume form on $M$.

Remark. Let $(M, H)$ be a contact manifold. A global contact form exists if and only if the quotient line bundle $T M / H$ is orientable. Since $H$ is also orientable, this implies that $M$ is orientable.

Proposition 10.3 Let $H$ be a field of tangent hyperplanes on $M$. Then

$H$ is a contact structure $\Longleftrightarrow \alpha \wedge(d \alpha)^{n} \neq 0$ for every locally defining 1-form $\alpha$.

\section{Proof.}

$\Longrightarrow$ Done above.

$\Longleftarrow$ Suppose that $H=\operatorname{ker} \alpha$ locally. We need to show:

$$
\left.d \alpha\right|_{H} \text { nondegenerate } \Longleftrightarrow \alpha \wedge(d \alpha)^{n} \neq 0 .
$$

Take a local trivialization $\left\{e_{1}, f_{1}, \ldots, e_{n}, f_{n}, r\right\}$ of $T M=\operatorname{ker} \alpha \oplus$ rest , such that $\operatorname{ker} \alpha=\operatorname{span}\left\{e_{1}, f_{1}, \ldots, e_{n}, f_{n}\right\}$ and rest $=\operatorname{span}\{r\}$.

$$
\left(\alpha \wedge(d \alpha)^{n}\right)\left(e_{1}, f_{1}, \ldots, e_{n}, f_{n}, r\right)=\underbrace{\alpha(r)}_{\neq 0} \cdot(d \alpha)^{n}\left(e_{1}, f_{1}, \ldots, e_{n}, f_{n}\right)
$$

and hence $\alpha \wedge(d \alpha)^{n} \neq\left. 0 \Longleftrightarrow(d \alpha)^{n}\right|_{H} \neq\left. 0 \Longleftrightarrow d \alpha\right|_{H}$ is nondegenerate .

\subsection{Examples}

1. On $\mathbb{R}^{3}$ with coordinates $(x, y, z)$, consider $\alpha=x d y+d z$. Since

$$
\alpha \wedge d \alpha=(x d y+d z) \wedge(d x \wedge d y)=d x \wedge d y \wedge d z \neq 0,
$$

$\alpha$ is a contact form on $\mathbb{R}^{3}$.

The corresponding field of hyperplanes $H=\operatorname{ker} \alpha$ at $(x, y, z) \in \mathbb{R}^{3}$ is

$$
H_{(x, y, z)}=\left\{v=a \frac{\partial}{\partial x}+b \frac{\partial}{\partial y}+c \frac{\partial}{\partial z} \mid \alpha(v)=b x+c=0\right\} .
$$

Exercise. Picture these hyperplanes.

2. (Martinet [80], 1971) Any compact orientable 3-manifold admits a contact structure.

Open Problem, 2000. The classification of compact orientable contact 3manifolds is still not known. There is by now a huge collection of results in contact topology related to the classification of contact manifolds. For a review of the state of the knowledge and interesting questions on contact 3-manifolds, see [33, 43, 100]. 
3. Let $X$ be a manifold and $T^{*} X$ its cotangent bundle. There are two canonical contact manifolds associated to $X$ (see Homework 7 ):

$$
\begin{aligned}
& \mathbb{P}\left(T^{*} X\right)=\text { the projectivization of } T^{*} X \text {, and } \\
& S\left(T^{*} X\right)=\text { the cotangent sphere bundle. }
\end{aligned}
$$

4. On $\mathbb{R}^{2 n+1}$ with coordinates $\left(x_{1}, y_{1}, \ldots, x_{n}, y_{n}, z\right), \alpha=\sum_{i} x_{i} d y_{i}+d z$ is contact.

\subsection{First Properties}

There is a local normal form theorem for contact manifolds analogous to the Darboux theorem for symplectic manifolds.

Theorem 10.4 Let $(M, H)$ be a contact manifold and $p \in M$. Then there exists a coordinate system $\left(\mathcal{U}, x_{1}, y_{1}, \ldots, x_{n}, y_{n}, z\right)$ centered at $p$ such that on $\mathcal{U}$

$$
\alpha=\sum x_{i} d y_{i}+d z \text { is a local contact form for } H \text {. }
$$

The idea behind the proof is sketched in the next lecture.

There is also a Moser-type theorem for contact forms.

Theorem 10.5 (Gray) Let $M$ be a compact manifold. Suppose that $\alpha_{t}, t \in[0,1]$, is a smooth family of (global) contact forms on $M$. Let $H_{t}=\operatorname{ker} \alpha_{t}$. Then there exists an isotopy $\rho: M \times \mathbb{R} \longrightarrow M$ such that $H_{t}=\rho_{t *} H_{0}$, for all $0 \leq t \leq 1$.

Exercise. Show that $H_{t}=\rho_{t *} H_{0} \Longleftrightarrow \rho_{t}^{*} \alpha_{t}=u_{t} \cdot \alpha_{0}$ for some family $u_{t}: M \longrightarrow$ $\mathbb{R}, 0 \leq t \leq 1$, of nowhere vanishing functions.

Proof. (À la Moser)

We need to find $\rho_{t}$ such that $\left\{\begin{array}{l}\rho_{0}=\mathrm{id} \\ \frac{d}{d t}\left(\rho_{t}^{*} \alpha_{t}\right)=\frac{d}{d t}\left(u_{t} \alpha_{0}\right) . \quad \text { For any isotopy } \rho,\end{array}\right.$

$$
\frac{d}{d t}\left(\rho_{t}^{*} \alpha_{t}\right)=\rho_{t}^{*}\left(\mathcal{L}_{v_{t}} \alpha_{t}+\frac{d \alpha_{t}}{d t}\right)
$$

where $v_{t}=\frac{d \rho_{t}}{d t} \circ \rho_{t}^{-1}$ is the vector field generated by $\rho_{t}$. By the Moser trick, it suffices to find $v_{t}$ and then integrate it to $\rho_{t}$. We will search for $v_{t}$ in $H_{t}=\operatorname{ker} \alpha_{t}$; this unnecessary assumption simplifies the proof. 
We need to solve

$$
\begin{array}{rlrl} 
& \rho_{t}^{*}(\underbrace{\mathcal{L}_{v_{t}} \alpha_{t}}_{d v_{v_{t}} \alpha_{t}+v_{v_{t}} d \alpha_{t}}+\frac{d \alpha_{t}}{d t}) & =\frac{d u_{t}}{d t} \underbrace{\alpha_{0}}_{\frac{1}{u_{t}} \rho_{t}^{*} \alpha_{t}} \\
\Longrightarrow \quad \rho_{t}^{*}\left(\imath_{v_{t}} d \alpha_{t}+\frac{d \alpha_{t}}{d t}\right) & =\frac{d u_{t}}{d t} \cdot \frac{1}{u_{t}} \cdot \rho_{t}^{*} \alpha_{t} \\
\Longleftrightarrow \quad v_{v_{t}} d \alpha_{t}+\frac{d \alpha_{t}}{d t} & =\left(\rho_{t}^{*}\right)^{-1}\left(\frac{d u_{t}}{d t} \cdot \frac{1}{u_{t}}\right) \alpha_{t} .
\end{array}
$$

Restricting to the hyperplane $H_{t}=\operatorname{ker} \alpha_{t}$, equation $(\star)$ reads

$$
\left.\imath_{v_{t}} d \alpha_{t}\right|_{H_{t}}=-\left.\frac{d \alpha_{t}}{d t}\right|_{H_{t}}
$$

which determines $v_{t}$ uniquely, since $\left.d \alpha_{t}\right|_{H_{t}}$ is nondegenerate. After integrating $v_{t}$ to $\rho_{t}$, the factor $u_{t}$ is determined by the relation $\rho_{t}^{*} \alpha_{t}=u_{t} \cdot \alpha_{0}$. Check that this indeed gives a solution. 


\section{Homework 7: Manifolds of Contact Elements}

Given any manifold $X$ of dimension $n$, there is a canonical symplectic manifold of dimension $2 n$ attached to it, namely its cotangent bundle with the standard symplectic structure. The exercises below show that there is also a canonical contact manifold of dimension $2 n-1$ attached to $X$.

The manifold of contact elements of an $n$-dimensional manifold $X$ is

$$
\mathcal{C}=\left\{\left(x, \chi_{x}\right) \mid x \in X \text { and } \chi_{x} \text { is a hyperplane in } T_{x} X\right\} .
$$

On the other hand, the projectivization of the cotangent bundle of $X$ is

$$
\mathbb{P}^{*} X=\left(T^{*} X \backslash \text { zero section }\right) / \sim
$$

where $(x, \xi) \sim\left(x, \xi^{\prime}\right)$ whenever $\xi=\lambda \xi^{\prime}$ for some $\lambda \in \mathbb{R} \backslash\{0\}$ (here $x \in X$ and $\left.\xi, \xi^{\prime} \in T_{x}^{*} X \backslash\{0\}\right)$. We will denote elements of $\mathbb{P}^{*} X$ by $(x,[\xi])$, [ $[\xi]$ being the $~$ equivalence class of $\xi$.

1. Show that $\mathcal{C}$ is naturally isomorphic to $\mathbb{P}^{*} X$ as a bundle over $X$, i.e., exhibit a diffeomorphism $\varphi: \mathcal{C} \rightarrow \mathbb{P}^{*} X$ such that the following diagram commutes:

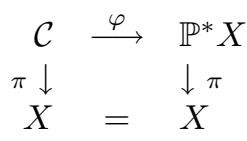

where the vertical maps are the natural projections $\left(x, \chi_{x}\right) \mapsto x$ and $(x, \xi) \mapsto$ $x$.

Hint: The kernel of a non-zero $\xi \in T_{x}^{*} X$ is a hyperplane $\chi_{x} \subset T_{x} X$.

What is the relation between $\xi$ and $\xi^{\prime}$ if $\operatorname{ker} \xi=\operatorname{ker} \xi^{\prime}$ ?

2. There is on $\mathcal{C}$ a canonical field of hyperplanes $\mathcal{H}$ (that is, a smooth map attaching to each point in $\mathcal{C}$ a hyperplane in the tangent space to $\mathcal{C}$ at that point): $\mathcal{H}$ at the point $p=\left(x, \chi_{x}\right) \in \mathcal{C}$ is the hyperplane

$$
\mathcal{H}_{p}=\left(d \pi_{p}\right)^{-1} \chi_{x} \subset T_{p} \mathcal{C}
$$

where

$$
\begin{array}{ccc}
\mathcal{C} & p=\left(x, \chi_{x}\right) & T_{p} \mathcal{C} \\
\downarrow \pi & \downarrow & \downarrow d \pi_{p} \\
X & x & T_{x} X
\end{array}
$$

are the natural projections, and $\left(d \pi_{p}\right)^{-1} \chi_{x}$ is the preimage of $\chi_{x} \subset T_{x} X$ by $d \pi_{p}$.

Under the isomorphism $\mathcal{C} \simeq \mathbb{P}^{*} X$ from exercise $1, \mathcal{H}$ induces a field of hyperplanes $\mathbb{H}$ on $\mathbb{P}^{*} X$. Describe $\mathbb{H}$.

Hint: If $\xi \in T_{x}^{*} X \backslash\{0\}$ has kernel $\chi_{x}$, what is the kernel of the canonical 1-form $\alpha_{(x, \xi)}=\left(d \pi_{(x, \xi)}\right) * \xi ?$ 
3. Check that $\left(\mathbb{P}^{*} X, \mathbb{H}\right)$ is a contact manifold, and therefore $(\mathcal{C}, \mathcal{H})$ is a contact manifold.

Hint: Let $(x,[\xi]) \in \mathbb{P}^{*} X$. For any $\xi$ representing the class $[\xi]$, we have

$$
\mathbb{H}_{(x,[\xi])}=\operatorname{ker}\left(\left(d \pi_{(x,[\xi])}\right)^{*} \xi\right) .
$$

Let $x_{1}, \ldots, x_{n}$ be local coordinates on $X$, and let $x_{1}, \ldots, x_{n}, \xi_{1}, \ldots, \xi_{n}$ be the associated local coordinates on $T^{*} X$. In these coordinates, $(x,[\xi])$ is given by $\left(x_{1}, \ldots, x_{n},\left[\xi_{1}, \ldots, \xi_{n}\right]\right)$. Since at least one of the $\xi_{i}$ 's is nonzero, without loss of generality we may assume that $\xi_{1} \neq 0$ so that we may divide $\xi$ by $\xi_{1}$ to obtain a representative with coordinates $\left(1, \xi_{2}, \ldots, \xi_{n}\right)$. Hence, by choosing always the representative of $[\xi]$ with $\xi_{1}=1$, the set $x_{1}, \ldots, x_{n}, \xi_{2}, \ldots, \xi_{n}$ defines coordinates on some neighborhood $\mathcal{U}$ of $(x,[\xi])$ in $\mathbb{P}^{*} X$. On $\mathcal{U}$, consider the 1 -form

$$
\alpha=d x_{1}+\sum_{i \geq 2} \xi_{i} d x_{i}
$$

Show that $\alpha$ is a contact form on $\mathcal{U}$, i.e., show that $\operatorname{ker} \alpha_{(x,[\xi])}=\mathbb{H}_{(x,[\xi])}$, and that $d \alpha_{(x,[\xi])}$ is nondegenerate on $\mathbb{H}_{(x,[\xi])}$.

4. What is the symplectization of $\mathcal{C}$ ?

What is the manifold $\mathcal{C}$ when $X=\mathbb{R}^{3}$ and when $X=S^{1} \times S^{1}$ ?

Remark. Similarly, we could have defined the manifold of oriented contact elements of $X$ to be

$$
\mathcal{C}^{o}=\left\{\left(x, \chi_{x}^{o}\right) \mid x \in X \text { and } \begin{array}{c}
\chi_{x}^{o} \text { is a hyperplane in } T_{x} X \\
\text { equipped with an orientation }
\end{array}\right\} .
$$

The manifold $\mathcal{C}^{o}$ is isomorphic to the cotangent sphere bundle of $X$

$$
S^{*} X:=\left(T^{*} X \backslash \text { zero section }\right) / \approx
$$

where $(x, \xi) \approx\left(x, \xi^{\prime}\right)$ whenever $\xi=\lambda \xi^{\prime}$ for some $\lambda \in \mathbb{R}^{+}$.

A construction analogous to the above produces a canonical contact structure on $\mathcal{C}^{\circ}$. See [3, Appendix 4]. 


\section{Contact Dynamics}

\subsection{Reeb Vector Fields}

Let $(M, H)$ be a contact manifold with a contact form $\alpha$.

Claim. There exists a unique vector field $R$ on $M$ such that $\left\{\begin{aligned} \imath_{R} d \alpha & =0 \\ \imath_{R} \alpha & =1\end{aligned}\right.$

Proof. $\left\{\begin{array}{lll}\imath_{R} d \alpha=0 & \Longrightarrow & R \in \text { ker } d \alpha, \text { which is a line bundle, and } \\ \imath_{R} \alpha=1 & \Longrightarrow & \text { normalizes } R .\end{array}\right.$

The vector field $R$ is called the Reeb vector field determined by $\alpha$.

Claim. The flow of $R$ preserves the contact form, i.e., if $\rho_{t}=\exp t R$ is the isotopy generated by $R$, then $\rho_{t}^{*} \alpha=\alpha, \forall t \in \mathbb{R}$.

Proof. We have $\frac{d}{d t}\left(\rho_{t}^{*} \alpha\right)=\rho_{t}^{*}\left(\mathcal{L}_{R} \alpha\right)=\rho_{t}^{*}(\underbrace{\imath_{R} \alpha}_{1}+\underbrace{\imath_{R} d \alpha}_{0})=0$.

Hence, $\rho_{t}^{*} \alpha=\rho_{0}^{*} \alpha=\alpha, \forall t \in \mathbb{R}$.

Definition 11.1 A contactomorphism is a diffeomorphism $f$ of a contact manifold $(M, H)$ which preserves the contact structure (i.e., $f_{*} H=H$ ).

\section{Examples.}

1. Euclidean space $\mathbb{R}^{2 n+1}$ with $\alpha=\sum_{i} x_{i} d y_{i}+d z$.

$$
\left.\begin{array}{rl}
\imath_{R} \sum d x_{i} \wedge d y_{i} & =0 \\
\imath_{R} \sum x_{i} d y_{i}+d z & =1
\end{array}\right\} \Longrightarrow R=\frac{\partial}{\partial z} \text { is the Reeb vector field. }
$$

The contactomorphisms generated by $R$ are translations

$$
\rho_{t}\left(x_{1}, y_{1}, \ldots, x_{n}, y_{n}, z\right)=\left(x_{1}, y_{1}, \ldots, x_{n}, y_{n}, z+t\right) \text {. }
$$

2. Regard the odd sphere $S^{2 n-1} \stackrel{i}{\hookrightarrow} \mathbb{R}^{2 n}$ as the set of unit vectors

$$
\left\{\left(x_{1}, y_{1}, \ldots, x_{n}, y_{n}\right) \mid \sum\left(x_{i}^{2}+y_{i}^{2}\right)=1\right\} .
$$

Consider the 1 -form on $\mathbb{R}^{2 n}, \sigma=\frac{1}{2} \sum\left(x_{i} d y_{i}-y_{i} d x_{i}\right)$.

Claim. The form $\alpha=i^{*} \sigma$ is a contact form on $S^{2 n-1}$.

Proof. We need to show that $\alpha \wedge(d \alpha)^{n-1} \neq 0$. The 1 -form on $\mathbb{R}^{2 n} \nu=$ $d \sum\left(x_{i}^{2}+y_{i}^{2}\right)=2 \sum\left(x_{i} d x_{i}+y_{i} d y_{i}\right)$ satisfies $T_{p} S^{2 n-1}=\operatorname{ker} \nu_{p}$, at $p \in S^{2 n-1}$. Check that $\nu \wedge \sigma \wedge(d \sigma)^{n-1} \neq 0$. 
The distribution $H=\operatorname{ker} \alpha$ is called the standard contact structure on $S^{2 n-1}$. The Reeb vector field is $R=2 \sum\left(x_{i} \frac{\partial}{\partial y_{i}}-y_{i} \frac{\partial}{\partial x_{i}}\right)$, and is also known as the Hopf vector field on $S^{2 n-1}$, as the orbits of its flow are the circles of the Hopf fibration.

\subsection{Symplectization}

Example. Let $\widetilde{M}=S^{2 n-1} \times \mathbb{R}$, with coordinate $\tau$ in the $\mathbb{R}$-factor, and projection $\pi: \widetilde{M} \rightarrow S^{2 n-1},(p, \tau) \mapsto p$. Under the identification $\widetilde{M} \simeq \mathbb{R}^{2 n} \backslash\{0\}$, where the $\mathbb{R}$-factor represents the logarithm of the square of the radius, the projection $\pi$ becomes

$$
\pi: \begin{array}{ccc}
\mathbb{R}^{2 n} \backslash\{0\} & \longrightarrow & S^{2 n-1} \\
\left(X_{1}, Y_{1}, \ldots, X_{n}, Y_{n}\right) & \longmapsto & \left(\frac{X_{1}}{\sqrt{e^{\tau}}}, \frac{Y_{1}}{\sqrt{e^{\tau}}}, \ldots, \frac{X_{n}}{\sqrt{e^{\tau}}}, \frac{Y_{n}}{\sqrt{e^{\tau}}}\right)
\end{array}
$$

where $e^{\tau}=\sum\left(X_{i}^{2}+Y_{i}^{2}\right)$. Let $\alpha=i^{*} \sigma$ be the standard contact form on $S^{2 n-1}$ (see the previous example). Then $\omega=d\left(e^{\tau} \pi^{*} \alpha\right)$ is a closed 2-form on $\mathbb{R}^{2 n} \backslash\{0\}$. Since $\pi^{*} i^{*} x_{i}=\frac{X_{i}}{\sqrt{e^{\tau}}}, \pi^{*} i^{*} y_{i}=\frac{Y_{i}}{\sqrt{e^{\tau}}}$, we have

$$
\begin{aligned}
\pi^{*} \alpha=\pi^{*} i^{*} \sigma & =\frac{1}{2} \sum\left(\frac{X_{i}}{\sqrt{e^{\tau}}} d\left(\frac{Y_{i}}{\sqrt{e^{\tau}}}\right)-\frac{Y_{i}}{\sqrt{e^{\tau}}} d\left(\frac{X_{i}}{\sqrt{e^{\tau}}}\right)\right) \\
& =\frac{1}{2 e^{\tau}} \sum\left(X_{i} d Y_{i}-Y_{i} d X_{i}\right) .
\end{aligned}
$$

Therefore, $\omega=\sum d X_{i} \wedge d Y_{i}$ is the standard symplectic form on $\mathbb{R}^{2 n} \backslash\{0\} \subset \mathbb{R}^{2 n}$. $(\widetilde{M}, \omega)$ is called the symplectization of $\left(S^{2 n-1}, \alpha\right)$.

Proposition 11.2 Let $(M, H)$ be a contact manifold with a contact form $\alpha$. Let $\widetilde{M}=M \times \mathbb{R}$, and let $\pi: \widetilde{M} \rightarrow M,(p, \tau) \mapsto p$, be the projection. Then $\omega=$ $d\left(e^{\tau} \pi^{*} \alpha\right)$ is a symplectic form on $\widetilde{M}$, where $\tau$ is a coordinate on $\mathbb{R}$.

Proof. Exercise.

Hence, $\widetilde{M}$ has a symplectic form $\omega$ canonically determined by a contact form $\alpha$ on $M$ and a coordinate function on $\mathbb{R} ;(\widetilde{M}, \omega)$ is called the symplectization of $(M, \alpha)$.

\section{Remarks.}

1. The contact version of the Darboux theorem can now be derived by applying the symplectic theorem to the symplectization of the contact manifold (with appropriate choice of coordinates); see [3, Appendix 4]. 
2. There is a coordinate-free description of $\widetilde{M}$ as

$$
\widetilde{M}=\left\{(p, \xi) \mid p \in M, \xi \in T_{p}^{*} M, \text { such that } \operatorname{ker} \xi=H_{p}\right\} .
$$

The group $\mathbb{R} \backslash\{0\}$ acts on $\widetilde{M}$ by multiplication on the cotangent vector:

$$
\lambda \cdot(p, \xi)=(p, \lambda \xi), \quad \lambda \in \mathbb{R} \backslash\{0\} .
$$

The quotient $\widetilde{M} /(\mathbb{R} \backslash\{0\})$ is diffeomorphic to $M$. $\widetilde{M}$ has a canonical 1-form $\widetilde{\alpha}$ defined at $v \in T_{(p, \xi)} \widetilde{M}$ by

$$
\widetilde{\alpha}_{(p, \xi)}(v)=\xi\left((d \mathrm{pr})_{(p, \xi)} v\right),
$$

where $\operatorname{pr}: \widetilde{M} \rightarrow M$ is the bundle projection.

\subsection{Conjectures of Seifert and Weinstein}

Question. (Seifert, 1948) Let $v$ be a nowhere vanishing vector field on the 3 -sphere. Does the flow of $v$ have any periodic orbits?

\section{Counterexamples.}

- (Schweitzer, 1974) $\exists C^{1}$ vector field without periodic orbits.

- (Kristina Kuperberg, 1994) $\exists C^{\infty}$ vector field without periodic orbits.

Question. How about volume-preserving vector fields?

- (Greg Kuperberg, 1997) $\exists C^{1}$ counterexample.

- $C^{\infty}$ counterexamples are not known.

Natural generalization of this problem:

Let $M=S^{3}$ be the 3-sphere, and let $\gamma$ be a volume form on $M$. Suppose that $v$ is a nowhere vanishing vector field, and suppose that $v$ is volume-preserving, i.e.,

$$
\mathcal{L}_{v} \gamma=0 \Longleftrightarrow d \imath_{v} \gamma=0 \Longleftrightarrow \imath_{v} \gamma=d \alpha
$$

for some 1-form $\alpha$, since $H^{2}\left(S^{3}\right)=0$.

Given a 1 -form $\alpha$, we would like to study vector fields $v$ such that

$$
\left\{\begin{array}{l}
\imath_{v} \gamma=d \alpha \\
\imath_{v} \alpha>0
\end{array}\right.
$$


A vector field $v$ satisfying $\imath_{v} \alpha>0$ is called positive. For instance, vector fields in a neighborhood of the Hopf vector field are positive relative to the standard contact form on $S^{3}$.

Renormalizing as $R:=\frac{v}{\imath_{v} \alpha}$, we should study instead

$$
\left\{\begin{array}{l}
\imath_{R} d \alpha=0 \\
\imath_{\alpha}=1 \\
\alpha \wedge d \alpha \text { is a volume form, }
\end{array}\right.
$$

that is, study pairs $(\alpha, R)$ where

$$
\left\{\begin{array}{l}
\alpha \text { is a contact form, and } \\
R \text { is its Reeb vector field. }
\end{array}\right.
$$

Conjecture. (Weinstein, 1978 [106]) Suppose that $M$ is a 3-dimensional manifold with a (global) contact form $\alpha$. Let $v$ be the Reeb vector field for $\alpha$. Then $v$ has a periodic orbit.

Theorem 11.3 (Viterbo and Hofer, 1993 [63, 64, 103]) The Weinstein conjecture is true when

1) $M=S^{3}$, or

2) $\pi_{2}(M) \neq 0$, or

3) the contact structure is overtwisted. ${ }^{10}$

\section{Open questions.}

- How many periodic orbits are there?

- What do they look like?

- Is there always an unknotted one?

- What about the linking behavior?

\footnotetext{
${ }^{10}$ A surface $S$ inside a contact 3-manifold determines a singular foliation on $S$, called the characteristic foliation of $S$, by the intersection of the contact planes with the tangent spaces to $S$. A contact structure on a 3-manifold $M$ is called overtwisted if there exists an embedded 2-disk whose characteristic foliation contains one closed leaf $C$ and exactly one singular point inside $C$; otherwise, the contact structure is called tight. Eliashberg [32] showed that the isotopy classification of overtwisted contact structures on closed 3-manifolds coincides with their homotopy classification as tangent plane fields. The classification of tight contact structures is still open.
} 


\section{Part V}

\section{Compatible Almost Complex Structures}

The fact that any symplectic manifold possesses almost complex structures, and even so in a compatible sense, establishes a link from symplectic geometry to complex geometry, and is the point of departure for the modern technique of counting pseudo-holomorphic curves, as first proposed by Gromov [49].

\section{Almost Complex Structures}

\subsection{Three Geometries}

1. Symplectic geometry:

geometry of a closed nondegenerate skew-symmetric bilinear form.

2. Riemannian geometry:

geometry of a positive-definite symmetric bilinear map.

3. Complex geometry:

geometry of a linear map with square -1 .

Example. The euclidean space $\mathbb{R}^{2 n}$ with the standard linear coordinates $\left(x_{1}, \ldots, x_{n}, y_{1}, \ldots, y_{n}\right)$ has standard structures:

$$
\begin{aligned}
& \omega_{0}=\sum d x_{j} \wedge d y_{j}, \quad \text { standard symplectic structure; } \\
& g_{0}=\langle\cdot, \cdot, \quad \text { standard inner product; and }
\end{aligned}
$$

if we identify $\mathbb{R}^{2 n}$ with $\mathbb{C}^{n}$ with coordinates $z_{j}=x_{j}+\sqrt{-1} y_{j}$, then multiplication by $\sqrt{-1}$ induces a constant linear map $J_{0}$ on the tangent spaces of $\mathbb{R}^{2 n}$ :

$$
J_{0}\left(\frac{\partial}{\partial x_{j}}\right)=\frac{\partial}{\partial y_{j}}, \quad J_{0}\left(\frac{\partial}{\partial y_{j}}\right)=-\frac{\partial}{\partial x_{j}}
$$

with $J_{0}^{2}=-$ Id. Relative to the basis $\frac{\partial}{\partial x_{1}}, \ldots, \frac{\partial}{\partial x_{n}}, \frac{\partial}{\partial y_{1}}, \ldots, \frac{\partial}{\partial y_{n}}$, the maps $J_{0}, \omega_{0}$ and $g_{0}$ are represented by

$$
\begin{aligned}
J_{0}(u) & =\left(\begin{array}{cc}
0 & -\mathrm{Id} \\
\mathrm{Id} & 0
\end{array}\right) u \\
\omega_{0}(u, v) & =v^{t}\left(\begin{array}{cc}
0 & -\mathrm{Id} \\
\mathrm{Id} & 0
\end{array}\right) u \\
g_{0}(u, v) & =v^{t} u
\end{aligned}
$$


where $u, v \in \mathbb{R}^{2 n}$ and $v^{t}$ is the transpose of $v$. The following compatibility relation holds:

$$
\omega_{0}(u, v)=g_{0}\left(J_{0}(u), v\right)
$$

\subsection{Complex Structures on Vector Spaces}

Definition 12.1 Let $V$ be a vector space. A complex structure on $V$ is a linear map:

$$
J: V \rightarrow V \quad \text { with } \quad J^{2}=-\mathrm{Id} .
$$

The pair $(V, J)$ is called a complex vector space.

A complex structure $J$ is equivalent to a structure of vector space over $\mathbb{C}$ if we identify the map $J$ with multiplication by $\sqrt{-1}$.

Definition 12.2 Let $(V, \Omega)$ be a symplectic vector space. A complex structure $J$ on $V$ is said to be compatible (with $\Omega$, or $\Omega$-compatible) if

$$
G_{J}(u, v):=\Omega(u, J v), \quad \forall u, v \in V, \text { is a positive inner product on } V .
$$

That is,

$$
J \text { is } \Omega \text {-compatible } \Longleftrightarrow \begin{cases}\Omega(J u, J v)=\Omega(u, v) & \text { [symplectomorphism] } \\ \Omega(u, J u)>0, \quad \forall u \neq 0 & \text { [taming condition] }\end{cases}
$$

Compatible complex structures always exist on symplectic vector spaces:

Proposition 12.3 Let $(V, \Omega)$ be a symplectic vector space. Then there is a compatible complex structure $J$ on $V$.

Proof. Choose a positive inner product $G$ on $V$. Since $\Omega$ and $G$ are nondegenerate,

$$
\left.\begin{array}{rl}
u \in V & \longmapsto \Omega(u, \cdot) \in V^{*} \\
w \in V & \longmapsto G(w, \cdot) \in V^{*}
\end{array}\right\} \text { are isomorphisms between } V \text { and } V^{*} .
$$

Hence, $\Omega(u, v)=G(A u, v)$ for some linear map $A: V \rightarrow V$. This map $A$ is skew-symmetric because

$$
\begin{aligned}
G\left(A^{*} u, v\right) & =G(u, A v)=G(A v, u) \\
& =\Omega(v, u)=-\Omega(u, v)=G(-A u, v) .
\end{aligned}
$$

Also:

- $A A^{*}$ is symmetric: $\left(A A^{*}\right)^{*}=A A^{*}$.

- $A A^{*}$ is positive: $G\left(A A^{*} u, u\right)=G\left(A^{*} u, A^{*} u\right)>0$, for $u \neq 0$. 
These properties imply that $A A^{*}$ diagonalizes with positive eigenvalues $\lambda_{i}$,

$$
A A^{*}=B \operatorname{diag}\left(\lambda_{1}, \ldots, \lambda_{2 n}\right) B^{-1} .
$$

We may hence define an arbitrary real power of $A A^{*}$ by rescaling the eigenspaces, in particular,

$$
\sqrt{A A^{*}}:=B \operatorname{diag}\left(\sqrt{\lambda_{1}}, \ldots, \sqrt{\lambda_{2 n}}\right) B^{-1} .
$$

Then $\sqrt{A A^{*}}$ is symmetric and positive-definite. Let

$$
J=\left(\sqrt{A A^{*}}\right)^{-1} A .
$$

The factorization $A=\sqrt{A A^{*}} J$ is called the polar decomposition of $A$. Since $A$ commutes with $\sqrt{A A^{*}}, J$ commutes with $\sqrt{A A^{*}}$. Check that $J$ is orthogonal, $J J^{*}=\mathrm{Id}$, as well as skew-adjoint, $J^{*}=-J$, and hence it is a complex structure on $V$ :

$$
J^{2}=-J J^{*}=-\mathrm{Id} .
$$

Compatibility:

$$
\begin{aligned}
\Omega(J u, J v) & =G(A J u, J v)=G(J A u, J v)=G(A u, v) \\
& =\Omega(u, v) \\
\Omega(u, J u) & =G(A u, J u)=G(-J A u, u) \\
& =G\left(\sqrt{A A^{*}} u, u\right)>0, \quad \text { for } u \neq 0
\end{aligned}
$$

Therefore, $J$ is a compatible complex structure on $V$.

As indicated in the proof, in general, the positive inner product defined by

$$
\Omega(u, J v)=G\left(\sqrt{A A^{*}} u, v\right) \text { is different from } G(u, v) .
$$

\section{Remarks.}

1. This construction is canonical after an initial choice of $G$. To see this, notice that $\sqrt{A A^{*}}$ does not depend on the choice of $B$ nor of the ordering of the eigenvalues in $\operatorname{diag}\left(\sqrt{\lambda_{1}}, \ldots, \sqrt{\lambda_{2 n}}\right)$. The linear transformation $\sqrt{A A^{*}}$ is completely determined by its effect on each eigenspace of $A A^{*}$ : on the eigenspace corresponding to the eigenvalue $\lambda_{k}$, the map $\sqrt{A A^{*}}$ is defined to be multiplication by $\sqrt{\lambda_{k}}$.

2. If $\left(V_{t}, \Omega_{t}\right)$ is a family of symplectic vector spaces with a family $G_{t}$ of positive inner products, all depending smoothly on a real parameter $t$, then, adapting the proof of the previous proposition, we can show that there is a smooth family $J_{t}$ of compatible complex structures on $V_{t}$.

3. To check just the existence of compatible complex structures on a symplectic vector space $(V, \Omega)$, we could also proceed as follows. Given a symplectic basis $e_{1}, \ldots, e_{n}, f_{1}, \ldots, f_{n}$ (i.e., $\Omega\left(e_{i}, e_{j}\right)=\Omega\left(f_{i}, f_{j}\right)=0$ and $\left.\Omega\left(e_{i}, f_{j}\right)=\delta_{i j}\right)$, one can define $J e_{j}=f_{j}$ and $J f_{j}=-e_{j}$. This is a compatible complex 
structure on $(V, \Omega)$. Moreover, given $\Omega$ and $J$ compatible on $V$, there exists a symplectic basis of $V$ of the form:

$$
e_{1}, \ldots, e_{n}, f_{1}=J e_{1}, \ldots, f_{n}=J e_{n} .
$$

The proof is part of Homework 8.

4. Conversely, given $(V, J)$, there is always a symplectic structure $\Omega$ such that $J$ is $\Omega$-compatible: pick any positive inner product $G$ such that $J^{*}=-J$ and take $\Omega(u, v)=G(J u, v)$.

\subsection{Compatible Structures}

Definition 12.4 An almost complex structure on a manifold $M$ is a smooth field of complex structures on the tangent spaces:

$$
x \longmapsto J_{x}: T_{x} M \rightarrow T_{x} M \quad \text { linear, and } \quad J_{x}^{2}=-\mathrm{Id} .
$$

The pair $(M, J)$ is then called an almost complex manifold.

Definition 12.5 Let $(M, \omega)$ be a symplectic manifold. An almost complex structure $J$ on $M$ is called compatible (with $\omega$ or $\omega$-compatible) if the assignment

$$
\begin{array}{r}
x \longmapsto \quad g_{x}: T_{x} M \times T_{x} M \rightarrow \mathbb{R} \\
g_{x}(u, v):=\omega_{x}\left(u, J_{x} v\right)
\end{array}
$$

is a riemannian metric on $M$.

For a manifold $M$,

$\omega$ is a symplectic form $\quad \Longrightarrow x \longmapsto \omega_{x}: T_{x} M \times T_{x} M \rightarrow \mathbb{R}$ is bilinear, nondegenerate, skew-symmetric;

$g$ is a riemannian metric $\quad \Longrightarrow \quad x \longmapsto g_{x}: T_{x} M \times T_{x} M \rightarrow \mathbb{R}$ is a positive inner product;

$J$ almost complex structure $\Longrightarrow x \longmapsto J_{x}: T_{x} M \rightarrow T_{x} M$ is linear and $J^{2}=-\mathrm{Id}$.

The triple $(\omega, g, J)$ is called a compatible triple when $g(\cdot, \cdot)=\omega(\cdot, J \cdot)$.

Proposition 12.6 Let $(M, \omega)$ be a symplectic manifold, and $g$ a riemannian metric on $M$. Then there exists a canonical almost complex structure $J$ on $M$ which is compatible.

Proof. The polar decomposition is canonical (after a choice of metric), hence this construction of $J$ on $M$ is smooth; cf. Remark 2 of the previous section.

Remark. In general, $g_{J}(\cdot, \cdot):=\omega(\cdot, J \cdot) \neq g(\cdot, \cdot)$.

Since riemannian metrics always exist, we conclude: 
Corollary 12.7 Any symplectic manifold has compatible almost complex structures.

- How different can compatible almost complex structures be?

Proposition 12.8 Let $(M, \omega)$ be a symplectic manifold, and $J_{0}, J_{1}$ two almost complex structures compatible with $\omega$. Then there is a smooth family $J_{t}, 0 \leq t \leq 1$, of compatible almost complex structures joining $J_{0}$ to $J_{1}$.

Proof. By compatibility, we get

$$
\left.\begin{array}{l}
\omega, J_{0} \rightsquigarrow g_{0}(\cdot, \cdot)=\omega\left(\cdot, J_{0} \cdot\right) \\
\omega, J_{1} \rightsquigarrow g_{1}(\cdot, \cdot)=\omega\left(\cdot, J_{1} \cdot\right)
\end{array}\right\} \quad \text { two riemannian metrics on } M .
$$

Their convex combinations

$$
g_{t}(\cdot, \cdot)=(1-t) g_{0}(\cdot, \cdot)+t g_{1}(\cdot, \cdot), \quad 0 \leq t \leq 1,
$$

form a smooth family of riemannian metrics. Apply the polar decomposition to $\left(\omega, g_{t}\right)$ to obtain a smooth family of $J_{t}$ 's joining $J_{0}$ to $J_{1}$.

Corollary 12.9 The set of all compatible almost complex structures on a symplectic manifold is path-connected. 


\section{Homework 8: Compatible Linear Structures}

1. Let $\Omega(V)$ and $J(V)$ be the spaces of symplectic forms and complex structures on the vector space $V$, respectively. Take $\Omega \in \Omega(V)$ and $J \in J(V)$. Let $\mathrm{GL}(V)$ be the group of all isomorphisms of $V$, let $\operatorname{Sp}(V, \Omega)$ be the group of symplectomorphisms of $(V, \Omega)$, and let $\operatorname{GL}(V, J)$ be the group of complex isomorphisms of $(V, J)$.

Show that

$$
\Omega(V) \simeq \mathrm{GL}(V) / \mathrm{Sp}(V, \Omega) \quad \text { and } \quad J(V) \simeq \mathrm{GL}(V) / \mathrm{GL}(V, J) .
$$

Hint: The group $\operatorname{GL}(V)$ acts on $\Omega(V)$ by pullback. What is the stabilizer of a given $\Omega$ ?

2. Let $\left(\mathbb{R}^{2 n}, \Omega_{0}\right)$ be the standard $2 n$-dimensional symplectic euclidean space.

The symplectic linear group is the group of all linear transformations of $\mathbb{R}^{2 n}$ which preserve the symplectic structure:

$$
\operatorname{Sp}(2 n):=\left\{A \in \mathrm{GL}(2 n ; \mathbb{R}) \mid \Omega_{0}(A u, A v)=\Omega_{0}(u, v) \text { for all } u, v \in \mathbb{R}^{2 n}\right\} .
$$

Identifying the complex $n \times n$ matrix $X+i Y$ with the real $2 n \times 2 n$ matrix $\left(\begin{array}{cc}X & -Y \\ Y & X\end{array}\right)$, consider the following subgroups of $\mathrm{GL}(2 n ; \mathbb{R})$ :

$$
\mathrm{Sp}(2 n), \mathrm{O}(2 n), \mathrm{GL}(n ; \mathbb{C}) \text { and } \mathrm{U}(n)
$$

Show that the intersection of any two of them is $\mathrm{U}(n)$. (From [83, p.41].)

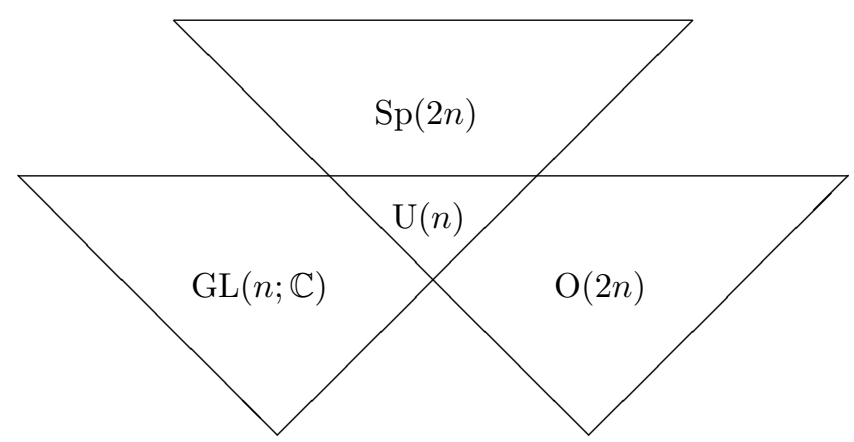


3. Let $(V, \Omega)$ be a symplectic vector space of dimension $2 n$, and let $J: V \rightarrow V$, $J^{2}=-\mathrm{Id}$, be a complex structure on $V$.

(a) Prove that, if $J$ is $\Omega$-compatible and $L$ is a lagrangian subspace of $(V, \Omega)$, then $J L$ is also lagrangian and $J L=L^{\perp}$, where $\perp$ denotes orthogonality with respect to the positive inner product $G_{J}(u, v)=\Omega(u, J v)$.

(b) Deduce that $J$ is $\Omega$-compatible if and only if there exists a symplectic basis for $V$ of the form

$$
e_{1}, e_{2}, \ldots, e_{n}, f_{1}=J e_{1}, f_{2}=J e_{2}, \ldots, f_{n}=J e_{n}
$$

where $\Omega\left(e_{i}, e_{j}\right)=\Omega\left(f_{i}, f_{j}\right)=0$ and $\Omega\left(e_{i}, f_{j}\right)=\delta_{i j}$. 


\section{Compatible Triples}

\subsection{Compatibility}

Let $(M, \omega)$ be a symplectic manifold. As shown in the previous lecture, compatible almost complex structures always exist on $(M, \omega)$. We also showed that the set of all compatible almost complex structures on $(M, \omega)$ is path-connected. In fact, the set of all compatible almost complex structures is even contractible. (This is important for defining invariants.) Let $\mathcal{J}\left(T_{x} M, \omega_{x}\right)$ be the set of all compatible complex structures on $\left(T_{x} M, \omega_{x}\right)$ for $x \in M$.

Proposition 13.1 The set $\mathcal{J}\left(T_{x} M, \omega_{x}\right)$ is contractible, i.e., there exists a homotopy

$$
h_{t}: \mathcal{J}\left(T_{x} M, \omega_{x}\right) \longrightarrow \mathcal{J}\left(T_{x} M, \omega_{x}\right), \quad 0 \leq t \leq 1,
$$

starting at the identity $h_{0}=\mathrm{Id}$,

finishing at a trivial map $h_{1}: \mathcal{J}\left(T_{x} M, \omega_{x}\right) \rightarrow\left\{J_{0}\right\}$,

and fixing $J_{0}$ (i.e., $h_{t}\left(J_{0}\right)=J_{0}, \forall t$ ) for some $J_{0} \in \mathcal{J}\left(T_{x} M, \omega_{x}\right)$.

Proof. Homework 9.

Consider the fiber bundle $\mathcal{J} \rightarrow M$ with fiber

$$
\mathcal{J}_{x}:=\mathcal{J}\left(T_{x} M, \omega_{x}\right) \quad \text { over } x \in M
$$

A compatible almost complex structure $J$ on $(M, \omega)$ is a section of $\mathcal{J}$. The space of sections of $\mathcal{J}$ is contractible because the fibers are contractible.

\section{Remarks.}

- We never used the closedness of $\omega$ to construct compatible almost complex structures. The construction holds for an almost symplectic manifold $(M, \omega)$, that is, a pair of a manifold $M$ and a nondegenerate 2-form $\omega$, not necessarily closed.

- Similarly, we could define a symplectic vector bundle to be a vector bundle $E \rightarrow M$ over a manifold $M$ equipped with a smooth field $\omega$ of fiberwise nondegenerate skew-symmetric bilinear maps

$$
\omega_{x}: E_{x} \times E_{x} \longrightarrow \mathbb{R}
$$

The existence of such a field $\omega$ is equivalent to being able to reduce the structure group of the bundle from the general linear group to the linear symplectic group. As a consequence of our discussion, a symplectic vector bundle is always a complex vector bundle, and vice-versa. 


\subsection{Triple of Structures}

If $(\omega, J, g)$ is a compatible triple, then any one of $\omega, J$ or $g$ can be written in terms of the other two:

$$
\begin{aligned}
g(u, v) & =\omega(u, J v) \\
\omega(u, v) & =g(J u, v) \\
J(u) & =\widetilde{g}^{-1}(\widetilde{\omega}(u))
\end{aligned}
$$

where

$$
\begin{aligned}
& \widetilde{\omega}: T M \longrightarrow T^{*} M \quad u \longmapsto \omega(u, \cdot) \\
& \widetilde{g}: T M \longrightarrow T^{*} M \quad u \longmapsto g(u, \cdot)
\end{aligned}
$$

are the linear isomorphisms induced by the bilinear forms $\omega$ and $g$.

The relations among $\omega, J$ and $g$ can be summarized in the following table. The last column lists differential equations these structures are usually asked to satisfy.

$$
\begin{array}{llll}
\text { Data } & \text { Condition/Technique } & \text { Consequence } & \text { Question } \\
\omega, J & \begin{array}{l}
\omega(J u, J v)=\omega(u, v) \\
\omega(u, J u)>0, u \neq 0
\end{array} & \begin{array}{l}
g(u, v):=\omega(u, J v) \\
\text { is positive inner product }
\end{array} & \text { ( } g \text { flat?) } \\
g, J & \begin{array}{l}
g(J u, J v)=g(u, v) \\
(\text { i.e., } J \text { is orthogonal })
\end{array} & \begin{array}{l}
\omega(u, v):=g(J u, v) \\
\text { is nondeg., skew-symm. }
\end{array} & \text { w closed? } \\
\omega, g & \text { polar decomposition } \rightsquigarrow & J \text { almost complex str. } & J \text { integrable? }
\end{array}
$$

An almost complex structure $J$ on a manifold $M$ is called integrable if and only if $J$ is induced by a structure of complex manifold on $M$. In Lecture 15 we will discuss tests to check whether a given $J$ is integrable.

\subsection{First Consequences}

Proposition 13.2 Let $(M, J)$ be an almost complex manifold. Suppose that $J$ is compatible with two symplectic structures $\omega_{0}, \omega_{1}$ Then $\omega_{0}, \omega_{1}$ are deformationequivalent, that is, there exists a smooth family $\omega_{t}, 0 \leq t \leq 1$, of symplectic forms joining $\omega_{0}$ to $\omega_{1}$.

Proof. Take $\omega_{t}=(1-t) \omega_{0}+t \omega_{1}, 0 \leq t \leq 1$. Then:

- $\omega_{t}$ is closed.

- $\omega_{t}$ is nondegenerate, since

$$
g_{t}(\cdot, \cdot):=\omega_{t}(\cdot, J \cdot)=(1-t) g_{0}(\cdot, \cdot)+t g_{1}(\cdot, \cdot)
$$

is positive, hence nondegenerate. 
Remark. The converse of this proposition is not true. A counterexample is provided by the following family in $\mathbb{R}^{4}$ :

$\omega_{t}=\cos \pi t d x_{1} d y_{1}+\sin \pi t d x_{1} d y_{2}+\sin \pi t d y_{1} d x_{2}+\cos \pi t d x_{2} d y_{2}, 0 \leq t \leq 1$.

There is no $J$ in $\mathbb{R}^{4}$ compatible with both $\omega_{0}$ and $\omega_{1}$.

Definition 13.3 A submanifold $X$ of an almost complex manifold $(M, J)$ is an almost complex submanifold when $J(T X) \subseteq T X$, i.e., for all $x \in X, v \in T_{x} X$, we have $J_{x} v \in T_{x} X$.

Proposition 13.4 Let $(M, \omega)$ be a symplectic manifold equipped with a compatible almost complex structure $J$. Then any almost complex submanifold $X$ of $(M, J)$ is a symplectic submanifold of $(M, \omega)$.

Proof. Let $i: X \hookrightarrow M$ be the inclusion. Then $i^{*} \omega$ is a closed 2-form on $X$. Nondegeneracy:

$$
\omega_{x}(u, v)=g_{x}\left(J_{x} u, v\right), \quad \forall x \in X, \forall u, v \in T_{x} X .
$$

Since $\left.g_{x}\right|_{T_{x} X}$ is nondegenerate, so is $\left.\omega_{x}\right|_{T_{x} X}$. Hence, $i^{*} \omega$ is symplectic.

- When is an almost complex manifold a complex manifold? See Lecture 15.

\section{Examples.}

$S^{2}$ is an almost complex manifold and it is a complex manifold.

$S^{4}$ is not an almost complex manifold (proved by Ehresmann and Hopf).

$S^{6}$ is almost complex and it is not yet known whether it is complex.

$S^{8}$ and higher spheres are not almost complex manifolds. 


\section{Homework 9: Contractibility}

The following proof illustrates in a geometric way the relation between lagrangian subspaces, complex structures and inner products; from [11, p.45].

Let $(V, \Omega)$ be a symplectic vector space, and let $\mathcal{J}(V, \Omega)$ be the set of all complex structures on $(V, \Omega)$ which are $\Omega$-compatible; i.e., given a complex structure $J$ on $V$ we have

$$
J \in \mathcal{J}(V, \Omega) \Longleftrightarrow G_{J}(\cdot, \cdot):=\Omega(\cdot, J \cdot) \text { is a positive inner product on } V .
$$

Fix a lagrangian subspace $L_{0}$ of $(V, \Omega)$. Let $\mathcal{L}\left(V, \Omega, L_{0}\right)$ be the space of all lagrangian subspaces of $(V, \Omega)$ which intersect $L_{0}$ transversally. Let $\mathcal{G}\left(L_{0}\right)$ be the space of all positive inner products on $L_{0}$.

Consider the map

$$
\begin{aligned}
\Psi: \mathcal{J}(V, \Omega) & \rightarrow \mathcal{L}\left(V, \Omega, L_{0}\right) \times \mathcal{G}\left(L_{0}\right) \\
J & \mapsto\left(J L_{0},\left.G_{J}\right|_{L_{0}}\right)
\end{aligned}
$$

Show that:

1. $\Psi$ is well-defined.

2. $\Psi$ is a bijection.

Hint: Given $(L, G) \in \mathcal{L}\left(V, \Omega, L_{0}\right) \times \mathcal{G}\left(L_{0}\right)$, define $J$ in the following manner: For $v \in L_{0}, v^{\perp}=\left\{u \in L_{0} \mid G(u, v)=0\right\}$ is a $(n-1)$-dimensional space of $L_{0}$; its symplectic orthogonal $\left(v^{\perp}\right)^{\Omega}$ is $(n+1)$-dimensional. Check that $\left(v^{\perp}\right)^{\Omega} \cap L$ is 1-dimensional. Let $J v$ be the unique vector in this line such that $\Omega(v, J v)=1$. Check that, if we take $v$ 's in some $G$-orthonormal basis of $L_{0}$, this defines the required element of $\mathcal{J}(V, \Omega)$.

3. $\mathcal{L}\left(V, \Omega, L_{0}\right)$ is contractible.

Hint: Prove that $\mathcal{L}\left(V, \Omega, L_{0}\right)$ can be identified with the vector space of all symmetric $n \times n$ matrices. Notice that any $n$-dimensional subspace $L$ of $V$ which is transversal to $L_{0}$ is the graph of a linear map $S: J L_{0} \rightarrow L_{0}$, i.e.,

$$
\begin{aligned}
L & =\text { span of }\left\{J e_{1}+S J e_{1}, \ldots, J e_{n}+S J e_{n}\right\} \\
\text { when } \quad L_{0} & =\text { span of }\left\{e_{1}, \ldots, e_{n}\right\} .
\end{aligned}
$$

4. $\mathcal{G}\left(L_{0}\right)$ is contractible.

Hint: $\mathcal{G}\left(L_{0}\right)$ is even convex.

Conclude that $\mathcal{J}(V, \Omega)$ is contractible. 


\section{Dolbeault Theory}

\section{$14.1 \quad$ Splittings}

Let $(M, J)$ be an almost complex manifold. The complexified tangent bundle of $M$ is the bundle

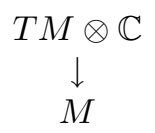

with fiber $(T M \otimes \mathbb{C})_{p}=T_{p} M \otimes \mathbb{C}$ at $p \in M$. If

$$
\begin{array}{ll}
T_{p} M & \text { is a } 2 n \text {-dimensional vector space over } \mathbb{R}, \text { then } \\
T_{p} M \otimes \mathbb{C} & \text { is a } 2 n \text {-dimensional vector space over } \mathbb{C} .
\end{array}
$$

We may extend $J$ linearly to $T M \otimes \mathbb{C}$ :

$$
J(v \otimes c)=J v \otimes c, \quad v \in T M, \quad c \in \mathbb{C} .
$$

Since $J^{2}=-\mathrm{Id}$, on the complex vector space $(T M \otimes \mathbb{C})_{p}$, the linear map $J_{p}$ has eigenvalues $\pm i$. Let

$$
\begin{aligned}
T_{1,0} & =\{v \in T M \otimes \mathbb{C} \mid J v=+i v\}=(+i) \text {-eigenspace of } J \\
& =\{v \otimes 1-J v \otimes i \mid v \in T M\} \\
& =(J \text {-)holomorphic tangent vectors } ; \\
T_{0,1} & =\{v \in T M \otimes \mathbb{C} \mid J v=-i v\}=(-i) \text {-eigenspace of } J \\
& =\{v \otimes 1+J v \otimes i \mid v \in T M\} \\
& =(J \text {-)anti-holomorphic tangent vectors } .
\end{aligned}
$$

Since

$$
\begin{aligned}
\pi_{1,0}: T M & \longrightarrow T_{1,0} \\
v & \longmapsto \frac{1}{2}(v \otimes 1-J v \otimes i)
\end{aligned}
$$

is a (real) bundle isomorphism such that $\pi_{1,0} \circ J=i \pi_{1,0}$, and

$$
\begin{aligned}
\pi_{0,1}: T M & \longrightarrow T_{0,1} \\
v & \longmapsto \frac{1}{2}(v \otimes 1+J v \otimes i)
\end{aligned}
$$

is also a (real) bundle isomorphism such that $\pi_{0,1} \circ J=-i \pi_{0,1}$, we conclude that we have isomorphisms of complex vector bundles

$$
(T M, J) \simeq T_{1,0} \simeq \overline{T_{0,1}},
$$

where $\overline{T_{0,1}}$ denotes the complex conjugate bundle of $T_{0,1}$. Extending $\pi_{1,0}$ and $\pi_{0,1}$ to projections of $T M \otimes \mathbb{C}$, we obtain an isomorphism

$$
\left(\pi_{1,0}, \pi_{0,1}\right): T M \otimes \mathbb{C} \stackrel{\simeq}{\longrightarrow} T_{1,0} \oplus T_{0,1} .
$$


Similarly, the complexified cotangent bundle splits as

$$
\left(\pi^{1,0}, \pi^{0,1}\right): T^{*} M \otimes \mathbb{C} \stackrel{\simeq}{\longrightarrow} T^{1,0} \oplus T^{0,1}
$$

where

$$
\begin{aligned}
T^{1,0} & =\left(T_{1,0}\right)^{*}=\left\{\eta \in T^{*} \otimes \mathbb{C} \mid \eta(J \omega)=i \eta(\omega), \forall \omega \in T M \otimes \mathbb{C}\right\} \\
& =\left\{\xi \otimes 1-(\xi \circ J) \otimes i \mid \xi \in T^{*} M\right\} \\
& =\text { complex-linear cotangent vectors }, \\
T^{0,1} & =\left(T_{0,1}\right)^{*}=\left\{\eta \in T^{*} \otimes \mathbb{C} \mid \eta(J \omega)=-i \eta(\omega), \forall \omega \in T M \otimes \mathbb{C}\right\} \\
& =\left\{\xi \otimes 1+(\xi \circ J) \otimes i \mid \xi \in T^{*} M\right\} \\
& =\text { complex-antilinear cotangent vectors },
\end{aligned}
$$

and $\pi^{1,0}, \pi^{0,1}$ are the two natural projections

$$
\begin{aligned}
\pi^{1,0}: T^{*} M \otimes \mathbb{C} & \longrightarrow T^{1,0} \\
\eta & \longmapsto \eta^{1,0}:=\frac{1}{2}(\eta-i \eta \circ J) ; \\
\pi^{0,1}: T^{*} M \otimes \mathbb{C} & \longrightarrow T^{0,1} \\
\eta & \longmapsto \eta^{0,1}:=\frac{1}{2}(\eta+i \eta \circ J) .
\end{aligned}
$$

\subsection{Forms of Type $(\ell, m)$}

For an almost complex manifold $(M, J)$, let

$$
\begin{aligned}
\Omega^{k}(M ; \mathbb{C}) & :=\text { sections of } \Lambda^{k}\left(T^{*} M \otimes \mathbb{C}\right) \\
& =\text { complex-valued k-forms on } M, \text { where } \\
\Lambda^{k}\left(T^{*} M \otimes \mathbb{C}\right) & :=\Lambda^{k}\left(T^{1,0} \oplus T^{0,1}\right) \\
& =\oplus_{\ell+m=k} \underbrace{\left(\Lambda^{\ell} T^{1,0}\right) \wedge\left(\Lambda^{m} T^{0,1}\right)}_{\Lambda^{\ell, m}(\text { definition })} \\
& =\oplus_{\ell+m=k} \Lambda^{\ell, m} .
\end{aligned}
$$

In particular, $\Lambda^{1,0}=T^{1,0}$ and $\Lambda^{0,1}=T^{0,1}$.

Definition 14.1 The differential forms of type $(\ell, \boldsymbol{m})$ on $(M, J)$ are the sections of $\Lambda^{\ell, m}$ :

$$
\Omega^{\ell, m}:=\text { sections of } \Lambda^{\ell, m} \text {. }
$$

Then

$$
\Omega^{k}(M ; \mathbb{C})=\oplus_{\ell+m=k} \Omega^{\ell, m} .
$$

Let $\pi^{\ell, m}: \Lambda^{k}\left(T^{*} M \otimes \mathbb{C}\right) \rightarrow \Lambda^{\ell, m}$ be the projection map, where $\ell+m=k$. The usual exterior derivative $d$ composed with two of these projections induces differential operators $\partial$ and $\bar{\partial}$ on forms of type $(\ell, m)$ :

$$
\begin{aligned}
& \partial:=\pi^{\ell+1, m} \circ d: \Omega^{\ell, m}(M) \longrightarrow \Omega^{\ell+1, m}(M) \\
& \bar{\partial}:=\pi^{\ell, m+1} \circ d: \Omega^{\ell, m}(M) \longrightarrow \Omega^{\ell, m+1}(M) .
\end{aligned}
$$


If $\beta \in \Omega^{\ell, m}(M)$, with $k=\ell+m$, then $d \beta \in \Omega^{k+1}(M ; \mathbb{C})$ :

$$
d \beta=\sum_{r+s=k+1} \pi^{r, s} d \beta=\pi^{k+1,0} d \beta+\cdots+\partial \beta+\bar{\partial} \beta+\cdots+\pi^{0, k+1} d \beta .
$$

\subsection{J-Holomorphic Functions}

Let $f: M \rightarrow \mathbb{C}$ be a smooth complex-valued function on $M$. The exterior derivative $d$ extends linearly to $\mathbb{C}$-valued functions as $d f=d(\operatorname{Re} f)+i d(\operatorname{Im} f)$.

Definition 14.2 A function $f$ is $\left(\boldsymbol{J}\right.$-)holomorphic at $x \in M$ if $d f_{p}$ is complex linear, i.e., $d f_{p} \circ J=i d f_{p}$. A function $f$ is $(J$-)holomorphic if it is holomorphic at all $p \in M$.

Exercise. Show that

$$
d f_{p} \circ J=i d f_{p} \quad \Longleftrightarrow \quad d f_{p} \in T_{p}^{1,0} \quad \Longleftrightarrow \quad \pi_{p}^{0,1} d f_{p}=0
$$

Definition 14.3 A function $f$ is $\left(J\right.$-)anti-holomorphic at $p \in M$ if $d f_{p}$ is complex antilinear, i.e., $d f_{p} \circ J=-i d f_{p}$.

Exercise.

$$
\begin{aligned}
d f_{p} \circ J=-i d f_{p} & \Longleftrightarrow d f_{p} \in T_{p}^{0,1} \Longleftrightarrow \pi_{p}^{1,0} d f_{p}=0 \\
& \Longleftrightarrow d \bar{f}_{p} \in T_{p}^{1,0} \Longleftrightarrow \pi_{p}^{0,1} d \bar{f}_{p}=0 \\
& \Longleftrightarrow \bar{f} \text { is holomorphic at } p \in M .
\end{aligned}
$$

Definition 14.4 On functions, $d=\partial+\bar{\partial}$, where

$$
\partial:=\pi^{1,0} \circ d \quad \text { and } \quad \bar{\partial}:=\pi^{0,1} \circ d .
$$

Then

$$
\begin{aligned}
f \text { is holomorphic } & \Longleftrightarrow \bar{\partial} f=0, \\
f \text { is anti-holomorphic } & \Longleftrightarrow \partial f=0 .
\end{aligned}
$$

- What about higher differential forms? 


\subsection{Dolbeault Cohomology}

Suppose that $d=\partial+\bar{\partial}$, i.e.,

$$
d \beta=\underbrace{\partial \beta}_{\in \Omega^{\ell+1, m}}+\underbrace{\bar{\partial} \beta}_{\in \Omega^{\ell, m+1}}, \quad \forall \beta \in \Omega^{\ell, m} .
$$

Then, for any form $\beta \in \Omega^{\ell, m}$,

$$
0=d^{2} \beta=\underbrace{\partial^{2} \beta}_{\in \Omega^{\ell+2, m}}+\underbrace{\partial \bar{\partial} \beta+\bar{\partial} \partial \beta}_{\in \Omega^{\ell+1, m+1}}+\underbrace{\bar{\partial}^{2} \beta}_{\in \Omega^{\ell, m+2}},
$$

which implies

$$
\left\{\begin{array}{l}
\bar{\partial}^{2}=0 \\
\partial \bar{\partial}+\bar{\partial} \partial=0 \\
\partial^{2}=0
\end{array}\right.
$$

Since $\bar{\partial}^{2}=0$, the chain

$$
0 \longrightarrow \Omega^{\ell, 0} \stackrel{\bar{\partial}}{\longrightarrow} \Omega^{\ell, 1} \stackrel{\bar{\partial}}{\longrightarrow} \Omega^{\ell, 2} \stackrel{\bar{\partial}}{\longrightarrow} \cdots
$$

is a differential complex; its cohomology groups

$$
H_{\text {Dolbeault }}^{\ell, m}(M):=\frac{\operatorname{ker} \bar{\partial}: \Omega^{\ell, m} \longrightarrow \Omega^{\ell, m+1}}{\operatorname{im} \bar{\partial}: \Omega^{\ell, m-1} \longrightarrow \Omega^{\ell, m}}
$$

are called the Dolbeault cohomology groups.

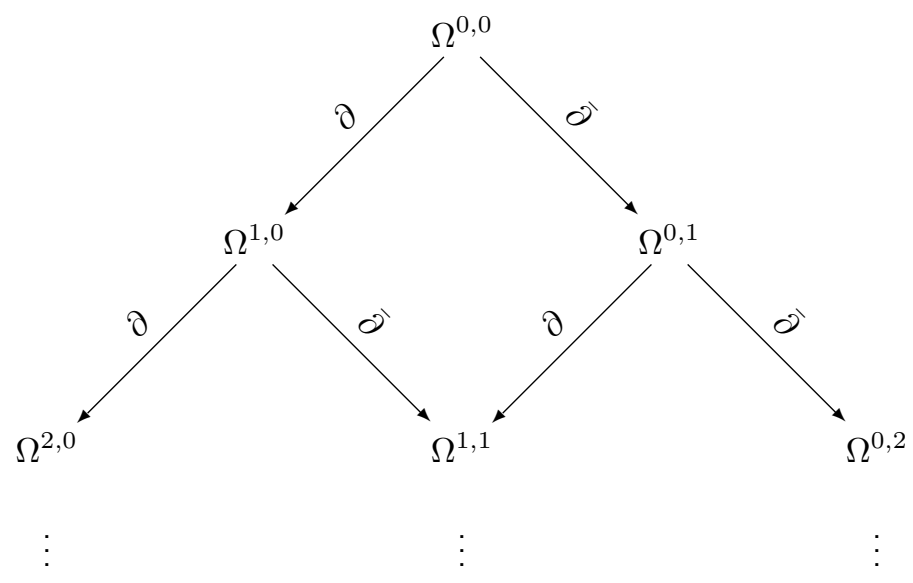

- When is $d=\partial+\bar{\partial}$ ? See the next lecture. 


\section{Homework 10: Integrability}

This set of problems is from [11, p.46-47].

1. Let $(M, J)$ be an almost complex manifold. Its Nijenhuis tensor $\mathcal{N}$ is:

$$
\mathcal{N}(v, w):=[J v, J w]-J[v, J w]-J[J v, w]-[v, w],
$$

where $v$ and $w$ are vector fields on $M,[\cdot, \cdot]$ is the usual bracket

$$
[v, w] \cdot f:=v \cdot(w \cdot f)-w \cdot(v \cdot f), \text { for } f \in C^{\infty}(M),
$$

and $v \cdot f=d f(v)$.

(a) Check that, if the map $v \mapsto[v, w]$ is complex linear (in the sense that it commutes with $J$ ), then $\mathcal{N} \equiv 0$.

(b) Show that $\mathcal{N}$ is actually a tensor, that is: $\mathcal{N}(v, w)$ at $x \in M$ depends only on the values $v_{x}, w_{x} \in T_{x} M$ and not really on the vector fields $v$ and $w$.

(c) Compute $\mathcal{N}(v, J v)$. Deduce that, if $M$ is a surface, then $\mathcal{N} \equiv 0$.

A theorem of Newlander and Nirenberg [89] states that an almost complex manifold $(M, J)$ is a complex (analytic) manifold if and only if $\mathcal{N} \equiv 0$. Combining (c) with the fact that any orientable surface is symplectic, we conclude that any orientable surface is a complex manifold, a result already known to Gauss.

2. Let $\mathcal{N}$ be as above. For any map $f: \mathbb{R}^{2 n} \rightarrow \mathbb{C}$ and any vector field $v$ on $\mathbb{R}^{2 n}$, we have $v \cdot f=v \cdot\left(f_{1}+i f_{2}\right)=v \cdot f_{1}+i v \cdot f_{2}$, so that $f \mapsto v \cdot f$ is a complex linear map.

(a) Let $\mathbb{R}^{2 n}$ be endowed with an almost complex structure $J$, and suppose that $f$ is a $J$-holomorphic function, that is,

$$
d f \circ J=i d f .
$$

Show that $d f(\mathcal{N}(v, w))=0$ for all vector fields $v, w$.

(b) Suppose that there exist $n J$-holomorphic functions, $f_{1}, \ldots, f_{n}$, on $\mathbb{R}^{2 n}$, which are independent at some point $p$, i.e., the real and imaginary parts of $\left(d f_{1}\right)_{p}, \ldots,\left(d f_{n}\right)_{p}$ form a basis of $T_{p}^{*} \mathbb{R}^{2 n}$. Show that $\mathcal{N}$ vanishes identically at $p$.

(c) Assume that $M$ is a complex manifold and $J$ is its complex structure. Show that $\mathcal{N}$ vanishes identically everywhere on $M$.

In general, an almost complex manifold has no $J$-holomorphic functions at all. On the other hand, it has plenty of $J$-holomorphic curves: maps $f: \mathbb{C} \rightarrow M$ such that $d f \circ i=J \circ d f$. $J$-holomorphic curves, also known as pseudoholomorphic curves, provide a main tool in symplectic topology, as first realized by Gromov [49]. 


\section{Part VI}

\section{Kähler Manifolds}

Kähler geometry lies at the intersection of complex, riemannian and symplectic geometries, and plays a central role in all of these fields. We will start by reviewing complex manifolds. After describing the local normal form for Kähler manifolds (Lecture 16), we conclude with a summary of Hodge theory for compact Kähler manifolds (Lecture 17).

\section{Complex Manifolds}

\subsection{Complex Charts}

Definition 15.1 A complex manifold of (complex) dimension $n$ is a set $M$ with a complete complex atlas

$$
\mathcal{A}=\left\{\left(\mathcal{U}_{\alpha}, \mathcal{V}_{\alpha}, \varphi_{\alpha}\right), \alpha \in \text { index set } I\right\}
$$

where $M=\cup_{\alpha} \mathcal{U}_{\alpha}$, the $\mathcal{V}_{\alpha}$ 's are open subsets of $\mathbb{C}^{n}$, and the maps $\varphi_{\alpha}: \mathcal{U}_{\alpha} \rightarrow \mathcal{V}_{\alpha}$ are such that the transition maps $\psi_{\alpha \beta}$ are biholomorphic as maps on open subsets of $\mathbb{C}^{n}$ :

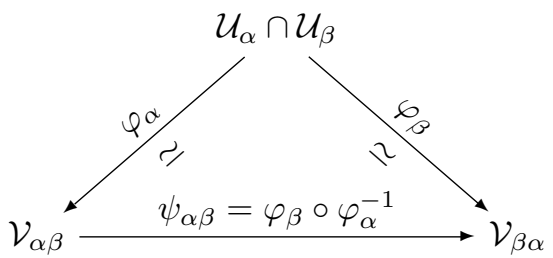

where $\mathcal{V}_{\alpha \beta}=\varphi_{\alpha}\left(\mathcal{U}_{\alpha} \cap \mathcal{U}_{\beta}\right) \subseteq \mathbb{C}^{n}$ and $\mathcal{V}_{\beta \alpha}=\varphi_{\beta}\left(\mathcal{U}_{\alpha} \cap \mathcal{U}_{\beta}\right) \subseteq \mathbb{C}^{n}$. $\psi_{\alpha \beta}$ being biholomorphic means that $\psi_{\alpha \beta}$ is a bijection and that $\psi_{\alpha \beta}$ and $\psi_{\alpha \beta}^{-1}$ are both holomorphic.

Proposition 15.2 Any complex manifold has a canonical almost complex structure.

\section{Proof.}

1) Local definition of $J$ :

Let $(\mathcal{U}, \mathcal{V}, \varphi: \mathcal{U} \rightarrow \mathcal{V})$ be a complex chart for a complex manifold $M$ with $\varphi=\left(z_{1}, \ldots, z_{n}\right)$ written in components relative to complex coordinates $z_{j}=$ $x_{j}+i y_{j}$. At $p \in \mathcal{U}$

$$
T_{p} M=\mathbb{R} \text {-span of }\left\{\left.\frac{\partial}{\partial x_{j}}\right|_{p},\left.\frac{\partial}{\partial y_{j}}\right|_{p}: j=1, \ldots, n\right\} .
$$


Define $J$ over $\mathcal{U}$ by

$$
\begin{aligned}
& J_{p}\left(\left.\frac{\partial}{\partial x_{j}}\right|_{p}\right)=\left.\frac{\partial}{\partial y_{j}}\right|_{p} \quad j=1, \ldots, n . \\
& J_{p}\left(\left.\frac{\partial}{\partial y_{j}}\right|_{p}\right)=-\left.\frac{\partial}{\partial x_{j}}\right|_{p}
\end{aligned}
$$

2) This $J$ is well-defined globally:

If $(\mathcal{U}, \mathcal{V}, \varphi)$ and $\left(\mathcal{U}^{\prime}, \mathcal{V}^{\prime}, \varphi^{\prime}\right)$ are two charts, we need to show that $J=J^{\prime}$ on their overlap.

On $\mathcal{U} \cap \mathcal{U}^{\prime}, \psi \circ \varphi=\varphi^{\prime}$. If $z_{j}=x_{j}+i y_{j}$ and $w_{j}=u_{j}+i v_{j}$ are coordinates on $\mathcal{U}$ and $\mathcal{U}^{\prime}$, respectively, so that $\varphi$ and $\varphi^{\prime}$ can be written in components $\varphi=\left(z_{1}, \ldots, z_{n}\right), \varphi^{\prime}=\left(w_{1}, \ldots, w_{n}\right)$, then $\psi\left(z_{1}, \ldots, z_{n}\right)=\left(w_{1}, \ldots, w_{n}\right)$. Taking the derivative of a composition

$$
\left\{\begin{aligned}
\frac{\partial}{\partial x_{k}} & =\sum_{j}\left(\frac{\partial u_{j}}{\partial x_{k}} \frac{\partial}{\partial u_{j}}+\frac{\partial v_{j}}{\partial x_{k}} \frac{\partial}{\partial v_{j}}\right) \\
\frac{\partial}{\partial y_{k}} & =\sum_{j}\left(\frac{\partial u_{j}}{\partial y_{k}} \frac{\partial}{\partial u_{j}}+\frac{\partial v_{j}}{\partial y_{k}} \frac{\partial}{\partial v_{j}}\right)
\end{aligned}\right.
$$

Since $\psi$ is biholomorphic, each component of $\psi$ satisfies the Cauchy-Riemann equations:

$$
\left\{\begin{array}{l}
\frac{\partial u_{j}}{\partial x_{k}}=\frac{\partial v_{j}}{\partial y_{k}} \\
\frac{\partial u_{j}}{\partial y_{k}}=-\frac{\partial v_{j}}{\partial x_{k}}
\end{array} \quad j, k=1, \ldots, n\right.
$$

These equations imply

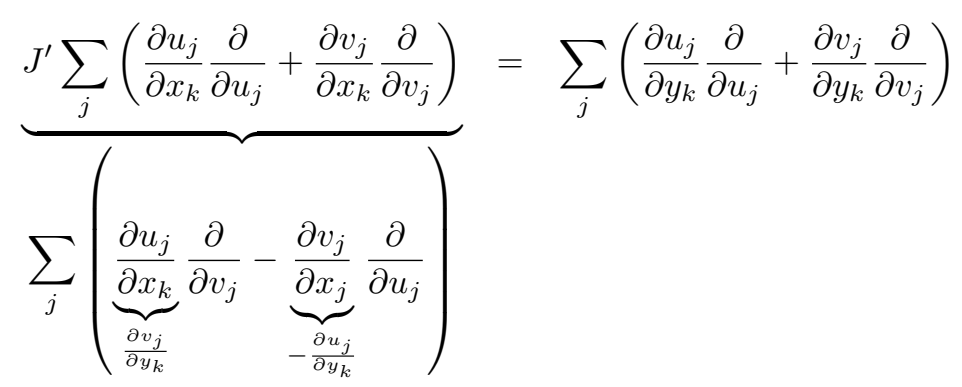

which matches the equation

$$
J \frac{\partial}{\partial x_{k}}=\frac{\partial}{\partial y_{k}}
$$




\subsection{Forms on Complex Manifolds}

Suppose that $M$ is a complex manifold and $J$ is its canonical almost complex structure. What does the splitting $\Omega^{k}(M ; \mathbb{C})=\oplus_{\ell+m=k} \Omega^{\ell, m}$ look like? ( $[22,48$, $66,109]$ are good references for this material.)

Let $\mathcal{U} \subseteq M$ be a coordinate neighborhood with complex coordinates $z_{1}, \ldots, z_{n}$, $z_{j}=x_{j}+i y_{j}$, and real coordinates $x_{1}, y_{1}, \ldots, x_{n}, y_{n}$. At $p \in \mathcal{U}$,

$$
\begin{aligned}
& T_{p} M=\mathbb{R}-\operatorname{span}\left\{\left.\frac{\partial}{\partial x_{j}}\right|_{p},\left.\frac{\partial}{\partial y_{j}}\right|_{p}\right\} \\
& T_{p} M \otimes \mathbb{C}=\mathbb{C}-\operatorname{span}\left\{\left.\frac{\partial}{\partial x_{j}}\right|_{p},\left.\frac{\partial}{\partial y_{j}}\right|_{p}\right\} \\
& =\underbrace{\mathbb{C}-\operatorname{span}\left\{\frac{1}{2}\left(\left.\frac{\partial}{\partial x_{j}}\right|_{p}-\left.i \frac{\partial}{\partial y_{j}}\right|_{p}\right)\right\}} \oplus \underbrace{\mathbb{C}-\operatorname{span}\left\{\frac{1}{2}\left(\left.\frac{\partial}{\partial x_{j}}\right|_{p}+\left.i \frac{\partial}{\partial y_{j}}\right|_{p}\right)\right\}}_{T_{1,0}} \\
& T_{1,0}=(+i) \text {-eigenspace of } J \quad T_{0,1}=(-i) \text {-eigenspace of } J \\
& J\left(\frac{\partial}{\partial x_{j}}-i \frac{\partial}{\partial y_{j}}\right)=i\left(\frac{\partial}{\partial x_{j}}-i \frac{\partial}{\partial y_{j}}\right) \quad J\left(\frac{\partial}{\partial x_{j}}+i \frac{\partial}{\partial y_{j}}\right)=-i\left(\frac{\partial}{\partial x_{j}}+i \frac{\partial}{\partial y_{j}}\right)
\end{aligned}
$$

This can be written more concisely using:

\section{Definition 15.3}

$$
\frac{\partial}{\partial z_{j}}:=\frac{1}{2}\left(\frac{\partial}{\partial x_{j}}-i \frac{\partial}{\partial y_{j}}\right) \quad \text { and } \quad \frac{\partial}{\partial \bar{z}_{j}}:=\frac{1}{2}\left(\frac{\partial}{\partial x_{j}}+i \frac{\partial}{\partial y_{j}}\right) \text {. }
$$

Hence,

$$
\left(T_{1,0}\right)_{p}=\mathbb{C} \text {-span }\left\{\left.\frac{\partial}{\partial z_{j}}\right|_{p}: j=1, \ldots, n\right\},\left(T_{0,1}\right)_{p}=\mathbb{C}-\operatorname{span}\left\{\left.\frac{\partial}{\partial \bar{z}_{j}}\right|_{p}: j=1, \ldots, n\right\} .
$$

Similarly,

$$
\begin{gathered}
T^{*} M \otimes \mathbb{C}=\mathbb{C}-\operatorname{span}\left\{d x_{j}, d y_{j}: j=1, \ldots, n\right\} \\
=\underbrace{\mathbb{C}-\operatorname{span}\left\{d x_{j}+i d y_{j}: j=1, \ldots, n\right\}}_{T^{1,0}} \oplus \underbrace{\mathbb{C}-\operatorname{span}\left\{d x_{j}-i d y_{j}: j=1, \ldots, n\right\}}_{T^{0,1}} \\
\left(d x_{j}+i d y_{j}\right) \circ J=i\left(d x_{j}+i d y_{j}\right)
\end{gathered} \quad \begin{gathered}
\left(d x_{j}-i d y_{j}\right) \circ J=-i\left(d x_{j}-i d y_{j}\right)
\end{gathered}
$$

Putting

$$
d z_{j}=d x_{j}+i d y_{j} \quad \text { and } \quad d \bar{z}_{j}=d x_{j}-i d y_{j}
$$

we obtain

$$
T^{1,0}=\mathbb{C}-\operatorname{span}\left\{d z_{j}: j=1, \ldots, n\right\}, \quad T^{0,1}=\mathbb{C}-\operatorname{span}\left\{d \bar{z}_{j}: j=1, \ldots, n\right\} .
$$


On the coordinate neighborhood $\mathcal{U}$,

$$
\begin{aligned}
& (1,0) \text {-forms }=\left\{\sum_{j} b_{j} d z_{j} \mid b_{j} \in C^{\infty}(\mathcal{U} ; \mathbb{C})\right\} \\
& (0,1) \text {-forms }=\left\{\sum_{j} b_{j} d \bar{z}_{j} \mid b_{j} \in C^{\infty}(\mathcal{U} ; \mathbb{C})\right\} \\
& (2,0) \text {-forms }=\left\{\sum_{j_{1}<j_{2}} b_{j_{1}, j_{2}} d z_{j_{1}} \wedge d z_{j_{2}} \mid b_{j_{1}, j_{2}} \in C^{\infty}(\mathcal{U} ; \mathbb{C})\right\} \\
& (1,1) \text {-forms }=\left\{\sum_{j_{1}, j_{2}} b_{j_{1}, j_{2}} d z_{j_{1}} \wedge d \bar{z}_{j_{2}} \mid b_{j_{1}, j_{2}} \in C^{\infty}(\mathcal{U} ; \mathbb{C})\right\} \\
& (0,2) \text {-forms }=\left\{\sum_{j_{1}<j_{2}} b_{j_{1}, j_{2}} d \bar{z}_{j_{1}} \wedge d \bar{z}_{j_{2}} \mid b_{j_{1}, j_{2}} \in C^{\infty}(\mathcal{U} ; \mathbb{C})\right\}
\end{aligned}
$$

If we use multi-index notation:

$$
\begin{array}{rlr}
J & =\left(j_{1}, \ldots, j_{m}\right) \quad 1 \leq j_{1}<\ldots<j_{m} \leq n \\
|J| & =m \\
d z_{J} & =d z_{j_{1}} \wedge d z_{j_{2}} \wedge \ldots \wedge d z_{j_{m}}
\end{array}
$$

then

$$
\Omega^{\ell, m}=(\ell, m) \text {-forms }=\left\{\sum_{|J|=\ell,|K|=m} b_{J, K} d z_{J} \wedge d \bar{z}_{K} \mid b_{J, K} \in C^{\infty}(\mathcal{U} ; \mathbb{C})\right\} .
$$

\subsection{Differentials}

On a coordinate neighborhood $\mathcal{U}$, a form $\beta \in \Omega^{k}(M ; \mathbb{C})$ may be written as

$$
\beta=\sum_{|J|+|K|=k} a_{J, K} d x_{J} \wedge d y_{K}, \quad \text { with } a_{J, K} \in C^{\infty}(\mathcal{U} ; \mathbb{C})
$$

We would like to know whether the following equality holds:

$$
d \beta=\sum\left(\partial a_{J, K}+\bar{\partial} a_{J, K}\right) d x_{J} \wedge d y_{K} \stackrel{?}{=}(\partial+\bar{\partial}) \sum a_{J, K} d x_{J} \wedge d y_{K}
$$

If we use the identities

$$
\left\{\begin{array} { l } 
{ d x _ { j } + i d y _ { j } = d z _ { j } } \\
{ d x _ { j } - i d y _ { j } = d \overline { z } _ { j } }
\end{array} \Longleftrightarrow \left\{\begin{array}{l}
d x_{j}=\frac{1}{2}\left(d z_{j}+d \bar{z}_{j}\right) \\
d y_{j}=\frac{1}{2 i}\left(d z_{j}-d \bar{z}_{j}\right)
\end{array}\right.\right.
$$


after substituting and reshuffling, we obtain

$$
\begin{aligned}
& \beta=\sum_{|J|+|K|=k} b_{J, K} d z_{J} \wedge d \bar{z}_{K} \\
& =\sum_{\ell+m=k} \underbrace{\left(\sum_{|J|=\ell,|K|=m} b_{J, K} d z_{J} \wedge d \bar{z}_{K}\right)}_{\in \Omega^{\ell, m}} \\
& d \beta=\sum_{\ell+m=k}\left(\sum_{|J|=\ell,|K|=m} d b_{J, K} \wedge d z_{J} \wedge d \bar{z}_{K}\right) \\
& =\sum_{\ell+m=k} \sum_{|J|=\ell,|K|=m}\left(\partial b_{J, K}+\bar{\partial} b_{J, K}\right) \wedge d z_{J} \wedge d \bar{z}_{K} \\
& \text { (because } d=\partial+\bar{\partial} \text { on functions) } \\
& =\sum_{\ell+m=k}(\underbrace{\sum_{|J|=\ell,|K|=m} \partial b_{J, K} \wedge d z_{J} \wedge d \bar{z}_{K}}_{\in \Omega^{\ell+1, m}}+\underbrace{\sum_{|J|=\ell,|K|=m} \bar{\partial}_{J, K} \wedge d z_{J} \wedge d \bar{z}_{K}}_{\in \Omega^{\ell, m+1}}) \\
& =\partial \beta+\bar{\partial} \beta .
\end{aligned}
$$

Therefore, $d=\partial+\bar{\partial}$ on forms of any degree for a complex manifold.

Conclusion. If $M$ is a complex manifold, then $d=\partial+\bar{\partial}$. (For an almost complex manifold this fails because there are no coordinate functions $z_{j}$ to give a suitable basis of 1 -forms.)

Remark. If $b \in C^{\infty}(\mathcal{U} ; \mathbb{C})$, in terms of $z$ and $\bar{z}$, we obtain the following formulas:

$$
\begin{aligned}
d b & =\sum_{j}\left(\frac{\partial b}{\partial x_{j}} d x_{j}+\frac{\partial b}{\partial y_{j}} d y_{j}\right) \\
& =\sum_{j}\left[\frac{1}{2}\left(\frac{\partial b}{\partial x_{j}}-i \frac{\partial b}{\partial y_{j}}\right)\left(d x_{j}+i d y_{j}\right)+\frac{1}{2}\left(\frac{\partial b}{\partial x_{j}}+i \frac{\partial b}{\partial y_{j}}\right)\left(d x_{j}-i d y_{j}\right)\right] \\
& =\sum_{j}\left(\frac{\partial b}{\partial z_{j}} d z_{j}+\frac{\partial b}{\partial \bar{z}_{j}} d \bar{z}_{j}\right) .
\end{aligned}
$$

Hence:

$$
\left\{\begin{array}{l}
\partial b=\pi^{1,0} d b=\sum_{j} \frac{\partial b}{\partial z_{j}} d z_{j} \\
\bar{\partial} b=\pi^{0,1} d b=\sum_{j} \frac{\partial b}{\partial \bar{z}_{j}} d \bar{z}_{j}
\end{array}\right.
$$


In the case where $\beta \in \Omega^{\ell, m}$, we have

$$
\begin{aligned}
d \beta & =\partial \beta+\bar{\partial} \beta=(\ell+1, m) \text {-form }+(\ell, m+1) \text {-form } \\
0=d^{2} \beta & =(\ell+2, m) \text {-form }+(\ell+1, m+1) \text {-form }+(\ell, m+2) \text {-form } \\
& =\underbrace{\partial^{2} \beta}_{0}+\underbrace{(\partial \bar{\partial}+\bar{\partial} \partial) \beta}_{0}+\underbrace{\bar{\partial}^{2} \beta}_{0} .
\end{aligned}
$$

Hence, $\bar{\partial}^{2}=0$.

The Dolbeault theorem states that for complex manifolds

$$
H_{\text {Dolbeault }}^{\ell, m}(M)=H^{m}\left(M ; \mathcal{O}\left(\Omega^{(\ell, 0)}\right)\right),
$$

where $\mathcal{O}\left(\Omega^{(\ell, 0)}\right)$ is the sheaf of forms of type $(\ell, 0)$ over $M$.

Theorem 15.4 (Newlander-Nirenberg, 1957 [89])

Let $(M, J)$ be an almost complex manifold. Let $\mathcal{N}$ be the Nijenhuis tensor (defined in Homework 10). Then:

$$
\begin{aligned}
M \text { is a complex manifold } & \Longleftrightarrow J \text { is integrable } \\
& \Longleftrightarrow \mathcal{N} \equiv 0 \\
& \Longleftrightarrow d=\partial+\bar{\partial} \\
& \Longleftrightarrow \bar{\partial}^{2}=0 \\
& \left.\Longleftrightarrow \pi^{2,0} d\right|_{\Omega^{0,1}}=0 .
\end{aligned}
$$

For the proof of this theorem, besides the original reference, see also [22, 30, $48,66,109]$. Naturally most almost complex manifolds have $d \neq \partial+\bar{\partial}$. 


\section{Homework 11: Complex Projective Space}

The complex projective space $\mathbb{C P}^{n}$ is the space of complex lines in $\mathbb{C}^{n+1}$ :

$\mathbb{C} \mathbb{P}^{n}$ is obtained from $\mathbb{C}^{n+1} \backslash\{0\}$ by making the identifications $\left(z_{0}, \ldots, z_{n}\right) \sim$ $\left(\lambda z_{0}, \ldots, \lambda z_{n}\right)$ for all $\lambda \in \mathbb{C} \backslash\{0\}$. One denotes by $\left[z_{0}, \ldots, z_{n}\right]$ the equivalence class of $\left(z_{0}, \ldots, z_{n}\right)$, and calls $z_{0}, \ldots, z_{n}$ the homogeneous coordinates of the point $p=\left[z_{0}, \ldots, z_{n}\right]$. (The homogeneous coordinates are, of course, only determined up to multiplication by a non-zero complex number $\lambda$.)

Let $\mathcal{U}_{i}$ be the subset of $\mathbb{C P}^{n}$ consisting of all points $p=\left[z_{0}, \ldots, z_{n}\right]$ for which $z_{i} \neq 0$. Let $\varphi_{i}: \mathcal{U}_{i} \rightarrow \mathbb{C}^{n}$ be the map

$$
\varphi_{i}\left(\left[z_{0}, \ldots, z_{n}\right]\right)=\left(\frac{z_{0}}{z_{i}}, \ldots, \frac{z_{i-1}}{z_{i}}, \frac{z_{i+1}}{z_{i}}, \ldots, \frac{z_{n}}{z_{i}}\right) .
$$

1. Show that the collection

$$
\left\{\left(\mathcal{U}_{i}, \mathbb{C}^{n}, \varphi_{i}\right), i=0, \ldots, n\right\}
$$

is an atlas in the complex sense, i.e., the transition maps are biholomorphic. Conclude that $\mathbb{C P}^{n}$ is a complex manifold.

Hint: Work out the transition maps associated with $\left(\mathcal{U}_{0}, \mathbb{C}^{n}, \varphi_{0}\right)$ and $\left(\mathcal{U}_{1}, \mathbb{C}^{n}, \varphi_{1}\right)$. Show that the transition diagram has the form

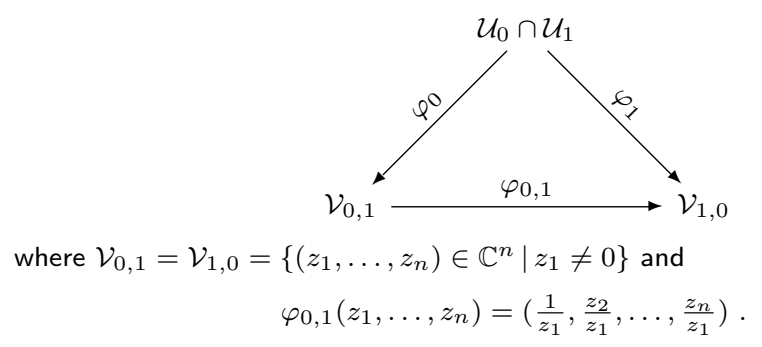

2. Show that the 1-dimensional complex manifold $\mathbb{C P} \mathbb{P}^{1}$ is diffeomorphic, as a real 2-dimensional manifold, to $S^{2}$.

Hint: Stereographic projection. 


\section{Kähler Forms}

\subsection{Kähler Forms}

Definition 16.1 A Kähler manifold is a symplectic manifold $(M, \omega)$ equipped with an integrable compatible almost complex structure. The symplectic form $\omega$ is then called a Kähler form.

It follows immediately from the previous definition that

$$
\begin{aligned}
(M, \omega) \text { is Kähler } & \Longrightarrow M \text { is a complex manifold } \\
& \Longrightarrow\left\{\begin{array}{l}
\Omega^{k}(M ; \mathbb{C})=\oplus_{\ell+m=k} \Omega^{\ell, m} \\
d=\partial+\bar{\partial}
\end{array}\right.
\end{aligned}
$$

where

$$
\begin{aligned}
& \partial=\pi^{\ell+1, m} \circ d: \Omega^{\ell, m} \rightarrow \Omega^{\ell+1, m} \\
& \bar{\partial}=\pi^{\ell, m+1} \circ d: \Omega^{\ell, m} \rightarrow \Omega^{\ell, m+1}
\end{aligned} .
$$

On a complex chart $\left(\mathcal{U}, z_{1}, \ldots, z_{n}\right), n=\operatorname{dim}_{\mathbb{C}} M$,

$$
\Omega^{\ell, m}=\left\{\sum_{|J|=\ell,|K|=m} b_{J K} d z_{J} \wedge d \bar{z}_{K} \mid b_{J K} \in C^{\infty}(\mathcal{U} ; \mathbb{C})\right\}
$$

where

$$
\begin{aligned}
& J=\left(j_{1}, \ldots, j_{\ell}\right), \quad j_{1}<\ldots<j_{\ell}, \quad d z_{J}=d z_{j_{1}} \wedge \ldots \wedge d z_{j_{\ell}}, \\
& K=\left(k_{1}, \ldots, k_{m}\right), \quad k_{1}<\ldots<k_{m}, \quad d \bar{z}_{K}=d \bar{z}_{k_{1}} \wedge \ldots \wedge d \bar{z}_{k_{m}} \text {. }
\end{aligned}
$$

On the other hand,

$$
(M, \omega) \text { is Kähler } \Longrightarrow \omega \text { is a symplectic form . }
$$

- Where does $\omega$ fit with respect to the above decomposition?

A Kähler form $\omega$ is

1. a 2-form,

2. compatible with the complex structure,

3. closed,

4. real-valued, and

5. nondegenerate.

These properties translate into: 
1. $\Omega^{2}(M ; \mathbb{C})=\Omega^{2,0} \oplus \Omega^{1,1} \oplus \Omega^{0,2}$.

On a local complex chart $\left(\mathcal{U}, z_{1}, \ldots, z_{n}\right)$,

$$
\omega=\sum a_{j k} d z_{j} \wedge d z_{k}+\sum b_{j k} d z_{j} \wedge d \bar{z}_{k}+\sum c_{j k} d \bar{z}_{j} \wedge d \bar{z}_{k}
$$

for some $a_{j k}, b_{j k}, c_{j k} \in C^{\infty}(\mathcal{U} ; \mathbb{C})$.

2. $J$ is a symplectomorphism, that is, $J^{*} \omega=\omega$ where $\left(J^{*} \omega\right)(u, v):=\omega(J u, J v)$.

$$
\begin{aligned}
J^{*} d z_{j} & =d z_{j} \circ J=i d z_{j} \\
J^{*} d \bar{z}_{j} & =d \bar{z}_{j} \circ J=-i d \bar{z}_{j} \\
J^{*} \omega & =\sum(i \cdot i) a_{j k} d z_{j} \wedge d z_{k}+i(\stackrel{\|}{\|}-i) \sum b_{j k} d z_{j} \wedge d \bar{z}_{k}+(-i)^{2} \sum c_{j k} d \bar{z}_{j} \wedge d \bar{z}_{k} \\
J^{*} \omega & =\omega \quad \Longleftrightarrow \quad a_{j k}=0=c_{j k}, \text { all } j, k \quad \Longleftrightarrow \omega \in \Omega^{1,1} .
\end{aligned}
$$

3. $0=d \omega=\underbrace{\partial \omega}_{(2,1)-\text { form }}+\underbrace{\bar{\partial} \omega}_{(1,2)-\text { form }} \Longrightarrow \begin{cases}\partial \omega=0 & \omega \text { is } \partial \text {-closed } \\ \bar{\partial} \omega=0 & \omega \text { is } \bar{\partial} \text {-closed }\end{cases}$

Hence, $\omega$ defines a Dolbeault $(1,1)$ cohomology class,

$$
[\omega] \in H_{\text {Dolbeault }}^{1,1}(M) .
$$

Putting $b_{j k}=\frac{i}{2} h_{j k}$,

$$
\omega=\frac{i}{2} \sum_{j, k=1}^{n} h_{j k} d z_{j} \wedge d \bar{z}_{k}, \quad h_{j k} \in C^{\infty}(\mathcal{U} ; \mathbb{C}) .
$$

4. $\omega$ real-valued $\Longleftrightarrow \omega=\bar{\omega}$.

$$
\begin{gathered}
\bar{\omega}=-\frac{i}{2} \sum \overline{h_{j k}} d \bar{z}_{j} \wedge d z_{k}=\frac{i}{2} \sum \overline{h_{j k}} d z_{k} \wedge d \bar{z}_{j}=\frac{i}{2} \sum \overline{h_{k j}} d z_{j} \wedge d \bar{z}_{k} \\
\omega \text { real } \Longleftrightarrow h_{j k}=\overline{h_{k j}},
\end{gathered}
$$

i.e., at every point $p \in \mathcal{U}$, the $n \times n$ matrix $\left(h_{j k}(p)\right)$ is hermitian.

5. nondegeneracy: $\omega^{n}=\underbrace{\omega \wedge \ldots \wedge \omega}_{n} \neq 0$.

Exercise. Check that

$$
\omega^{n}=n !\left(\frac{i}{2}\right)^{n} \operatorname{det}\left(h_{j k}\right) d z_{1} \wedge d \bar{z}_{1} \wedge \ldots \wedge d z_{n} \wedge d \bar{z}_{n} .
$$

Now

$$
\omega \text { nondegenerate } \Longleftrightarrow \operatorname{det}_{\mathbb{C}}\left(h_{j k}\right) \neq 0,
$$

i.e., at every $p \in M,\left(h_{j k}(p)\right)$ is a nonsingular matrix. 
2. Again the positivity condition: $\omega(v, J v)>0, \forall v \neq 0$.

Exercise. Show that $\left(h_{j k}(p)\right)$ is positive-definite.

$$
\omega \text { positive } \Longleftrightarrow\left(h_{j k}\right) \gg 0,
$$

i.e., at each $p \in \mathcal{U},\left(h_{j k}(p)\right)$ is positive-definite.

Conclusion. Kähler forms are $\partial$ - and $\bar{\partial}$-closed $(1,1)$-forms, which are given on a local chart $\left(\mathcal{U}, z_{1}, \ldots, z_{n}\right)$ by

$$
\omega=\frac{i}{2} \sum_{j, k=1}^{n} h_{j k} d z_{j} \wedge d \bar{z}_{k}
$$

where, at every point $p \in \mathcal{U},\left(h_{j k}(p)\right)$ is a positive-definite hermitian matrix.

\subsection{An Application}

Theorem 16.2 (Banyaga) Let $M$ be a compact complex manifold. Let $\omega_{0}$ and $\omega_{1}$ be Kähler forms on $M$. If $\left[\omega_{0}\right]=\left[\omega_{1}\right] \in H_{\mathrm{deRham}}^{2}(M)$, then $\left(M, \omega_{0}\right)$ and $\left(M, \omega_{1}\right)$ are symplectomorphic.

Proof. Any combination $\omega_{t}=(1-t) \omega_{0}+t \omega_{1}$ is symplectic for $0 \leq t \leq 1$, because, on a complex chart $\left(\mathcal{U}, z_{1}, \ldots, z_{n}\right)$, where $n=\operatorname{dim}_{\mathbb{C}} M$, we have

$$
\begin{aligned}
\omega_{0}= & \frac{i}{2} \sum h_{j k}^{0} d z_{j} \wedge d \bar{z}_{k} \\
\omega_{1}= & \frac{i}{2} \sum h_{j k}^{1} d z_{j} \wedge d \bar{z}_{k} \\
\omega_{t}= & \frac{i}{2} \sum h_{j k}^{t} d z_{j} \wedge d \bar{z}_{k}, \quad \text { where } h_{j k}^{t}=(1-t) h_{j k}^{0}+t h_{j k}^{1} . \\
& \left(h_{j k}^{0}\right) \gg 0,\left(h_{j k}^{1}\right) \gg 0 \Longrightarrow\left(h_{j k}^{t}\right) \gg 0 .
\end{aligned}
$$

Apply the Moser theorem (Theorem 7.2).

\subsection{Recipe to Obtain Kähler Forms}

Definition 16.3 Let $M$ be a complex manifold. A function $\rho \in C^{\infty}(M ; \mathbb{R})$ is strictly plurisubharmonic (s.p.s.h.) if, on each local complex chart $\left(\mathcal{U}, z_{1}, \ldots\right.$, $\left.z_{n}\right)$, where $n=\operatorname{dim}_{\mathbb{C}} M$, the matrix $\left(\frac{\partial^{2} \rho}{\partial z_{j} \partial \bar{z}_{k}}(p)\right)$ is positive-definite at all $p \in \mathcal{U}$.

Proposition 16.4 Let $M$ be a complex manifold and let $\rho \in C^{\infty}(M ; \mathbb{R})$ be s.p.s.h.. Then

$$
\omega=\frac{i}{2} \partial \bar{\partial} \rho \quad \text { is Kähler . }
$$


A function $\rho$ as in the previous proposition is called a (global) Kähler potential.

Proof. Simply observe that:

$$
\begin{gathered}
\left\{\begin{array}{l}
\partial \omega=\frac{i}{2} \overbrace{\partial^{2}}^{0} \bar{\partial} \rho=0 \\
\bar{\partial} \omega=\frac{i}{2} \underbrace{\bar{\partial} \partial}_{-\partial \bar{\partial} \partial} \rho=-\frac{i}{2} \partial \underbrace{\bar{\partial}^{2}}_{0} \rho=0
\end{array}\right. \\
d \omega=\partial \omega+\bar{\partial} \omega=0 \Longrightarrow \omega \text { is closed } . \\
\bar{\omega}=-\frac{i}{2} \bar{\partial} \partial \rho=\frac{i}{2} \partial \bar{\partial} \rho=\omega \Longrightarrow \omega \text { is real } . \\
\omega \in \Omega^{1,1} \Longrightarrow J^{*} \omega=\omega \Longrightarrow \omega(\cdot, J \cdot) \text { is symmetric } .
\end{gathered}
$$

Exercise. Show that, for $f \in C^{\infty}(\mathcal{U} ; \mathbb{C})$,

$$
\partial f=\sum \frac{\partial f}{\partial z_{j}} d z_{j} \quad \text { and } \quad \bar{\partial} f=\sum \frac{\partial f}{\partial \bar{z}_{j}} d \bar{z}_{j} .
$$

Since the right-hand sides are in $\Omega^{1,0}$ and $\Omega^{0,1}$, respectively, it suffices to show that the sum of the two expressions is $d f$.

$$
\begin{gathered}
\omega=\frac{i}{2} \partial \bar{\partial} \rho=\frac{i}{2} \sum \frac{\partial}{\partial z_{j}}\left(\frac{\partial \rho}{\partial \bar{z}_{k}}\right) d z_{j} \wedge d \bar{z}_{k}=\frac{i}{2} \sum \underbrace{\left(\frac{\partial^{2} \rho}{\partial z_{j} \partial \bar{z}_{k}}\right)}_{h_{j k}} d z_{j} \wedge d \bar{z}_{k} . \\
\rho \text { is s.p.s.h } \Longrightarrow\left(h_{j k}\right) \gg 0 \Longrightarrow \omega(\cdot, J \cdot) \text { is positive } .
\end{gathered}
$$

In particular, $\omega$ is nondegenerate.

Example. Let $M=\mathbb{C}^{n} \simeq \mathbb{R}^{2 n}$, with complex coordinates $\left(z_{1}, \ldots, z_{n}\right)$ and corresponding real coordinates $\left(x_{1}, y_{1}, \ldots, x_{n}, y_{n}\right)$ via $z_{j}=x_{j}+i y_{j}$. Let

$$
\rho\left(x_{1}, y_{1}, \ldots, x_{n}, y_{n}\right)=\sum_{j=1}^{n}\left(x_{j}^{2}+y_{j}^{2}\right)=\sum\left|z_{j}\right|^{2}=\sum z_{j} \bar{z}_{j} .
$$

Then

$$
\frac{\partial}{\partial z_{j}} \frac{\partial \rho}{\partial \bar{z}_{k}}=\frac{\partial}{\partial z_{j}} z_{k}=\delta_{j k}
$$

so

$$
\left(h_{j k}\right)=\left(\frac{\partial^{2} \rho}{\partial z_{j} \partial \bar{z}_{k}}\right)=\left(\delta_{j k}\right)=\operatorname{Id} \gg 0 \Longrightarrow \rho \text { is s.p.s.h. . }
$$


The corresponding Kähler form

$$
\begin{aligned}
\omega & =\frac{i}{2} \partial \bar{\partial} \rho=\frac{i}{2} \sum_{j, k} \delta_{j k} d z_{j} \wedge d \bar{z}_{k} \\
& =\frac{i}{2} \sum_{j} d z_{j} \wedge d \bar{z}_{j}=\sum_{j} d x_{j} \wedge d y_{j} \quad \text { is the standard form } .
\end{aligned}
$$

\subsection{Local Canonical Form for Kähler Forms}

There is a local converse to the previous construction of Kähler forms.

Theorem 16.5 Let $\omega$ be a closed real-valued $(1,1)$-form on a complex manifold $M$ and let $p \in M$. Then there exist a neighborhood $\mathcal{U}$ of $p$ and $\rho \in C^{\infty}(\mathcal{U} ; \mathbb{R})$ such that, on $\mathcal{U}$,

$$
\omega=\frac{i}{2} \partial \bar{\partial} \rho .
$$

The function $\rho$ is then called a (local) Kähler potential.

The proof requires holomorphic versions of Poincaré's lemma, namely, the local triviality of Dolbeault groups:

$$
\forall p \in M \quad \exists \text { neighborhood } \mathcal{U} \text { of } p \text { such that } \quad H_{\text {Dolbeault }}^{\ell, m}(\mathcal{U})=0, m>0,
$$

and the local triviality of the holomorphic de Rham groups; see [48].

Proposition 16.6 Let $M$ be a complex manifold, $\rho \in C^{\infty}(M ; \mathbb{R})$ s.p.s.h., $X$ a complex submanifold, and $i: X \hookrightarrow M$ the inclusion map. Then $i^{*} \rho$ is s.p.s.h..

Proof. Let $\operatorname{dim}_{\mathbb{C}} M=n$ and $\operatorname{dim}_{\mathbb{C}} X=n-m$. For $p \in X$, choose a chart $\left(\mathcal{U}, z_{1}, \ldots, z_{n}\right)$ for $M$ centered at $p$ and adapted to $X$, i.e., $X \cap \mathcal{U}$ is given by $z_{1}=\ldots=z_{m}=0$. In this chart, $i^{*} \rho=\rho\left(0,0, \ldots, 0, z_{m+1}, \ldots, z_{n}\right)$.

$i^{*} p$ is s.p.s.h. $\Longleftrightarrow\left(\frac{\partial^{2} \rho}{\partial z_{m+j} \partial \bar{z}_{m+k}}\left(0, \ldots, 0, z_{m+1}, \ldots, z_{n}\right)\right)$ is positive-definite, which holds since this is a minor of $\left(\frac{\partial^{2}}{\partial z_{j} \partial \bar{z}_{k}}\left(0, \ldots, 0, z_{m+1}, \ldots, z_{n}\right)\right)$.

Corollary 16.7 Any complex submanifold of a Kähler manifold is also Kähler.

Definition 16.8 Let $(M, \omega)$ be a Kähler manifold, $X$ a complex submanifold, and $i: X \hookrightarrow M$ the inclusion. Then $\left(X, i^{*} \omega\right)$ is called a Kähler submanifold. 
Example. Complex vector space $\left(\mathbb{C}^{n}, \omega_{0}\right)$ where $\omega_{0}=\frac{i}{2} \sum d z_{j} \wedge d \bar{z}_{j}$ is Kähler. Every complex submanifold of $\mathbb{C}^{n}$ is Kähler.

Example. The complex projective space is

$$
\mathbb{C P}^{n}=\mathbb{C}^{n+1} \backslash\{0\} / \sim
$$

where

$$
\left(z_{0}, \ldots, z_{n}\right) \sim\left(\lambda z_{0}, \ldots, \lambda z_{n}\right), \quad \lambda \in \mathbb{C} \backslash\{0\} .
$$

The Fubini-Study form (see Homework 12) is Kähler. Therefore, every non-singular projective variety is a Kähler submanifold. Here we mean

$$
\begin{array}{rll}
\text { non-singular }= & \text { smooth } \\
\text { projective variety }= & \text { zero locus of a collection } \\
& \text { of homogeneous polynomials } .
\end{array}
$$




\section{Homework 12: The Fubini-Study Structure}

The purpose of the following exercises is to describe the natural Kähler structure on complex projective space, $\mathbb{C P}^{n}$.

1. Show that the function on $\mathbb{C}^{n}$

$$
z \longmapsto \log \left(|z|^{2}+1\right)
$$

is strictly plurisubharmonic. Conclude that the 2 -form

$$
\omega_{\mathrm{FS}}=\frac{i}{2} \partial \bar{\partial} \log \left(|z|^{2}+1\right)
$$

is a Kähler form. (It is usually called the Fubini-Study form on $\mathbb{C}^{n}$.)

Hint: A hermitian $n \times n$ matrix $H$ is positive definite if and only if $v^{*} H v>0$ for any $v \in \mathbb{C}^{n} \backslash\{0\}$, where $v^{*}$ is the transpose of the vector $\bar{v}$. To prove positivedefiniteness, either apply the Cauchy-Schwarz inequality, or use the following symmetry observation: $\mathrm{U}(n)$ acts transitively on $S^{2 n-1}$ and $\omega_{\mathrm{FS}}$ is $\mathrm{U}(n)$-invariant, thus it suffices to show positive-definiteness along one direction.

2. Let $\mathcal{U}$ be the open subset of $\mathbb{C}^{n}$ defined by the inequality $z_{1} \neq 0$, and let $\varphi: \mathcal{U} \rightarrow \mathcal{U}$ be the map

$$
\varphi\left(z_{1}, \ldots, z_{n}\right)=\frac{1}{z_{1}}\left(1, z_{2}, \ldots, z_{n}\right) .
$$

Show that $\varphi$ maps $\mathcal{U}$ biholomorphically onto $\mathcal{U}$ and that

$$
\varphi^{*} \log \left(|z|^{2}+1\right)=\log \left(|z|^{2}+1\right)+\log \frac{1}{\left|z_{1}\right|^{2}} .
$$

3. Notice that, for every point $p \in \mathcal{U}$, we can write the second term in $(\star)$ as the sum of a holomorphic and an anti-holomorphic function:

$$
-\log z_{1}-\log \overline{z_{1}}
$$

on a neighborhood of $p$. Conclude that

$$
\partial \bar{\partial} \varphi^{*} \log \left(|z|^{2}+1\right)=\partial \bar{\partial} \log \left(|z|^{2}+1\right)
$$

and hence that $\varphi^{*} \omega_{\mathrm{FS}}=\omega_{\mathrm{FS}}$.

Hint: You need to use the fact that the pullback by a holomorphic map $\varphi^{*}$ commutes with the $\partial$ and $\bar{\partial}$ operators. This is a consequence of $\varphi^{*}$ preserving form type, $\varphi^{*}\left(\Omega^{p, q}\right) \subseteq \Omega^{p, q}$, which in turn is implied by $\varphi^{*} d z_{j}=\partial \varphi_{j} \subseteq \Omega^{1,0}$ and $\varphi^{*} d \overline{z_{j}}=\bar{\partial} \overline{\varphi_{j}} \subseteq \bar{\Omega}^{0,1}$, where $\varphi_{j}$ is the $j$ th component of $\varphi$ with respect to local complex coordinates $\left(z_{1}, \ldots, z_{n}\right)$.

4. Recall that $\mathbb{C P}^{n}$ is obtained from $\mathbb{C}^{n+1} \backslash\{0\}$ by making the identifications $\left(z_{0}, \ldots, z_{n}\right) \sim\left(\lambda z_{0}, \ldots, \lambda z_{n}\right)$ for all $\lambda \in \mathbb{C} \backslash\{0\} ;\left[z_{0}, \ldots, z_{n}\right]$ is the equivalence class of $\left(z_{0}, \ldots, z_{n}\right)$. 
For $i=0,1, \ldots, n$, let

$$
\begin{aligned}
& \mathcal{U}_{i}=\left\{\left[z_{0}, \ldots, z_{n}\right] \in \mathbb{C P}^{n} \mid z_{i} \neq 0\right\} \\
& \varphi_{i}: \mathcal{U}_{i} \rightarrow \mathbb{C}^{n} \quad \varphi_{i}\left(\left[z_{0}, \ldots, z_{n}\right]\right)=\left(\frac{z_{0}}{z_{i}}, \ldots, \frac{z_{i-1}}{z_{i}}, \frac{z_{i+1}}{z_{i}}, \ldots, \frac{z_{n}}{z_{i}}\right) .
\end{aligned}
$$

Homework 11 showed that the collection $\left\{\left(\mathcal{U}_{i}, \mathbb{C}^{n}, \varphi_{i}\right), i=0, \ldots, n\right\}$ is a complex atlas (i.e., the transition maps are biholomorphic). In particular, it was shown that the transition diagram associated with $\left(\mathcal{U}_{0}, \mathbb{C}^{n}, \varphi_{0}\right)$ and $\left(\mathcal{U}_{1}, \mathbb{C}^{n}, \varphi_{1}\right)$ has the form

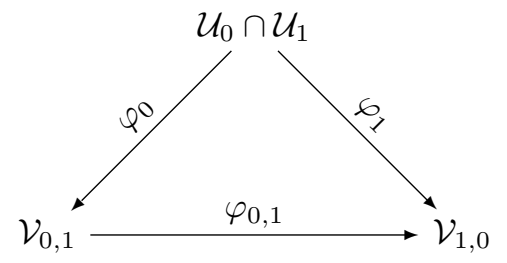

where $\mathcal{V}_{0,1}=\mathcal{V}_{1,0}=\left\{\left(z_{1}, \ldots, z_{n}\right) \in \mathbb{C}^{n} \mid z_{1} \neq 0\right\}$ and $\varphi_{0,1}\left(z_{1}, \ldots, z_{n}\right)=$ $\left(\frac{1}{z_{1}}, \frac{z_{2}}{z_{1}}, \ldots, \frac{z_{n}}{z_{1}}\right)$. Now the set $\mathcal{U}$ in exercise 2 is equal to the sets $\mathcal{V}_{0,1}$ and $\mathcal{V}_{1,0}$, and the map $\varphi$ coincides with $\varphi_{0,1}$.

Show that $\varphi_{0}^{*} \omega_{\mathrm{FS}}$ and $\varphi_{1}^{*} \omega_{\mathrm{FS}}$ are identical on the overlap $\mathcal{U}_{0} \cap \mathcal{U}_{1}$.

More generally, show that the Kähler forms $\varphi_{i}^{*} \omega_{\mathrm{FS}}$ "glue together" to define a Kähler structure on $\mathbb{C P}^{n}$. This is called the Fubini-Study form on complex projective space.

5. Prove that for $\mathbb{C P}^{1}$ the Fubini-Study form on the chart $\mathcal{U}_{0}=\left\{\left[z_{0}, z_{1}\right] \in\right.$ $\left.\mathbb{C P}^{1} \mid z_{0} \neq 0\right\}$ is given by the formula

$$
\omega_{\mathrm{FS}}=\frac{d x \wedge d y}{\left(x^{2}+y^{2}+1\right)^{2}}
$$

where $\frac{z_{1}}{z_{0}}=z=x+i y$ is the usual coordinate on $\mathbb{C}$.

6. Compute the total area of $\mathbb{C P} \mathbb{P}^{1}=\mathbb{C} \cup\{\infty\}$ with respect to $\omega_{\mathrm{FS}}$ :

$$
\int_{\mathbb{C P}^{1}} \omega_{\mathrm{FS}}=\int_{\mathbb{R}^{2}} \frac{d x \wedge d y}{\left(x^{2}+y^{2}+1\right)^{2}}
$$

7. Recall that $\mathbb{C P}^{1} \simeq S^{2}$ as real 2-dimensional manifolds (Homework 11). On $S^{2}$ there is the standard area form $\omega_{\text {std }}$ induced by regarding it as the unit sphere in $\mathbb{R}^{3}$ (Homework 6 ): in cylindrical polar coordinates $(\theta, h)$ on $S^{2}$ away from its poles $(0 \leq \theta<2 \pi$ and $-1 \leq h \leq 1)$, we have

$$
\omega_{\text {std }}=d \theta \wedge d h \text {. }
$$

Using stereographic projection, show that

$$
\omega_{\mathrm{FS}}=\frac{1}{4} \omega_{\mathrm{std}} .
$$




\section{Compact Kähler Manifolds}

\subsection{Hodge Theory}

Let $M$ be a complex manifold. A Kähler form $\omega$ on $M$ is a symplectic form which is compatible with the complex structure. Equivalently, a Kähler form $\omega$ is a $\partial$ and $\bar{\partial}$-closed form of type $(1,1)$ which, on a local chart $\left(\mathcal{U}, z_{1}, \ldots, z_{n}\right)$ is given by $\omega=\frac{i}{2} \sum_{j, k=1}^{n} h_{j k} d z_{j} \wedge d \bar{z}_{k}$, where, at each $x \in \mathcal{U},\left(h_{j k}(x)\right)$ is a positive-definite hermitian matrix. The pair $(M, \omega)$ is then called a Kähler manifold.

Theorem 17.1 (Hodge) On a compact Kähler manifold $(M, \omega)$ the Dolbeault cohomology groups satisfy

$$
H_{\mathrm{deRham}}^{k}(M ; \mathbb{C}) \simeq \bigoplus_{\ell+m=k} H_{\text {Dolbeault }}^{\ell, m}(M) \quad \text { (Hodge decomposition) }
$$

with $H^{\ell, m} \simeq \overline{H^{m, \ell}}$. In particular, the spaces $H_{\text {Dolbeault }}^{\ell, m}$ are finite-dimensional.

Hodge identified the spaces of cohomology classes of forms with spaces of actual forms, by picking the representative from each class which solves a certain differential equation, namely the harmonic representative.

(1) The Hodge $*$-operator.

Each tangent space $V=T_{x} M$ has a positive inner product $\langle\cdot, \cdot\rangle$, part of the riemannian metric in a compatible triple; we forget about the complex and symplectic structures until part (4).

Let $e_{1}, \ldots, e_{n}$ be a positively oriented orthonormal basis of $V$.

The star operator is a linear operator $*: \Lambda(V) \rightarrow \Lambda(V)$ defined by

$$
\begin{aligned}
*(1) & =e_{1} \wedge \ldots \wedge e_{n} \\
*\left(e_{1} \wedge \ldots \wedge e_{n}\right) & =1 \\
*\left(e_{1} \wedge \ldots \wedge e_{k}\right) & =e_{k+1} \wedge \ldots \wedge e_{n} .
\end{aligned}
$$

We see that $*: \Lambda^{k}(V) \rightarrow \Lambda^{n-k}(V)$ and satisfies $* *=(-1)^{k(n-k)}$.

(2) The codifferential and the laplacian are the operators defined by:

$$
\begin{aligned}
\delta=(-1)^{n(k+1)+1} * d * & : & \Omega^{k}(M) \rightarrow \Omega^{k-1}(M) \\
\Delta=d \delta+\delta d & : & \Omega^{k}(M) \rightarrow \Omega^{k}(M) .
\end{aligned}
$$

The operator $\Delta$ is also called the Laplace-Beltrami operator.

Exercise. Check that, on $\Omega^{0}\left(\mathbb{R}^{n}\right)=C^{\infty}\left(\mathbb{R}^{n}\right), \Delta=-\sum_{i=1}^{n} \frac{\partial^{2}}{\partial x_{i}^{2}}$.

Exercise. Check that $\Delta *=* \Delta$.

Suppose that $M$ is compact. Define an inner product on forms by

$$
\langle\cdot, \cdot\rangle: \Omega^{k} \times \Omega^{k} \rightarrow \mathbb{R}, \quad\langle\alpha, \beta\rangle=\int_{M} \alpha \wedge * \beta
$$


Exercise. Check that this is symmetric, positive-definite and satisfies $\langle d \alpha, \beta\rangle=$ $\langle\alpha, \delta \beta\rangle$.

Therefore, $\delta$ is often denoted by $d^{*}$ and called the adjoint of $d$. (When $M$ is not compact, we still have a formal adjoint of $d$ with respect to the nondegenerate bilinear pairing $\langle\cdot, \cdot\rangle: \Omega^{k} \times \Omega_{c}^{k} \rightarrow \mathbb{R}$ defined by a similar formula, where $\Omega_{c}^{k}$ is the space of compactly supported $k$-forms.) Also, $\Delta$ is self-adjoint:

Exercise. Check that $\langle\Delta \alpha, \beta\rangle=\langle\alpha, \Delta \beta\rangle$, and that $\langle\Delta \alpha, \alpha\rangle=|d \alpha|^{2}+$ $|\delta \alpha|^{2} \geq 0$, where $|\cdot|$ is the norm with respect to this inner product.

(3) The harmonic k-forms are the elements of $\mathcal{H}^{k}:=\left\{\alpha \in \Omega^{k} \mid \Delta \alpha=0\right\}$.

Note that $\Delta \alpha=0 \Longleftrightarrow d \alpha=\delta \alpha=0$. Since a harmonic form is $d$-closed, it defines a de Rham cohomology class.

Theorem 17.2 (Hodge) Every de Rham cohomology class on a compact oriented riemannian manifold $(M, g)$ possesses a unique harmonic representative, i.e.,

$$
\mathcal{H}^{k} \simeq H_{\text {deRham }}^{k}(M ; \mathbb{R})
$$

In particular, the spaces $\mathcal{H}^{k}$ are finite-dimensional. We also have the following orthogonal decomposition with respect to $\langle\cdot, \cdot\rangle$ :

$$
\begin{aligned}
\Omega^{k} & \simeq \mathcal{H}^{k} \oplus \Delta\left(\Omega^{k}(M)\right) \\
& \simeq \mathcal{H}^{k} \oplus d \Omega^{k-1} \oplus \delta \Omega^{k+1} \quad \text { (Hodge decomposition on forms) } .
\end{aligned}
$$

The proof involves functional analysis, elliptic differential operators, pseudodifferential operators and Fourier analysis; see [48, 109].

So far, this was ordinary Hodge theory, considering only the metric and not the complex structure.

\section{(4) Complex Hodge Theory.}

When $M$ is Kähler, the laplacian satisfies $\Delta=2\left(\bar{\partial} \bar{\partial}^{*}+\bar{\partial}^{*} \bar{\partial}\right.$ ) (see, for example, [48]) and preserves the decomposition according to type, $\Delta: \Omega^{\ell, m} \rightarrow$ $\Omega^{\ell, m}$. Hence, harmonic forms are also bigraded

$$
\mathcal{H}^{k}=\bigoplus_{\ell+m=k} \mathcal{H}^{\ell, m}
$$

Theorem 17.3 (Hodge) Every Dolbeault cohomology class on a compact Kähler manifold $(M, \omega)$ possesses a unique harmonic representative, i.e.,

$$
\mathcal{H}^{\ell, m} \simeq H_{\text {Dolbeault }}^{\ell, m}(M)
$$


and the spaces $\mathcal{H}^{\ell, m}$ are finite-dimensional. Hence, we have the following isomorphisms:

$$
H_{\text {deRham }}^{k}(M) \simeq \mathcal{H}^{k} \simeq \bigoplus_{\ell+m=k} \mathcal{H}^{\ell, m} \simeq \bigoplus_{\ell+m=k} H_{\text {Dolbeault }}^{\ell, m}(M)
$$

For the proof, see for instance $[48,109]$.

\subsection{Immediate Topological Consequences}

Let $b^{k}(M):=\operatorname{dim} H_{\mathrm{deRham}}^{k}(M)$ be the usual Betti numbers of $M$, and let $h^{\ell, m}(M):=\operatorname{dim} H_{\text {Dolbeault }}^{\ell, m}(M)$ be the so-called Hodge numbers of $M$.

$$
\text { Hodge Theorem } \Longrightarrow\left\{\begin{aligned}
b^{k} & =\sum_{\ell+m=k} h^{\ell, m} \\
h^{\ell, m} & =h^{m, \ell}
\end{aligned}\right.
$$

Some immediate topological consequences are:

1. On compact Kähler manifolds "the odd Betti numbers are even:"

$$
b^{2 k+1}=\sum_{\ell+m=2 k+1} h^{\ell, m}=2 \sum_{\ell=0}^{k} h^{\ell,(2 k+1-\ell)} \quad \text { is even . }
$$

2. On compact Kähler manifolds, $h^{1,0}=\frac{1}{2} b^{1}$ is a topological invariant.

3. On compact symplectic manifolds, "even Betti numbers are positive," because $\omega^{k}$ is closed but not exact $(k=0,1, \ldots, n)$.

Proof. If $\omega^{k}=d \alpha$, by Stokes' theorem, $\int_{M} \omega^{n}=\int_{M} d\left(\alpha \wedge \omega^{n-k}\right)=0$. This cannot happen since $\omega^{n}$ is a volume form.

4. On compact Kähler manifolds, the $h^{\ell, \ell}$ are positive.

Claim. $0 \neq\left[\omega^{\ell}\right] \in H_{\text {Dolbeault }}^{\ell, \ell}(M)$.

Proof.

$$
\begin{aligned}
\omega \in \Omega^{1,1} & \Longrightarrow \omega^{\ell} \in \Omega^{\ell, \ell} \\
d \omega=0 & \Longrightarrow 0=d \omega^{\ell}=\underbrace{\partial \omega^{\ell}}_{(\ell+1, \ell)}+\underbrace{\bar{\partial} \omega^{\ell}}_{(\ell, \ell+1)} \\
& \Longrightarrow \bar{\partial} \omega^{\ell}=0,
\end{aligned}
$$

so $\left[\omega^{\ell}\right]$ defines an element of $H_{\text {Dolbeault }}^{\ell, \ell}$. Why is $\omega^{\ell}$ not $\bar{\partial}$-exact?

If $\omega^{\ell}=\bar{\partial} \beta$ for some $\beta \in \Omega^{\ell-1, \ell}$, then

$$
\omega^{n}=\omega^{\ell} \wedge \omega^{n-\ell}=\bar{\partial}\left(\beta \wedge \omega^{n-\ell}\right) \Longrightarrow 0=\left[\omega^{n}\right] \in H_{\text {Dolbeault }}^{n, n}(M) .
$$

But $\left[\omega^{n}\right] \neq 0$ in $H_{\text {deRham }}^{2 n}(M ; \mathbb{C}) \simeq H_{\text {Dolbeault }}^{n, n}(M)$ since it is a volume form. 
There are other constraints on the Hodge numbers of compact Kähler manifolds, and ongoing research on how to compute $H_{\text {Dolbeault }}^{\ell, m}$. A popular picture to describe the relations is the Hodge diamond:

$$
\begin{array}{ccccc} 
& & h^{n, n} & & \\
& h^{n, n-1} & & h^{n-1, n} & \\
& & h^{n-1, n-1} & & h^{n-2, n} \\
& & \vdots & & \\
h^{n, n-2} & & h^{1,1} & & h^{0,2} \\
& h^{1,0} & & h^{0,1} & \\
& & h^{0,0} & &
\end{array}
$$

Complex conjugation gives symmetry with respect to the middle vertical, whereas the Hodge $*$ induces symmetry about the center of the diamond. The middle vertical axis is all non-zero. There are further symmetries induced by isomorphisms given by wedging with $\omega$.

The Hodge conjecture relates $H_{\text {Dolbeault }}^{\ell, \ell}(M) \cap H^{2 \ell}(M ; \mathbb{Z})$ for projective manifolds $M$ (i.e., submanifolds of complex projective space) to $\operatorname{codim}_{\mathbb{C}}=\ell$ complex submanifolds of $M$.

\subsection{Compact Examples and Counterexamples}
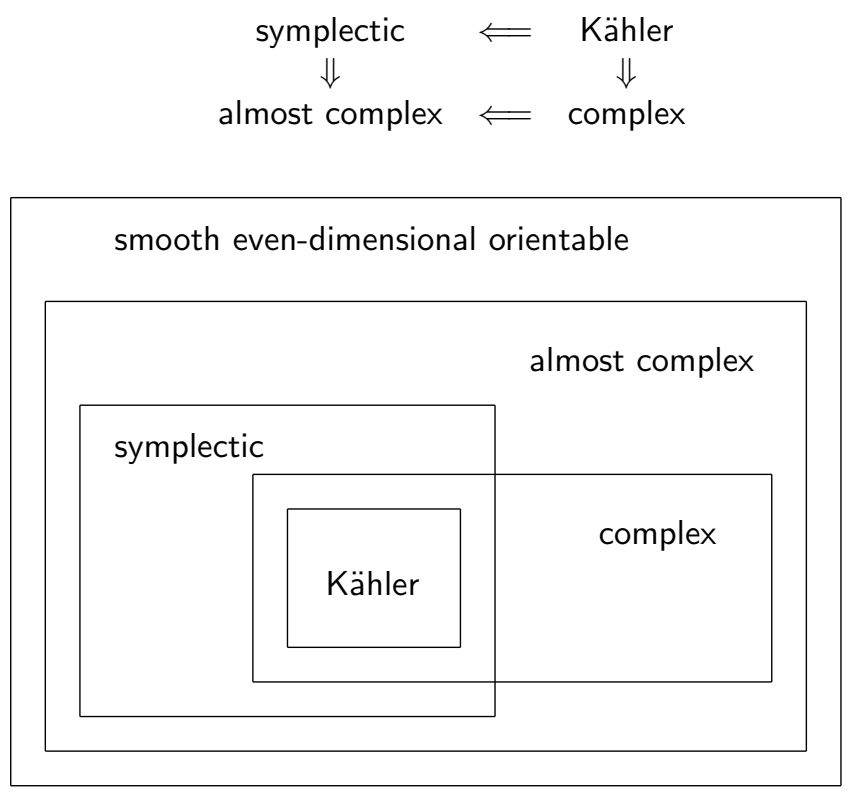

Is each of these regions nonempty? Can we even find representatives of each region which are simply connected or have any specified fundamental group? 
- Not all smooth even-dimensional manifolds are almost complex. For example, $S^{4}, S^{8}, S^{10}$, etc., are not almost complex.

- If $M$ is both symplectic and complex, is it necessarily Kähler?

No. For some time, it had been suspected that every compact symplectic manifold might have an underlying Kähler structure, or, at least, that a compact symplectic manifold might have to satisfy the Hodge relations on its Betti numbers [52]. The following example first demonstrated otherwise.

\section{The Kodaira-Thurston example (Thurston, 1976 [101]):}

Take $\mathbb{R}^{4}$ with $d x_{1} \wedge d y_{1}+d x_{2} \wedge d y_{2}$, and $\Gamma$ the discrete group generated by the following symplectomorphisms:

$$
\begin{aligned}
& \gamma_{1}:\left(x_{1}, x_{2}, y_{1}, y_{2}\right) \longmapsto\left(x_{1}, x_{2}+1, y_{1}, y_{2}\right) \\
& \gamma_{2}:\left(x_{1}, x_{2}, y_{1}, y_{2}\right) \longmapsto\left(x_{1}, x_{2}, y_{1}, y_{2}+1\right) \\
& \gamma_{3}:\left(x_{1}, x_{2}, y_{1}, y_{2}\right) \longmapsto\left(x_{1}+1, x_{2}, y_{1}, y_{2}\right) \\
& \gamma_{4}: \quad\left(x_{1}, x_{2}, y_{1}, y_{2}\right) \longmapsto\left(x_{1}, x_{2}+y_{2}, y_{1}+1, y_{2}\right)
\end{aligned}
$$

Then $M=\mathbb{R}^{4} / \Gamma$ is a flat 2-torus bundle over a 2-torus. Kodaira [70] had shown that $M$ has a complex structure. However, $\pi_{1}(M)=\Gamma$, hence $H^{1}\left(\mathbb{R}^{4} / \Gamma ; \mathbb{Z}\right)=\Gamma /[\Gamma, \Gamma]$ has rank $3, b^{1}=3$ is odd, so $M$ is not Kähler [101].

- Does any symplectic manifold admit some complex structure (not necessarily compatible)?

No.

(Fernández-Gotay-Gray, 1988 [37]): There are symplectic manifolds which do not admit any complex structure [37]. Their examples are circle bundles over circle bundles over a 2-torus.

$$
\begin{array}{llll}
S^{1} & \hookrightarrow & M \\
& & \downarrow & \\
S^{1} & \hookrightarrow & P \\
& & & \downarrow \\
& & \mathbb{T}^{2} &
\end{array} \quad \text { tower of circle fibrations }
$$

- Given a complex structure on $M$, is there always a symplectic structure (not necessarily compatible)?

No.

The Hopf surface $S^{1} \times S^{3}$ is not symplectic because $H^{2}\left(S^{1} \times S^{3}\right)=0$. But it is complex since $S^{1} \times S^{3} \simeq \mathbb{C}^{2} \backslash\{0\} / \Gamma$ where $\Gamma=\left\{2^{n} \operatorname{Id} \mid n \in \mathbb{Z}\right\}$ is a group of complex transformations, i.e., we factor $\mathbb{C}^{2} \backslash\{0\}$ by the equivalence relation $\left(z_{1}, z_{2}\right) \sim\left(2 z_{1}, 2 z_{2}\right)$. 
- Is any almost complex manifold either complex or symplectic?

No.

$\mathbb{C P}^{2} \# \mathbb{C P}^{2} \# \mathbb{C P}^{2}$ is almost complex (proved by a computation with characteristic classes), but is neither complex (since it does not fit Kodaira's classification of complex surfaces), nor symplectic (as shown by Taubes [97] in 1995 using Seiberg-Witten invariants).

- In 1993 Gompf [46] provided a construction that yields a compact symplectic 4-manifold with fundamental group equal to any given finitely-presented group. In particular, we can find simply connected examples. His construction can be adapted to produce nonKähler examples.

\subsection{Main Kähler Manifolds}

\section{- Compact Riemann surfaces}

As real manifolds, these are the 2-dimensional compact orientable manifolds classified by genus. An area form is a symplectic form. Any compatible almost complex structure is always integrable for dimension reasons (see Homework 10).

\section{- Stein manifolds}

Definition 17.4 A Stein manifold is a Kähler manifold $(M, \omega)$ which admits a global proper Kähler potential, i.e., $\omega=\frac{i}{2} \partial \bar{\partial} \rho$ for some proper function $\rho: M \rightarrow \mathbb{R}$.

Proper means that the preimage by $\rho$ of a compact set is compact, i.e., " $\rho(p) \rightarrow \infty$ as $p \rightarrow \infty$."

Stein manifolds can be also characterized as the properly embedded analytic submanifolds of $\mathbb{C}^{n}$.

\section{- Complex tori}

Complex tori look like $M=\mathbb{C}^{n} / \mathbb{Z}^{n}$ where $\mathbb{Z}^{n}$ is a lattice in $\mathbb{C}^{n}$. The form $\omega=\sum d z_{j} \wedge d \bar{z}_{j}$ induced by the euclidean structure is Kähler.

\section{- Complex projective spaces}

The standard Kähler form on $\mathbb{C P}^{n}$ is the Fubini-Study form (see Homework 12 ). (In 1995 , Taubes showed that $\mathbb{C P}^{2}$ has a unique symplectic structure up to symplectomorphism.)

- Products of Kähler manifolds

- Complex submanifolds of Kähler manifolds 



\section{Part VII}

\section{Hamiltonian Mechanics}

The equations of motion in classical mechanics arise as solutions of variational problems. For a general mechanical system of $n$ particles in $\mathbb{R}^{3}$, the physical path satisfies Newton's second law. On the other hand, the physical path minimizes the mean value of kinetic minus potential energy. This quantity is called the action. For a system with constraints, the physical path is the path which minimizes the action among all paths satisfying the constraint.

The Legendre transform (Lecture 20) gives the relation between the variational (Euler-Lagrange) and the symplectic (Hamilton-Jacobi) formulations of the equations of motion.

\section{Hamiltonian Vector Fields}

\subsection{Hamiltonian and Symplectic Vector Fields}

- What does a symplectic geometer do with a real function?...

Let $(M, \omega)$ be a symplectic manifold and let $H: M \rightarrow \mathbb{R}$ be a smooth function. Its differential $d H$ is a 1 -form. By nondegeneracy, there is a unique vector field $X_{H}$ on $M$ such that $\imath_{X_{H}} \omega=d H$. Integrate $X_{H}$. Supposing that $M$ is compact, or at least that $X_{H}$ is complete, let $\rho_{t}: M \rightarrow M, t \in \mathbb{R}$, be the one-parameter family of diffeomorphisms generated by $X_{H}$ :

$$
\left\{\begin{array}{l}
\rho_{0}=\operatorname{id}_{M} \\
\frac{d \rho_{t}}{d t} \circ \rho_{t}^{-1}=X_{H} .
\end{array}\right.
$$

Claim. Each diffeomorphism $\rho_{t}$ preserves $\omega$, i.e., $\rho_{t}^{*} \omega=\omega, \forall t$.

Proof. We have $\frac{d}{d t} \rho_{t}^{*} \omega=\rho_{t}^{*} \mathcal{L}_{X_{H}} \omega=\rho_{t}^{*}(\underbrace{\imath_{X_{H}} \omega}_{d H}+\imath_{X_{H}} \underbrace{d \omega}_{0})=0$.

Therefore, every function on $(M, \omega)$ gives a family of symplectomorphisms. Notice how the proof involved both the nondegeneracy and the closedness of $\omega$.

Definition 18.1 $A$ vector field $X_{H}$ as above is called the hamiltonian vector field with hamiltonian function $H$.

Example. The height function $H(\theta, h)=h$ on the sphere $(M, \omega)=\left(S^{2}, d \theta \wedge d h\right)$ has

$$
\imath_{X_{H}}(d \theta \wedge d h)=d h \quad \Longleftrightarrow \quad X_{H}=\frac{\partial}{\partial \theta} .
$$


Thus, $\rho_{t}(\theta, h)=(\theta+t, h)$, which is rotation about the vertical axis; the height function $H$ is preserved by this motion.

Exercise. Let $X$ be a vector field on an abstract manifold $W$. There is a unique vector field $X_{\sharp}$ on the cotangent bundle $T^{*} W$, whose flow is the lift of the flow of $X$; cf. Lecture 2. Let $\alpha$ be the tautological 1 -form on $T^{*} W$ and let $\omega=-d \alpha$ be the canonical symplectic form on $T^{*} W$. Show that $X_{\sharp}$ is a hamiltonian vector field with hamiltonian function $H:=\imath_{X_{\sharp}} \alpha$.

Remark. If $X_{H}$ is hamiltonian, then

$$
\mathcal{L}_{X_{H}} H=\imath_{X_{H}} d H=\imath_{X_{H}} \imath_{X_{H}} \omega=0
$$

Therefore, hamiltonian vector fields preserve their hamiltonian functions, and each integral curve $\left\{\rho_{t}(x) \mid t \in \mathbb{R}\right\}$ of $X_{H}$ must be contained in a level set of $H$ :

$$
H(x)=\left(\rho_{t}^{*} H\right)(x)=H\left(\rho_{t}(x)\right), \quad \forall t .
$$

Definition 18.2 $A$ vector field $X$ on $M$ preserving $\omega$ (i.e., such that $\mathcal{L}_{X} \omega=0$ ) is called a symplectic vector field.

$$
\left\{\begin{array}{l}
X \text { is symplectic } \quad \Longleftrightarrow \imath_{X} \omega \text { is closed }, \\
X \text { is hamiltonian } \Longleftrightarrow \imath_{X} \omega \text { is exact . }
\end{array}\right.
$$

Locally, on every contractible open set, every symplectic vector field is hamiltonian. If $H_{\text {deRham }}^{1}(M)=0$, then globally every symplectic vector field is hamiltonian. In general, $H_{\text {deRham }}^{1}(M)$ measures the obstruction for symplectic vector fields to be hamiltonian.

Example. On the 2-torus $(M, \omega)=\left(\mathbb{T}^{2}, d \theta_{1} \wedge d \theta_{2}\right)$, the vector fields $X_{1}=\frac{\partial}{\partial \theta_{1}}$ and $X_{2}=\frac{\partial}{\partial \theta_{2}}$ are symplectic but not hamiltonian.

To summarize, vector fields on a symplectic manifold $(M, \omega)$ which preserve $\omega$ are called symplectic. The following are equivalent:

- $X$ is a symplectic vector field;

- the flow $\rho_{t}$ of $X$ preserves $\omega$, i.e., $\rho_{t}^{*} \omega=\omega$, for all $t$;

- $\mathcal{L}_{X} \omega=0$;

- $\imath_{X} \omega$ is closed.

A hamiltonian vector field is a vector field $X$ for which

- $\imath_{X} \omega$ is exact,

i.e., $\imath_{X} \omega=d H$ for some $H \in C^{\infty}(M)$. A primitive $H$ of $\imath_{X} \omega$ is then called a hamiltonian function of $X$. 


\subsection{Classical Mechanics}

Consider euclidean space $\mathbb{R}^{2 n}$ with coordinates $\left(q_{1}, \ldots, q_{n}, p_{1}, \ldots, p_{n}\right)$ and $\omega_{0}=$ $\sum d q_{j} \wedge d p_{j}$. The curve $\rho_{t}=(q(t), p(t))$ is an integral curve for $X_{H}$ exactly if

$$
\left\{\begin{aligned}
\frac{d q_{i}}{d t}(t) & =\frac{\partial H}{\partial p_{i}} \\
\frac{d p_{i}}{d t}(t) & =-\frac{\partial H}{\partial q_{i}}
\end{aligned} \quad\right. \text { (Hamilton equations) }
$$

Indeed, let $X_{H}=\sum_{i=1}^{n}\left(\frac{\partial H}{\partial p_{i}} \frac{\partial}{\partial q_{i}}-\frac{\partial H}{\partial q_{i}} \frac{\partial}{\partial p_{i}}\right)$. Then,

$$
\begin{aligned}
\imath_{X_{H}} \omega & =\sum_{j=1}^{n} \imath_{X_{H}}\left(d q_{j} \wedge d p_{j}\right)=\sum_{j=1}^{n}\left[\left(\imath_{X_{H}} d q_{j}\right) \wedge d p_{j}-d q_{j} \wedge\left(\imath_{X_{H}} d p_{j}\right)\right] \\
& =\sum_{j=1}^{n}\left(\frac{\partial H}{\partial p_{j}} d p_{j}+\frac{\partial H}{\partial q_{j}} d q_{j}\right)=d H .
\end{aligned}
$$

Remark. The gradient vector field of $H$ relative to the euclidean metric is

$$
\nabla H:=\sum_{i=1}^{n}\left(\frac{\partial H}{\partial q_{i}} \frac{\partial}{\partial q_{i}}+\frac{\partial H}{\partial p_{i}} \frac{\partial}{\partial p_{i}}\right)
$$

If $J$ is the standard (almost) complex structure so that $J\left(\frac{\partial}{\partial q_{i}}\right)=\frac{\partial}{\partial p_{i}}$ and $J\left(\frac{\partial}{\partial p_{i}}\right)=-\frac{\partial}{\partial q_{i}}$, we have $J X_{H}=\nabla H$.

The case where $n=3$ has a simple physical illustration. Newton's second law states that a particle of mass $m$ moving in configuration space $\mathbb{R}^{3}$ with coordinates $q=\left(q_{1}, q_{2}, q_{3}\right)$ under a potential $V(q)$ moves along a curve $q(t)$ such that

$$
m \frac{d^{2} q}{d t^{2}}=-\nabla V(q)
$$

Introduce the momenta $p_{i}=m \frac{d q_{i}}{d t}$ for $i=1,2,3$, and energy function $H(p, q)=$ $\frac{1}{2 m}|p|^{2}+V(q)$. Let $\mathbb{R}^{6}=T^{*} \mathbb{R}^{3}$ be the corresponding phase space, with coordinates $\left(q_{1}, q_{2}, q_{3}, p_{1}, p_{2}, p_{3}\right)$. Newton's second law in $\mathbb{R}^{3}$ is equivalent to the Hamilton equations in $\mathbb{R}^{6}$ :

$$
\left\{\begin{array}{l}
\frac{d q_{i}}{d t}=\frac{1}{m} p_{i}=\frac{\partial H}{\partial p_{i}} \\
\frac{d p_{i}}{d t}=m \frac{d^{2} q_{i}}{d t^{2}}=-\frac{\partial V}{\partial q_{i}}=-\frac{\partial H}{\partial q_{i}} .
\end{array}\right.
$$

The energy $H$ is conserved by the physical motion. 


\subsection{Brackets}

Vector fields are differential operators on functions: if $X$ is a vector field and $f \in C^{\infty}(M), d f$ being the corresponding 1-form, then

$$
X \cdot f:=d f(X)=\mathcal{L}_{X} f .
$$

Given two vector fields $X, Y$, there is a unique vector field $W$ such that

$$
\mathcal{L}_{W} f=\mathcal{L}_{X}\left(\mathcal{L}_{Y} f\right)-\mathcal{L}_{Y}\left(\mathcal{L}_{X} f\right) .
$$

The vector field $W$ is called the Lie bracket of the vector fields $X$ and $Y$ and denoted $W=[X, Y]$, since $\mathcal{L}_{W}=\left[\mathcal{L}_{X}, \mathcal{L}_{Y}\right]$ is the commutator.

Exercise. Check that, for any form $\alpha$,

$$
\imath_{[X, Y]} \alpha=\mathcal{L}_{X} \imath_{Y} \alpha-\imath_{Y} \mathcal{L}_{X} \alpha=\left[\mathcal{L}_{X}, \imath_{Y}\right] \alpha .
$$

Since each side is an anti-derivation with respect to the wedge product, it suffices to check this formula on local generators of the exterior algebra of forms, namely functions and exact 1 -forms.

Proposition 18.3 If $X$ and $Y$ are symplectic vector fields on a symplectic manifold $(M, \omega)$, then $[X, Y]$ is hamiltonian with hamiltonian function $\omega(Y, X)$.

Proof.

$$
\begin{aligned}
\imath_{[X, Y]} \omega & =\mathcal{L}_{X} \imath_{Y} \omega-\imath_{Y} \mathcal{L}_{X} \omega \\
& =d \imath_{X} \imath_{Y} \omega+\imath_{X} \underbrace{d \imath_{Y} \omega}_{0}-\imath_{Y} \underbrace{d \imath_{X} \omega}_{0}-\imath_{Y} \imath_{X} \underbrace{d \omega}_{0} \\
& =d(\omega(Y, X)) .
\end{aligned}
$$

A (real) Lie algebra is a (real) vector space $\mathfrak{g}$ together with a Lie bracket $[\cdot, \cdot]$, i.e., a bilinear map $[\cdot, \cdot]: \mathfrak{g} \times \mathfrak{g} \rightarrow \mathfrak{g}$ satisfying:

(a) $[x, y]=-[y, x], \quad \forall x, y \in \mathfrak{g}$, (antisymmetry)

(b) $[x,[y, z]]+[y,[z, x]]+[z,[x, y]]=0, \quad \forall x, y, z \in \mathfrak{g}$. (Jacobi identity)

Let

$$
\begin{aligned}
\chi(M) & =\{\text { vector fields on } M\} \\
\chi^{\text {sympl }}(M) & =\{\text { symplectic vector fields on } M\} \\
\chi^{\text {ham }}(M) & =\{\text { hamiltonian vector fields on } M\} .
\end{aligned}
$$

Corollary 18.4 The inclusions $\left(\chi^{\operatorname{ham}}(M),[\cdot, \cdot]\right) \subseteq\left(\chi^{\operatorname{sympl}}(M),[\cdot, \cdot]\right) \subseteq(\chi(M),[\cdot, \cdot])$ are inclusions of Lie algebras.

Definition 18.5 The Poisson bracket of two functions $f, g \in C^{\infty}(M ; \mathbb{R})$ is

$$
\{f, g\}:=\omega\left(X_{f}, X_{g}\right) .
$$


We have $X_{\{f, g\}}=-\left[X_{f}, X_{g}\right]$ because $X_{\omega\left(X_{f}, X_{g}\right)}=\left[X_{g}, X_{f}\right]$.

Theorem 18.6 The bracket $\{\cdot, \cdot\}$ satisfies the Jacobi identity, i.e.,

$$
\{f,\{g, h\}\}+\{g,\{h, f\}\}+\{h,\{f, g\}\}=0 .
$$

Proof. Exercise.

Definition 18.7 A Poisson algebra $(\mathcal{P},\{\cdot, \cdot\})$ is a commutative associative algebra $\mathcal{P}$ with a Lie bracket $\{\cdot, \cdot\}$ satisfying the Leibniz rule:

$$
\{f, g h\}=\{f, g\} h+g\{f, h\} .
$$

Exercise. Check that the Poisson bracket $\{\cdot, \cdot\}$ defined above satisfies the Leibniz rule.

We conclude that, if $(M, \omega)$ is a symplectic manifold, then $\left(C^{\infty}(M),\{\cdot, \cdot\}\right)$ is a Poisson algebra. Furthermore, we have a Lie algebra anti-homomorphism

$$
\begin{aligned}
& C^{\infty}(M) \longrightarrow \chi(M) \\
& H \longmapsto X_{H}
\end{aligned}
$$

\subsection{Integrable Systems}

Definition 18.8 $A$ hamiltonian system is a triple $(M, \omega, H)$, where $(M, \omega)$ is a symplectic manifold and $H \in C^{\infty}(M ; \mathbb{R})$ is a function, called the hamiltonian function.

Theorem 18.9 We have $\{f, H\}=0$ if and only if $f$ is constant along integral curves of $X_{H}$.

Proof. Let $\rho_{t}$ be the flow of $X_{H}$. Then

$$
\begin{aligned}
\frac{d}{d t}\left(f \circ \rho_{t}\right) & =\rho_{t}^{*} \mathcal{L}_{X_{H}} f=\rho_{t}^{*} \imath_{X_{H}} d f=\rho_{t}^{*} \imath_{X_{H}} \imath_{X_{f}} \omega \\
& =\rho_{t}^{*} \omega\left(X_{f}, X_{H}\right)=\rho_{t}^{*}\{f, H\} .
\end{aligned}
$$

A function $f$ as in Theorem 18.9 is called an integral of motion (or a first integral or a constant of motion). In general, hamiltonian systems do not admit integrals of motion which are independent of the hamiltonian function. Functions $f_{1}, \ldots, f_{n}$ on $M$ are said to be independent if their differentials $\left(d f_{1}\right)_{p}, \ldots,\left(d f_{n}\right)_{p}$ are linearly independent at all points $p$ in some open dense subset of $M$. Loosely speaking, a hamiltonian system is (completely) integrable if it has as many commuting integrals of motion as possible. Commutativity is with respect to the Poisson 
bracket. Notice that, if $f_{1}, \ldots, f_{n}$ are commuting integrals of motion for a hamiltonian system $(M, \omega, H)$, then, at each $p \in M$, their hamiltonian vector fields generate an isotropic subspace of $T_{p} M$ :

$$
\omega\left(X_{f_{i}}, X_{f_{j}}\right)=\left\{f_{i}, f_{j}\right\}=0 .
$$

If $f_{1}, \ldots, f_{n}$ are independent, then, by symplectic linear algebra, $n$ can be at most half the dimension of $M$.

Definition 18.10 A hamiltonian system $(M, \omega, H)$ is (completely) integrable if it possesses $n=\frac{1}{2} \operatorname{dim} M$ independent integrals of motion, $f_{1}=H, f_{2}, \ldots, f_{n}$, which are pairwise in involution with respect to the Poisson bracket, i.e., $\left\{f_{i}, f_{j}\right\}$ $=0$, for all $i, j$.

Example. The simple pendulum (Homework 13) and the harmonic oscillator are trivially integrable systems - any 2-dimensional hamiltonian system (where the set of non-fixed points is dense) is integrable.

Example. A hamiltonian system $(M, \omega, H)$ where $M$ is 4-dimensional is integrable if there is an integral of motion independent of $H$ (the commutativity condition is automatically satisfied). Homework 18 shows that the spherical pendulum is integrable.

For sophisticated examples of integrable systems, see $[10,62]$.

Let $(M, \omega, H)$ be an integrable system of dimension $2 n$ with integrals of motion $f_{1}=H, f_{2}, \ldots, f_{n}$. Let $c \in \mathbb{R}^{n}$ be a regular value of $f:=\left(f_{1}, \ldots, f_{n}\right)$. The corresponding level set, $f^{-1}(c)$, is a lagrangian submanifold, because it is $n$-dimensional and its tangent bundle is isotropic.

Lemma 18.11 If the hamiltonian vector fields $X_{f_{1}}, \ldots, X_{f_{n}}$ are complete on the level $f^{-1}(c)$, then the connected components of $f^{-1}(c)$ are homogeneous spaces for $\mathbb{R}^{n}$, i.e., are of the form $\mathbb{R}^{n-k} \times \mathbb{T}^{k}$ for some $k, 0 \leq k \leq n$, where $\mathbb{T}^{k}$ is a $k$-dimensional torus.

Proof. Exercise (just follow the flows to obtain coordinates).

Any compact component of $f^{-1}(c)$ must hence be a torus. These components, when they exist, are called Liouville tori. (The easiest way to ensure that compact components exist is to have one of the $f_{i}$ 's proper.)

Theorem 18.12 (Arnold-Liouville [3]) Let $(M, \omega, H)$ be an integrable system of dimension $2 n$ with integrals of motion $f_{1}=H, f_{2}, \ldots, f_{n}$. Let $c \in \mathbb{R}^{n}$ be a regular value of $f:=\left(f_{1}, \ldots, f_{n}\right)$. The corresponding level $f^{-1}(c)$ is a lagrangian submanifold of $M$.

(a) If the flows of $X_{f_{1}}, \ldots, X_{f_{n}}$ starting at a point $p \in f^{-1}(c)$ are complete, then the connected component of $f^{-1}(c)$ containing $p$ is a homogeneous space for $\mathbb{R}^{n}$. With respect to this affine structure, that component has coordinates $\varphi_{1}, \ldots, \varphi_{n}$, known as angle coordinates, in which the flows of the vector fields $X_{f_{1}}, \ldots, X_{f_{n}}$ are linear. 
(b) There are coordinates $\psi_{1}, \ldots, \psi_{n}$, known as action coordinates, complementary to the angle coordinates such that the $\psi_{i}$ 's are integrals of motion and $\varphi_{1}, \ldots, \varphi_{n}, \psi_{1}, \ldots, \psi_{n}$ form a Darboux chart.

Therefore, the dynamics of an integrable system is extremely simple and the system has an explicit solution in action-angle coordinates. The proof of part (a) the easy part - of the Arnold-Liouville theorem is sketched above. For the proof of part (b), see $[3,28]$.

Geometrically, regular levels being lagrangian submanifolds implies that, in a neighborhood of a regular value, the map $f: M \rightarrow \mathbb{R}^{n}$ collecting the given integrals of motion is a lagrangian fibration, i.e., it is locally trivial and its fibers are lagrangian submanifolds. Part (a) of the Arnold-Liouville theorem states that there are coordinates along the fibers, the angle coordinates $\varphi_{i}{ }^{11}$ in which the flows of $X_{f_{1}}, \ldots, X_{f_{n}}$ are linear. Part (b) of the theorem guarantees the existence of coordinates on $\mathbb{R}^{n}$, the action coordinates $\psi_{i}$, which (Poisson) commute among themselves and satisfy $\left\{\varphi_{i}, \psi_{j}\right\}=\delta_{i j}$ with respect to the angle coordinates. Notice that, in general, the action coordinates are not the given integrals of motion because $\varphi_{1}, \ldots, \varphi_{n}, f_{1}, \ldots, f_{n}$ do not form a Darboux chart.

\footnotetext{
${ }^{11}$ The name "angle coordinates" is used even if the fibers are not tori.
} 


\section{Homework 13: Simple Pendulum}

This problem is adapted from [53].

The simple pendulum is a mechanical system consisting of a massless rigid rod of length $l$, fixed at one end, whereas the other end has a plumb bob of mass $m$, which may oscillate in the vertical plane. Assume that the force of gravity is constant pointing vertically downwards, and that this is the only external force acting on this system.

(a) Let $\theta$ be the oriented angle between the rod (regarded as a line segment) and the vertical direction. Let $\xi$ be the coordinate along the fibers of $T^{*} S^{1}$ induced by the standard angle coordinate on $S^{1}$. Show that the function $H: T^{*} S^{1} \rightarrow \mathbb{R}$ given by

$$
H(\theta, \xi)=\underbrace{\frac{\xi^{2}}{2 m l^{2}}}_{K}+\underbrace{m l(1-\cos \theta)}_{V},
$$

is an appropriate hamiltonian function to describe the simple pendulum. More precisely, check that gravity corresponds to the potential energy $V(\theta)=$ $m l(1-\cos \theta)$ (we omit universal constants), and that the kinetic energy is given by $K(\theta, \xi)=\frac{1}{2 m l^{2}} \xi^{2}$.

(b) For simplicity assume that $m=l=1$.

Plot the level curves of $H$ in the $(\theta, \xi)$ plane.

Show that there exists a number $c$ such that for $0<h<c$ the level curve $H=h$ is a disjoint union of closed curves. Show that the projection of each of these curves onto the $\theta$-axis is an interval of length less than $\pi$.

Show that neither of these assertions is true if $h>c$.

What types of motion are described by these two types of curves?

What about the case $H=c$ ?

(c) Compute the critical points of the function $H$. Show that, modulo $2 \pi$ in $\theta$, there are exactly two critical points: a critical point $s$ where $H$ vanishes, and a critical point $u$ where $H$ equals $c$. These points are called the stable and unstable points of $H$, respectively. Justify this terminology, i.e., show that a trajectory of the hamiltonian vector field of $H$ whose initial point is close to $s$ stays close to $s$ forever, and show that this is not the case for $u$. What is happening physically? 


\section{Variational Principles}

\subsection{Equations of Motion}

The equations of motion in classical mechanics arise as solutions of variational problems:

A general mechanical system possesses both kinetic and potential energy. The quantity that is minimized is the mean value of kinetic minus potential energy.

Example. Suppose that a point-particle of mass $m$ moves in $\mathbb{R}^{3}$ under a force field $F$; let $x(t), a \leq t \leq b$, be its path of motion in $\mathbb{R}^{3}$. Newton's second law states that

$$
m \frac{d^{2} x}{d t^{2}}(t)=F(x(t)) .
$$

Define the work of a path $\gamma:[a, b] \longrightarrow \mathbb{R}^{3}$, with $\gamma(a)=p$ and $\gamma(b)=q$, to be

$$
W_{\gamma}=\int_{a}^{b} F(\gamma(t)) \cdot \frac{d \gamma}{d t}(t) d t
$$

Suppose that $F$ is conservative, i.e., $W_{\gamma}$ depends only on $p$ and $q$. Then we can define the potential energy $V: \mathbb{R}^{3} \longrightarrow \mathbb{R}$ of the system as

$$
V(q):=W_{\gamma}
$$

where $\gamma$ is a path joining a fixed base point $p_{0} \in \mathbb{R}^{3}$ (the "origin") to $q$. Newton's second law can now be written

$$
m \frac{d^{2} x}{d t^{2}}(t)=-\frac{\partial V}{\partial x}(x(t)) .
$$

In the previous lecture we saw that

$$
\begin{array}{lll}
\text { Newton's second law } & \Longleftrightarrow & \text { Hamilton equations } \\
\text { in } \mathbb{R}^{3}=\left\{\left(q_{1}, q_{2}, q_{3}\right)\right\} & \text { in } T^{*} \mathbb{R}^{3}=\left\{\left(q_{1}, q_{2}, q_{3}, p_{1}, p_{2}, p_{3}\right)\right\}
\end{array}
$$

where $p_{i}=m \frac{d q_{i}}{d t}$ and the hamiltonian is $H(p, q)=\frac{1}{2 m}|p|^{2}+V(q)$. Hence, solving Newton's second law in configuration space $\mathbb{R}^{3}$ is equivalent to solving in phase space $T^{*} \mathbb{R}^{3}$ for the integral curve of the hamiltonian vector field with hamiltonian function $H$.

Example. The motion of earth about the sun, both regarded as point-masses and assuming that the sun to be stationary at the origin, obeys the inverse square law

$$
m \frac{d^{2} x}{d t^{2}}=-\frac{\partial V}{\partial x},
$$

where $x(t)$ is the position of earth at time $t$, and $V(x)=\frac{\text { const. is the gravitational }}{|x|}$ potential. 


\subsection{Principle of Least Action}

When we need to deal with systems with constraints, such as the simple pendulum, or two point masses attached by a rigid rod, or a rigid body, the language of variational principles becomes more appropriate than the explicit analogues of Newton's second laws. Variational principles are due mostly to D'Alembert, Maupertius, Euler and Lagrange.

Example. (The n-particle system.) Suppose that we have $n$ point-particles of masses $m_{1}, \ldots, m_{n}$ moving in 3 -space. At any time $t$, the configuration of this system is described by a vector in configuration space $\mathbb{R}^{3 n}$

$$
x=\left(x_{1}, \ldots, x_{n}\right) \in \mathbb{R}^{3 n}
$$

with $x_{i} \in \mathbb{R}^{3}$ describing the position of the $i$ th particle. If $V \in C^{\infty}\left(\mathbb{R}^{3 n}\right)$ is the potential energy, then a path of motion $x(t), a \leq t \leq b$, satisfies

$$
m_{i} \frac{d^{2} x_{i}}{d t^{2}}(t)=-\frac{\partial V}{\partial x_{i}}\left(x_{1}(t), \ldots, x_{n}(t)\right) .
$$

Consider this path in configuration space as a map $\gamma_{0}:[a, b] \rightarrow \mathbb{R}^{3 n}$ with $\gamma_{0}(a)=p$ and $\gamma_{0}(b)=q$, and let

$$
\mathcal{P}=\left\{\gamma:[a, b] \longrightarrow \mathbb{R}^{3 n} \mid \gamma(a)=p \text { and } \gamma(b)=q\right\}
$$

be the set of all paths going from $p$ to $q$ over time $t \in[a, b]$.

Definition 19.1 The action of a path $\gamma \in \mathcal{P}$ is

$$
\mathcal{A}_{\gamma}:=\int_{a}^{b}\left(\sum_{i=1}^{n} \frac{m_{i}}{2}\left|\frac{d \gamma_{i}}{d t}(t)\right|^{2}-V(\gamma(t))\right) d t
$$

\section{Principle of least action.}

The physical path $\gamma_{0}$ is the path for which $\mathcal{A}_{\gamma}$ is minimal.

\section{Newton's second law for a constrained system.}

Suppose that the $n$ point-masses are restricted to move on a submanifold $M$ of $\mathbb{R}^{3 n}$ called the constraint set. We can now single out the actual physical path $\gamma_{0}:[a, b] \rightarrow M$, with $\gamma_{0}(a)=p$ and $\gamma_{0}(b)=q$, as being "the" path which minimizes $\mathcal{A}_{\gamma}$ among all those hypothetical paths $\gamma:[a, b] \rightarrow \mathbb{R}^{3 n}$ with $\gamma(a)=p$, $\gamma(b)=q$ and satisfying the rigid constraints $\gamma(t) \in M$ for all $t$.

\subsection{Variational Problems}

Let $M$ be an $n$-dimensional manifold. Its tangent bundle $T M$ is a $2 n$-dimensional manifold. Let $F: T M \rightarrow \mathbb{R}$ be a smooth function. 
If $\gamma:[a, b] \rightarrow M$ is a smooth curve on $M$, define the lift of $\gamma$ to $T M$ to be the smooth curve on $T M$ given by

$$
\begin{aligned}
\tilde{\gamma}:[a, b] & \longrightarrow T M \\
t & \longmapsto\left(\gamma(t), \frac{d \gamma}{d t}(t)\right) .
\end{aligned}
$$

The action of $\gamma$ is

$$
\mathcal{A}_{\gamma}:=\int_{a}^{b}\left(\tilde{\gamma}^{*} F\right)(t) d t=\int_{a}^{b} F\left(\gamma(t), \frac{d \gamma}{d t}(t)\right) d t
$$

For fixed $p, q \in M$, let

$$
\mathcal{P}(a, b, p, q):=\{\gamma:[a, b] \longrightarrow M \mid \gamma(a)=p, \gamma(b)=q\}
$$

\section{Problem.}

Find, among all $\gamma \in \mathcal{P}(a, b, p, q)$, the curve $\gamma_{0}$ which "minimizes" $\mathcal{A}_{\gamma}$.

First observe that minimizing curves are always locally minimizing:

Lemma 19.2 Suppose that $\gamma_{0}:[a, b] \rightarrow M$ is minimizing. Let $\left[a_{1}, b_{1}\right]$ be a subinterval of $[a, b]$ and let $p_{1}=\gamma_{0}\left(a_{1}\right), q_{1}=\gamma_{0}\left(b_{1}\right)$. Then $\left.\gamma_{0}\right|_{\left[a_{1}, b_{1}\right]}$ is minimizing among the curves in $\mathcal{P}\left(a_{1}, b_{1}, p_{1}, q_{1}\right)$.

Proof. Exercise:

Argue by contradiction. Suppose that there were $\gamma_{1} \in \mathcal{P}\left(a_{1}, b_{1}, p_{1}, q_{1}\right)$ for

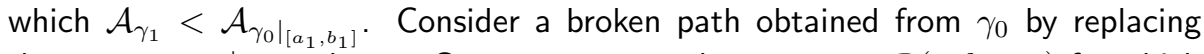
the segment $\left.\gamma_{0}\right|_{\left[a_{1}, b_{1}\right]}$ by $\gamma_{1}$. Construct a smooth curve $\gamma_{2} \in \mathcal{P}(a, b, p, q)$ for which $\mathcal{A}_{\gamma_{2}}<\mathcal{A}_{\gamma_{0}}$ by rounding off the corners of the broken path.

We will now assume that $p, q$ and $\gamma_{0}$ lie in a coordinate neighborhood $\left(\mathcal{U}, x_{1}, \ldots, x_{n}\right)$. On $T \mathcal{U}$ we have coordinates $\left(x_{1}, \ldots, x_{n}, v_{1}, \ldots, v_{n}\right)$ associated with a trivialization of $T \mathcal{U}$ by $\frac{\partial}{\partial x_{1}}, \ldots, \frac{\partial}{\partial x_{n}}$. Using this trivialization, the curve

$$
\gamma:[a, b] \longrightarrow \mathcal{U}, \quad \gamma(t)=\left(\gamma_{1}(t), \ldots, \gamma_{n}(t)\right)
$$

lifts to

$$
\tilde{\gamma}:[a, b] \longrightarrow T \mathcal{U}, \quad \tilde{\gamma}(t)=\left(\gamma_{1}(t), \ldots, \gamma_{n}(t), \frac{d \gamma_{1}}{d t}(t), \ldots, \frac{d \gamma_{n}}{d t}(t)\right)
$$

Necessary condition for $\gamma_{0} \in \mathcal{P}(a, b, p, q)$ to minimize the action.

Let $c_{1}, \ldots, c_{n} \in C^{\infty}([a, b])$ be such that $c_{i}(a)=c_{i}(b)=0$. Let $\gamma_{\varepsilon}:[a, b] \longrightarrow \mathcal{U}$ be the curve

$$
\gamma_{\varepsilon}(t)=\left(\gamma_{1}(t)+\varepsilon c_{1}(t), \ldots, \gamma_{n}(t)+\varepsilon c_{n}(t)\right) .
$$

For $\varepsilon$ small, $\gamma_{\varepsilon}$ is well-defined and in $\mathcal{P}(a, b, p, q)$. 
Let $\mathcal{A}_{\varepsilon}=\mathcal{A}_{\gamma_{\varepsilon}}=\int_{a}^{b} F\left(\gamma_{\varepsilon}(t), \frac{d \gamma_{\varepsilon}}{d t}(t)\right) d t$. If $\gamma_{0}$ minimizes $\mathcal{A}$, then

$$
\begin{gathered}
\frac{d \mathcal{A}_{\varepsilon}}{d \varepsilon}(0)=0 . \\
\frac{d \mathcal{A}_{\varepsilon}}{d \varepsilon}(0)=\int_{a}^{b} \sum_{i}\left[\frac{\partial F}{\partial x_{i}}\left(\gamma_{0}(t), \frac{d \gamma_{0}}{d t}(t)\right) c_{i}(t)+\frac{\partial F}{\partial v_{i}}\left(\gamma_{0}(t), \frac{d \gamma_{0}}{d t}(t)\right) \frac{d c_{i}}{d t}(t)\right] d t \\
=\int_{a}^{b} \sum_{i}\left[\frac{\partial F}{\partial x_{i}}(\ldots)-\frac{d}{d t} \frac{\partial F}{\partial v_{i}}(\ldots)\right] c_{i}(t) d t=0
\end{gathered}
$$

where the first equality follows from the Leibniz rule and the second equality follows from integration by parts. Since this is true for all $c_{i}$ 's satisfying the boundary conditions $c_{i}(a)=c_{i}(b)=0$, we conclude that

$$
\frac{\partial F}{\partial x_{i}}\left(\gamma_{0}(t), \frac{d \gamma_{0}}{d t}(t)\right)=\frac{d}{d t} \frac{\partial F}{\partial v_{i}}\left(\gamma_{0}(t), \frac{d \gamma_{0}}{d t}(t)\right) .
$$

E-L

These are the Euler-Lagrange equations.

\subsection{Solving the Euler-Lagrange Equations}

Case 1: Suppose that $F(x, v)$ does not depend on $v$.

The Euler-Lagrange equations become

$$
\frac{\partial F}{\partial x_{i}}\left(\gamma_{0}(t), \frac{d \gamma_{0}}{d t}(t)\right)=0 \Longleftrightarrow \text { the curve } \gamma_{0} \text { sits on the critical set of } F \text {. }
$$

For generic $F$, the critical points are isolated, hence $\gamma_{0}(t)$ must be a constant curve.

Case 2: Suppose that $F(x, v)$ depends affinely on $v$ :

$$
F(x, v)=F_{0}(x)+\sum_{j=1}^{n} F_{j}(x) v_{j} .
$$

$$
\begin{array}{cl}
\text { LHS of E-L : } & \frac{\partial F_{0}}{\partial x_{i}}(\gamma(t))+\sum_{j=1}^{n} \frac{\partial F_{j}}{\partial x_{i}}(\gamma(t)) \frac{d \gamma_{j}}{d t}(t) \\
\text { RHS of E-L : } & \frac{d}{d t} F_{i}(\gamma(t))=\sum_{j=1}^{n} \frac{\partial F_{i}}{\partial x_{j}}(\gamma(t)) \frac{d \gamma_{j}}{d t}(t)
\end{array}
$$

The Euler-Lagrange equations become

$$
\frac{\partial F_{0}}{\partial x_{i}}(\gamma(t))=\sum_{j=1}^{n} \underbrace{\left(\frac{\partial F_{i}}{\partial x_{j}}-\frac{\partial F_{j}}{\partial x_{i}}\right)}_{n \times n \text { matrix }}(\gamma(t)) \frac{d \gamma_{j}}{d t}(t) .
$$


If the $n \times n$ matrix $\left(\frac{\partial F_{i}}{\partial x_{j}}-\frac{\partial F_{j}}{\partial x_{i}}\right)$ has an inverse $G_{i j}(x)$, then

$$
\frac{d \gamma_{j}}{d t}(t)=\sum_{i=1}^{n} G_{j i}(\gamma(t)) \frac{\partial F_{0}}{\partial x_{i}}(\gamma(t))
$$

is a system of first order ordinary differential equations. Locally it has a unique solution through each point $p$. If $q$ is not on this curve, there is no solution at all to the Euler-Lagrange equations belonging to $\mathcal{P}(a, b, p, q)$.

Therefore, we need non-linear dependence of $F$ on the $v$ variables in order to have appropriate solutions. From now on, assume that the

$$
\text { Legendre condition: } \quad \operatorname{det}\left(\frac{\partial^{2} F}{\partial v_{i} \partial v_{j}}\right) \neq 0 \text {. }
$$

Letting $G_{i j}(x, v)=\left(\frac{\partial^{2} F}{\partial v_{i} \partial v_{j}}(x, v)\right)^{-1}$, the Euler-Lagrange equations become

$$
\frac{d^{2} \gamma_{j}}{d t^{2}}=\sum_{i} G_{j i} \frac{\partial F}{\partial x_{i}}\left(\gamma, \frac{d \gamma}{d t}\right)-\sum_{i, k} G_{j i} \frac{\partial^{2} F}{\partial v_{i} \partial x_{k}}\left(\gamma, \frac{d \gamma}{d t}\right) \frac{d \gamma_{k}}{d t}
$$

This second order ordinary differential equation has a unique solution given initial conditions

$$
\gamma(a)=p \quad \text { and } \quad \frac{d \gamma}{d t}(a)=v
$$

\subsection{Minimizing Properties}

Is the above solution locally minimizing?

Assume that $\left(\frac{\partial^{2} F}{\partial v_{i} \partial v_{j}}(x, v)\right) \gg 0, \forall(x, v)$, i.e., with the $x$ variable frozen, the function $v \mapsto F(x, v)$ is strictly convex.

Suppose that $\gamma_{0} \in \mathcal{P}(a, b, p, q)$ satisfies E-L. Does $\gamma_{0}$ minimize $\mathcal{A}_{\gamma}$ ? Locally, yes, according to the following theorem. (Globally it is only critical.)

Theorem 19.3 For every sufficiently small subinterval $\left[a_{1}, b_{1}\right]$ of $[a, b],\left.\gamma_{0}\right|_{\left[a_{1}, b_{1}\right]}$ is locally minimizing in $\mathcal{P}\left(a_{1}, b_{1}, p_{1}, q_{1}\right)$ where $p_{1}=\gamma_{0}\left(a_{1}\right), q_{1}=\gamma_{0}\left(b_{1}\right)$.

Proof. As an exercise in Fourier series, show the Wirtinger inequality: for $f \in$ $C^{1}([a, b])$ with $f(a)=f(b)=0$, we have

$$
\int_{a}^{b}\left|\frac{d f}{d t}\right|^{2} d t \geq \frac{\pi^{2}}{(b-a)^{2}} \int_{a}^{b}|f|^{2} d t
$$

Suppose that $\gamma_{0}:[a, b] \rightarrow \mathcal{U}$ satisfies E-L. Take $c_{i} \in C^{\infty}([a, b]), c_{i}(a)=$ $c_{i}(b)=0$. Let $c=\left(c_{1}, \ldots, c_{n}\right)$. Let $\gamma_{\varepsilon}=\gamma_{0}+\varepsilon c \in \mathcal{P}(a, b, p, q)$, and let $\mathcal{A}_{\varepsilon}=\mathcal{A}_{\gamma_{\varepsilon}}$. 
$\mathbf{E}-\mathbf{L} \Longleftrightarrow \frac{d \mathcal{A}_{\varepsilon}}{d \varepsilon}(0)=0$

$$
\begin{aligned}
\frac{d^{2} \mathcal{A}_{\varepsilon}}{d \varepsilon^{2}}(0) & =\int_{a}^{b} \sum_{i, j} \frac{\partial^{2} F}{\partial x_{i} \partial x_{j}}\left(\gamma_{0}, \frac{d \gamma_{0}}{d t}\right) c_{i} c_{j} d t \\
& +2 \int_{a}^{b} \sum_{i, j} \frac{\partial^{2} F}{\partial x_{i} \partial v_{j}}\left(\gamma_{0}, \frac{d \gamma_{0}}{d t}\right) c_{i} \frac{d c_{j}}{d t} d t \\
& +\int_{a}^{b} \sum_{i, j} \frac{\partial^{2} F}{\partial v_{i} \partial v_{j}}\left(\gamma_{0}, \frac{d \gamma_{0}}{d t}\right) \frac{d c_{i}}{d t} \frac{d c_{j}}{d t} d t
\end{aligned}
$$

Since $\left(\frac{\partial^{2} F}{\partial v_{i} \partial v_{j}}(x, v)\right) \gg 0$ at all $x, v$,

$$
\begin{aligned}
\mathrm{III} & \geq K_{\mathrm{III}}\left|\frac{d c}{d t}\right|_{L^{2}[a, b]}^{2} \\
|\mathrm{I}| & \leq K_{\mathrm{I}}|c|_{L^{2}[a, b]}^{2} \\
|\mathrm{II}| & \leq K_{\mathrm{II}}|c|_{L^{2}[a, b]}\left|\frac{d c}{d t}\right|_{L^{2}[a, b]}
\end{aligned}
$$

where $K_{\mathrm{I}}, K_{\mathrm{II}}, K_{\mathrm{III}}>0$. By the Wirtinger inequality, if $b-a$ is very small, then III $>|\mathrm{II}+| \mathrm{II} \mid$ when $c \neq 0$. Hence, $\gamma_{0}$ is a local minimum. 


\section{Homework 14: Minimizing Geodesics}

This set of problems is adapted from [53].

Let $(M, g)$ be a riemannian manifold. From the riemannian metric, we get a function $F: T M \rightarrow \mathbb{R}$, whose restriction to each tangent space $T_{p} M$ is the quadratic form defined by the metric.

Let $p$ and $q$ be points on $M$, and let $\gamma:[a, b] \rightarrow M$ be a smooth curve joining $p$ to $q$. Let $\tilde{\gamma}:[a, b] \rightarrow T M, \tilde{\gamma}(t)=\left(\gamma(t), \frac{d \gamma}{d t}(t)\right)$ be the lift of $\gamma$ to $T M$. The action of $\gamma$ is

$$
\mathcal{A}(\gamma)=\int_{a}^{b}\left(\tilde{\gamma}^{*} F\right) d t=\int_{a}^{b}\left|\frac{d \gamma}{d t}\right|^{2} d t .
$$

1. Let $\gamma:[a, b] \rightarrow M$ be a smooth curve joining $p$ to $q$. Show that the arclength of $\gamma$ is independent of the parametrization of $\gamma$, i.e., show that if we reparametrize $\gamma$ by $\tau:\left[a^{\prime}, b^{\prime}\right] \rightarrow[a, b]$, the new curve $\gamma^{\prime}=\gamma \circ \tau:\left[a^{\prime}, b^{\prime}\right] \rightarrow M$ has the same arc-length.

2. Show that, given any curve $\gamma:[a, b] \rightarrow M$ (with $\frac{d \gamma}{d t}$ never vanishing), there is a reparametrization $\tau:[a, b] \rightarrow[a, b]$ such that $\gamma \circ \tau:[a, b] \rightarrow M$ is of constant velocity, that is, $\left|\frac{d \gamma}{d t}\right|$ is independent of $t$.

3. Let $\tau:[a, b] \rightarrow[a, b]$ be a smooth monotone map taking the endpoints of $[a, b]$ to the endpoints of $[a, b]$. Prove that

$$
\int_{a}^{b}\left(\frac{d \tau}{d t}\right)^{2} d t \geq b-a
$$

with equality holding if and only if $\frac{d \tau}{d t}=1$.

4. Let $\gamma:[a, b] \rightarrow M$ be a smooth curve joining $p$ to $q$. Suppose that, as $s$ goes from $a$ to $b$, its image $\gamma(s)$ moves at constant velocity, i.e., suppose that $\left|\frac{d \gamma}{d s}\right|$ is constant as a function of $s$. Let $\gamma^{\prime}=\gamma \circ \tau:[a, b] \rightarrow M$ be a reparametrization of $\gamma$. Show that $\mathcal{A}\left(\gamma^{\prime}\right) \geq \mathcal{A}(\gamma)$, with equality holding if and only if $\tau(t) \equiv t$. 
5. Let $\gamma_{0}:[a, b] \rightarrow M$ be a curve joining $p$ to $q$. Suppose that $\gamma_{0}$ is actionminimizing, i.e., suppose that

$$
\mathcal{A}\left(\gamma_{0}\right) \leq \mathcal{A}(\gamma)
$$

for any other curve $\gamma:[a, b] \rightarrow M$ joining $p$ to $q$. Prove that $\gamma_{0}$ is also arc-length-minimizing, i.e., show that $\gamma_{0}$ is the shortest geodesic joining $p$ to $q$.

6. Show that, among all curves joining $p$ to $q, \gamma_{0}$ minimizes the action if and only if $\gamma_{0}$ is of constant velocity and $\gamma_{0}$ minimizes arc-length.

7. On a coordinate chart $\left(\mathcal{U}, x^{1}, \ldots, x^{n}\right)$ on $M$, we have

$$
F(x, v)=\sum g_{i j}(x) v^{i} v^{j} .
$$

Show that the Euler-Lagrange equations associated to the action reduce to the Christoffel equations for a geodesic

$$
\frac{d^{2} \gamma^{k}}{d t^{2}}+\sum\left(\Gamma_{i j}^{k} \circ \gamma\right) \frac{d \gamma^{i}}{d t} \frac{d \gamma^{j}}{d t}=0
$$

where the $\Gamma_{i j}^{k}$ 's (called the Christoffel symbols) are defined in terms of the coefficients of the riemannian metric by

$$
\Gamma_{i j}^{k}=\frac{1}{2} \sum_{\ell} g^{\ell k}\left(\frac{\partial g_{\ell i}}{\partial x_{j}}+\frac{\partial g_{\ell j}}{\partial x_{i}}-\frac{\partial g_{i j}}{\partial x_{\ell}}\right),
$$

$\left(g^{i j}\right)$ being the matrix inverse to $\left(g_{i j}\right)$.

8. Let $p$ and $q$ be two non-antipodal points on $S^{n}$. Show that the geodesic joining $p$ to $q$ is an arc of a great circle, the great circle in question being the intersection of $S^{n}$ with the two-dimensional subspace of $\mathbb{R}^{n+1}$ spanned by $p$ and $q$.

Hint: No calculations are needed: Show that an isometry of a riemannian manifold has to carry geodesics into geodesics, and show that there is an isometry of $\mathbb{R}^{n+1}$ whose fixed point set is the plane spanned by $p$ and $q$, and show that this isometry induces on $S^{n}$ an isometry whose fixed point set is the great circle containing $p$ and $q$. 


\section{Legendre Transform}

\subsection{Strict Convexity}

Let $V$ be an $n$-dimensional vector space, with $e_{1}, \ldots, e_{n}$ a basis of $V$ and $v_{1}, \ldots, v_{n}$ the associated coordinates. Let $F: V \rightarrow \mathbb{R}, F=F\left(v_{1}, \ldots, v_{n}\right)$, be a smooth function. Let $p \in V, u=\sum_{i=1}^{n} u_{i} e_{i} \in V$. The hessian of $F$ is the quadratic function on $V$ defined by

$$
\left(d^{2} F\right)_{p}(u):=\sum_{i, j} \frac{\partial^{2} F}{\partial v_{i} \partial v_{j}}(p) u_{i} u_{j} .
$$

Exercise. Show that $\left(d^{2} F\right)_{p}(u)=\left.\frac{d^{2}}{d t^{2}} F(p+t u)\right|_{t=0}$.

Definition 20.1 The function $F$ is strictly convex if $\left(d^{2} F\right)_{p} \gg 0, \forall p \in V$.

Proposition 20.2 For a strictly convex function $F$ on $V$, the following are equivalent:

(a) $F$ has a critical point, i.e., a point where $d F_{p}=0$;

(b) F has a local minimum at some point;

(c) F has a unique critical point (global minimum); and

(d) $F$ is proper, that is, $F(p) \rightarrow+\infty$ as $p \rightarrow \infty$ in $V$.

Proof. Homework 15.

Definition 20.3 A strictly convex function $F$ is stable when it satisfies conditions (a)-(d) in Proposition 20.2.

Example. The function $e^{x}+a x$ is strictly convex for any $a \in \mathbb{R}$, but it is stable only for $a<0$. The function $x^{2}+a x$ is strictly convex and stable for any $a \in \mathbb{R}$. $\diamond$

\subsection{Legendre Transform}

Let $F$ be any strictly convex function on $V$. Given $\ell \in V^{*}$, let

$$
F_{\ell}: V \longrightarrow \mathbb{R}, \quad F_{\ell}(v)=F(v)-\ell(v) .
$$

Since $\left(d^{2} F\right)_{p}=\left(d^{2} F_{\ell}\right)_{p}$,

$$
F \text { is strictly convex } \Longleftrightarrow F_{\ell} \text { is strictly convex. }
$$


Definition 20.4 The stability set of a strictly convex function $F$ is

$$
S_{F}=\left\{\ell \in V^{*} \mid F_{\ell} \text { is stable }\right\} .
$$

Proposition 20.5 The set $S_{F}$ is an open and convex subset of $V^{*}$.

Proof. Homework 15.

Homework 15 also describes a sufficient condition for $S_{F}=V^{*}$.

Definition 20.6 The Legendre transform associated to $F \in C^{\infty}(V ; \mathbb{R})$ is the map

$$
\begin{aligned}
L_{F}: V & \longrightarrow V^{*} \\
p & \longmapsto d F_{p} \in T_{p}^{*} V \simeq V^{*} .
\end{aligned}
$$

Proposition 20.7 Suppose that $F$ is strictly convex. Then

$$
L_{F}: V \stackrel{\simeq}{\longrightarrow} S_{F},
$$

i.e., $L_{F}$ is a diffeomorphism onto $S_{F}$.

The inverse map $L_{F}^{-1}: S_{F} \rightarrow V$ is described as follows: for $\ell \in S_{F}$, the value $L_{F}^{-1}(\ell)$ is the unique minimum point $p_{\ell} \in V$ of $F_{\ell}=F-\ell$.

Exercise. Check that $p$ is the minimum of $F(v)-d F_{p}(v)$.

Definition 20.8 The dual function $F^{*}$ to $F$ is

$$
F^{*}: S_{F} \longrightarrow \mathbb{R}, \quad F^{*}(\ell)=-\min _{p \in V} F_{\ell}(p) .
$$

Theorem 20.9 We have that $L_{F}^{-1}=L_{F^{*}}$.

Proof. Homework 15.

\subsection{Application to Variational Problems}

Let $M$ be a manifold and $F: T M \rightarrow \mathbb{R}$ a function on $T M$.

Problem. Minimize $\mathcal{A}_{\gamma}=\int \tilde{\gamma}^{*} F$.

At $p \in M$, let

$$
F_{p}:=\left.F\right|_{T_{p} M}: T_{p} M \longrightarrow \mathbb{R} .
$$

Assume that $F_{p}$ is strictly convex for all $p \in M$. To simplify notation, assume also that $S_{F_{p}}=T_{p}^{*} M$. The Legendre transform on each tangent space

$$
L_{F_{p}}: T_{p} M \stackrel{\simeq}{\longrightarrow} T_{p}^{*} M
$$


is essentially given by the first derivatives of $F$ in the $v$ directions. The dual function to $F_{p}$ is $F_{p}^{*}: T_{p}^{*} M \longrightarrow \mathbb{R}$. Collect these fiberwise maps into

$$
\begin{array}{rlrl}
\mathcal{L}: T M & \longrightarrow T^{*} M, & \left.\mathcal{L}\right|_{T_{p} M} & =L_{F_{p}}, \quad \text { and } \\
H: T^{*} M & \longrightarrow \mathbb{R}, & \left.H\right|_{T_{p}^{*} M}=F_{p}^{*} .
\end{array}
$$

Exercise. The maps $H$ and $\mathcal{L}$ are smooth, and $\mathcal{L}$ is a diffeomorphism.

Let

$$
\begin{array}{ll}
\gamma:[a, b] \longrightarrow M & \text { be a curve, } \\
\tilde{\gamma}:[a, b] \longrightarrow T M & \text { its lift. }
\end{array}
$$

Theorem 20.10 The curve $\gamma$ satisfies the Euler-Lagrange equations on every coordinate chart if and only if $\mathcal{L} \circ \tilde{\gamma}:[a, b] \rightarrow T^{*} M$ is an integral curve of the hamiltonian vector field $X_{H}$.

Proof. Let

$$
\begin{aligned}
& \left(\mathcal{U}, x_{1}, \ldots, x_{n}\right) \quad \text { coordinate neighborhood in } M \\
& \left(T \mathcal{U}, x_{1}, \ldots, x_{n}, v_{1}, \ldots, v_{n}\right) \quad \text { coordinates in } T M \\
& \left(T^{*} \mathcal{U}, x_{1}, \ldots, x_{n}, \xi_{1}, \ldots, \xi_{n}\right) \quad \text { coordinates in } T^{*} M
\end{aligned}
$$

On $T \mathcal{U}$ we have $F=F(x, v)$.

On $T^{*} \mathcal{U}$ we have $H=H(u, \xi)$.

$$
\begin{aligned}
\mathcal{L}: \quad T \mathcal{U} & \longrightarrow T^{*} \mathcal{U} \\
(x, v) & \longmapsto(x, \xi) \quad \text { where } \quad \xi=L_{F_{x}}(v)=\frac{\partial F}{\partial v}(x, v) .
\end{aligned}
$$

(This is the definition of momentum $\xi$.)

$$
H(x, \xi)=F_{x}^{*}(\xi)=\xi \cdot v-F(x, v) \quad \text { where } \quad \mathcal{L}(x, v)=(x, \xi) .
$$

Integral curves $(x(t), \xi(t))$ of $X_{H}$ satisfy the Hamilton equations:

$$
\mathbf{H}\left\{\begin{aligned}
\frac{d x}{d t} & =\frac{\partial H}{\partial \xi}(x, \xi) \\
\frac{d \xi}{d t} & =-\frac{\partial H}{\partial x}(x, \xi),
\end{aligned}\right.
$$

whereas the physical path $x(t)$ satisfies the Euler-Lagrange equations:

$$
\text { E-L } \quad \frac{\partial F}{\partial x}\left(x, \frac{d x}{d t}\right)=\frac{d}{d t} \frac{\partial F}{\partial v}\left(x, \frac{d x}{d t}\right) .
$$

Let $(x(t), \xi(t))=\mathcal{L}\left(x(t), \frac{d x}{d t}(t)\right)$. We want to prove:

$$
t \mapsto(x(t), \xi(t)) \text { satisfies } \mathbf{H} \quad \Longleftrightarrow \quad t \mapsto\left(x(t), \frac{d x}{d t}(t)\right) \text { satisfies } \mathbf{E}-\mathbf{L} \text {. }
$$


The first line of $\mathbf{H}$ is automatically satisfied:

$$
\frac{d x}{d t}=\frac{\partial H}{\partial \xi}(x, \xi)=L_{F_{x}^{*}}(\xi)=L_{F_{x}}^{-1}(\xi) \quad \Longleftrightarrow \quad \xi=L_{F_{x}}\left(\frac{d x}{d t}\right)
$$

Claim. If $(x, \xi)=\mathcal{L}(x, v)$, then $\frac{\partial F}{\partial x}(x, v)=-\frac{\partial H}{\partial x}(x, \xi)$.

This follows from differentiating both sides of $H(x, \xi)=\xi \cdot v-F(x, v)$ with respect to $x$, where $\xi=L_{F_{x}}(v)=\xi(x, v)$.

$$
\frac{\partial H}{\partial x}+\underbrace{\frac{\partial H}{\partial \xi}}_{v} \frac{\partial \xi}{\partial x}=\frac{\partial \xi}{\partial x} \cdot v-\frac{\partial F}{\partial x} .
$$

Now the second line of $\mathbf{H}$ becomes

$$
\underbrace{\frac{d}{d t} \frac{\partial F}{\partial v}(x, v)=\frac{d \xi}{d t}}_{\text {since } \xi=L_{F_{x}}(v)}=\underbrace{-\frac{\partial H}{\partial x}(x, \xi)=\frac{\partial F}{\partial x}(x, v)}_{\text {by the claim }} \Longleftrightarrow \quad \text { E-L. }
$$




\section{Homework 15: Legendre Transform}

This set of problems is adapted from [54].

1. Let $f: \mathbb{R} \rightarrow \mathbb{R}$ be a smooth function. $f$ is called strictly convex if $f^{\prime \prime}(x)>0$ for all $x \in \mathbb{R}$. Assuming that $f$ is strictly convex, prove that the following four conditions are equivalent:

(a) $f^{\prime}(x)=0$ for some point $x_{0}$,

(b) $f$ has a local minimum at some point $x_{0}$,

(c) $f$ has a unique (global) minimum at some point $x_{0}$,

(d) $f(x) \rightarrow+\infty$ as $x \rightarrow \pm \infty$.

The function $f$ is stable if it satisfies one (and hence all) of these conditions. For what values of $a$ is the function $e^{x}+a x$ stable? For those values of $a$ for which it is not stable, what does the graph look like?

2. Let $V$ be an $n$-dimensional vector space and $F: V \rightarrow \mathbb{R}$ a smooth function. The function $F$ is said to be strictly convex if for every pair of elements $p, v \in V, v \neq 0$, the restriction of $F$ to the line $\{p+x v \mid x \in \mathbb{R}\}$ is strictly convex.

The hessian of $F$ at $p$ is the quadratic form

$$
d^{2} F_{p}:\left.v \longmapsto \frac{d^{2}}{d x^{2}} F(p+x v)\right|_{x=0} .
$$

Show that $F$ is strictly convex if and only if $d^{2} F_{p}$ is positive definite for all $p \in V$.

Prove the $n$-dimensional analogue of the result you proved in (1). Namely, assuming that $F$ is strictly convex, show that the four following assertions are equivalent:

(a) $d F_{p}=0$ at some point $p_{0}$,

(b) $F$ has a local minimum at some point $p_{0}$,

(c) $F$ has a unique (global) minimum at some point $p_{0}$,

(d) $F(p) \rightarrow+\infty$ as $p \rightarrow \infty$.

3. As in exercise 2 , let $V$ be an $n$-dimensional vector space and $F: V \rightarrow \mathbb{R}$ a smooth function. Since $V$ is a vector space, there is a canonical identification $T_{p}^{*} V \simeq V^{*}$, for every $p \in V$. Therefore, we can define a map

$$
L_{F}: V \longrightarrow V^{*} \quad \text { (Legendre transform) }
$$

by setting

$$
L_{F}(p)=d F_{p} \in T_{p}^{*} V \simeq V^{*} .
$$

Show that, if $F$ is strictly convex, then, for every point $p \in V, L_{F}$ maps a neighborhood of $p$ diffeomorphically onto a neighborhood of $L_{F}(p)$. 
4. A strictly convex function $F: V \rightarrow \mathbb{R}$ is stable if it satisfies the four equivalent conditions of exercise 2 . Given any strictly convex function $F$, we will denote by $S_{F}$ the set of $l \in V^{*}$ for which the function $F_{l}: V \rightarrow \mathbb{R}, p \mapsto F(p)-l(p)$, is stable. Prove that:

(a) The set $S_{F}$ is open and convex.

(b) $L_{F}$ maps $V$ diffeomorphically onto $S_{F}$.

(c) If $\ell \in S_{F}$ and $p_{0}=L_{F}^{-1}(\ell)$, then $p_{0}$ is the unique minimum point of the function $F_{\ell}$.

Let $F^{*}: S_{F} \rightarrow \mathbb{R}$ be the function whose value at $l$ is the quantity $-\min _{p \in V} F_{l}(p)$. Show that $F^{*}$ is a smooth function.

The function $F^{*}$ is called the dual of the function $F$.

5. Let $F$ be a strictly convex function. $F$ is said to have quadratic growth at infinity if there exists a positive-definite quadratic form $Q$ on $V$ and a constant $K$ such that $F(p) \geq Q(p)-K$, for all $p$. Show that, if $F$ has quadratic growth at infinity, then $S_{F}=V^{*}$ and hence $L_{F}$ maps $V$ diffeomorphically onto $V^{*}$.

6. Let $F: V \rightarrow \mathbb{R}$ be strictly convex and let $F^{*}: S_{F} \rightarrow \mathbb{R}$ be the dual function. Prove that for all $p \in V$ and all $\ell \in S_{F}$,

$$
F(p)+F^{*}(\ell) \geq \ell(p) \quad \text { (Young inequality). }
$$

7. On one hand we have $V \times V^{*} \simeq T^{*} V$, and on the other hand, since $V=V^{* *}$, we have $V \times V^{*} \simeq V^{*} \times V \simeq T^{*} V^{*}$.

Let $\alpha_{1}$ be the canonical 1-form on $T^{*} V$ and $\alpha_{2}$ be the canonical 1-form on $T^{*} V^{*}$. Via the identifications above, we can think of both of these forms as living on $V \times V^{*}$. Show that $\alpha_{1}=d \beta-\alpha_{2}$, where $\beta: V \times V^{*} \rightarrow \mathbb{R}$ is the function $\beta(p, \ell)=\ell(p)$.

Conclude that the forms $\omega_{1}=d \alpha_{1}$ and $\omega_{2}=d \alpha_{2}$ satisfy $\omega_{1}=-\omega_{2}$.

8. Let $F: V \rightarrow \mathbb{R}$ be strictly convex. Assume that $F$ has quadratic growth at infinity so that $S_{F}=V^{*}$. Let $\Lambda_{F}$ be the graph of the Legendre transform $L_{F}$. The graph $\Lambda_{F}$ is a lagrangian submanifold of $V \times V^{*}$ with respect to the symplectic form $\omega_{1}$; why? Hence, $\Lambda_{F}$ is also lagrangian for $\omega_{2}$.

Let $\operatorname{pr}_{1}: \Lambda_{F} \rightarrow V$ and $\operatorname{pr}_{2}: \Lambda_{F} \rightarrow V^{*}$ be the restrictions of the projection maps $V \times V^{*} \rightarrow V$ and $V \times V^{*} \rightarrow V^{*}$, and let $i: \Lambda_{F} \hookrightarrow V \times V^{*}$ be the inclusion map. Show that

$$
i^{*} \alpha_{1}=d\left(\mathrm{pr}_{1}\right)^{*} F
$$

Conclude that

$$
i^{*} \alpha_{2}=d\left(i^{*} \beta-\left(\mathrm{pr}_{1}\right)^{*} F\right)=d\left(\mathrm{pr}_{2}\right)^{*} F^{*},
$$

and from this conclude that the inverse of the Legendre transform associated with $F$ is the Legendre transform associated with $F^{*}$. 


\section{Part VIII}

\section{Moment Maps}

The concept of a moment map ${ }^{12}$ is a generalization of that of a hamiltonian function. The notion of a moment map associated to a group action on a symplectic manifold formalizes the Noether principle, which states that to every symmetry (such as a group action) in a mechanical system, there corresponds a conserved quantity.

\section{Actions}

\subsection{One-Parameter Groups of Diffeomorphisms}

Let $M$ be a manifold and $X$ a complete vector field on $M$. Let $\rho_{t}: M \rightarrow M$, $t \in \mathbb{R}$, be the family of diffeomorphisms generated by $X$. For each $p \in M, \rho_{t}(p)$, $t \in \mathbb{R}$, is by definition the unique integral curve of $X$ passing through $p$ at time 0 , i.e., $\rho_{t}(p)$ satisfies

$$
\left\{\begin{aligned}
\rho_{0}(p) & =p \\
\frac{d \rho_{t}(p)}{d t} & =X\left(\rho_{t}(p)\right) .
\end{aligned}\right.
$$

Claim. We have that $\rho_{t} \circ \rho_{s}=\rho_{t+s}$.

Proof. Let $\rho_{s}(q)=p$. We need to show that $\left(\rho_{t} \circ \rho_{s}\right)(q)=\rho_{t+s}(q)$, for all $t \in \mathbb{R}$. Reparametrize as $\tilde{\rho}_{t}(q):=\rho_{t+s}(q)$. Then

$$
\left\{\begin{aligned}
\tilde{\rho}_{0}(q) & =\rho_{s}(q)=p \\
\frac{d \tilde{\rho}_{t}(q)}{d t} & =\frac{d \rho_{t+s}(q)}{d t}=X\left(\rho_{t+s}(q)\right)=X\left(\tilde{\rho}_{t}(q)\right)
\end{aligned}\right.
$$

i.e., $\tilde{\rho}_{t}(q)$ is an integral curve of $X$ through $p$. By uniqueness we must have $\tilde{\rho}_{t}(q)=\rho_{t}(p)$, that is, $\rho_{t+s}(q)=\rho_{t}\left(\rho_{s}(q)\right)$.

Consequence. We have that $\rho_{t}^{-1}=\rho_{-t}$.

In terms of the group $(\mathbb{R},+)$ and the group $(\operatorname{Diff}(M), \circ)$ of all diffeomorphisms of $M$, these results can be summarized as:

Corollary 21.1 The map $\mathbb{R} \rightarrow \operatorname{Diff}(M), t \mapsto \rho_{t}$, is a group homomorphism.

The family $\left\{\rho_{t} \mid t \in \mathbb{R}\right\}$ is then called a one-parameter group of diffeomorphisms of $M$ and denoted

$$
\rho_{t}=\exp t X .
$$

\footnotetext{
${ }^{12}$ Souriau [95] invented the french name "application moment." In the US, East and West coasts could be distinguished by the choice of translation: moment map and momentum map, respectively. We will stick to the more economical version.
} 


\subsection{Lie Groups}

Definition 21.2 $A$ Lie group is a manifold $G$ equipped with a group structure where the group operations

$$
\begin{aligned}
& G \times G \longrightarrow G \quad \text { and } \quad G \longrightarrow G \\
& (a, b) \longmapsto a \cdot b \quad a \longmapsto a^{-1}
\end{aligned}
$$

are smooth maps.

\section{Examples.}

- $\mathbb{R}$ (with addition ${ }^{13}$ ).

- $S^{1}$ regarded as unit complex numbers with multiplication, represents rotations of the plane: $S^{1}=\mathrm{U}(1)=\mathrm{SO}(2)$.

- $\mathrm{U}(n)$, unitary linear transformations of $\mathbb{C}^{n}$.

- $\mathrm{SU}(n)$, unitary linear transformations of $\mathbb{C}^{n}$ with det $=1$.

- $\mathrm{O}(n)$, orthogonal linear transformations of $\mathbb{R}^{n}$.

- $\mathrm{SO}(n)$, elements of $\mathrm{O}(n)$ with $\operatorname{det}=1$.

- $\mathrm{GL}(V)$, invertible linear transformations of a vector space $V$.

Definition 21.3 A representation of a Lie group $G$ on a vector space $V$ is a group homomorphism $G \rightarrow \mathrm{GL}(V)$.

\subsection{Smooth Actions}

Let $M$ be a manifold.

Definition 21.4 An action of a Lie group $G$ on $M$ is a group homomorphism

$$
\begin{aligned}
\psi: G & \longrightarrow \operatorname{Diff}(M) \\
g & \longmapsto \psi_{g} .
\end{aligned}
$$

(We will only consider left actions where $\psi$ is a homomorphism. A right action is defined with $\psi$ being an anti-homomorphism.) The evaluation map associated with an action $\psi: G \rightarrow \operatorname{Diff}(M)$ is

$$
\begin{aligned}
\mathrm{ev}_{\psi}: M \times G & \longrightarrow M \\
(p, g) & \longmapsto \psi_{g}(p) .
\end{aligned}
$$

The action $\psi$ is smooth if $\mathrm{ev}_{\psi}$ is a smooth map.

\footnotetext{
${ }^{13}$ The operation will be omitted when it is clear from the context.
} 
Example. If $X$ is a complete vector field on $M$, then

$$
\begin{aligned}
\rho: \mathbb{R} & \longrightarrow \operatorname{Diff}(M) \\
t & \longmapsto \rho_{t}=\exp t X
\end{aligned}
$$

is a smooth action of $\mathbb{R}$ on $M$.

Every complete vector field gives rise to a smooth action of $\mathbb{R}$ on $M$. Conversely, every smooth action of $\mathbb{R}$ on $M$ is defined by a complete vector field.

$$
\begin{aligned}
&\{\text { complete vector fields on } M\} \stackrel{1-1}{\longleftrightarrow}\{\text { smooth actions of } \mathbb{R} \text { on } M\} \\
& X \longmapsto \exp t X \\
& X_{p}=\left.\frac{d \psi_{t}(p)}{d t}\right|_{t=0} \longleftarrow \psi
\end{aligned}
$$

\subsection{Symplectic and Hamiltonian Actions}

Let $(M, \omega)$ be a symplectic manifold, and $G$ a Lie group. Let $\psi: G \longrightarrow \operatorname{Diff}(M)$ be a (smooth) action.

Definition 21.5 The action $\psi$ is a symplectic action if

$$
\psi: G \longrightarrow \operatorname{Sympl}(M, \omega) \subset \operatorname{Diff}(M),
$$

i.e., $G$ "acts by symplectomorphisms."

\{complete symplectic vector fields on $M\} \stackrel{1-1}{\longleftrightarrow}\{$ symplectic actions of $\mathbb{R}$ on $M$ \}

Example. On $\mathbb{R}^{2 n}$ with $\omega=\sum d x_{i} \wedge d y_{i}$, let $X=-\frac{\partial}{\partial y_{1}}$. The orbits of the action generated by $X$ are lines parallel to the $y_{1}$-axis,

$$
\left\{\left(x_{1}, y_{1}-t, x_{2}, y_{2}, \ldots, x_{n}, y_{n}\right) \mid t \in \mathbb{R}\right\} .
$$

Since $X=X_{x_{1}}$ is hamiltonian (with hamiltonian function $H=x_{1}$ ), this is actually an example of a hamiltonian action of $\mathbb{R}$.

Example. On $S^{2}$ with $\omega=d \theta \wedge d h$ (cylindrical coordinates), let $X=\frac{\partial}{\partial \theta}$. Each orbit is a horizontal circle (called a "parallel") $\{(\theta+t, h) \mid t \in \mathbb{R}\}$. Notice that all orbits of this $\mathbb{R}$-action close up after time $2 \pi$, so that this is an action of $S^{1}$ :

$$
\begin{aligned}
\psi: S^{1} & \longrightarrow \operatorname{Sympl}\left(S^{2}, \omega\right) \\
t & \longmapsto \text { rotation by angle } t \text { around } h \text {-axis } .
\end{aligned}
$$

Since $X=X_{h}$ is hamiltonian (with hamiltonian function $H=h$ ), this is an example of a hamiltonian action of $S^{1}$. 
Definition 21.6 A symplectic action $\psi$ of $S^{1}$ or $\mathbb{R}$ on $(M, \omega)$ is hamiltonian if the vector field generated by $\psi$ is hamiltonian. Equivalently, an action $\psi$ of $S^{1}$ or $\mathbb{R}$ on $(M, \omega)$ is hamiltonian if there is $H: M \rightarrow \mathbb{R}$ with $d H=\imath_{X} \omega$, where $X$ is the vector field generated by $\psi$.

What is a "hamiltonian action" of an arbitrary Lie group?

For the case where $G=\mathbb{T}^{n}=S^{1} \times \ldots \times S^{1}$ is an $n$-torus, an action $\psi: G \rightarrow$ $\operatorname{Sympl}(M, \omega)$ should be called hamiltonian when each restriction

$$
\psi^{i}:=\left.\psi\right|_{i \text { th } S^{1} \text { factor }}: S^{1} \longrightarrow \operatorname{Sympl}(M, \omega)
$$

is hamiltonian in the previous sense with hamiltonian function preserved by the action of the rest of $G$.

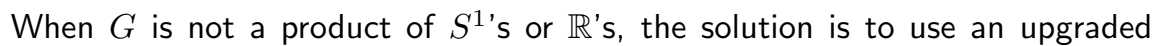
hamiltonian function, known as a moment map. Before its definition though (in Lecture 22), we need a little Lie theory.

\subsection{Adjoint and Coadjoint Representations}

Let $G$ be a Lie group. Given $g \in G$ let

$$
\begin{aligned}
L_{g}: \quad G & \longrightarrow G \\
a & \longmapsto g \cdot a
\end{aligned}
$$

be left multiplication by $g$. A vector field $X$ on $G$ is called left-invariant if $\left(L_{g}\right)_{*} X=X$ for every $g \in G$. (There are similar right notions.)

Let $\mathfrak{g}$ be the vector space of all left-invariant vector fields on $G$. Together with the Lie bracket $[\cdot, \cdot]$ of vector fields, $\mathfrak{g}$ forms a Lie algebra, called the Lie algebra of the Lie group $G$.

Exercise. Show that the map

$$
\begin{aligned}
\mathfrak{g} & \longrightarrow T_{e} G \\
X & \longmapsto X_{e}
\end{aligned}
$$

where $e$ is the identity element in $G$, is an isomorphism of vector spaces.

Any Lie group $G$ acts on itself by conjugation:

$$
\begin{aligned}
G & \longrightarrow \operatorname{Diff}(G) \\
g & \longmapsto \psi_{g},
\end{aligned} \quad \psi_{g}(a)=g \cdot a \cdot g^{-1} .
$$

The derivative at the identity of

$$
\begin{aligned}
\psi_{g}: G & \longrightarrow G \\
a & \longmapsto g \cdot a \cdot g^{-1}
\end{aligned}
$$


is an invertible linear map $\operatorname{Ad}_{g}: \mathfrak{g} \longrightarrow \mathfrak{g}$. Here we identify the Lie algebra $\mathfrak{g}$ with the tangent space $T_{e} G$. Letting $g$ vary, we obtain the adjoint representation (or adjoint action) of $G$ on $\mathfrak{g}$ :

$$
\text { Ad : } \begin{aligned}
G & \longrightarrow \mathrm{GL}(\mathfrak{g}) \\
g & \longmapsto \operatorname{Ad}_{g} .
\end{aligned}
$$

Exercise. Check for matrix groups that

$$
\left.\frac{d}{d t} \operatorname{Ad}_{\exp t X} Y\right|_{t=0}=[X, Y], \quad \forall X, Y \in \mathfrak{g} .
$$

Hint: For a matrix group $G$ (i.e., a subgroup of $\mathrm{GL}(n ; \mathbb{R})$ for some $n$ ), we have

$$
\operatorname{Ad}_{g}(Y)=g Y g^{-1}, \quad \forall g \in G, \forall Y \in \mathfrak{g}
$$

and

$$
[X, Y]=X Y-Y X, \quad \forall X, Y \in \mathfrak{g} .
$$

Let $\langle\cdot, \cdot\rangle$ be the natural pairing between $\mathfrak{g}^{*}$ and $\mathfrak{g}$ :

$$
\begin{aligned}
\langle\cdot, \cdot\rangle: \mathfrak{g}^{*} \times \mathfrak{g} & \longrightarrow \mathbb{R} \\
(\xi, X) & \longmapsto\langle\xi, X\rangle=\xi(X) .
\end{aligned}
$$

Given $\xi \in \mathfrak{g}^{*}$, we define $\operatorname{Ad}_{g}^{*} \xi$ by

$$
\left\langle\operatorname{Ad}_{g}^{*} \xi, X\right\rangle=\left\langle\xi, \operatorname{Ad}_{g^{-1}} X\right\rangle, \quad \text { for any } X \in \mathfrak{g} .
$$

The collection of maps $\operatorname{Ad}_{g}^{*}$ forms the coadjoint representation (or coadjoint action) of $G$ on $\mathfrak{g}^{*}$ :

$$
\begin{aligned}
\operatorname{Ad}^{*}: G & \longrightarrow \operatorname{GL}\left(\mathfrak{g}^{*}\right) \\
g & \longmapsto \operatorname{Ad}_{g}^{*} .
\end{aligned}
$$

We take $g^{-1}$ in the definition of $\operatorname{Ad}_{g}^{*} \xi$ in order to obtain a (left) representation, i.e., a group homomorphism, instead of a "right" representation, i.e., a group antihomomorphism.

Exercise. Show that $\operatorname{Ad}_{g} \circ \operatorname{Ad}_{h}=\operatorname{Ad}_{g h} \quad$ and $\quad \operatorname{Ad}_{g}^{*} \circ \operatorname{Ad}_{h}^{*}=\operatorname{Ad}_{g h}^{*} \cdot \diamond$ 


\section{Homework 16: Hermitian Matrices}

Let $\mathcal{H}$ be the vector space of $n \times n$ complex hermitian matrices.

The unitary group $\mathrm{U}(n)$ acts on $\mathcal{H}$ by conjugation: $A \cdot \xi=A \xi A^{-1}$, for $A \in$ $\mathrm{U}(n), \xi \in \mathcal{H}$.

For each $\lambda=\left(\lambda_{1}, \ldots, \lambda_{n}\right) \in \mathbb{R}^{n}$, let $\mathcal{H}_{\lambda}$ be the set of all $n \times n$ complex hermitian matrices whose spectrum is $\lambda$.

1. Show that the orbits of the $\mathrm{U}(n)$-action are the manifolds $\mathcal{H}_{\lambda}$.

For a fixed $\lambda \in \mathbb{R}^{n}$, what is the stabilizer of a point in $\mathcal{H}_{\lambda}$ ?

Hint: If $\lambda_{1}, \ldots, \lambda_{n}$ are all distinct, the stabilizer of the diagonal matrix is the torus $\mathbb{T}^{n}$ of all diagonal unitary matrices.

2. Show that the symmetric bilinear form on $\mathcal{H}, \quad(X, Y) \mapsto$ trace $(X Y)$, is nondegenerate.

For $\xi \in \mathcal{H}$, define a skew-symmetric bilinear form $\omega_{\xi}$ on $\mathfrak{u}(n)=T_{1} \mathrm{U}(n)=i \mathcal{H}$ (space of skew-hermitian matrices) by

$$
\omega_{\xi}(X, Y)=i \operatorname{trace}([X, Y] \xi), \quad X, Y \in i \mathcal{H} .
$$

Check that $\omega_{\xi}(X, Y)=i$ trace $(X(Y \xi-\xi Y))$ and $Y \xi-\xi Y \in \mathcal{H}$.

Show that the kernel of $\omega_{\xi}$ is $K_{\xi}:=\{Y \in \mathfrak{u}(n) \mid[Y, \xi]=0\}$.

3. Show that $K_{\xi}$ is the Lie algebra of the stabilizer of $\xi$.

Hint: Differentiate the relation $A \xi A^{-1}=\xi$.

Show that the $\omega_{\xi}$ 's induce nondegenerate 2 -forms on the orbits $\mathcal{H}_{\lambda}$. Show that these 2 -forms are closed.

Conclude that all the orbits $\mathcal{H}_{\lambda}$ are compact symplectic manifolds.

4. Describe the manifolds $\mathcal{H}_{\lambda}$.

When all eigenvalues are equal, there is only one point in the orbit.

Suppose that $\lambda_{1} \neq \lambda_{2}=\ldots=\lambda_{n}$. Then the eigenspace associated with $\lambda_{1}$ is a line, and the one associated with $\lambda_{2}$ is the orthogonal hyperplane. Show that there is a diffeomorphism $\mathcal{H}_{\lambda} \simeq \mathbb{C P}^{n-1}$. We have thus exhibited a lot of symplectic forms on $\mathbb{C P}^{n-1}$, on for each pair of distinct real numbers.

What about the other cases?

Hint: When the eigenvalues $\lambda_{1}<\ldots<\lambda_{n}$ are all distinct, any element in $\mathcal{H}_{\lambda}$ defines a family of pairwise orthogonal lines in $\mathbb{C}^{n}$ : its eigenspaces.

5. Show that, for any skew-hermitian matrix $X \in \mathfrak{u}(n)$, the vector field on $\mathcal{H}$ generated by $X \in \mathfrak{u}(n)$ for the $\mathrm{U}(n)$-action by conjugation is $X_{\xi}^{\#}=[X, \xi]$. 


\section{Hamiltonian Actions}

\subsection{Moment and Comoment Maps}

Let

$$
\begin{aligned}
(M, \omega) & \text { be a symplectic manifold, } \\
G & \text { a Lie group, and } \\
\psi: G \rightarrow \operatorname{Sympl}(M, \omega) & \text { a (smooth) symplectic action, i.e., a group homomorphism } \\
& \text { such that the evaluation map } \operatorname{ev}_{\psi}(g, p):=\psi_{g}(p) \text { is smooth. }
\end{aligned}
$$

Case $G=\mathbb{R}$ :

We have the following bijective correspondence:

\{symplectic actions of $\mathbb{R}$ on $M\} \stackrel{1-1}{\longleftrightarrow}$ \{complete symplectic vector fields on $M$ \}

$$
\begin{array}{rll}
\psi & \longmapsto & X_{p}=\frac{d \psi_{t}(p)}{d t} \\
\psi=\exp t X & \longleftarrow & X \\
\text { "flow of } X " & & \text { "vector field generated by } \psi \text { " }
\end{array}
$$

The action $\psi$ is hamiltonian if there exists a function $H: M \rightarrow \mathbb{R}$ such that $d H=\imath_{X} \omega$ where $X$ is the vector field on $M$ generated by $\psi$.

Case $G=S^{1}$ :

An action of $S^{1}$ is an action of $\mathbb{R}$ which is $2 \pi$-periodic: $\psi_{2 \pi}=\psi_{0}$. The $S^{1}$-action is called hamiltonian if the underlying $\mathbb{R}$-action is hamiltonian.

\section{General case:}

Let

$$
\begin{aligned}
(M, \omega) & \text { be a symplectic manifold, } \\
G & \text { a Lie group, } \\
\mathfrak{g} & \text { the Lie algebra of } G, \\
\mathfrak{g}^{*} & \text { the dual vector space of } \mathfrak{g} \text {, and } \\
\psi: G \longrightarrow \operatorname{Sympl}(M, \omega) & \text { a symplectic action. }
\end{aligned}
$$

Definition 22.1 The action $\psi$ is a hamiltonian action if there exists a map

$$
\mu: M \longrightarrow \mathfrak{g}^{*}
$$

satisfying:

1. For each $X \in \mathfrak{g}$, let 
- $\mu^{X}: M \rightarrow \mathbb{R}, \mu^{X}(p):=\langle\mu(p), X\rangle$, be the component of $\mu$ along $X$,

- $X^{\#}$ be the vector field on $M$ generated by the one-parameter subgroup $\{\exp t X \mid t \in \mathbb{R}\} \subseteq G$.

Then

$$
d \mu^{X}=\imath_{X \# \omega}
$$

i.e., $\mu^{X}$ is a hamiltonian function for the vector field $X^{\#}$.

2. $\mu$ is equivariant with respect to the given action $\psi$ of $G$ on $M$ and the coadjoint action $\mathrm{Ad}^{*}$ of $G$ on $\mathfrak{g}^{*}$ :

$$
\mu \circ \psi_{g}=\operatorname{Ad}_{g}^{*} \circ \mu, \quad \text { for all } g \in G .
$$

The vector $(M, \omega, G, \mu)$ is then called a hamiltonian $G$-space and $\mu$ is a moment map.

For connected Lie groups, hamiltonian actions can be equivalently defined in terms of a comoment map

$$
\mu^{*}: \mathfrak{g} \longrightarrow C^{\infty}(M),
$$

with the two conditions rephrased as:

1. $\mu^{*}(X):=\mu^{X}$ is a hamiltonian function for the vector field $X^{\# \text {, }}$

2. $\mu^{*}$ is a Lie algebra homomorphism:

$$
\mu^{*}[X, Y]=\left\{\mu^{*}(X), \mu^{*}(Y)\right\}
$$

where $\{\cdot, \cdot\}$ is the Poisson bracket on $C^{\infty}(M)$.

These definitions match the previous ones for the cases $G=\mathbb{R}, S^{1}$, torus, where equivariance becomes invariance since the coadjoint action is trivial.

Case $G=S^{1}$ (or $\left.\mathbb{R}\right)$ :

Here $\mathfrak{g} \simeq \mathbb{R}, \mathfrak{g}^{*} \simeq \mathbb{R}$. A moment map $\mu: M \longrightarrow \mathbb{R}$ satisfies:

1. For the generator $X=1$ of $\mathfrak{g}$, we have $\mu^{X}(p)=\mu(p) \cdot 1$, i.e., $\mu^{X}=\mu$, and $X^{\#}$ is the standard vector field on $M$ generated by $S^{1}$. Then $d \mu=\imath_{X} \# \omega$.

2. $\mu$ is invariant: $\mathcal{L}_{X \#} \mu=\imath_{X} \# d \mu=0$.

Case $G=\mathbb{T}^{n}=n$-torus:

Here $\mathfrak{g} \simeq \mathbb{R}^{n}, \mathfrak{g}^{*} \simeq \mathbb{R}^{n}$. A moment map $\mu: M \longrightarrow \mathbb{R}^{n}$ satisfies:

1. For each basis vector $X_{i}$ of $\mathbb{R}^{n}, \mu^{X_{i}}$ is a hamiltonian function for $X_{i}^{\#}$.

2. $\mu$ is invariant. 


\subsection{Orbit Spaces}

Let $\psi: G \rightarrow \operatorname{Diff}(M)$ be any action.

Definition 22.2 The orbit of $G$ through $p \in M$ is $\left\{\psi_{g}(p) \mid g \in G\right\}$.

The stabilizer (or isotropy) of $p \in M$ is the subgroup $G_{p}:=\left\{g \in G \mid \psi_{g}(p)=\right.$ $p\}$.

Exercise. If $q$ is in the orbit of $p$, then $G_{q}$ and $G_{p}$ are conjugate subgroups. $\diamond$

Definition 22.3 We say that the action of $G$ on $M$ is ...

- transitive if there is just one orbit,

- free if all stabilizers are trivial $\{e\}$,

- locally free if all stabilizers are discrete.

Let $\sim$ be the orbit equivalence relation; for $p, q \in M$,

$$
p \sim q \quad \Longleftrightarrow \quad p \text { and } q \text { are on the same orbit. }
$$

The space of orbits $M / \sim=M / G$ is called the orbit space. Let

$$
\begin{aligned}
\pi: M & \longrightarrow M / G \\
p & \longmapsto \text { orbit through } p
\end{aligned}
$$

be the point-orbit projection.

\section{Topology of the orbit space:}

We equip $M / G$ with the weakest topology for which $\pi$ is continuous, i.e., $\mathcal{U} \subseteq$ $M / G$ is open if and only if $\pi^{-1}(\mathcal{U})$ is open in $M$. This is called the quotient topology. This topology can be "bad." For instance:

Example. Let $G=\mathbb{R}$ act on $M=\mathbb{R}$ by

$$
t \longmapsto \psi_{t}=\text { multiplication by } e^{t} \text {. }
$$

There are three orbits $\mathbb{R}^{+}, \mathbb{R}^{-}$and $\{0\}$. The point in the three-point orbit space corresponding to the orbit $\{0\}$ is not open, so the orbit space with the quotient topology is not Hausdorff.

Example. Let $G=\mathbb{C} \backslash\{0\}$ act on $M=\mathbb{C}^{n}$ by

$$
\lambda \longmapsto \psi_{\lambda}=\text { multiplication by } \lambda \text {. }
$$

The orbits are the punctured complex lines (through non-zero vectors $z \in \mathbb{C}^{n}$ ), plus one "unstable" orbit through 0 , which has a single point. The orbit space is

$$
M / G=\mathbb{C P}^{n-1} \sqcup\{\text { point }\} .
$$


The quotient topology restricts to the usual topology on $\mathbb{C P}^{n-1}$. The only open set containing \{point in the quotient topology is the full space. Again the quotient topology in $M / G$ is not Hausdorff.

However, it suffices to remove 0 from $\mathbb{C}^{n}$ to obtain a Hausdorff orbit space: $\mathbb{C P}^{n-1}$. Then there is also a compact (yet not complex) description of the orbit space by taking only unit vectors:

$$
\mathbb{C P}^{n-1}=\left(\mathbb{C}^{n} \backslash\{0\}\right) /(\mathbb{C} \backslash\{0\})=S^{2 n-1} / S^{1} .
$$

\subsection{Preview of Reduction}

Let $\omega=\frac{i}{2} \sum d z_{i} \wedge d \bar{z}_{i}=\sum d x_{i} \wedge d y_{i}=\sum r_{i} d r_{i} \wedge d \theta_{i}$ be the standard symplectic form on $\mathbb{C}^{n}$. Consider the following $S^{1}$-action on $\left(\mathbb{C}^{n}, \omega\right)$ :

$$
t \in S^{1} \longmapsto \psi_{t}=\text { multiplication by } t \text {. }
$$

The action $\psi$ is hamiltonian with moment map

$$
\begin{aligned}
\mu: \mathbb{C}^{n} & \longrightarrow \mathbb{R} \\
z & \longmapsto-\frac{|z|^{2}}{2}+\text { constant }
\end{aligned}
$$

since

$$
\begin{aligned}
d \mu & =-\frac{1}{2} d\left(\sum r_{i}^{2}\right) \\
X^{\#} & =\frac{\partial}{\partial \theta_{1}}+\frac{\partial}{\partial \theta_{2}}+\ldots+\frac{\partial}{\partial \theta_{n}} \\
\imath_{X \# \omega} & =-\sum r_{i} d r_{i}=-\frac{1}{2} \sum d r_{i}^{2} .
\end{aligned}
$$

If we choose the constant to be $\frac{1}{2}$, then $\mu^{-1}(0)=S^{2 n-1}$ is the unit sphere. The orbit space of the zero level of the moment map is

$$
\mu^{-1}(0) / S^{1}=S^{2 n-1} / S^{1}=\mathbb{C P}^{n-1} .
$$

$\mathbb{C P}^{n-1}$ is thus called a reduced space. Notice also that the image of the moment map is half-space.

These particular observations are related to major theorems:

Under assumptions (explained in Lectures 23-29),

- [Marsden-Weinstein-Meyer] reduced spaces are symplectic manifolds;

- [Atiyah-Guillemin-Sternberg] the image of the moment map is a convex polytope;

- [Delzant] hamiltonian $\mathbb{T}^{n}$-spaces are classified by the image of the moment map. 


\subsection{Classical Examples}

\section{Example.}

Let $G=\mathrm{SO}(3)=\left\{A \in \mathrm{GL}(3 ; \mathbb{R}) \mid A^{t} A=\mathrm{Id}\right.$ and $\left.\operatorname{det} A=1\right\}$. Then $\mathfrak{g}=\left\{A \in \mathfrak{g l}(3 ; \mathbb{R}) \mid A+A^{t}=0\right\}$ is the space of $3 \times 3$ skew-symmetric matrices and can be identified with $\mathbb{R}^{3}$. The Lie bracket on $\mathfrak{g}$ can be identified with the exterior product via

$$
\begin{gathered}
A=\left[\begin{array}{ccc}
0 & -a_{3} & a_{2} \\
a_{3} & 0 & -a_{1} \\
-a_{2} & a_{1} & 0
\end{array}\right] \longmapsto \vec{a}=\left(a_{1}, a_{2}, a_{3}\right) \\
{[A, B]=A B-B A \longmapsto \vec{a} \times \vec{b}}
\end{gathered}
$$

Exercise. Under the identifications $\mathfrak{g}, \mathfrak{g}^{*} \simeq \mathbb{R}^{3}$, the adjoint and coadjoint actions are the usual $\mathrm{SO}(3)$-action on $\mathbb{R}^{3}$ by rotations.

Therefore, the coadjoint orbits are the spheres in $\mathbb{R}^{3}$ centered at the origin. Homework 17 shows that coadjoint orbits are symplectic.

The name "moment map" comes from being the generalization of linear and angular momenta in classical mechanics.

Translation: Consider $\mathbb{R}^{6}$ with coordinates $x_{1}, x_{2}, x_{3}, y_{1}, y_{2}, y_{3}$ and symplectic form $\omega=\sum d x_{i} \wedge d y_{i}$. Let $\mathbb{R}^{3}$ act on $\mathbb{R}^{6}$ by translations:

$$
\begin{aligned}
\vec{a} \in \mathbb{R}^{3} \longmapsto & \psi_{\vec{a}} \in \operatorname{Sympl}\left(\mathbb{R}^{6}, \omega\right) \\
& \psi_{\vec{a}}(\vec{x}, \vec{y})=(\vec{x}+\vec{a}, \vec{y}) .
\end{aligned}
$$

Then $X^{\#}=a_{1} \frac{\partial}{\partial x_{1}}+a_{2} \frac{\partial}{\partial x_{2}}+a_{3} \frac{\partial}{\partial x_{3}}$ for $X=\vec{a}$, and

$$
\mu: \mathbb{R}^{6} \longrightarrow \mathbb{R}^{3}, \quad \mu(\vec{x}, \vec{y})=\vec{y}
$$

is a moment map, with

$$
\mu^{\vec{a}}(\vec{x}, \vec{y})=\langle\mu(\vec{x}, \vec{y}), \vec{a}\rangle=\vec{y} \cdot \vec{a}
$$

Classically, $\vec{y}$ is called the momentum vector corresponding to the position vector $\vec{x}$, and the map $\mu$ is called the linear momentum.

Rotation: The $\mathrm{SO}(3)$-action on $\mathbb{R}^{3}$ by rotations lifts to a symplectic action $\psi$ on the cotangent bundle $\mathbb{R}^{6}$. The infinitesimal version of this action is

$$
\begin{aligned}
\vec{a} \in \mathbb{R}^{3} \longmapsto & d \psi(\vec{a}) \in \chi^{\mathrm{sympl}}\left(\mathbb{R}^{6}\right) \\
& d \psi(\vec{a})(\vec{x}, \vec{y})=(\vec{a} \times \vec{x}, \vec{a} \times \vec{y}) .
\end{aligned}
$$


Then

$$
\mu: \mathbb{R}^{6} \longrightarrow \mathbb{R}^{3}, \quad \mu(\vec{x}, \vec{y})=\vec{x} \times \vec{y}
$$

is a moment map, with

$$
\mu^{\vec{a}}(\vec{x}, \vec{y})=\langle\mu(\vec{x}, \vec{y}), \vec{a}\rangle=(\vec{x} \times \vec{y}) \cdot \vec{a} .
$$

The map $\mu$ is called the angular momentum. 


\section{Homework 17: Coadjoint Orbits}

Let $G$ be a Lie group, $\mathfrak{g}$ its Lie algebra and $\mathfrak{g}^{*}$ the dual vector space of $\mathfrak{g}$.

1. Let ${ }^{\mathfrak{g}} X^{\#}$ be the vector field generated by $X \in \mathfrak{g}$ for the adjoint representation of $G$ on $\mathfrak{g}$. Show that

$$
{ }^{\mathfrak{g}} X_{Y}^{\#}=[X, Y] \quad \forall Y \in \mathfrak{g} .
$$

2. Let $X^{\#}$ be the vector field generated by $X \in \mathfrak{g}$ for the coadjoint representation of $G$ on $\mathfrak{g}^{*}$. Show that

$$
\left\langle X_{\xi}^{\#}, Y\right\rangle=\langle\xi,[Y, X]\rangle \quad \forall Y \in \mathfrak{g} .
$$

3. For any $\xi \in \mathfrak{g}^{*}$, define a skew-symmetric bilinear form on $\mathfrak{g}$ by

$$
\omega_{\xi}(X, Y):=\langle\xi,[X, Y]\rangle \text {. }
$$

Show that the kernel of $\omega_{\xi}$ is the Lie algebra $\mathfrak{g}_{\xi}$ of the stabilizer of $\xi$ for the coadjoint representation.

4. Show that $\omega_{\xi}$ defines a nondegenerate 2 -form on the tangent space at $\xi$ to the coadjoint orbit through $\xi$.

5. Show that $\omega_{\xi}$ defines a closed 2-form on the orbit of $\xi$ in $\mathfrak{g}^{*}$.

Hint: The tangent space to the orbit being generated by the vector fields $X^{\#}$, this is a consequence of the Jacobi identity in $\mathfrak{g}$.

This canonical symplectic form on the coadjoint orbits in $\mathfrak{g}^{*}$ is also known as the Lie-Poisson or Kostant-Kirillov symplectic structure.

6. The Lie algebra structure of $\mathfrak{g}$ defines a canonical Poisson structure on $\mathfrak{g}^{*}$ :

$$
\{f, g\}(\xi):=\left\langle\xi,\left[d f_{\xi}, d g_{\xi}\right]\right\rangle
$$

for $f, g \in C^{\infty}\left(\mathfrak{g}^{*}\right)$ and $\xi \in \mathfrak{g}^{*}$. Notice that $d f_{\xi}: T_{\xi} \mathfrak{g}^{*} \simeq \mathfrak{g}^{*} \rightarrow \mathbb{R}$ is identified with an element of $\mathfrak{g} \simeq \mathfrak{g}^{* *}$.

Check that $\{\cdot, \cdot\}$ satisfies the Leibniz rule:

$$
\{f, g h\}=g\{f, h\}+h\{f, g\} .
$$

7. Show that the jacobiator

$$
J(f, g, h):=\{\{f, g\}, h\}+\{\{g, h\}, f\}+\{\{h, f\}, g\}
$$

is a trivector field, i.e., $J$ is a skew-symmetric trilinear map $C^{\infty}\left(\mathfrak{g}^{*}\right) \times C^{\infty}\left(\mathfrak{g}^{*}\right) \times$ $C^{\infty}\left(\mathfrak{g}^{*}\right) \rightarrow C^{\infty}\left(\mathfrak{g}^{*}\right)$, which is a derivation in each argument.

Hint: Being a derivation amounts to the Leibniz rule from exercise 6.

8. Show that $J \equiv 0$, i.e., $\{\cdot, \cdot\}$ satisfies the Jacobi identity.

Hint: Follows from the Jacobi identity for $[\cdot, \cdot]$ in $\mathfrak{g}$. It is enough to check on coordinate functions. 



\section{Part IX}

\section{Symplectic Reduction}

The phase space of a system of $n$ particles is the space parametrizing the position and momenta of the particles. The mathematical model for the phase space is a symplectic manifold. Classical physicists realized that, whenever there is a symmetry group of dimension $k$ acting on a mechanical system, then the number of degrees of freedom for the position and momenta of the particles may be reduced by $2 k$. Symplectic reduction formulates this feature mathematically.

\section{The Marsden-Weinstein-Meyer Theorem}

\subsection{Statement}

Theorem 23.1 (Marsden-Weinstein-Meyer [77, 85]) Let $(M, \omega, G, \mu)$ be a hamiltonian $G$-space for a compact Lie group $G$. Let $i: \mu^{-1}(0) \hookrightarrow M$ be the inclusion map. Assume that $G$ acts freely on $\mu^{-1}(0)$. Then

- the orbit space $M_{\mathrm{red}}=\mu^{-1}(0) / G$ is a manifold,

- $\pi: \mu^{-1}(0) \rightarrow M_{\text {red }}$ is a principal G-bundle, and

- there is a symplectic form $\omega_{\text {red }}$ on $M_{\text {red }}$ satisfying $i^{*} \omega=\pi^{*} \omega_{\text {red }}$.

Definition 23.2 The pair $\left(M_{\text {red }}, \omega_{\text {red }}\right)$ is called the reduction of $(M, \omega)$ with respect to $G, \mu$, or the reduced space, or the symplectic quotient, or the MarsdenWeinstein-Meyer quotient, etc.

Low-brow proof for the case $G=S^{1}$ and $\operatorname{dim} M=4$.

In this case the moment map is $\mu: M \rightarrow \mathbb{R}$. Let $p \in \mu^{-1}(0)$. Choose local coordinates:

- $\theta$ along the orbit through $p$,

- $\mu$ given by the moment map, and

- $\eta_{1}, \eta_{2}$ pullback of coordinates on $\mu^{-1}(0) / S^{1}$.

Then the symplectic form can be written

$$
\omega=A d \theta \wedge d \mu+B_{j} d \theta \wedge d \eta_{j}+C_{j} d \mu \wedge d \eta_{j}+D d \eta_{1} \wedge d \eta_{2} .
$$

Since $d \mu=\imath\left(\frac{\partial}{\partial \theta}\right) \omega$, we must have $A=1, B_{j}=0$. Hence,

$$
\omega=d \theta \wedge d \mu+C_{j} d \mu \wedge d \eta_{j}+D d \eta_{1} \wedge d \eta_{2} .
$$

Since $\omega$ is symplectic, we must have $D \neq 0$. Therefore, $i^{*} \omega=D d \eta_{1} \wedge d \eta_{2}$ is the pullback of a symplectic form on $M_{\text {red }}$.

The actual proof of the Marsden-Weinstein-Meyer theorem requires the following ingredients. 


\subsection{Ingredients}

1. Let $\mathfrak{g}_{p}$ be the Lie algebra of the stabilizer of $p \in M$. Then $d \mu_{p}: T_{p} M \rightarrow \mathfrak{g}^{*}$ has

$$
\begin{aligned}
\operatorname{ker} d \mu_{p} & =\left(T_{p} \mathcal{O}_{p}\right)^{\omega_{p}} \\
\operatorname{im} d \mu_{p} & =\mathfrak{g}_{p}^{0}
\end{aligned}
$$

where $\mathcal{O}_{p}$ is the $G$-orbit through $p$, and $\mathfrak{g}_{p}^{0}=\left\{\xi \in \mathfrak{g}^{*} \mid\langle\xi, X\rangle=0, \forall X \in \mathfrak{g}_{p}\right\}$ is the annihilator of $\mathfrak{g}_{p}$.

Proof. Stare at the expression $\omega_{p}\left(X_{p}^{\#}, v\right)=\left\langle d \mu_{p}(v), X\right\rangle$, for all $v \in T_{p} M$ and all $X \in \mathfrak{g}$, and count dimensions.

\section{Consequences:}

- The action is locally free at $p$

$\Longleftrightarrow \mathfrak{g}_{p}=\{0\}$

$\Longleftrightarrow d \mu_{p}$ is surjective

$\Longleftrightarrow p$ is a regular point of $\mu$.

- $G$ acts freely on $\mu^{-1}(0)$

$\Longrightarrow 0$ is a regular value of $\mu$

$\Longrightarrow \mu^{-1}(0)$ is a closed submanifold of $M$ of codimension equal to $\operatorname{dim} G$.

- $G$ acts freely on $\mu^{-1}(0)$

$\Longrightarrow T_{p} \mu^{-1}(0)=\operatorname{ker} d \mu_{p}\left(\right.$ for $\left.p \in \mu^{-1}(0)\right)$

$\Longrightarrow T_{p} \mu^{-1}(0)$ and $T_{p} \mathcal{O}_{p}$ are symplectic orthocomplements in $T_{p} M$.

In particular, the tangent space to the orbit through $p \in \mu^{-1}(0)$ is an isotropic subspace of $T_{p} M$. Hence, orbits in $\mu^{-1}(0)$ are isotropic.

Since any tangent vector to the orbit is the value of a vector field generated by the group, we can confirm that orbits are isotropic directly by computing, for any $X, Y \in \mathfrak{g}$ and any $p \in \mu^{-1}(0)$,

$$
\begin{aligned}
\omega_{p}\left(X_{p}^{\#}, Y_{p}^{\#}\right) & =\text { hamiltonian function for }\left[Y^{\#}, X^{\#}\right] \text { at } p \\
& =\text { hamiltonian function for }[Y, X]^{\#} \text { at } p \\
& =\mu^{[Y, X]}(p)=0 .
\end{aligned}
$$

2. Lemma 23.3 Let $(V, \omega)$ be a symplectic vector space. Suppose that $I$ is an isotropic subspace, that is, $\left.\omega\right|_{I} \equiv 0$. Then $\omega$ induces a canonical symplectic form $\Omega$ on $I^{\omega} / I$.

Proof. Let $u, v \in I^{\omega}$, and $[u],[v] \in I^{\omega} / I$. Define $\Omega([u],[v])=\omega(u, v)$.

- $\Omega$ is well-defined:

$$
\omega(u+i, v+j)=\omega(u, v)+\underbrace{\omega(u, j)}_{0}+\underbrace{\omega(i, v)}_{0}+\underbrace{\omega(i, j)}_{0}, \quad \forall i, j \in I .
$$


- $\Omega$ is nondegenerate:

Suppose that $u \in I^{\omega}$ has $\omega(u, v)=0$, for all $v \in I^{\omega}$.

Then $u \in\left(I^{\omega}\right)^{\omega}=I$, i.e., $[u]=0$.

3. Theorem 23.4 If a compact Lie group $G$ acts freely on a manifold $M$, then $M / G$ is a manifold and the map $\pi: M \rightarrow M / G$ is a principal $G$-bundle.

Proof. We will first show that, for any $p \in M$, the $G$-orbit through $p$ is a compact embedded submanifold of $M$ diffeomorphic to $G$.

Since the action is smooth, the evaluation map ev $: G \times M \rightarrow M, \operatorname{ev}(g, p)=$ $g \cdot p$, is smooth. Let $\mathrm{ev}_{p}: G \rightarrow M$ be defined by $\operatorname{ev}_{p}(g)=g \cdot p$. The map $\mathrm{ev}_{p}$ provides the embedding we seek:

The image of $\mathrm{ev}_{p}$ is the $G$-orbit through $p$. Injectivity of $\mathrm{ev}_{p}$ follows from the action of $G$ being free. The map ev $p$ is proper because, if $A$ is a compact, hence closed, subset of $M$, then its inverse image $\left(\operatorname{ev}_{p}\right)^{-1}(A)$, being a closed subset of the compact Lie group $G$, is also compact. It remains to show that $\mathrm{ev}_{p}$ is an immersion. For $X \in \mathfrak{g} \simeq T_{e} G$, we have

$$
d\left(\operatorname{ev}_{p}\right)_{e}(X)=0 \Longleftrightarrow X_{p}^{\#}=0 \Longleftrightarrow X=0,
$$

as the action is free. We conclude that $d\left(\operatorname{ev}_{p}\right)_{e}$ is injective. At any other point $g \in G$, for $X \in T_{g} G$, we have

$$
d\left(\mathrm{ev}_{p}\right)_{g}(X)=0 \Longleftrightarrow d\left(\mathrm{ev}_{p} \circ R_{g}\right)_{e} \circ\left(d R_{g^{-1}}\right)_{g}(X)=0,
$$

where $R_{g}: G \rightarrow G$ is right multiplication by $g$. But $\mathrm{ev}_{p} \circ R_{g}=\mathrm{ev}_{g \cdot p}$ has an injective differential at $e$, and $\left(d R_{g^{-1}}\right)_{g}$ is an isomorphism. It follows that $d\left(\mathrm{ev}_{p}\right)_{g}$ is always injective.

Exercise. Show that, even if the action is not free, the $G$-orbit through $p$ is a compact embedded submanifold of $M$. In that case, the orbit is diffeomorphic to the quotient of $G$ by the isotropy of $p$ : $\mathcal{O}_{p} \simeq G / G_{p}$.

Let $S$ be a transverse section to $\mathcal{O}_{p}$ at $p$; this is called a slice. Choose a coordinate system $x_{1}, \ldots, x_{n}$ centered at $p$ such that

$$
\begin{array}{lll}
\mathcal{O}_{p} \simeq G & : x_{1}=\ldots=x_{k}=0 \\
S & : x_{k+1}=\ldots=x_{n}=0 .
\end{array}
$$

Let $S_{\varepsilon}=S \cap B_{\varepsilon}\left(0, \mathbb{R}^{n}\right)$ where $B_{\varepsilon}\left(0, \mathbb{R}^{n}\right)$ is the ball of radius $\varepsilon$ centered at 0 in $\mathbb{R}^{n}$. Let $\eta: G \times S \rightarrow M, \eta(g, s)=g \cdot s$. Apply the following equivariant tubular neighborhood theorem. 
Theorem 23.5 (Slice Theorem) Let $G$ be a compact Lie group acting on a manifold $M$ such that $G$ acts freely at $p \in M$. For sufficiently small $\varepsilon, \eta: G \times S_{\varepsilon} \rightarrow M$ maps $G \times S_{\varepsilon}$ diffeomorphically onto a $G$-invariant neighborhood $\mathcal{U}$ of the $G$-orbit through $p$.

The proof of this slice theorem is sketched further below.

Corollary 23.6 If the action of $G$ is free at $p$, then the action is free on $\mathcal{U}$.

Corollary 23.7 The set of points where $G$ acts freely is open.

Corollary 23.8 The set $G \times S_{\varepsilon} \simeq \mathcal{U}$ is $G$-invariant. Hence, the quotient $\mathcal{U} / G \simeq S_{\varepsilon}$ is smooth.

Conclusion of the proof that $M / G$ is a manifold and $\pi: M \rightarrow M / G$ is a smooth fiber map.

For $p \in M$, let $q=\pi(p) \in M / G$. Choose a $G$-invariant neighborhood $\mathcal{U}$ of $p$ as in the slice theorem: $\mathcal{U} \simeq G \times S$ (where $S=S_{\varepsilon}$ for an appropriate $\varepsilon)$. Then $\pi(\mathcal{U})=\mathcal{U} / G=: \mathcal{V}$ is an open neighborhood of $q$ in $M / G$. By the slice theorem, $S \stackrel{\simeq}{\rightarrow} \mathcal{V}$ is a homeomorphism. We will use such neighborhoods $\mathcal{V}$ as charts on $M / G$. To show that the transition functions associated with these charts are smooth, consider two $G$-invariant open sets $\mathcal{U}_{1}, \mathcal{U}_{2}$ in $M$ and corresponding slices $S_{1}, S_{2}$ of the $G$-action. Then $S_{12}=S_{1} \cap \mathcal{U}_{2}, S_{21}=$ $S_{2} \cap \mathcal{U}_{1}$ are both slices for the $G$-action on $\mathcal{U}_{1} \cap \mathcal{U}_{2}$. To compute the transition map $S_{12} \rightarrow S_{21}$, consider the diagram

$$
\begin{aligned}
& S_{12} \stackrel{\simeq}{\longrightarrow} \mathrm{id} \times S_{12} \quad \hookrightarrow \quad G \times S_{12} \\
& \searrow \quad \mathcal{U}_{1} \cap \mathcal{U}_{2} \\
& S_{21} \stackrel{\simeq}{\longrightarrow} \mathrm{id} \times S_{21} \quad \hookrightarrow G \times S_{21}
\end{aligned}
$$

Then the composition

$$
S_{12} \hookrightarrow \mathcal{U}_{1} \cap \mathcal{U}_{2} \stackrel{\simeq}{\longrightarrow} G \times S_{21} \stackrel{p r}{\longrightarrow} S_{21}
$$

is smooth.

Finally, we need to show that $\pi: M \rightarrow M / G$ is a smooth fiber map. For $p \in$ $M, q=\pi(p)$, choose a $G$-invariant neighborhood $\mathcal{U}$ of the $G$-orbit through $p$ of the form $\eta: G \times S \stackrel{\simeq}{\rightarrow} \mathcal{U}$. Then $\mathcal{V}=\mathcal{U} / G \simeq S$ is the corresponding neighborhood of $q$ in $M / G$ :

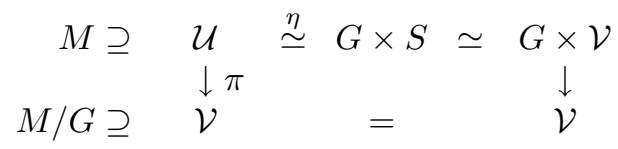


Since the projection on the right is smooth, $\pi$ is smooth.

Exercise. Check that the transition functions for the bundle defined by $\pi$ are smooth.

Sketch for the proof of the slice theorem. We need to show that, for $\varepsilon$ sufficiently small, $\eta: G \times S_{\varepsilon} \rightarrow \mathcal{U}$ is a diffeomorphism where $\mathcal{U} \subseteq M$ is a $G$-invariant neighborhood of the $G$-orbit through $p$. Show that:

(a) $d \eta_{(\mathrm{id}, p)}$ is bijective.

(b) Let $G$ act on $G \times S$ by the product of its left action on $G$ and trivial action on $S$. Then $\eta: G \times S \rightarrow M$ is $G$-equivariant.

(c) $d \eta$ is bijective at all points of $G \times\{p\}$. This follows from (a) and (b).

(d) The set $G \times\{p\}$ is compact, and $\eta: G \times S \rightarrow M$ is injective on $G \times\{p\}$ with $d \eta$ bijective at all these points. By the implicit function theorem, there is a neighborhood $\mathcal{U}_{0}$ of $G \times\{p\}$ in $G \times S$ such that $\eta$ maps $\mathcal{U}_{0}$ diffeomorphically onto a neighborhood $\mathcal{U}$ of the $G$-orbit through $p$.

(e) The sets $G \times S_{\varepsilon}$, varying $\varepsilon$, form a neighborhood base for $G \times\{p\}$ in $G \times S$. So in (d) we may take $\mathcal{U}_{0}=G \times S_{\varepsilon}$.

\subsection{Proof of the Marsden-Weinstein-Meyer Theorem}

Since

$G$ acts freely on $\mu^{-1}(0) \Longrightarrow d \mu_{p}$ is surjective for all $p \in \mu^{-1}(0)$

$\Longrightarrow 0$ is a regular value

$\Longrightarrow \mu^{-1}(0)$ is a submanifold of codimension $=\operatorname{dim} G$

for the first two parts of the Marsden-Weinstein-Meyer theorem it is enough to apply the third ingredient from Section 23.2 to the free action of $G$ on $\mu^{-1}(0)$.

At $p \in \mu^{-1}(0)$ the tangent space to the orbit $T_{p} \mathcal{O}_{p}$ is an isotropic subspace of the symplectic vector space $\left(T_{p} M, \omega_{p}\right)$, i.e., $T_{p} \mathcal{O}_{p} \subseteq\left(T_{p} \mathcal{O}_{p}\right)^{\omega}$.

$$
\left(T_{p} \mathcal{O}_{p}\right)^{\omega}=\operatorname{ker} d \mu_{p}=T_{p} \mu^{-1}(0) .
$$

The lemma (second ingredient) gives a canonical symplectic structure on the quotient $T_{p} \mu^{-1}(0) / T_{p} \mathcal{O}_{p}$. The point $[p] \in M_{\text {red }}=\mu^{-1}(0) / G$ has tangent space $T_{[p]} M_{\text {red }} \simeq T_{p} \mu^{-1}(0) / T_{p} \mathcal{O}_{p}$. Thus the lemma defines a nondegenerate 2 -form $\omega_{\text {red }}$ on $M_{\text {red }}$. This is well-defined because $\omega$ is $G$-invariant.

By construction $i^{*} \omega=\pi^{*} \omega_{\text {red }}$ where

$$
\begin{gathered}
\mu^{-1}(0) \stackrel{i}{\hookrightarrow} \quad M \\
\downarrow \pi \\
M_{\text {red }}
\end{gathered}
$$


Hence, $\pi^{*} d \omega_{\text {red }}=d \pi^{*} \omega_{\text {red }}=d \imath^{*} \omega=\imath^{*} d \omega=0$. The closedness of $\omega_{\text {red }}$ follows from the injectivity of $\pi^{*}$.

Remark. Suppose that another Lie group $H$ acts on $(M, \omega)$ in a hamiltonian way with moment map $\phi: M \rightarrow \mathfrak{h}^{*}$. If the $H$-action commutes with the $G$-action, and if $\phi$ is $G$-invariant, then $M_{\text {red }}$ inherits a hamiltonian action of $H$, with moment map $\phi_{\text {red }}: M_{\text {red }} \rightarrow \mathfrak{h}^{*}$ satisfying $\phi_{\text {red }} \circ \pi=\phi \circ i$. 


\section{Reduction}

\subsection{Noether Principle}

Let $(M, \omega, G, \mu)$ be a hamiltonian $G$-space.

Theorem 24.1 (Noether) A function $f: M \rightarrow \mathbb{R}$ is $G$-invariant if and only if $\mu$ is constant on the trajectories of the hamiltonian vector field of $f$.

Proof. Let $v_{f}$ be the hamiltonian vector field of $f$. Let $X \in \mathfrak{g}$ and $\mu^{X}=\langle\mu, X\rangle$ : $M \rightarrow \mathbb{R}$. We have

$$
\begin{aligned}
\mathcal{L}_{v_{f}} \mu^{X} & =\imath_{v_{f}} d \mu^{X}=\imath_{v_{f}} \imath_{X} \omega \\
& =-\imath_{X^{\#}} \imath_{v_{f}} \omega=-\imath_{X} d f \\
& =-\mathcal{L}_{X^{\#}} f=0
\end{aligned}
$$

because $f$ is $G$-invariant.

Definition 24.2 A G-invariant function $f: M \rightarrow \mathbb{R}$ is called an integral of motion of $(M, \omega, G, \mu)$. If $\mu$ is constant on the trajectories of a hamiltonian vector field $v_{f}$, then the corresponding one-parameter group of diffeomorphisms $\left\{\exp t v_{f} \mid t \in \mathbb{R}\right\}$ is called a symmetry of $(M, \omega, G, \mu)$.

The Noether principle asserts that there is a one-to-one correspondence between symmetries and integrals of motion.

\subsection{Elementary Theory of Reduction}

Finding a symmetry for a $2 n$-dimensional mechanical problem may reduce it to a $(2 n-2)$-dimensional problem as follows: an integral of motion $f$ for a $2 n$ dimensional hamiltonian system $(M, \omega, H)$ may enable us to understand the trajectories of this system in terms of the trajectories of a $(2 n-2)$-dimensional hamiltonian system $\left(M_{\text {red }}, \omega_{\text {red }}, H_{\text {red }}\right)$. To make this precise, we will describe this process locally. Suppose that $\mathcal{U}$ is an open set in $M$ with Darboux coordinates $x_{1}, \ldots, x_{n}, \xi_{1}, \ldots, \xi_{n}$ such that $f=\xi_{n}$ for this chart, and write $H$ in these coordinates: $H=H\left(x_{1}, \ldots, x_{n}, \xi_{1}, \ldots, \xi_{n}\right)$. Then

$$
\xi_{n} \text { is an integral of motion } \Longrightarrow\left\{\begin{array}{l}
\text { the trajectories of } v_{H} \text { lie on the } \\
\text { hyperplane } \xi_{n}=\text { constant } \\
\left\{\begin{array}{l}
\left.\xi_{n}, H\right\}=0=-\frac{\partial H}{\partial x_{n}} \\
\Longrightarrow H=H\left(x_{1}, \ldots, x_{n-1}, \xi_{1}, \ldots, \xi_{n}\right) .
\end{array}\right.
\end{array}\right.
$$


If we set $\xi_{n}=c$, the motion of the system on this hyperplane is described by the following Hamilton equations:

$$
\left\{\begin{array}{cccc}
\frac{d x_{1}}{d t} & = & \frac{\partial H}{\partial \xi_{1}} & \left(x_{1}, \ldots, x_{n-1}, \xi_{1}, \ldots, \xi_{n-1}, c\right) \\
\vdots & & & \\
\frac{d x_{n-1}}{d t} & = & \frac{\partial H}{\partial \xi_{n-1}} & \left(x_{1}, \ldots, x_{n-1}, \xi_{1}, \ldots, \xi_{n-1}, c\right) \\
\frac{d \xi_{1}}{d t} & = & -\frac{\partial H}{\partial x_{1}} & \left(x_{1}, \ldots, x_{n-1}, \xi_{1}, \ldots, \xi_{n-1}, c\right) \\
\vdots & & \\
\frac{d \xi_{n-1}}{d t} & = & -\frac{\partial H}{\partial x_{n-1}} & \left(x_{1}, \ldots, x_{n-1}, \xi_{1}, \ldots, \xi_{n-1}, c\right) \\
\frac{d x_{n}}{d t} & = & \frac{\partial H}{\partial \xi_{n}} \\
\frac{d \xi_{n}}{d t} & = & -\frac{\partial H}{\partial x_{n}}=0 .
\end{array}\right.
$$

The reduced phase space is

$$
\begin{aligned}
\mathcal{U}_{\text {red }}= & \left\{\left(x_{1}, \ldots, x_{n-1}, \xi_{1}, \ldots, \xi_{n-1}\right) \in \mathbb{R}^{2 n-2} \mid\right. \\
& \left.\left(x_{1}, \ldots, x_{n-1}, a, \xi_{1}, \ldots, \xi_{n-1}, c\right) \in \mathcal{U} \text { for some } a\right\} .
\end{aligned}
$$

The reduced hamiltonian is

$$
\begin{aligned}
& H_{\text {red }}: \mathcal{U}_{\text {red }} \longrightarrow \mathbb{R}, \\
& H_{\text {red }}\left(x_{1}, \ldots, x_{n-1}, \xi_{1}, \ldots, \xi_{n-1}\right)=H\left(x_{1}, \ldots, x_{n-1}, \xi_{1}, \ldots, \xi_{n-1}, c\right) .
\end{aligned}
$$

In order to find the trajectories of the original system on the hypersurface $\xi_{n}=c$, we look for the trajectories

$$
x_{1}(t), \ldots, x_{n-1}(t), \xi_{1}(t), \ldots, \xi_{n-1}(t)
$$

of the reduced system on $\mathcal{U}_{\text {red }}$. We integrate the equation

$$
\frac{d x_{n}}{d t}(t)=\frac{\partial H}{\partial \xi_{n}}\left(x_{1}(t), \ldots, x_{n-1}(t), \xi_{1}(t), \ldots, \xi_{n-1}(t), c\right)
$$

to obtain the original trajectories

$$
\left\{\begin{array}{l}
x_{n}(t)=x_{n}(0)+\int_{0}^{t} \frac{\partial H}{\partial \xi_{n}}(\ldots) d t \\
\xi_{n}(t)=c .
\end{array}\right.
$$




\subsection{Reduction for Product Groups}

Let $G_{1}$ and $G_{2}$ be compact connected Lie groups and let $G=G_{1} \times G_{2}$. Then

$$
\mathfrak{g}=\mathfrak{g}_{1} \oplus \mathfrak{g}_{2} \quad \text { and } \quad \mathfrak{g}^{*}=\mathfrak{g}_{1}^{*} \oplus \mathfrak{g}_{2}^{*}
$$

Suppose that $(M, \omega, G, \psi)$ is a hamiltonian $G$-space with moment map

$$
\psi: M \longrightarrow \mathfrak{g}_{1}^{*} \oplus \mathfrak{g}_{2}^{*}
$$

Write $\psi=\left(\psi_{1}, \psi_{2}\right)$ where $\psi_{i}: M \rightarrow \mathfrak{g}_{i}^{*}$ for $i=1,2$. The fact that $\psi$ is equivariant implies that $\psi_{1}$ is invariant under $G_{2}$ and $\psi_{2}$ is invariant under $G_{1}$. Now reduce $(M, \omega)$ with respect to the $G_{1}$-action. Let

$$
Z_{1}=\psi_{1}^{-1}(0)
$$

Assume that $G_{1}$ acts freely on $Z_{1}$. Let $M_{1}=Z_{1} / G_{1}$ be the reduced space and let $\omega_{1}$ be the corresponding reduced symplectic form. The action of $G_{2}$ on $Z_{1}$ commutes with the $G_{1}$-action. Since $G_{2}$ preserves $\omega$, it follows that $G_{2}$ acts symplectically on $\left(M_{1}, \omega_{1}\right)$. Since $G_{1}$ preserves $\psi_{2}, G_{1}$ also preserves $\psi_{2} \circ \iota_{1}: Z_{1} \rightarrow \mathfrak{g}_{2}^{*}$, where $\iota_{1}: Z_{1} \hookrightarrow M$ is inclusion. Thus $\psi_{2} \circ \iota_{1}$ is constant on fibers of $Z_{1} \stackrel{p_{1}}{\rightarrow} M_{1}$. We conclude that there exists a smooth map $\mu_{2}: M_{1} \rightarrow \mathfrak{g}_{2}^{*}$ such that $\mu_{2} \circ p_{1}=\psi_{2} \circ \iota_{1}$.

Exercise. Show that:

(a) the map $\mu_{2}$ is a moment map for the action of $G_{2}$ on $\left(M_{1}, \omega_{1}\right)$, and

(b) if $G$ acts freely on $\psi^{-1}(0,0)$, then $G_{2}$ acts freely on $\mu_{2}^{-1}(0)$, and there is a natural symplectomorphism

$$
\mu_{2}^{-1}(0) / G_{2} \simeq \psi^{-1}(0,0) / G
$$

This technique of performing reduction with respect to one factor of a product group at a time is called reduction in stages. It may be extended to reduction by a normal subgroup $H \subset G$ and by the corresponding quotient group $G / H$.

\subsection{Reduction at Other Levels}

Suppose that a compact Lie group $G$ acts on a symplectic manifold $(M, \omega)$ in a hamiltonian way with moment map $\mu: M \rightarrow \mathfrak{g}^{*}$. Let $\xi \in \mathfrak{g}^{*}$.

To reduce at the level $\xi$ of $\mu$, we need $\mu^{-1}(\xi)$ to be preserved by $G$, or else take the $G$-orbit of $\mu^{-1}(\xi)$, or else take the quotient by the maximal subgroup of $G$ which preserves $\mu^{-1}(\xi)$.

Since $\mu$ is equivariant,

$$
\begin{aligned}
G \text { preserves } \mu^{-1}(\xi) & \Longleftrightarrow G \text { preserves } \xi \\
& \Longleftrightarrow \operatorname{Ad}_{g}^{*} \xi=\xi, \forall g \in G .
\end{aligned}
$$


Of course the level 0 is always preserved. Also, when $G$ is a torus, any level is preserved and reduction at $\xi$ for the moment map $\mu$, is equivalent to reduction at 0 for a shifted moment map $\phi: M \rightarrow \mathfrak{g}^{*}, \phi(p):=\mu(p)-\xi$.

Let $\mathcal{O}$ be a coadjoint orbit in $\mathfrak{g}^{*}$ equipped with the canonical symplectic form (also know as the Kostant-Kirillov symplectic form or the Lie-Poisson symplectic form) $\omega_{\mathcal{O}}$ defined in Homework 17 . Let $\mathcal{O}^{-}$be the orbit $\mathcal{O}$ equipped with $-\omega_{\mathcal{O}}$. The natural product action of $G$ on $M \times \mathcal{O}^{-}$is hamiltonian with moment map $\mu_{\mathcal{O}}(p, \xi)=\mu(p)-\xi$. If the Marsden-Weinstein-Meyer hypothesis is satisfied for $M \times \mathcal{O}^{-}$, then one obtains a reduced space with respect to the coadjoint orbit $\mathcal{O}$.

\subsection{Orbifolds}

Example. Let $G=\mathbb{T}^{n}$ be an $n$-torus. For any $\xi \in\left(\mathfrak{t}^{n}\right)^{*}, \mu^{-1}(\xi)$ is preserved by the $\mathbb{T}^{n}$-action. Suppose that $\xi$ is a regular value of $\mu$. (By Sard's theorem, the singular values of $\mu$ form a set of measure zero.) Then $\mu^{-1}(\xi)$ is a submanifold of codimension $n$. Note that

$$
\begin{aligned}
\xi \text { regular } & \Longrightarrow d \mu_{p} \text { is surjective at all } p \in \mu^{-1}(\xi) \\
& \Longrightarrow \mathfrak{g}_{p}=0 \text { for all } p \in \mu^{-1}(\xi) \\
& \Longrightarrow \text { the stabilizers on } \mu^{-1}(\xi) \text { are finite } \\
& \Longrightarrow \mu^{-1}(\xi) / G \text { is an orbifold }[91,92]
\end{aligned}
$$

Let $G_{p}$ be the stabilizer of $p$. By the slice theorem (Lecture 23), $\mu^{-1}(\xi) / G$ is modeled by $S / G_{p}$, where $S$ is a $G_{p}$-invariant disk in $\mu^{-1}(\xi)$ through $p$ and transverse to $\mathcal{O}_{p}$. Hence, locally $\mu^{-1}(\xi) / G$ looks indeed like $\mathbb{R}^{n}$ divided by a finite group action.

Example. Consider the $S^{1}$-action on $\mathbb{C}^{2}$ given by $e^{i \theta} \cdot\left(z_{1}, z_{2}\right)=\left(e^{i k \theta} z_{1}, e^{i \theta} z_{2}\right)$ for some fixed integer $k \geq 2$. This is hamiltonian with moment map

$$
\mu: \begin{aligned}
\mathbb{C}^{2} & \longrightarrow \mathbb{R} \\
\left(z_{1}, z_{2}\right) & \longmapsto-\frac{1}{2}\left(k\left|z_{1}\right|^{2}+\left|z_{2}\right|^{2}\right) .
\end{aligned}
$$

Any $\xi<0$ is a regular value and $\mu^{-1}(\xi)$ is a 3 -dimensional ellipsoid. The stabilizer of $\left(z_{1}, z_{2}\right) \in \mu^{-1}(\xi)$ is $\{1\}$ if $z_{2} \neq 0$, and is $\mathbb{Z}_{k}=\left\{e^{i \frac{2 \pi \ell}{k}} \mid \ell=0,1, \ldots, k-1\right\}$ if $z_{2}=0$. The reduced space $\mu^{-1}(\xi) / S^{1}$ is called a teardrop orbifold or conehead; it has one cone (also known as a dunce cap) singularity of type $k$ (with cone angle $\left.\frac{2 \pi}{k}\right)$.

Example. Let $S^{1}$ act on $\mathbb{C}^{2}$ by $e^{i \theta} \cdot\left(z_{1}, z_{2}\right)=\left(e^{i k \theta} z_{1}, e^{i \ell \theta} z_{2}\right)$ for some integers $k, \ell \geq 2$. Suppose that $k$ and $\ell$ are relatively prime. Then

$$
\begin{array}{ccl}
\left(z_{1}, 0\right) & \text { has stabilizer } \mathbb{Z}_{k} & \left(\text { for } z_{1} \neq 0\right) \\
\left(0, z_{2}\right) & \text { has stabilizer } \mathbb{Z}_{\ell} & \left(\text { for } z_{2} \neq 0\right) \\
\left(z_{1}, z_{2}\right) & \text { has stabilizer }\{1\} & \left(\text { for } z_{1}, z_{2} \neq 0\right)
\end{array}
$$


The quotient $\mu^{-1}(\xi) / S^{1}$ is called a football orbifold. It has two cone singularities, one of type $k$ and another of type $\ell$.

Example. More generally, the reduced spaces of $S^{1}$ acting on $\mathbb{C}^{n}$ by

$$
e^{i \theta} \cdot\left(z_{1}, \ldots, z_{n}\right)=\left(e^{i k_{1} \theta} z_{1}, \ldots, e^{i k_{n} \theta} z_{n}\right),
$$

are called weighted (or twisted) projective spaces. 


\section{Homework 18: Spherical Pendulum}

This set of problems is from [53].

The spherical pendulum is a mechanical system consisting of a massless rigid rod of length $l$, fixed at one end, whereas the other end has a plumb bob of mass $m$, which may oscillate freely in all directions. Assume that the force of gravity is constant pointing vertically downwards, and that this is the only external force acting on this system.

Let $\varphi, \theta(0<\varphi<\pi, 0<\theta<2 \pi)$ be spherical coordinates for the bob. For simplicity assume that $m=l=1$.

1. Let $\eta, \xi$ be the coordinates along the fibers of $T^{*} S^{2}$ induced by the spherical coordinates $\varphi, \theta$ on $S^{2}$. Show that the function $H: T^{*} S^{2} \rightarrow \mathbb{R}$ given by

$$
H(\varphi, \theta, \eta, \xi)=\frac{1}{2}\left(\eta^{2}+\frac{\xi^{2}}{(\sin \varphi)^{2}}\right)+\cos \varphi,
$$

is an appropriate hamiltonian function to describe the spherical pendulum.

2. Compute the critical points of the function $H$. Show that, on $S^{2}$, there are exactly two critical points: $s$ (where $H$ has a minimum) and $u$. These points are called the stable and unstable points of $H$, respectively. Justify this terminology, i.e., show that a trajectory whose initial point is close to $s$ stays close to $s$ forever, and show that this is not the case for $u$. What is happening physically?

3. Show that the group of rotations about the vertical axis is a group of symmetries of the spherical pendulum.

Show that, in the coordinates above, the integral of motion associated with these symmetries is the function

$$
J(\varphi, \theta, \eta, \xi)=\xi .
$$

Give a more coordinate-independent description of $J$, one that makes sense also on the cotangent fibers above the North and South poles. 
4. Locate all points $p \in T^{*} S^{2}$ where $d H_{p}$ and $d J_{p}$ are linearly dependent:

(a) Clearly, the two critical points $s$ and $u$ belong to this set. Show that these are the only two points where $d H_{p}=d J_{p}=0$.

(b) Show that, if $x \in S^{2}$ is in the southern hemisphere $\left(x_{3}<0\right)$, then there exist exactly two points, $p_{+}=(x, \eta, \xi)$ and $p_{-}=(x,-\eta,-\xi)$, in the cotangent fiber above $x$ where $d H_{p}$ and $d J_{p}$ are linearly dependent.

(c) Show that $d H_{p}$ and $d J_{p}$ are linearly dependent along the trajectory of the hamiltonian vector field of $H$ through $p_{+}$.

Conclude that this trajectory is also a trajectory of the hamiltonian vector field of $J$, and, hence, that its projection onto $S^{2}$ is a latitudinal circle (of the form $x_{3}=$ constant).

Show that the projection of the trajectory through $p_{-}$is the same latitudinal circle traced in the opposite direction.

5. Show that any nonzero value $j$ is a regular value of $J$, and that $S^{1}$ acts freely on the level set $J=j$. What happens on the cotangent fibers above the North and South poles?

6. For $j \neq 0$ describe the reduced system and sketch the level curves of the reduced hamiltonian.

7. Show that the integral curves of the original system on the level set $J=j$ can be obtained from those of the reduced system by "quadrature", in other words, by a simple integration.

8. Show that the reduced system for $j \neq 0$ has exactly one equilibrium point. Show that the corresponding relative equilibrium for the original system is one of the horizontal curves in exercise 4.

9. The energy-momentum map is the map $(H, J): T^{*} S^{2} \rightarrow \mathbb{R}^{2}$. Show that, if $j \neq 0$, the level set $(H, J)=(h, j)$ of the energy-momentum map is either a circle (in which case it is one of the horizontal curves in exercise 4), or a two-torus. Show that the projection onto the configuration space of the two-torus is an annular region on $S^{2}$. 



\section{Part X}

\section{Moment Maps Revisited}

Moment maps and symplectic reduction have been finding infinite-dimensional incarnations with amazing consequences for differential geometry. Lecture 25 sketches the symplectic approach of Atiyah and Bott to Yang-Mills theory.

Lecture 27 describes the convexity of the image of a torus moment map, one of the most striking geometric characteristics of moment maps.

\section{Moment Map in Gauge Theory}

\subsection{Connections on a Principal Bundle}

Let $G$ be a Lie group and $B$ a manifold.

Definition 25.1 $A$ principal $G$-bundle over $B$ is a manifold $P$ with a smooth map $\pi: P \rightarrow B$ satisfying the following conditions:

(a) $G$ acts freely on $P$ (on the left),

(b) $B$ is the orbit space for this action and $\pi$ is the point-orbit projection, and

(c) there is an open covering of $B$, such that, to each set $\mathcal{U}$ in that covering corresponds a map $\varphi_{\mathcal{U}}: \pi^{-1}(\mathcal{U}) \rightarrow \mathcal{U} \times G$ with

$$
\varphi_{\mathcal{U}}(p)=\left(\pi(p), s_{\mathcal{U}}(p)\right) \quad \text { and } \quad s_{\mathcal{U}}(g \cdot p)=g \cdot s_{\mathcal{U}}(p), \quad \forall p \in \pi^{-1}(\mathcal{U})
$$

The $G$-valued maps $s_{\mathcal{U}}$ are determined by the corresponding $\varphi_{\mathcal{U}}$. Condition (c) is called the property of being locally trivial.

If $P$ with map $\pi: P \rightarrow B$ is a principal $G$-bundle over $B$, then the manifold $B$ is called the base, the manifold $P$ is called the total space, the Lie group $G$ is called the structure group, and the map $\pi$ is called the projection. This principal bundle is also represented by the following diagram:

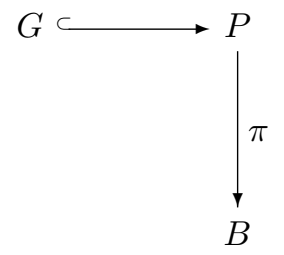

Example. Let $P$ be the 3-sphere regarded as unit vectors in $\mathbb{C}^{2}$ :

$$
P=S^{3}=\left\{\left(z_{1}, z_{2}\right) \in \mathbb{C}^{2}:\left|z_{1}\right|^{2}+\left|z_{2}\right|^{2}=1\right\} .
$$


Let $G$ be the circle group, where $e^{i \theta} \in S^{1}$ acts on $S^{3}$ by complex multiplication,

$$
\left(z_{1}, z_{2}\right) \longmapsto\left(e^{i \theta} z_{1}, e^{i \theta} z_{2}\right) .
$$

Then the quotient space $B$ is the first complex projective space, that is, the twosphere. This data forms a principal $S^{1}$-bundle, known as the Hopf fibration:

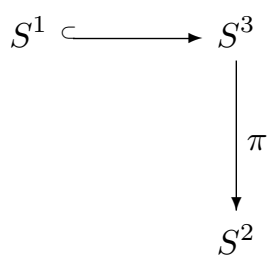

An action $\psi: G \rightarrow \operatorname{Diff}(P)$ induces an infinitesimal action

$$
\begin{aligned}
& d \psi: \quad \mathfrak{g} \longrightarrow \chi(P) \\
& X \longmapsto X^{\#}=\text { vector field generated by the } \\
& \text { one-parameter group }\{\exp t X(e) \mid t \in \mathbb{R}\} \text {. }
\end{aligned}
$$

From now on, fix a basis $X_{1}, \ldots, X_{k}$ of $\mathfrak{g}$.

Let $P$ be a principal $G$-bundle over $B$. Since the $G$-action is free, the vector fields $X_{1}^{\#}, \ldots, X_{k}^{\#}$ are linearly independent at each $p \in P$. The vertical bundle $V$ is the rank $k$ subbundle of $T P$ generated by $X_{1}^{\#}, \ldots, X_{k}^{\#}$.

Exercise. Check that the vertical bundle $V$ is the set of vectors tangent to $P$ which lie in the kernel of the derivative of the bundle projection $\pi$. (This shows that $V$ is independent of the choice of basis for $\mathfrak{g}$.)

Definition 25.2 A (Ehresmann) connection on a principal bundle $P$ is a choice of a splitting

$$
T P=V \oplus H,
$$

where $H$ is a $G$-invariant subbundle of $T P$ complementary to the vertical bundle $V$. The bundle $H$ is called the horizontal bundle.

\subsection{Connection and Curvature Forms}

A connection on a principal bundle $P$ may be equivalently described in terms of 1 -forms.

Definition 25.3 A connection form on a principal bundle $P$ is a Lie-algebra-valued 1-form

$$
A=\sum_{i=1}^{k} A_{i} \otimes X_{i} \quad \in \Omega^{1}(P) \otimes \mathfrak{g}
$$

such that: 
(a) $A$ is $G$-invariant, with respect to the product action of $G$ on $\Omega^{1}(P)$ (induced by the action on $P$ ) and on $\mathfrak{g}$ (the adjoint representation), and

(b) $A$ is vertical, in the sense that $\imath_{X \#} A=X$ for any $X \in \mathfrak{g}$.

Exercise. Show that a connection $T P=V \oplus H$ determines a connection form $A$ and vice-versa by the formula

$$
H=\operatorname{ker} A=\left\{v \in T P \mid \iota_{v} A=0\right\} .
$$

Given a connection on $P$, the splitting $T P=V \oplus H$ induces the following splittings for bundles:

$$
\begin{aligned}
T^{*} P & =V^{*} \oplus H^{*} \\
\wedge^{2} T^{*} P & =\left(\wedge^{2} V^{*}\right) \oplus\left(V^{*} \wedge H^{*}\right) \oplus\left(\wedge^{2} H^{*}\right)
\end{aligned}
$$

and for their sections:

$$
\begin{aligned}
& \Omega^{1}(P)=\Omega_{\text {vert }}^{1}(P) \oplus \Omega_{\text {horiz }}^{1}(P) \\
& \Omega^{2}(P)=\Omega_{\text {vert }}^{2}(P) \oplus \Omega_{\text {mix }}^{2}(P) \oplus \Omega_{\text {horiz }}^{2}(P)
\end{aligned}
$$

The corresponding connection form $A$ is in $\Omega_{\text {vert }}^{1} \otimes \mathfrak{g}$. Its exterior derivative $d A$ is in

$$
\Omega^{2}(P) \otimes \mathfrak{g}=\left(\Omega_{\text {vert }}^{2} \oplus \Omega_{\text {mix }}^{2} \oplus \Omega_{\text {horiz }}^{2}\right) \otimes \mathfrak{g},
$$

and thus decomposes into three components,

$$
d A=(d A)_{\text {vert }}+(d A)_{\text {mix }}+(d A)_{\text {horiz }} .
$$

Exercise. Check that:

(a) $(d A)_{\text {vert }}(X, Y)=[X, Y]$, i.e., $(d A)_{\text {vert }}=\frac{1}{2} \sum_{i, \ell, m} c_{\ell m}^{i} A_{\ell} \wedge A_{m} \otimes X_{i}$, where the $c_{\ell m}^{i}$ 's are the structure constants of the Lie algebra with respect to the chosen basis, and defined by $\left[X_{\ell}, X_{m}\right]=\sum_{i, \ell, m} c_{\ell m}^{i} X_{i}$;

(b) $(d A)_{\text {mix }}=0$.

According to the previous exercise, the relevance of $d A$ may come only from its horizontal component.

Definition 25.4 The curvature form of a connection is the horizontal component of its connection form. I.e., if $A$ is the connection form, then

$$
\text { curv } A=(d A)_{\text {horiz }} \quad \in \Omega_{\text {horiz }}^{2} \otimes \mathfrak{g} \text {. }
$$

Definition 25.5 A connection is called flat if its curvature is zero. 


\subsection{Symplectic Structure on the Space of Connections}

Let $P$ be a principal $G$-bundle over $B$. If $A$ is a connection form on $P$, and if $a \in \Omega_{\text {horiz }}^{1} \otimes \mathfrak{g}$ is $G$-invariant for the product action, then it is easy to check that $A+a$ is also a connection form on $P$. Reciprocally, any two connection forms on $P$ differ by an $a \in\left(\Omega_{\text {horiz }}^{1} \otimes \mathfrak{g}\right)^{G}$. We conclude that the set $\mathcal{A}$ of all connections on the principal $G$-bundle $P$ is an affine space modeled on the linear space

$$
\mathfrak{a}=\left(\Omega_{\text {horiz }}^{1} \otimes \mathfrak{g}\right)^{G} .
$$

Now let $P$ be a principal $G$-bundle over a compact oriented 2-dimensional riemannian manifold $B$ (for instance, $B$ is a Riemann surface). Suppose that the group $G$ is compact or semisimple. Atiyah and Bott [7] noticed that the corresponding space $\mathcal{A}$ of all connections may be treated as an infinite-dimensional symplectic manifold. This will require choosing a $G$-invariant inner product $\langle\cdot, \cdot\rangle$ on $\mathfrak{g}$, which always exists, either by averaging any inner product when $G$ is compact, or by using the Killing form on semisimple groups.

Since $\mathcal{A}$ is an affine space, its tangent space at any point $A$ is identified with the model linear space $\mathfrak{a}$. With respect to a basis $X_{1}, \ldots, X_{k}$ for the Lie algebra $\mathfrak{g}$, elements $a, b \in \mathfrak{a}^{14}$ are written

$$
a=\sum a_{i} \otimes X_{i} \quad \text { and } \quad b=\sum b_{i} \otimes X_{i} .
$$

If we wedge $a$ and $b$, and then integrate over $B$ using the riemannian volume, we obtain a real number:

$$
\begin{aligned}
\omega: \mathfrak{a} \times \mathfrak{a} & \longrightarrow\left(\Omega_{\text {horiz }}^{2}(P)\right)^{G} \simeq \Omega^{2}(B) \\
(a, b) & \longmapsto \mathbb{R} \\
\sum_{i, j} a_{i} \wedge b_{j}\left\langle X_{i}, X_{j}\right\rangle & \longmapsto \int_{B} \sum_{i, j} a_{i} \wedge b_{j}\left\langle X_{i}, X_{j}\right\rangle .
\end{aligned}
$$

We have used that the pullback $\pi^{*}: \Omega^{2}(B) \rightarrow \Omega^{2}(P)$ is an isomorphism onto its image $\left(\Omega_{\text {horiz }}^{2}(P)\right)^{G}$.

Exercise. Show that if $\omega(a, b)=0$ for all $b \in \mathfrak{a}$, then $a$ must be zero.

The map $\omega$ is nondegenerate, skew-symmetric, bilinear and constant in the sense that it does not depend on the base point $A$. Therefore, it has the right to be called a symplectic form on $\mathcal{A}$, so the pair $(\mathcal{A}, \omega)$ is an infinite-dimensional symplectic manifold.

\subsection{Action of the Gauge Group}

Let $P$ be a principal $G$-bundle over $B$. A diffeomorphism $f: P \rightarrow P$ commuting with the $G$-action determines a diffeomorphism $f_{\text {basic }}: B \rightarrow B$ by projection.

\footnotetext{
${ }^{14}$ The choice of symbols is in honor of Atiyah and Bott!
} 
Definition 25.6 A diffeomorphism $f: P \rightarrow P$ commuting with the $G$-action is a gauge transformation if the induced $f_{\text {basic }}$ is the identity. The gauge group of $P$ is the group $\mathcal{G}$ of all gauge transformations of $P$.

The derivative of an $f \in \mathcal{G}$ takes a connection $T P=V \oplus H$ to another connection $T P=V \oplus H_{f}$, and thus induces an action of $\mathcal{G}$ in the space $\mathcal{A}$ of all connections. Recall that $\mathcal{A}$ has a symplectic form $\omega$. Atiyah and Bott [7] noticed that the action of $\mathcal{G}$ on $(\mathcal{A}, \omega)$ is hamiltonian, where the moment map (appropriately interpreted) is the map

$$
\begin{aligned}
\mu: \mathcal{A} & \longrightarrow\left(\Omega^{2}(P) \otimes \mathfrak{g}\right)^{G} \\
A & \longmapsto \operatorname{curv} A,
\end{aligned}
$$

i.e., the moment map "is" the curvature! We will describe this construction in detail for the case of circle bundles in the next section.

Remark. The reduced space at level zero

$$
\mathcal{M}=\mu^{-1}(0) / \mathcal{G}
$$

is the space of flat connections modulo gauge equivalence, known as the moduli space of flat connections. It turns out that $\mathcal{M}$ is a finite-dimensional symplectic orbifold.

\subsection{Case of Circle Bundles}

What does the Atiyah-Bott construction of the previous section look like for the case when $G=S^{1}$ ?

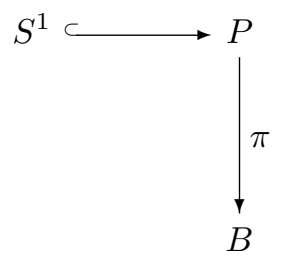

Let $v$ be the generator of the $S^{1}$-action on $P$, corresponding to the basis 1 of $\mathfrak{g} \simeq \mathbb{R}$. A connection form on $P$ is a usual 1-form $A \in \Omega^{1}(P)$ such that

$$
\mathcal{L}_{v} A=0 \quad \text { and } \quad \imath_{v} A=1 .
$$

If we fix one particular connection $A_{0}$, then any other connection is of the form $A=A_{0}+a$ for some $a \in \mathfrak{a}=\left(\Omega_{\text {horiz }}^{1}(P)\right)^{G}=\Omega^{1}(B)$. The symplectic form on $\mathfrak{a}=\Omega^{1}(B)$ is simply

$$
\begin{aligned}
\omega: \mathfrak{a} \times \mathfrak{a} & \longrightarrow \mathbb{R} \\
(a, b) & \longmapsto \int_{B} \underbrace{a \wedge b}_{\in \Omega^{2}(B)} .
\end{aligned}
$$


The gauge group is $\mathcal{G}=\operatorname{Maps}\left(B, S^{1}\right)$, because a gauge transformation is multiplication by some element of $S^{1}$ over each point in $B$ :

$$
\begin{aligned}
& \phi: \quad \mathcal{G} \longrightarrow \operatorname{Diff}(P) \\
& h: B \rightarrow S^{1} \longmapsto \quad \phi_{h}: \quad P \rightarrow P \\
& p \rightarrow h(\pi(p)) \cdot p
\end{aligned}
$$

The Lie algebra of $\mathcal{G}$ is

$$
\text { Lie } \mathcal{G}=\operatorname{Maps}(B, \mathbb{R})=C^{\infty}(B) .
$$

Its dual space is

$$
(\text { Lie } \mathcal{G})^{*}=\Omega^{2}(B)
$$

where the duality is provided by integration over $B$

$$
\begin{aligned}
C^{\infty}(B) \times \Omega^{2}(B) & \longrightarrow \mathbb{R} \\
(h, \beta) & \longmapsto \int_{B} h \beta .
\end{aligned}
$$

(it is topological or smooth duality, as opposed to algebraic duality).

The gauge group acts on the space of all connections by

$$
\begin{aligned}
\mathcal{G} & \longrightarrow \operatorname{Diff}(\mathcal{A}) \\
h(x)=e^{i \theta(x)} & \longmapsto(A \mapsto A \underbrace{-\pi^{*} d \theta}_{\in \mathfrak{a}})
\end{aligned}
$$

Exercise. Check the previous assertion about the action on connections.

Hint: First deal with the case where $P=S^{1} \times B$ is a trivial bundle, in which case $h \in \mathcal{G}$ acts on $P$ by

$$
\phi_{h}:(t, x) \longmapsto(t+\theta(x), x),
$$

and where every connection can be written $A=d t+\beta$, with $\beta \in \Omega^{1}(B)$. A gauge transformation $h \in \mathcal{G}$ acts on $\mathcal{A}$ by

$$
A \longmapsto \phi_{h^{-1}}^{*}(A)
$$

The infinitesimal action of $\mathcal{G}$ on $\mathcal{A}$ is

$$
\begin{aligned}
& d \phi: \quad \text { Lie } \mathcal{G} \longrightarrow \chi(\mathcal{A}) \\
& X \longmapsto X^{\#}=\text { vector field described by the transformation } \\
& (A \mapsto A \underbrace{-d X}_{\in \Omega^{1}(B)=\mathfrak{a}})
\end{aligned}
$$

so that $X^{\#}=-d X$. 
Finally, we will check that

$$
\begin{aligned}
\mu: \mathcal{A} & \longrightarrow(\operatorname{Lie} \mathcal{G})^{*}=\Omega^{2}(B) \\
A & \longmapsto \operatorname{curv} A
\end{aligned}
$$

is indeed a moment map for the action of the gauge group on $\mathcal{A}$.

Exercise. Check that in this case:

(a) $\operatorname{curv} A=d A \in\left(\Omega_{\text {horiz }}^{2}(P)\right)^{G}=\Omega^{2}(B)$,

(b) $\mu$ is $\mathcal{G}$-invariant.

The previous exercise takes care of the equivariance condition, since the action of $\mathcal{G}$ on $\Omega^{2}(B)$ is trivial.

Take any $X \in$ Lie $\mathcal{G}=C^{\infty}(B)$. We need to check that

$$
d \mu^{X}(a)=\omega\left(X^{\#}, a\right), \quad \forall a \in \Omega^{1}(B) .
$$

As for the left-hand side of $(\star)$, the map $\mu^{X}$,

$$
\begin{aligned}
\mu^{X}: \mathcal{A} & \longrightarrow \mathbb{R} \\
A & \longmapsto\langle\underbrace{X}_{\in C^{\infty}(B)}, \underbrace{d A}_{\in \Omega^{2}(B)}\rangle=\int_{B} X \cdot d A,
\end{aligned}
$$

is linear in $A$. Consequently,

$$
\begin{aligned}
d \mu^{X}: \mathfrak{a} & \longrightarrow \mathbb{R} \\
a & \longmapsto \int_{B} X \cdot d a .
\end{aligned}
$$

As for the right-hand side of $(\star)$, by definition of $\omega$, we have

$$
\omega\left(X^{\#}, a\right)=\int_{B} X^{\#} \cdot a=-\int_{B} d X \cdot a .
$$

But, by Stokes theorem, the last integral is

$$
-\int_{B} d X \cdot a=\int_{B} X \cdot d a
$$

so we are done in proving that $\mu$ is the moment map. 


\section{Homework 19: Examples of Moment Maps}

1. Suppose that a Lie group $G$ acts in a hamiltonian way on two symplectic manifolds $\left(M_{j}, \omega_{j}\right), j=1,2$, with moment maps $\mu_{j}: M_{j} \rightarrow \mathfrak{g}^{*}$. Prove that the diagonal action of $G$ on $M_{1} \times M_{2}$ is hamiltonian with moment map $\mu: M_{1} \times M_{2} \rightarrow \mathfrak{g}^{*}$ given by

$$
\mu\left(p_{1}, p_{2}\right)=\mu_{1}\left(p_{1}\right)+\mu_{2}\left(p_{2}\right), \text { for } p_{j} \in M_{j} .
$$

2. Let $\mathbb{T}^{n}=\left\{\left(t_{1}, \ldots, t_{n}\right) \in \mathbb{C}^{n}:\left|t_{j}\right|=1\right.$, for all $\left.j\right\}$ be a torus acting on $\mathbb{C}^{n}$ by

$$
\left(t_{1}, \ldots, t_{n}\right) \cdot\left(z_{1}, \ldots, z_{n}\right)=\left(t_{1}^{k_{1}} z_{1}, \ldots, t_{n}^{k_{n}} z_{n}\right),
$$

where $k_{1}, \ldots, k_{n} \in \mathbb{Z}$ are fixed. Check that this action is hamiltonian with moment map $\mu: \mathbb{C}^{n} \rightarrow\left(\mathfrak{t}^{n}\right)^{*} \simeq \mathbb{R}^{n}$ given by

$$
\mu\left(z_{1}, \ldots, z_{n}\right)=-\frac{1}{2}\left(k_{1}\left|z_{1}\right|^{2}, \ldots, k_{n}\left|z_{n}\right|^{2}\right)(+ \text { constant }) .
$$

3. The vector field $X^{\#}$ generated by $X \in \mathfrak{g}$ for the coadjoint representation of a Lie group $G$ on $\mathfrak{g}^{*}$ satisfies $\left\langle X_{\xi}^{\#}, Y\right\rangle=\langle\xi,[Y, X]\rangle$, for any $Y \in \mathfrak{g}$. Equip the coadjoint orbits with the canonical symplectic forms. Show that, for each $\xi \in \mathfrak{g}^{*}$, the coadjoint action on the orbit $G \cdot \xi$ is hamiltonian with moment map the inclusion map:

$$
\mu: G \cdot \xi \hookrightarrow \mathfrak{g}^{*}
$$

4. Consider the natural action of $\mathrm{U}(n)$ on $\left(\mathbb{C}^{n}, \omega_{0}\right)$. Show that this action is hamiltonian with moment map $\mu: \mathbb{C}^{n} \rightarrow \mathfrak{u}(n)$ given by

$$
\mu(z)=\frac{i}{2} z z^{*},
$$

where we identify the Lie algebra $\mathfrak{u}(n)$ with its dual via the inner product $(A, B)=\operatorname{trace}\left(A^{*} B\right)$.

Hint: Denote the elements of $\mathrm{U}(n)$ in terms of real and imaginary parts $g=$ $h+i k$. Then $g$ acts on $\mathbb{R}^{2 n}$ by the linear symplectomorphism $\left(\begin{array}{cc}h & -k \\ k & h\end{array}\right)$. The Lie algebra $\mathfrak{u}(n)$ is the set of skew-hermitian matrices $X=V+i W$ where $V=-V^{t} \in \mathbb{R}^{n \times n}$ and $W=W^{t} \in \mathbb{R}^{n \times n}$. Show that the infinitesimal action is generated by the hamiltonian functions

$$
\mu^{X}(z)=-\frac{1}{2}(x, W x)+(y, V x)-\frac{1}{2}(y, W y)
$$

where $z=x+i y, x, y \in \mathbb{R}^{n}$ and $(\cdot, \cdot)$ is the standard inner product. Show that

$$
\mu^{X}(z)=\frac{1}{2} i z^{*} X z=\frac{1}{2} i \operatorname{trace}\left(z z^{*} X\right) .
$$

Check that $\mu$ is equivariant. 
5. Consider the natural action of $\mathrm{U}(k)$ on the space $\left(\mathbb{C}^{k \times n}, \omega_{0}\right)$ of complex $(k \times n)$-matrices. Identify the Lie algebra $\mathfrak{u}(k)$ with its dual via the inner product $(A, B)=\operatorname{trace}\left(A^{*} B\right)$. Prove that a moment map for this action is given by

$$
\mu(A)=\frac{i}{2} A A^{*}+\frac{\mathrm{Id}}{2 i}, \text { for } A \in \mathbb{C}^{k \times n} .
$$

(The choice of the constant $\frac{\mathrm{Id}}{2 i}$ is for convenience in Homework 20.)

Hint: Exercises 1 and 4.

6. Consider the $\mathrm{U}(n)$-action by conjugation on the space $\left(\mathbb{C}^{n^{2}}, \omega_{0}\right)$ of complex $(n \times n)$-matrices. Show that a moment map for this action is given by

$$
\mu(A)=\frac{i}{2}\left[A, A^{*}\right] .
$$

Hint: Previous exercise and its "transpose" version. 


\section{Existence and Uniqueness of Moment Maps}

\subsection{Lie Algebras of Vector Fields}

Let $(M, \omega)$ be a symplectic manifold and $v \in \chi(M)$ a vector field on $M$.

$$
\begin{aligned}
v \text { is symplectic } & \Longleftrightarrow v_{v} \omega \text { is closed }, \\
v \text { is hamiltonian } & \Longleftrightarrow v_{v} \omega \text { is exact . }
\end{aligned}
$$

The spaces

$$
\begin{aligned}
\chi^{\text {sympl }}(M) & =\text { symplectic vector fields on } M, \\
\chi^{\text {ham }}(M) & =\text { hamiltonian vector fields on } M .
\end{aligned}
$$

are Lie algebras for the Lie bracket of vector fields. $C^{\infty}(M)$ is a Lie algebra for the Poisson bracket, $\{f, g\}=\omega\left(v_{f}, v_{g}\right) . H^{1}(M ; \mathbb{R})$ and $\mathbb{R}$ are regarded as Lie algebras for the trivial bracket. We have two exact sequences of Lie algebras:

$$
\begin{aligned}
& 0 \longrightarrow \chi^{\operatorname{ham}}(M) \hookrightarrow \chi^{\mathrm{sympl}}(M) \longrightarrow H^{1}(M ; \mathbb{R}) \longrightarrow 0 \\
& v \longmapsto\left[\imath_{v} \omega\right] \\
& 0 \longrightarrow \mathbb{R} \quad \hookrightarrow \quad C^{\infty}(M) \longrightarrow \chi^{\mathrm{ham}}(M) \longrightarrow 0 \\
& f \longmapsto v_{f} \text {. }
\end{aligned}
$$

In particular, if $H^{1}(M ; \mathbb{R})=0$, then $\chi^{\text {ham }}(M)=\chi^{\text {sympl }}(M)$.

Let $G$ be a connected Lie group. A symplectic action $\psi: G \rightarrow \operatorname{Sympl}(M, \omega)$ induces an infinitesimal action

$$
\begin{aligned}
& d \psi: \mathfrak{g} \longrightarrow \chi^{\text {sympl }}(M) \\
& X \longmapsto X^{\#}=\text { vector field generated by the } \\
& \text { one-parameter group }\{\exp t X(e) \mid t \in \mathbb{R}\} \text {. }
\end{aligned}
$$

Exercise. Check that the map $d \psi$ is a Lie algebra anti-homomorphism.

The action $\psi$ is hamiltonian if and only if there is a Lie algebra homomorphism $\mu^{*}: \mathfrak{g} \rightarrow C^{\infty}(M)$ lifting $d \psi$, i.e., making the following diagram commute.

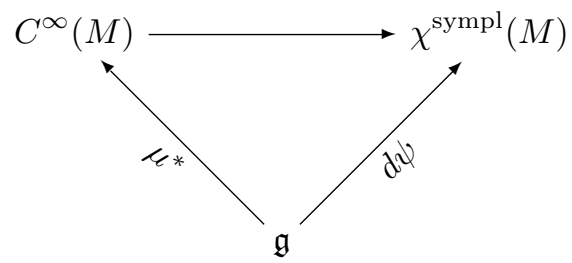

The map $\mu^{*}$ is then called a comoment map (defined in Lecture 22).

$$
\begin{aligned}
& \begin{array}{l}
\text { Existence of } \mu^{*} \\
\text { comoment map }
\end{array} \Longleftrightarrow \begin{array}{l}
\text { Existence of } \mu \\
\text { moment map }
\end{array}
\end{aligned}
$$

Lie algebra homomorphism $\longleftrightarrow$ equivariance 


\subsection{Lie Algebra Cohomology}

Let $\mathfrak{g}$ be a Lie algebra, and

$$
\begin{aligned}
C^{k} & :=\Lambda^{k} \mathfrak{g}^{*}=k \text {-cochains on } \mathfrak{g} \\
& =\text { alternating } k \text {-linear maps } \underbrace{\mathfrak{g} \times \ldots \times \mathfrak{g}}_{k} \longrightarrow \mathbb{R} .
\end{aligned}
$$

Define a linear operator $\delta: C^{k} \rightarrow C^{k+1}$ by

$$
\delta c\left(X_{0}, \ldots, X_{k}\right)=\sum_{i<j}(-1)^{i+j} c\left(\left[X_{i}, X_{j}\right], X_{0}, \ldots, \widehat{X}_{i}, \ldots, \widehat{X}_{j}, \ldots, X_{k}\right)
$$

Exercise. Check that $\delta^{2}=0$.

The Lie algebra cohomology groups (or Chevalley cohomology groups) of $\mathfrak{g}$ are the cohomology groups of the complex $0 \stackrel{\delta}{\rightarrow} C^{0} \stackrel{\delta}{\rightarrow} C^{1} \stackrel{\delta}{\rightarrow} \ldots$ :

$$
H^{k}(\mathfrak{g} ; \mathbb{R}):=\frac{\operatorname{ker} \delta: C^{k} \longrightarrow C^{k+1}}{\operatorname{im} \delta: C^{k-1} \longrightarrow C^{k}}
$$

Theorem 26.1 If $\mathfrak{g}$ is the Lie algebra of a compact connected Lie group $G$, then

$$
H^{k}(\mathfrak{g} ; \mathbb{R})=H_{\mathrm{deRham}}^{k}(G)
$$

Proof. Exercise. Hint: by averaging show that the de Rham cohomology can be computed from the subcomplex of $G$-invariant forms.

Meaning of $H^{1}(\mathfrak{g} ; \mathbb{R})$ and $H^{2}(\mathfrak{g} ; \mathbb{R})$ :

- An element of $C^{1}=\mathfrak{g}^{*}$ is a linear functional on $\mathfrak{g}$. If $c \in \mathfrak{g}^{*}$, then $\delta c\left(X_{0}, X_{1}\right)=$ $-c\left(\left[X_{0}, X_{1}\right]\right)$. The commutator ideal of $\mathfrak{g}$ is

$$
[\mathfrak{g}, \mathfrak{g}]:=\{\text { linear combinations of }[X, Y] \text { for any } X, Y \in \mathfrak{g}\} .
$$

Since $\delta c=0$ if and only if $c$ vanishes on $[\mathfrak{g}, \mathfrak{g}]$, we conclude that

$$
H^{1}(\mathfrak{g} ; \mathbb{R})=[\mathfrak{g}, \mathfrak{g}]^{0}
$$

where $[\mathfrak{g}, \mathfrak{g}]^{0} \subseteq \mathfrak{g}^{*}$ is the annihilator of $[\mathfrak{g}, \mathfrak{g}]$.

- An element of $C^{2}$ is an alternating bilinear map $c: \mathfrak{g} \times \mathfrak{g} \rightarrow \mathbb{R}$.

$$
\delta c\left(X_{0}, X_{1}, X_{2}\right)=-c\left(\left[X_{0}, X_{1}\right], X_{2}\right)+c\left(\left[X_{0}, X_{2}\right], X_{1}\right)-c\left(\left[X_{1}, X_{2}\right], X_{0}\right) .
$$

If $c=\delta b$ for some $b \in C^{1}$, then

$$
c\left(X_{0}, X_{1}\right)=(\delta b)\left(X_{0}, X_{1}\right)=-b\left(\left[X_{0}, X_{1}\right]\right) .
$$




\subsection{Existence of Moment Maps}

Theorem 26.2 If $H^{1}(\mathfrak{g} ; \mathbb{R})=H^{2}(\mathfrak{g}, \mathbb{R})=0$, then any symplectic $G$-action is hamiltonian.

Proof. Let $\psi: G \rightarrow \operatorname{Sympl}(M, \omega)$ be a symplectic action of $G$ on a symplectic manifold $(M, \omega)$. Since

$$
H^{1}(\mathfrak{g} ; \mathbb{R})=0 \Longleftrightarrow[\mathfrak{g}, \mathfrak{g}]=\mathfrak{g}
$$

and since commutators of symplectic vector fields are hamiltonian, we have

$$
d \psi: \mathfrak{g}=[\mathfrak{g}, \mathfrak{g}] \longrightarrow \chi^{\operatorname{ham}}(M) .
$$

The action $\psi$ is hamiltonian if and only if there is a Lie algebra homomorphism $\mu^{*}: \mathfrak{g} \rightarrow C^{\infty}(M)$ such that the following diagram commutes.

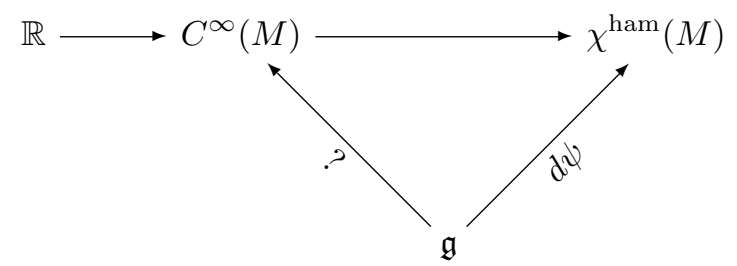

We first take an arbitrary vector space lift $\tau: \mathfrak{g} \rightarrow C^{\infty}(M)$ making the diagram commute, i.e., for each basis vector $X \in \mathfrak{g}$, we choose

$$
\tau(X)=\tau^{X} \in C^{\infty}(M) \quad \text { such that } \quad v_{\left(\tau^{X}\right)}=d \psi(X) .
$$

The map $X \mapsto \tau^{X}$ may not be a Lie algebra homomorphism. By construction, $\tau^{[X, Y]}$ is a hamiltonian function for $[X, Y]^{\#}$, and (as computed in Lecture 16) $\left\{\tau^{X}, \tau^{Y}\right\}$ is a hamiltonian function for $-\left[X^{\#}, Y^{\#}\right]$. Since $[X, Y]^{\#}=-\left[X^{\#}, Y^{\#}\right]$, the corresponding hamiltonian functions must differ by a constant:

$$
\tau^{[X, Y]}-\left\{\tau^{X}, \tau^{Y}\right\}=c(X, Y) \in \mathbb{R} .
$$

By the Jacobi identity, $\delta c=0$. Since $H^{2}(\mathfrak{g} ; \mathbb{R})=0$, there is $b \in \mathfrak{g}^{*}$ satisfying $c=\delta b, c(X, Y)=-b([X, Y])$. We define

$$
\begin{aligned}
\mu^{*}: \mathfrak{g} & \longrightarrow C^{\infty}(M) \\
X & \longmapsto \mu^{*}(X)=\tau^{X}+b(X)=\mu^{X} .
\end{aligned}
$$

Now $\mu^{*}$ is a Lie algebra homomorphism:

$$
\mu^{*}([X, Y])=\tau^{[X, Y]}+b([X, Y])=\left\{\tau^{X}, \tau^{Y}\right\}=\left\{\mu^{X}, \mu^{Y}\right\} .
$$

So when is $H^{1}(\mathfrak{g} ; \mathbb{R})=H^{2}(\mathfrak{g} ; \mathbb{R})=0$ ? 
A compact Lie group $G$ is semisimple if $\mathfrak{g}=[\mathfrak{g}, \mathfrak{g}]$.

Examples. The unitary group $\mathrm{U}(n)$ is not semisimple because the multiples of the identity, $S^{1} \cdot \mathrm{Id}$, form a nontrivial center; at the level of the Lie algebra, this corresponds to the 1 -dimensional subspace $\mathbb{R} \cdot \operatorname{Id}$ of scalar matrices which are not commutators since they are not traceless.

Any direct product of the other compact classical groups $\mathrm{SU}(n), \mathrm{SO}(n)$ and $\operatorname{Sp}(n)$ is semisimple $(n>1)$. Any commutative Lie group is not semisimple.

Theorem 26.3 (Whitehead Lemmas) Let $G$ be a compact Lie group.

$$
G \text { is semisimple } \quad \Longleftrightarrow \quad H^{1}(\mathfrak{g} ; \mathbb{R})=H^{2}(\mathfrak{g} ; \mathbb{R})=0 .
$$

A proof can be found in [67, pages 93-95].

Corollary 26.4 If $G$ is semisimple, then any symplectic $G$-action is hamiltonian.

\subsection{Uniqueness of Moment Maps}

Let $G$ be a compact connected Lie group.

Theorem 26.5 If $H^{1}(\mathfrak{g} ; \mathbb{R})=0$, then moment maps for hamiltonian $G$-actions are unique.

Proof. Suppose that $\mu_{1}^{*}$ and $\mu_{2}^{*}$ are two comoment maps for an action $\psi$ :

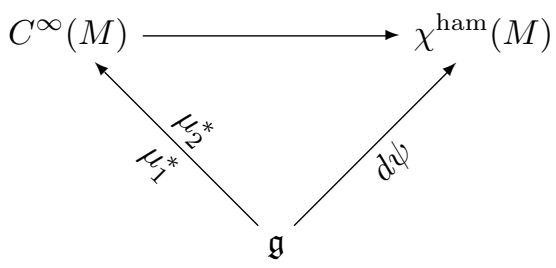

For each $X \in \mathfrak{g}, \mu_{1}^{X}$ and $\mu_{2}^{X}$ are both hamiltonian functions for $X^{\#}$, thus $\mu_{1}^{X}-$ $\mu_{2}^{X}=c(X)$ is locally constant. This defines $c \in \mathfrak{g}^{*}, X \mapsto c(X)$.

Since $\mu_{1}^{*}, \mu_{2}^{*}$ are Lie algebra homomorphisms, we have $c([X, Y])=0, \forall X, Y \in \mathfrak{g}$, i.e., $c \in[\mathfrak{g}, \mathfrak{g}]^{0}=\{0\}$. Hence, $\mu_{1}^{*}=\mu_{2}^{*}$.

Corollary of this proof. In general, if $\mu: M \rightarrow \mathfrak{g}^{*}$ is a moment map, then given any $c \in[\mathfrak{g}, \mathfrak{g}]^{0}, \mu_{1}=\mu+c$ is another moment map.

In other words, moment maps are unique up to elements of the dual of the Lie algebra which annihilate the commutator ideal.

The two extreme cases are:

$G$ semisimple: any symplectic action is hamiltonian , moment maps are unique.

$G$ commutative: symplectic actions may not be hamiltonian , moment maps are unique up to any constant $c \in \mathfrak{g}^{*}$.

Example. The circle action on $\left(\mathbb{T}^{2}, \omega=d \theta_{1} \wedge d \theta_{2}\right)$ by rotations in the $\theta_{1}$ direction has vector field $X^{\#}=\frac{\partial}{\partial \theta_{1}}$; this is a symplectic action but is not hamiltonian. 


\section{Homework 20: Examples of Reduction}

1. For the natural action of $\mathrm{U}(k)$ on $\mathbb{C}^{k \times n}$ with moment map computed in exercise 5 of Homework 19 , we have $\mu^{-1}(0)=\left\{A \in \mathbb{C}^{k \times n} \mid A A^{*}=\mathrm{Id}\right\}$. Show that the quotient

$$
\mu^{-1}(0) / \mathrm{U}(k)=\mathbb{G}(k, n)
$$

is the grassmannian of $k$-planes in $\mathbb{C}^{n}$.

2. Consider the $S^{1}$-action on $\left(\mathbb{R}^{2 n+2}, \omega_{0}\right)$ which, under the usual identification of $\mathbb{R}^{2 n+2}$ with $\mathbb{C}^{n+1}$, corresponds to multiplication by $e^{i t}$. This action is hamiltonian with a moment map $\mu: \mathbb{C}^{n+1} \rightarrow \mathbb{R}$ given by

$$
\mu(z)=-\frac{1}{2}|z|^{2}+\frac{1}{2} .
$$

Prove that the reduction $\mu^{-1}(0) / S^{1}$ is $\mathbb{C} \mathbb{P}^{n}$ with the Fubini-Study symplectic form $\omega_{\text {red }}=\omega_{\mathrm{FS}}$.

Hint: Let pr: $\mathbb{C}^{n+1} \backslash\{0\} \rightarrow \mathbb{C P}^{n}$ denote the standard projection. Check that

$$
\operatorname{pr}^{*} \omega_{\mathrm{FS}}=\frac{i}{2} \partial \bar{\partial} \log \left(|z|^{2}\right) \text {. }
$$

Prove that this form has the same restriction to $S^{2 n+1}$ as $\omega_{0}$.

3. Show that the natural actions of $\mathbb{T}^{n+1}$ and $\mathrm{U}(n+1)$ on $\left(\mathbb{C P}^{n}, \omega_{\mathrm{FS}}\right)$ are hamiltonian, and find formulas for their moment maps.

Hint: Previous exercise and exercises 2 and 4 of Homework 19. 


\section{Convexity}

\subsection{Convexity Theorem}

From now on, we will concentrate on actions of a torus $G=\mathbb{T}^{m}=\mathbb{R}^{m} / \mathbb{Z}^{m}$.

\section{Theorem 27.1 (Atiyah [6], Guillemin-Sternberg [57])}

Let $(M, \omega)$ be a compact connected symplectic manifold, and let $\mathbb{T}^{m}$ be an $m$ torus. Suppose that $\psi: \mathbb{T}^{m} \rightarrow \operatorname{Sympl}(M, \omega)$ is a hamiltonian action with moment map $\mu: M \rightarrow \mathbb{R}^{m}$. Then:

1. the levels of $\mu$ are connected;

2. the image of $\mu$ is convex;

3. the image of $\mu$ is the convex hull of the images of the fixed points of the action.

The image $\mu(M)$ of the moment map is hence called the moment polytope.

Proof. This proof (due to Atiyah) involves induction over $m=\operatorname{dim} \mathbb{T}^{m}$. Consider the statements:

$A_{m}$ : "the levels of $\mu$ are connected, for any $\mathbb{T}^{m}$-action;"

$B_{m}$ : "the image of $\mu$ is convex, for any $\mathbb{T}^{m}$-action."

Then

$$
\begin{aligned}
& (1) \Longleftrightarrow A_{m} \text { holds for all } m, \\
& (2) \Longleftrightarrow B_{m} \text { holds for all } m .
\end{aligned}
$$

- $A_{1}$ is a non-trivial result in Morse theory.

- $A_{m-1} \Longrightarrow A_{m}$ (induction step) is in Homework 21 .

- $B_{1}$ is trivial because in $\mathbb{R}$ connectedness is convexity.

- $A_{m-1} \Longrightarrow B_{m}$ is proved below.

Choose an injective matrix $A \in \mathbb{Z}^{m \times(m-1)}$. Consider the action of an $(m-1)$ subtorus

$$
\begin{aligned}
\psi_{A}: \mathbb{T}^{m-1} & \longrightarrow \operatorname{Sympl}(M, \omega) \\
\theta & \longmapsto \psi_{A \theta} .
\end{aligned}
$$

Exercise. The action $\psi_{A}$ is hamiltonian with moment map $\mu_{A}=A^{t} \mu: M \rightarrow$ $\mathbb{R}^{m-1}$.

Given any $p_{0} \in \mu_{A}^{-1}(\xi)$,

$$
p \in \mu_{A}^{-1}(\xi) \Longleftrightarrow A^{t} \mu(p)=\xi=A^{t} \mu\left(p_{0}\right)
$$


so that

$$
\mu_{A}^{-1}(\xi)=\left\{p \in M \mid \mu(p)-\mu\left(p_{0}\right) \in \operatorname{ker} A^{t}\right\} .
$$

By the first part (statement $\left.A_{m-1}\right), \mu_{A}^{-1}(\xi)$ is connected. Therefore, if we connect $p_{0}$ to $p_{1}$ by a path $p_{t}$ in $\mu_{A}^{-1}(\xi)$, we obtain a path $\mu\left(p_{t}\right)-\mu\left(p_{0}\right)$ in ker $A^{t}$. But $\operatorname{ker} A^{t}$ is 1-dimensional. Hence, $\mu\left(p_{t}\right)$ must go through any convex combination of $\mu\left(p_{0}\right)$ and $\mu\left(p_{1}\right)$, which shows that any point on the line segment from $\mu\left(p_{0}\right)$ to $\mu\left(p_{1}\right)$ must be in $\mu(M)$ :

$$
(1-t) \mu\left(p_{0}\right)+t \mu\left(p_{1}\right) \in \mu(M), \quad 0 \leq t \leq 1 .
$$

Any $p_{0}, p_{1} \in M$ can be approximated arbitrarily closely by points $p_{0}^{\prime}$ and $p_{1}^{\prime}$ with $\mu\left(p_{1}^{\prime}\right)-\mu\left(p_{0}^{\prime}\right) \in \operatorname{ker} A^{t}$ for some injective matrix $A \in \mathbb{Z}^{m \times(m-1)}$. Taking limits $p_{0}^{\prime} \rightarrow p_{0}, p_{1}^{\prime} \rightarrow p_{1}$, we obtain that $\mu(M)$ is convex. ${ }^{15}$

To prove part 3, consider the fixed point set $C$ of $\psi$. Homework 21 shows that $C$ is a finite union of connected symplectic submanifolds, $C=C_{1} \cup \ldots \cup C_{N}$. The moment map is constant on each $C_{j}, \mu\left(C_{j}\right)=\eta_{j} \in \mathbb{R}^{m}, j=1, \ldots, N$. By the second part, the convex hull of $\left\{\eta_{1}, \ldots, \eta_{N}\right\}$ is contained in $\mu(M)$.

For the converse, suppose that $\xi \in \mathbb{R}^{m}$ and $\xi \notin$ convex hull of $\left\{\eta_{1}, \ldots, \eta_{N}\right\}$. Choose $X \in \mathbb{R}^{m}$ with rationally independent components and satisfying

$$
\langle\xi, X\rangle>\left\langle\eta_{j}, X\right\rangle \text {, for all } j \text {. }
$$

By the irrationality of $X$, the set $\{\exp t X(e) \mid t \in \mathbb{R}\}$ is dense in $\mathbb{T}^{m}$, hence the zeros of the vector field $X^{\#}$ on $M$ are the fixed points of the $\mathbb{T}^{m}$-action. Since $\mu^{X}=\langle\mu, X\rangle$ attains its maximum on one of the sets $C_{j}$, this implies

$$
\langle\xi, X\rangle>\sup _{p \in M}\langle\mu(p), X\rangle,
$$

hence $\xi \notin \mu(M)$. Therefore,

$$
\mu(M)=\text { convex hull of }\left\{\eta_{1}, \ldots, \eta_{N}\right\} .
$$

\subsection{Effective Actions}

An action of a group $G$ on a manifold $M$ is called effective if each group element $g \neq e$ moves at least one $p \in M$, that is,

$$
\bigcap_{p \in M} G_{p}=\{e\}
$$

where $G_{p}=\{g \in G \mid g \cdot p=p\}$ is the stabilizer of $p$.

\footnotetext{
${ }^{15}$ Clearly $\mu(M)$ is closed because it is compact.
} 
Corollary 27.2 Under the conditions of the convexity theorem, if the $\mathbb{T}^{m}$-action is effective, then there must be at least $m+1$ fixed points.

Proof. If the $\mathbb{T}^{m}$-action is effective, there must be a point $p$ where the moment map is a submersion, i.e., $\left(d \mu_{1}\right)_{p}, \ldots,\left(d \mu_{m}\right)_{p}$ are linearly independent. Hence, $\mu(p)$ is an interior point of $\mu(M)$, and $\mu(M)$ is a nondegenerate convex polytope. Any nondegenerate convex polytope in $\mathbb{R}^{m}$ must have at least $m+1$ vertices. The vertices of $\mu(M)$ are images of fixed points.

Theorem 27.3 Let $\left(M, \omega, \mathbb{T}^{m}, \mu\right)$ be a hamiltonian $\mathbb{T}^{m}$-space. If the $\mathbb{T}^{m}$-action is effective, then $\operatorname{dim} M \geq 2 m$.

Proof. On an orbit $\mathcal{O}$, the moment map $\mu(\mathcal{O})=\xi$ is constant. For $p \in \mathcal{O}$, the exterior derivative

$$
d \mu_{p}: T_{p} M \longrightarrow \mathfrak{g}^{*}
$$

$\operatorname{maps} T_{p} \mathcal{O}$ to 0. Thus

$$
T_{p} \mathcal{O} \subseteq \operatorname{ker} d \mu_{p}=\left(T_{p} \mathcal{O}\right)^{\omega}
$$

which shows that orbits $\mathcal{O}$ of a hamiltonian torus action are always isotropic submanifolds of $M$. In particular, $\operatorname{dim} \mathcal{O} \leq \frac{1}{2} \operatorname{dim} M$

Fact: If $\psi: \mathbb{T}^{m} \rightarrow \operatorname{Diff}(M)$ is an effective action, then it has orbits of dimension $m$; a proof may be found in [17].

Definition $27.4 A$ (symplectic) toric manifold ${ }^{16}$ is a compact connected symplectic manifold $(M, \omega)$ equipped with an effective hamiltonian action of a torus $\mathbb{T}$ of dimension equal to half the dimension of the manifold:

$$
\operatorname{dim} \mathbb{T}=\frac{1}{2} \operatorname{dim} M
$$

and with a choice of a corresponding moment map $\mu$.

Exercise. Show that an effective hamiltonian action of a torus $\mathbb{T}^{n}$ on a $2 n$ dimensional symplectic manifold gives rise to an integrable system.

Hint: The coordinates of the moment map are commuting integrals of motion.

\footnotetext{
${ }^{16}$ In these notes, a toric manifold is always a symplectic toric manifold.
} 


\subsection{Examples}

1. The circle $\mathbf{S}^{1}$ acts on the 2 -sphere $\left(S^{2}, \omega_{\text {standard }}=d \theta \wedge d h\right)$ by rotations with moment map $\mu=h$ equal to the height function and moment polytope $[-1,1]$.
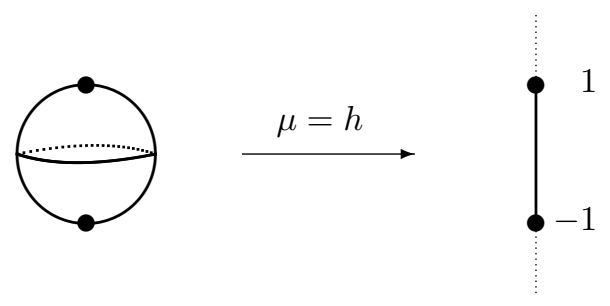

1.' The circle $\mathbf{S}^{1}$ acts on $\mathbb{C P}^{1}=\mathbb{C}^{2}-0 / \sim$ with the Fubini-Study form $\omega_{\mathrm{FS}}=$ $\frac{1}{4} \omega_{\text {standard }}$, by $e^{i \theta} \cdot\left[z_{0}, z_{1}\right]=\left[z_{0}, e^{i \theta} z_{1}\right]$. This is hamiltonian with moment map $\mu\left[z_{0}, z_{1}\right]=-\frac{1}{2} \cdot \frac{\left|z_{1}\right|^{2}}{\left|z_{0}\right|^{2}+\left|z_{1}\right|^{2}}$, and moment polytope $\left[-\frac{1}{2}, 0\right]$.

2. The $\mathbb{T}^{2}$-action on $\mathbb{C P}^{2}$ by

$$
\left(e^{i \theta_{1}}, e^{i \theta_{2}}\right) \cdot\left[z_{0}, z_{1}, z_{2}\right]=\left[z_{0}, e^{i \theta_{1}} z_{1}, e^{i \theta_{2}} z_{2}\right]
$$

has moment map

$$
\mu\left[z_{0}, z_{1}, z_{2}\right]=-\frac{1}{2}\left(\frac{\left|z_{1}\right|^{2}}{\left|z_{0}\right|^{2}+\left|z_{1}\right|^{2}+\left|z_{2}\right|^{2}}, \frac{\left|z_{2}\right|^{2}}{\left|z_{0}\right|^{2}+\left|z_{1}\right|^{2}+\left|z_{2}\right|^{2}}\right)
$$

The fixed points get mapped as

$$
\begin{aligned}
& {[1,0,0] \quad \longmapsto(0,0)} \\
& {[0,1,0] \quad \longmapsto\left(-\frac{1}{2}, 0\right)} \\
& {[0,0,1] \longmapsto\left(0,-\frac{1}{2}\right) .}
\end{aligned}
$$

Notice that the stabilizer of a preimage of the edges is $S^{1}$, while the action is free at preimages of interior points of the moment polytope. 


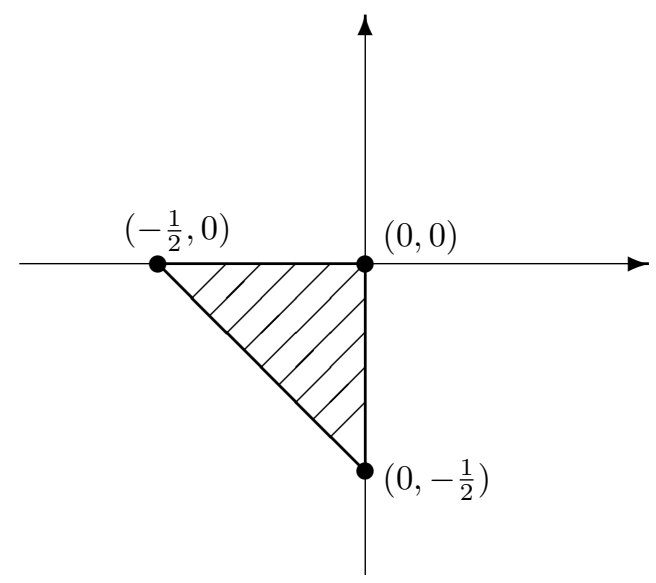

Exercise. What is the moment polytope for the $\mathbb{T}^{3}$-action on $\mathbb{C P}^{3}$ as

$$
\left(e^{i \theta_{1}}, e^{i \theta_{2}}, e^{i \theta_{3}}\right) \cdot\left[z_{0}, z_{1}, z_{2}, z_{3}\right]=\left[z_{0}, e^{i \theta_{1}} z_{1}, e^{i \theta_{2}} z_{2}, e^{i \theta_{3}} z_{3}\right] ?
$$

Exercise. What is the moment polytope for the $\mathbb{T}^{2}$-action on $\mathbb{C P}^{1} \times \mathbb{C P}^{1}$ as

$$
\left(e^{i \theta}, e^{i \eta}\right) \cdot\left(\left[z_{0}, z_{1}\right],\left[w_{0}, w_{1}\right]\right)=\left(\left[z_{0}, e^{i \theta} z_{1}\right],\left[w_{0}, e^{i \eta} w_{1}\right]\right) ?
$$




\section{Homework 21: Connectedness}

Consider a hamiltonian action $\psi: \mathbb{T}^{m} \rightarrow \operatorname{Sympl}(M, \omega), \theta \mapsto \psi_{\theta}$, of an $m$ dimensional torus on a $2 n$-dimensional compact connected symplectic manifold $(M, \omega)$. If we identify the Lie algebra of $\mathbb{T}^{m}$ with $\mathbb{R}^{m}$ by viewing $\mathbb{T}^{m}=\mathbb{R}^{m} / \mathbb{Z}^{m}$, and we identify the Lie algebra with its dual via the standard inner product, then the moment map for $\psi$ is $\mu: M \rightarrow \mathbb{R}^{m}$.

1. Show that there exists a compatible almost complex structure $J$ on $(M, \omega)$ which is invariant under the $\mathbb{T}^{m}$-action, that is, $\psi_{\theta}^{*} J=J \psi_{\theta}^{*}$, for all $\theta \in \mathbb{T}^{m}$.

Hint: We cannot average almost complex structures, but we can average riemannian metrics (why?). Given a riemannian metric $g_{0}$ on $M$, its $\mathbb{T}^{m}$-average $g=\int_{\mathbb{T}^{m}} \psi_{\theta}^{*} g_{0} d \theta$ is $\mathbb{T}^{m}$-invariant.

2. Show that, for any subgroup $G \subseteq \mathbb{T}^{m}$, the fixed-point set for $G$,

$$
\operatorname{Fix}(G)=\bigcap_{\theta \in G} \operatorname{Fix}\left(\psi_{\theta}\right),
$$

is a symplectic submanifold of $M$.

Hint: For each $p \in \operatorname{Fix}(G)$ and each $\theta \in G$, the differential of $\psi_{\theta}$ at $p$,

$$
d \psi_{\theta}(p): T_{p} M \longrightarrow T_{p} M,
$$

preserves the complex structure $J_{p}$ on $T_{p} M$. Consider the exponential map $\exp _{p}$ : $T_{p} M \rightarrow M$ with respect to the invariant riemannian metric $g(\cdot, \cdot)=\omega(\cdot, J \cdot)$. Show that, by uniqueness of geodesics, $\exp _{p}$ is equivariant, i.e.,

$$
\exp _{p}\left(d \psi_{\theta}(p) v\right)=\psi_{\theta}\left(\exp _{p} v\right)
$$

for any $\theta \in G, v \in T_{p} M$. Conclude that the fixed points of $\psi_{\theta}$ near $p$ correspond to the fixed points of $d \psi_{\theta}(p)$ on $T_{p} M$, that is

$$
T_{p} \text { Fix }(G)=\bigcap_{\theta \in G} \operatorname{ker}\left(\operatorname{Id}-d \psi_{\theta}(p)\right) .
$$

Since $d \psi_{\theta}(p) \circ J_{p}=J_{p} \circ d \psi_{\theta}(p)$, the eigenspace with eigenvalue 1 is invariant under $J_{p}$, and is therefore a symplectic subspace.

3. A smooth function $f: M \rightarrow \mathbb{R}$ on a compact riemannian manifold $M$ is called a Morse-Bott function if its critical set Crit $(f)=\{p \in M \mid d f(p)=0\}$ is a submanifold of $M$ and for every $p \in \operatorname{Crit}(f), T_{p}$ Crit $(f)=\operatorname{ker} \nabla^{2} f(p)$ where $\nabla^{2} f(p): T_{p} M \rightarrow T_{p} M$ denotes the linear operator obtained from the hessian via the riemannian metric. This is the natural generalization of the notion of Morse function to the case where the critical set is not just isolated points. If $f$ is a Morse-Bott function, then Crit $(f)$ decomposes into finitely many connected critical manifolds $C$. The tangent space $T_{p} M$ at $p \in C$ decomposes as a direct sum

$$
T_{p} M=T_{p} C \oplus E_{p}^{+} \oplus E_{p}^{-}
$$

where $E_{p}^{+}$and $E_{p}^{-}$are spanned by the positive and negative eigenspaces of $\nabla^{2} f(p)$. The index of a connected critical submanifold $C$ is $n_{C}^{-}=\operatorname{dim} E_{p}^{-}$, for any $p \in C$, whereas the coindex of $C$ is $n_{C}^{+}=\operatorname{dim} E_{p}^{+}$. 
For each $X \in \mathbb{R}^{m}$, let $\mu^{X}=\langle\mu, X\rangle: M \rightarrow \mathbb{R}$ be the component of $\mu$ along $X$. Show that $\mu^{X}$ is a Morse-Bott function with even-dimensional critical manifolds of even index. Moreover, show that the critical set

$$
\operatorname{Crit}\left(\mu^{X}\right)=\bigcap_{\theta \in \mathbb{T}^{X}} \operatorname{Fix}\left(\psi_{\theta}\right)
$$

is a symplectic manifold, where $\mathbb{T}^{X}$ is the closure of the subgroup of $\mathbb{T}^{m}$ generated by $X$.

Hint: Assume first that $X$ has components independent over $\mathbb{Q}$, so that $\mathbb{T}^{X}=\mathbb{T}^{m}$ and Crit $\left(\mu^{X}\right)=$ Fix $\left(\mathbb{T}^{m}\right)$. Apply exercise 2. To prove that $T_{p}$ Crit $\left(\mu^{X}\right)=$ $\operatorname{ker} \nabla^{2} \mu^{X}(p)$, show that $\operatorname{ker} \nabla^{2} \mu^{X}(p)=\cap_{\theta \in \mathbb{T}^{m}} \operatorname{ker}\left(\operatorname{Id}-d \psi_{\theta}(p)\right)$. To see this, notice that the 1-parameter group of matrices $\left(d \psi_{\exp t X}\right)_{p}$ coincides with $\exp \left(t v_{p}\right)$, where $v_{p}=-J_{p} \nabla^{2} \mu^{X}(p): T_{p} M \rightarrow T_{p} M$ is a vector field on $T_{p} M$. The kernel of $\nabla^{2} \mu^{X}(p)$ corresponds to the fixed points of $d \psi_{t X}(p)$, and since $X$ has rationally independent components, these are the common fixed points of all $d \psi_{\theta}(p), \theta \in$ $\mathbb{T}^{m}$. The eigenspaces of $\nabla^{2} \mu^{X}(p)$ are even-dimensional because they are invariant under $J_{p}$.

4. The moment map $\mu=\left(\mu_{1}, \ldots, \mu_{m}\right)$ is called effective if the 1 -forms $d \mu_{1}, \ldots, d \mu_{m}$ of its components are linearly independent. Show that, if $\mu$ is not effective, then the action reduces to that of an $(m-1)$-subtorus.

Hint: If $\mu$ is not effective, then the function $\mu^{X}=\langle\mu, X\rangle$ is constant for some nonzero $X \in \mathbb{R}^{m}$. Show that we can neglect the direction of $X$.

5. Prove that the level set $\mu^{-1}(\xi)$ is connected for every regular value $\xi \in \mathbb{R}^{m}$.

Hint: Prove by induction over $m=\operatorname{dim} \mathbb{T}^{m}$. For the case $m=1$, use the lemma that all level sets $f^{-1}(c)$ of a Morse-Bott function $f: M \rightarrow \mathbb{R}$ on a compact manifold $M$ are necessarily connected, if the critical manifolds all have index and coindex $\neq 1$ (see $[83$, p.178-179]). For the induction step, you can assume that $\psi$ is effective. Then, for every $0 \neq X \in \mathbb{R}^{m}$, the function $\mu^{X}: M \rightarrow \mathbb{R}$ is not constant. Show that $\mathcal{C}:=\cup_{X \neq 0}$ Crit $\mu^{X}=\cup_{0 \neq X \in \mathbb{Z}^{m}}$ Crit $\mu^{X}$ where each Crit $\mu^{X}$ is an even-dimensional proper submanifold, so the complement $M \backslash \mathcal{C}$ must be dense in $M$. Show that $M \backslash \mathcal{C}$ is open. Hence, by continuity, to show that $\mu^{-1}(\xi)$ is connected for every regular value $\xi=\left(\xi_{1}, \ldots, \xi_{m}\right) \in \mathbb{R}^{m}$, it suffices to show that $\mu^{-1}(\xi)$ is connected whenever $\left(\xi_{1}, \ldots, \xi_{m-1}\right)$ is a regular value for a reduced moment map $\left(\mu_{1}, \ldots, \mu_{m-1}\right)$. By the induction hypothesis, the manifold $Q=\cap_{j=1}^{m-1} \mu_{j}^{-1}\left(\xi_{j}\right)$ is connected whenever $\left(\xi_{1}, \ldots, \xi_{m-1}\right)$ is a regular value for $\left(\mu_{1}, \ldots, \mu_{m-1}\right)$. It suffices to show that the function $\mu_{m}: Q \rightarrow \mathbb{R}$ has only critical manifolds of even index and coindex (see [83, p.183]), because then, by the lemma, the level sets $\mu^{-1}(\xi)=Q \cap \mu_{m}^{-1}\left(\xi_{m}\right)$ are connected for every $\xi_{m}$. 



\section{Part XI}

\section{Symplectic Toric Manifolds}

Native to algebraic geometry, toric manifolds have been studied by symplectic geometers as examples of extremely symmetric hamiltonian spaces, and as guinea pigs for new theorems. Delzant showed that symplectic toric manifolds are classified (as hamiltonian spaces) by a set of special polytopes.

\section{Classification of Symplectic Toric Manifolds}

\subsection{Delzant Polytopes}

A $2 n$-dimensional (symplectic) toric manifold is a compact connected symplectic manifold $\left(M^{2 n}, \omega\right)$ equipped with an effective hamiltonian action of an $n$-torus $\mathbb{T}^{n}$ and with a corresponding moment map $\mu: M \rightarrow \mathbb{R}^{n}$.

Definition 28.1 $A$ Delzant polytope $\Delta$ in $\mathbb{R}^{n}$ is a convex polytope satisfying:

- it is simple, i.e., there are $n$ edges meeting at each vertex;

- it is rational, i.e., the edges meeting at the vertex $p$ are rational in the sense that each edge is of the form $p+t u_{i}, t \geq 0$, where $u_{i} \in \mathbb{Z}^{n}$;

- it is smooth, i.e., for each vertex, the corresponding $u_{1}, \ldots, u_{n}$ can be chosen to be a $\mathbb{Z}$-basis of $\mathbb{Z}^{n}$.

Remark. The Delzant polytopes are the simple rational smooth polytopes. These are closely related to the Newton polytopes (which are the nonsingular $n$-valent polytopes), except that the vertices of a Newton polytope are required to lie on the integer lattice and for a Delzant polytope they are not.

\section{Examples of Delzant polytopes:}
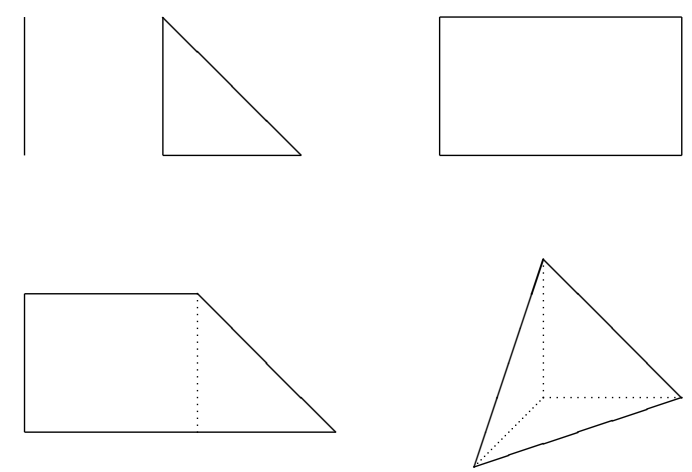
The dotted vertical line in the trapezoidal example means nothing, except that it is a picture of a rectangle plus an isosceles triangle. For "taller" triangles, smoothness would be violated. "Wider" triangles (with integral slope) may still be Delzant. The family of the Delzant trapezoids of this type, starting with the rectangle, correspond, under the Delzant construction, to Hirzebruch surfaces; see Homework 22.

Examples of polytopes which are not Delzant:
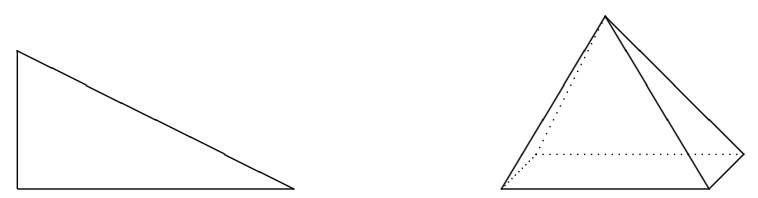

The picture on the left fails the smoothness condition, whereas the picture on the right fails the simplicity condition.

\section{Algebraic description of Delzant polytopes:}

A facet of a polytope is a $(n-1)$-dimensional face.

Let $\Delta$ be a Delzant polytope with $n=\operatorname{dim} \Delta$ and $d=$ number of facets.

A lattice vector $v \in \mathbb{Z}^{n}$ is primitive if it cannot be written as $v=k u$ with $u \in \mathbb{Z}^{n}, k \in \mathbb{Z}$ and $|k|>1$; for instance, $(1,1),(4,3),(1,0)$ are primitive, but $(2,2),(3,6)$ are not.

Let $v_{i} \in \mathbb{Z}^{n}, i=1, \ldots, d$, be the primitive outward-pointing normal vectors to the facets.

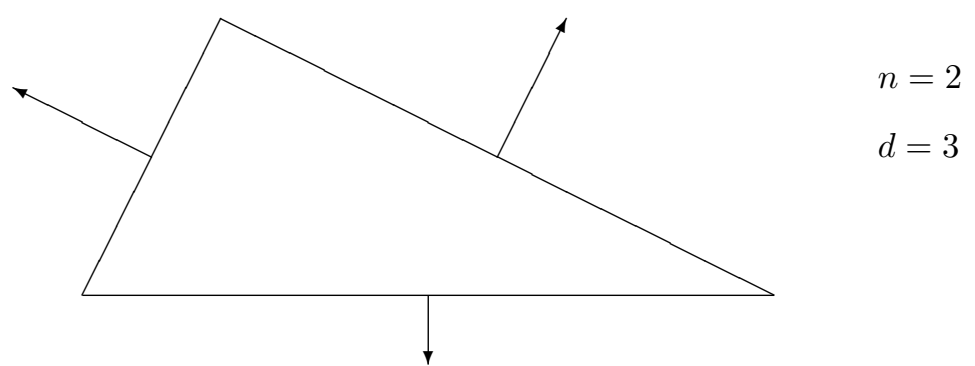

Then we can describe $\Delta$ as an intersection of halfspaces

$$
\Delta=\left\{x \in\left(\mathbb{R}^{n}\right)^{*} \mid\left\langle x, v_{i}\right\rangle \leq \lambda_{i}, i=1, \ldots, d\right\} \quad \text { for some } \lambda_{i} \in \mathbb{R} .
$$


Example. For the picture below, we have

$$
\begin{aligned}
\Delta & =\left\{x \in\left(\mathbb{R}^{2}\right)^{*} \mid x_{1} \geq 0, x_{2} \geq 0, x_{1}+x_{2} \leq 1\right\} \\
& =\left\{x \in\left(\mathbb{R}^{2}\right)^{*} \mid\langle x,(-1,0)\rangle \leq 0,\langle x,(0,-1)\rangle \leq 0,\langle x,(1,1)\rangle \leq 1\right\}
\end{aligned}
$$

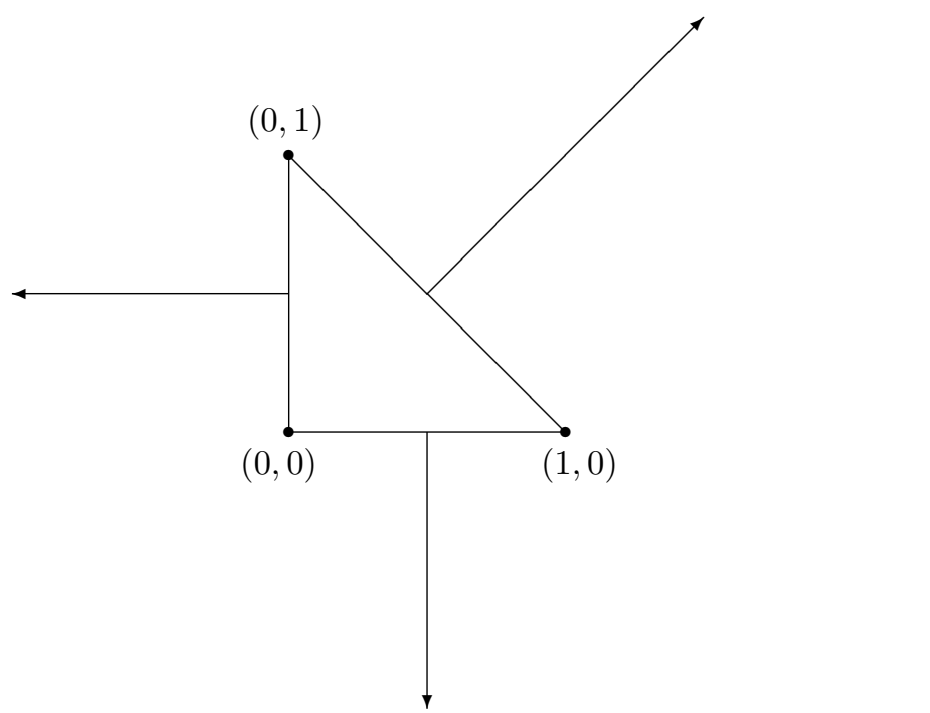

\subsection{Delzant Theorem}

We do not have a classification of symplectic manifolds, but we do have a classification of toric manifolds in terms of combinatorial data. This is the content of the Delzant theorem.

Theorem 28.2 (Delzant [23]) Toric manifolds are classified by Delzant polytopes. More specifically, there is the following one-to-one correspondence

$$
\begin{aligned}
\{\text { toric manifolds }\} & \stackrel{1-1}{\longmapsto}\{\text { Delzant polytopes }\} \\
\left(M^{2 n}, \omega, \mathbb{T}^{n}, \mu\right) & \longmapsto \mu(M) .
\end{aligned}
$$

We will prove the existence part (or surjectivity) in the Delzant theorem following [54]. Given a Delzant polytope, what is the corresponding toric manifold?

$$
\left(M_{\Delta}, \omega_{\Delta}, \mathbb{T}^{n}, \mu\right) \stackrel{?}{\longleftarrow} \Delta^{n}
$$




\subsection{Sketch of Delzant Construction}

Let $\Delta$ be a Delzant polytope with $d$ facets. Let $v_{i} \in \mathbb{Z}^{n}, i=1, \ldots, d$, be the primitive outward-pointing normal vectors to the facets. For some $\lambda_{i} \in \mathbb{R}$,

$$
\Delta=\left\{x \in\left(\mathbb{R}^{n}\right)^{*} \mid\left\langle x, v_{i}\right\rangle \leq \lambda_{i}, i=1, \ldots, d\right\}
$$

Let $e_{1}=(1,0, \ldots, 0), \ldots, e_{d}=(0, \ldots, 0,1)$ be the standard basis of $\mathbb{R}^{d}$. Consider

$$
\begin{aligned}
\pi: \mathbb{R}^{d} & \longrightarrow \mathbb{R}^{n} \\
e_{i} & \longmapsto v_{i} .
\end{aligned}
$$

Claim. The map $\pi$ is onto and maps $\mathbb{Z}^{d}$ onto $\mathbb{Z}^{n}$.

Proof. The set $\left\{e_{1}, \ldots, e_{d}\right\}$ is a basis of $\mathbb{Z}^{d}$. The set $\left\{v_{1}, \ldots, v_{d}\right\}$ spans $\mathbb{Z}^{n}$ for the following reason. At a vertex $p$, the edge vectors $u_{1}, \ldots, u_{n} \in\left(\mathbb{R}^{n}\right)^{*}$, form a basis for $\left(\mathbb{Z}^{n}\right)^{*}$ which, without loss of generality, we may assume is the standard basis. Then the corresponding primitive normal vectors to the facets meeting at $p$ are symmetric (in the sense of multiplication by -1 ) to the $u_{i}$ 's, hence form a basis of $\mathbb{Z}^{n}$.

Therefore, $\pi$ induces a surjective map, still called $\pi$, between tori:

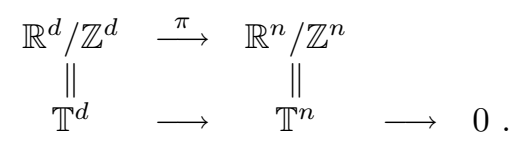

Let

$$
\begin{aligned}
N & =\text { kernel of } \pi\left(N \text { is a Lie subgroup of } \mathbb{T}^{d}\right) \\
\mathfrak{n} & =\text { Lie algebra of } N \\
\mathbb{R}^{d} & =\text { Lie algebra of } \mathbb{T}^{d} \\
\mathbb{R}^{n} & =\text { Lie algebra of } \mathbb{T}^{n} .
\end{aligned}
$$

The exact sequence of tori

$$
0 \longrightarrow N \stackrel{i}{\longrightarrow} \mathbb{T}^{d} \stackrel{\pi}{\longrightarrow} \mathbb{T}^{n} \longrightarrow 0
$$

induces an exact sequence of Lie algebras

$$
0 \longrightarrow \mathfrak{n} \stackrel{i}{\longrightarrow} \mathbb{R}^{d} \stackrel{\pi}{\longrightarrow} \mathbb{R}^{n} \longrightarrow 0
$$

with dual exact sequence

$$
0 \longrightarrow\left(\mathbb{R}^{n}\right)^{*} \stackrel{\pi^{*}}{\longrightarrow}\left(\mathbb{R}^{d}\right)^{*} \stackrel{i^{*}}{\longrightarrow} \mathfrak{n}^{*} \longrightarrow 0
$$

Now consider $\mathbb{C}^{d}$ with symplectic form $\omega_{0}=\frac{i}{2} \sum d z_{k} \wedge d \bar{z}_{k}$, and standard hamiltonian action of $\mathbb{T}^{d}$

$$
\left(e^{2 \pi i t_{1}}, \ldots, e^{2 \pi i t_{d}}\right) \cdot\left(z_{1}, \ldots, z_{d}\right)=\left(e^{2 \pi i t_{1}} z_{1}, \ldots, e^{2 \pi i t_{d}} z_{d}\right)
$$


The moment map is $\phi: \mathbb{C}^{d} \longrightarrow\left(\mathbb{R}^{d}\right)^{*}$

$$
\phi\left(z_{1}, \ldots, z_{d}\right)=-\pi\left(\left|z_{1}\right|^{2}, \ldots,\left|z_{d}\right|^{2}\right)+\text { constant },
$$

where we choose the constant to be $\left(\lambda_{1}, \ldots, \lambda_{d}\right)$. What is the moment map for the action restricted to the subgroup $N$ ?

Exercise. Let $G$ be any compact Lie group and $H$ a closed subgroup of $G$, with $\mathfrak{g}$ and $\mathfrak{h}$ the respective Lie algebras. The inclusion $i: \mathfrak{h} \hookrightarrow \mathfrak{g}$ is dual to the projection $i^{*}: \mathfrak{g}^{*} \rightarrow \mathfrak{h}^{*}$. Suppose that $(M, \omega, G, \phi)$ is a hamiltonian $G$-space. Show that the restriction of the $G$-action to $H$ is hamiltonian with moment map

$$
i^{*} \circ \phi: M \longrightarrow \mathfrak{h}^{*} \text {. }
$$

The subtorus $N$ acts on $\mathbb{C}^{d}$ in a hamiltonian way with moment map

$$
i^{*} \circ \phi: \mathbb{C}^{d} \longrightarrow \mathfrak{n}^{*} \text {. }
$$

Let $Z=\left(i^{*} \circ \phi\right)^{-1}(0)$ be the zero-level set.

Claim. The set $Z$ is compact and $N$ acts freely on $Z$.

This claim will be proved in the next lecture.

By the first claim, $0 \in \mathfrak{n}^{*}$ is a regular value of $i^{*} \circ \phi$. Hence, $Z$ is a compact submanifold of $\mathbb{C}^{d}$ of dimension

$$
\operatorname{dim}_{\mathbb{R}} Z=2 d-\underbrace{(d-n)}_{\operatorname{dim} \mathfrak{n}^{*}}=d+n .
$$

The orbit space $M_{\Delta}=Z / N$ is a compact manifold of dimension

$$
\operatorname{dim}_{\mathbb{R}} M_{\Delta}=d+n-\underbrace{(d-n)}_{\operatorname{dim} N}=2 n .
$$

The point-orbit map $p: Z \rightarrow M_{\Delta}$ is a principal $N$-bundle over $M_{\Delta}$.

Consider the diagram

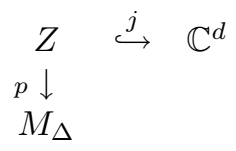

where $j: Z \hookrightarrow \mathbb{C}^{d}$ is inclusion. The Marsden-Weinstein-Meyer theorem guarantees the existence of a symplectic form $\omega_{\Delta}$ on $M_{\Delta}$ satisfying

$$
p^{*} \omega_{\Delta}=j^{*} \omega_{0} \text {. }
$$

Exercise. Work out all details in the following simple example.

Let $\Delta=[0, a] \subset \mathbb{R}^{*}(n=1, d=2)$. Let $v(=1)$ be the standard basis vector in $\mathbb{R}$. Then

$$
\begin{array}{rll}
\Delta: & \left\langle x, v_{1}\right\rangle \leq 0 & v_{1}=-v \\
\left\langle x, v_{2}\right\rangle \leq a & v_{2}=v .
\end{array}
$$


The projection

$$
\begin{aligned}
& \mathbb{R}^{2} \stackrel{\pi}{\longrightarrow} \mathbb{R} \\
& e_{1} \longmapsto-v \\
& e_{2} \longmapsto v
\end{aligned}
$$

has kernel equal to the span of $\left(e_{1}+e_{2}\right)$, so that $N$ is the diagonal subgroup of $\mathbb{T}^{2}=S^{1} \times S^{1}$. The exact sequences become

$$
\begin{aligned}
& 0 \longrightarrow N \stackrel{i}{\longrightarrow} \mathbb{T}^{2} \stackrel{\pi}{\longrightarrow} \quad S^{1} \quad \longrightarrow 0 \\
& 0 \longrightarrow \mathbb{R}^{*} \stackrel{\pi^{*}}{\longrightarrow}\left(\mathbb{R}^{2}\right)^{*} \stackrel{i^{*}}{\longrightarrow} \quad \mathfrak{n}^{*} \quad \longrightarrow 0 \\
& \left(x_{1}, x_{2}\right) \longmapsto x_{1}+x_{2} \text {. }
\end{aligned}
$$

The action of the diagonal subgroup $N=\left\{\left(e^{2 \pi i t}, e^{2 \pi i t}\right) \in S^{1} \times S^{1}\right\}$ on $\mathbb{C}^{2}$,

$$
\left(e^{2 \pi i t}, e^{2 \pi i t}\right) \cdot\left(z_{1}, z_{2}\right)=\left(e^{2 \pi i t} z_{1}, e^{2 \pi i t} z_{2}\right),
$$

has moment map

$$
\left(i^{*} \circ \phi\right)\left(z_{1}, z_{2}\right)=-\pi\left(\left|z_{1}\right|^{2}+\left|z_{2}\right|^{2}\right)+a,
$$

with zero-level set

$$
\left(i^{*} \circ \phi\right)^{-1}(0)=\left\{\left(z_{1}, z_{2}\right) \in \mathbb{C}^{2}:\left|z_{1}\right|^{2}+\left|z_{2}\right|^{2}=\frac{a}{\pi}\right\} .
$$

Hence, the reduced space is

$$
\left(i^{*} \circ \phi\right)^{-1}(0) / N=\mathbb{C P}^{1} \quad \text { projective space! }
$$




\section{Delzant Construction}

\subsection{Algebraic Set-Up}

Let $\Delta$ be a Delzant polytope with $d$ facets. We can write $\Delta$ as

$$
\Delta=\left\{x \in\left(\mathbb{R}^{n}\right)^{*} \mid\left\langle x, v_{i}\right\rangle \leq \lambda_{i}, i=1, \ldots, d\right\},
$$

for some $\lambda_{i} \in \mathbb{R}$. Recall the exact sequences from the previous lecture

$$
\begin{aligned}
& 0 \longrightarrow N \stackrel{i}{\longrightarrow} \mathbb{T}^{d} \stackrel{\pi}{\longrightarrow} \mathbb{T}^{n} \longrightarrow 0 \\
& 0 \longrightarrow \mathfrak{n} \stackrel{i}{\longrightarrow} \mathbb{R}^{d} \stackrel{\pi}{\longrightarrow} \mathbb{R}^{n} \longrightarrow 0 \\
& e_{i} \longmapsto v_{i}
\end{aligned}
$$

and the dual sequence

$$
0 \longrightarrow\left(\mathbb{R}^{n}\right)^{*} \stackrel{\pi^{*}}{\longrightarrow}\left(\mathbb{R}^{d}\right)^{*} \stackrel{i^{*}}{\longrightarrow} \mathfrak{n}^{*} \longrightarrow 0
$$

The standard hamiltonian action of $\mathbb{T}^{d}$ on $\mathbb{C}^{d}$

$$
\left(e^{2 \pi i t_{1}}, \ldots, e^{2 \pi i t_{d}}\right) \cdot\left(z_{1}, \ldots, z_{d}\right)=\left(e^{2 \pi i t_{1}} z_{1}, \ldots, e^{2 \pi i t_{d}} z_{d}\right)
$$

has moment map $\phi: \mathbb{C}^{d} \rightarrow\left(\mathbb{R}^{d}\right)^{*}$ given by

$$
\phi\left(z_{1}, \ldots, z_{d}\right)=-\pi\left(\left|z_{1}\right|^{2}, \ldots,\left|z_{d}\right|^{2}\right)+\left(\lambda_{1}, \ldots, \lambda_{d}\right) .
$$

The restriction of this action to $N$ has moment map

$$
i^{*} \circ \phi: \mathbb{C}^{d} \longrightarrow \mathfrak{n}^{*} \text {. }
$$

\subsection{The Zero-Level}

Let $Z=\left(i^{*} \circ \phi\right)^{-1}(0)$.

Proposition 29.1 The level $Z$ is compact and $N$ acts freely on $Z$.

Proof. Let $\Delta^{\prime}$ be the image of $\Delta$ by $\pi^{*}$. We will show that $\phi(Z)=\Delta^{\prime}$. Since $\phi$ is a proper map and $\Delta^{\prime}$ is compact, it will follow that $Z$ is compact.

Lemma 29.2 Let $y \in\left(\mathbb{R}^{d}\right)^{*}$. Then:

$$
y \in \Delta^{\prime} \Longleftrightarrow y \text { is in the image of } Z \text { by } \phi .
$$

Proof of the lemma. The value $y$ is in the image of $Z$ by $\phi$ if and only if both of the following conditions hold:

1. $y$ is in the image of $\phi$; 
2. $i^{*} y=0$.

Using the expression for $\phi$ and the third exact sequence, we see that these conditions are equivalent to:

1. $\left\langle y, e_{i}\right\rangle \leq \lambda_{i}$ for $i=1, \ldots, d$.

2. $y=\pi^{*}(x)$ for some $x \in\left(\mathbb{R}^{n}\right)^{*}$.

Suppose that the second condition holds, so that $y=\pi^{*}(x)$. Then

$$
\begin{aligned}
\left\langle y, e_{i}\right\rangle \leq \lambda_{i}, \forall i & \Longleftrightarrow\left\langle\pi^{*}(x), e_{i}\right\rangle \leq \lambda_{i}, \forall i \\
& \Longleftrightarrow\left\langle x, \pi\left(e_{i}\right)\right\rangle \leq \lambda_{i}, \forall i \\
& \Longleftrightarrow x \in \Delta .
\end{aligned}
$$

Thus, $y \in \phi(Z) \Longleftrightarrow y \in \pi^{*}(\Delta)=\Delta^{\prime}$.

Hence, we have a surjective proper map $\phi: Z \rightarrow \Delta^{\prime}$. Since $\Delta^{\prime}$ is compact, we conclude that $Z$ is compact. It remains to show that $N$ acts freely on $Z$.

We define a stratification of $Z$ with three equivalent descriptions:

- Define a stratification on $\Delta^{\prime}$ whose $i$ th stratum is the closure of the union of the $i$-dimensional faces of $\Delta^{\prime}$. Pull this stratification back to $Z$ by $\phi$.

We can obtain a more explicit description of the stratification on $Z$ :

- Let $F$ be a face of $\Delta^{\prime}$ with $\operatorname{dim} F=n-r$. Then $F$ is characterized (as a subset of $\Delta^{\prime}$ ) by $r$ equations

$$
\left\langle y, e_{i}\right\rangle=\lambda_{i}, \quad i=i_{1}, \ldots, i_{r} .
$$

We write $F=F_{I}$ where $I=\left(i_{1}, \ldots, i_{r}\right)$ has $1 \leq i_{1}<i_{2} \ldots<i_{r} \leq d$.

Let $z=\left(z_{1}, \ldots, z_{d}\right) \in Z$.

$$
\begin{aligned}
z \in \phi^{-1}\left(F_{I}\right) & \Longleftrightarrow \phi(z) \in F_{I} \\
& \Longleftrightarrow\left\langle\phi(z), e_{i}\right\rangle=\lambda_{i}, \quad \forall i \in I \\
& \Longleftrightarrow-\pi\left|z_{i}\right|^{2}+\lambda_{i}=\lambda_{i}, \quad \forall i \in I \\
& \Longleftrightarrow z_{i}=0, \quad \forall i \in I .
\end{aligned}
$$

- The $\mathbb{T}^{d}$-action on $\mathbb{C}^{d}$ preserves $\phi$, so the $\mathbb{T}^{d}$-action takes $Z=\phi^{-1}\left(\Delta^{\prime}\right)$ onto itself, so $\mathbb{T}^{d}$ acts on $Z$.

Exercise. The stratification of $Z$ is just the stratification of $Z$ into $\mathbb{T}^{d}$ orbit types. More specifically, if $z \in Z$ and $\phi(z) \in F_{I}$ then the stabilizer of $z$ in $\mathbb{T}^{d}$ is $\left(\mathbb{T}^{d}\right)_{I}$ where

$$
\begin{gathered}
I=\left(i_{1}, \ldots, i_{r}\right), \\
F_{I}=\left\{y \in \Delta^{\prime} \mid\left\langle y, e_{i}\right\rangle=\lambda_{i}, \forall i \in I\right\},
\end{gathered}
$$

and

$$
\left(\mathbb{T}^{d}\right)_{I}=\left\{\left(e^{2 \pi i t_{1}}, \ldots, e^{2 \pi i t_{d}}\right) \mid e^{2 \pi i t_{s}}=1, \forall s \notin I\right\}
$$




$$
\begin{aligned}
& \text { Hint: Suppose that } z=\left(z_{1}, \ldots, z_{d}\right) \in \mathbb{C}^{d} \text {. Then } \\
& \qquad\left(e^{2 \pi i t_{1}} z_{1}, \ldots, e^{2 \pi i t_{d}} z_{d}\right)=\left(z_{1}, \ldots, z_{d}\right) \\
& \text { if and only if } e^{2 \pi i t_{s}}=1 \text { whenever } z_{s} \neq 0 .
\end{aligned}
$$

In order to show that $N$ acts freely on $Z$, consider the worst case scenario of points $z \in Z$ whose stabilizer under the action of $\mathbb{T}^{d}$ is a large as possible. Now $\left(\mathbb{T}^{d}\right)_{I}$ is largest when $F_{I}=\{y\}$ is a vertex of $\Delta^{\prime}$. Then $y$ satisfies $n$ equations

$$
\left\langle y, e_{i}\right\rangle=\lambda_{i}, \quad i \in I=\left\{i_{1}, \ldots, i_{n}\right\} .
$$

Lemma 29.3 Let $z \in Z$ be such that $\phi(z)$ is a vertex of $\Delta^{\prime}$. Let $\left(\mathbb{T}^{d}\right)_{I}$ be the stabilizer of $z$. Then the map $\pi: \mathbb{T}^{d} \rightarrow \mathbb{T}^{n}$ maps $\left(\mathbb{T}^{d}\right)_{I}$ bijectively onto $\mathbb{T}^{n}$.

Since $N=\operatorname{ker} \pi$, this lemma shows that in the worst case, the stabilizer of $z$ intersects $N$ in the trivial group. It will follow that $N$ acts freely at this point and hence on $Z$.

Proof of the lemma. Suppose that $\phi(z)=y$ is a vertex of $\Delta^{\prime}$. Renumber the indices so that

$$
I=(1,2, \ldots, n)
$$

Then

$$
\left(\mathbb{T}^{d}\right)_{I}=\left\{\left(e^{2 \pi i t_{1}}, \ldots, e^{2 \pi i t_{n}}, 1, \ldots, 1\right) \mid t_{i} \in \mathbb{R}\right\}
$$

The hyperplanes meeting at $y$ are

$$
\left\langle y^{\prime}, e_{i}\right\rangle=\lambda_{i}, \quad i=1, \ldots, n
$$

By definition of Delzant polytope, the set $\pi\left(e_{1}\right), \ldots, \pi\left(e_{n}\right)$ is a basis of $\mathbb{Z}^{n}$. Thus, $\pi:\left(\mathbb{T}^{d}\right)_{I} \rightarrow \mathbb{T}^{n}$ is bijective.

This proves the theorem in the worst case scenario, and hence in general.

\subsection{Conclusion of the Delzant Construction}

We continue the construction of $\left(M_{\Delta}, \omega_{\Delta}\right)$ from $\Delta$. We already have that

$$
M_{\Delta}=Z / N
$$

is a compact $2 n$-dimensional manifold. Let $\omega_{\Delta}$ be the reduced symplectic form.

Claim. The manifold $\left(M_{\Delta}, \omega_{\Delta}\right)$ is a hamiltonian $\mathbb{T}^{n}$-space with a moment map $\mu$ having image $\mu\left(M_{\Delta}\right)=\Delta$.

Suppose that $z \in Z$. The stabilizer of $z$ with respect to the $\mathbb{T}^{d}$-action is $\left(\mathbb{T}^{d}\right)_{I}$, and

$$
\left(\mathbb{T}^{d}\right)_{I} \cap N=\{e\}
$$


In the worst case scenario, $F_{I}$ is a vertex of $\Delta^{\prime}$ and $\left(\mathbb{T}^{d}\right)_{I}$ is an $n$-dimensional subgroup of $\mathbb{T}^{d}$. In any case, there is a right inverse map $\pi^{-1}: \mathbb{T}^{n} \rightarrow\left(\mathbb{T}^{d}\right)_{I}$. Thus, the exact sequence

$$
0 \longrightarrow N \longrightarrow \mathbb{T}^{d} \longrightarrow \mathbb{T}^{n} \longrightarrow 0
$$

splits, and $\mathbb{T}^{d}=N \times \mathbb{T}^{n}$.

Apply the results on reduction for product groups (Section 24.3) to our situation of $\mathbb{T}^{d}=N \times \mathbb{T}^{n}$ acting on $\left(M_{\Delta}, \omega_{\Delta}\right)$. The moment map is

$$
\phi: \mathbb{C}^{d} \longrightarrow\left(\mathbb{R}^{d}\right)^{*}=\mathfrak{n}^{*} \oplus\left(\mathbb{R}^{n}\right)^{*} .
$$

Let $j: Z \hookrightarrow \mathbb{C}^{d}$ be the inclusion map, and let

$$
\operatorname{pr}_{1}:\left(\mathbb{R}^{d}\right)^{*} \longrightarrow \mathfrak{n}^{*} \quad \text { and } \quad \operatorname{pr}_{2}:\left(\mathbb{R}^{d}\right)^{*} \longrightarrow\left(\mathbb{R}^{n}\right)^{*}
$$

be the projection maps. The map

$$
\operatorname{pr}_{2} \circ \phi \circ j: Z \longrightarrow\left(\mathbb{R}^{n}\right)^{*}
$$

is constant on $N$-orbits. Thus there exists a map

$$
\mu: M_{\Delta} \longrightarrow\left(\mathbb{R}^{n}\right)^{*}
$$

such that

$$
\mu \circ p=\operatorname{pr}_{2} \circ \phi \circ j .
$$

The image of $\mu$ is equal to the image of $\operatorname{pr}_{2} \circ \phi \circ j$. We showed earlier that $\phi(Z)=\Delta^{\prime}$. Thus

$$
\text { Image of } \mu=\operatorname{pr}_{2}\left(\Delta^{\prime}\right)=\underbrace{\operatorname{pr}_{2} \circ \pi^{*}}_{\text {id }}(\Delta)=\Delta .
$$

Thus $\left(M_{\Delta}, \omega_{\Delta}\right)$ is the required toric manifold corresponding to $\Delta$.

\subsection{Idea Behind the Delzant Construction}

We use the idea that $\mathbb{R}^{d}$ is "universal" in the sense that any $n$-dimensional polytope $\Delta$ with $d$ facets can be obtained by intersecting the negative orthant $\mathbb{R}_{-}^{d}$ with an affine plane $A$. Given $\Delta$, to construct $A$ first write $\Delta$ as:

$$
\Delta=\left\{x \in \mathbb{R}^{n} \mid\left\langle x, v_{i}\right\rangle \leq \lambda_{i}, i=1, \ldots, d\right\} .
$$

Define

$$
\begin{aligned}
& \pi: \mathbb{R}^{d} \longrightarrow \mathbb{R}^{n} \quad \text { with dual map } \quad \pi^{*}: \mathbb{R}^{n} \longrightarrow \mathbb{R}^{d} . \\
& e_{i} \longmapsto v_{i}
\end{aligned}
$$

Then

$$
\pi^{*}-\lambda: \mathbb{R}^{n} \longrightarrow \mathbb{R}^{d}
$$


is an affine map, where $\lambda=\left(\lambda_{1}, \ldots, \lambda_{d}\right)$. Let $A$ be the image of $\pi^{*}-\lambda$. Then $A$ is an $n$-dimensional affine plane.

Claim. We have the equality $\left(\pi^{*}-\lambda\right)(\Delta)=\mathbb{R}_{-}^{d} \cap A$.

Proof. Let $x \in \mathbb{R}^{n}$. Then

$$
\begin{aligned}
\left(\pi^{*}-\lambda\right)(x) \in \mathbb{R}_{-}^{d} & \Longleftrightarrow\left\langle\pi^{*}(x)-\lambda, e_{i}\right\rangle \leq 0, \forall i \\
& \Longleftrightarrow\left\langle x, \pi\left(e_{i}\right)\right\rangle-\lambda_{i} \leq 0, \forall i \\
& \Longleftrightarrow\left\langle x, v_{i}\right\rangle \leq \lambda_{i}, \forall i \\
& \Longleftrightarrow x \in \Delta .
\end{aligned}
$$

We conclude that $\Delta \simeq \mathbb{R}_{-}^{d} \cap A$. Now $\mathbb{R}_{-}^{d}$ is the image of the moment map for the standard hamiltonian action of $\mathbb{T}^{d}$ on $\mathbb{C}^{\bar{d}}$

$$
\begin{aligned}
\phi: \mathbb{C}^{d} & \longrightarrow \mathbb{R}^{d} \\
\left(z_{1}, \ldots, z_{d}\right) & \longmapsto-\pi\left(\left|z_{1}\right|^{2}, \ldots,\left|z_{d}\right|^{2}\right) .
\end{aligned}
$$

\section{Facts.}

- The set $\phi^{-1}(A) \subset \mathbb{C}^{d}$ is a compact submanifold. Let $i: \phi^{-1}(A) \hookrightarrow \mathbb{C}^{d}$ denote inclusion. Then $i^{*} \omega_{0}$ is a closed 2-form which is degenerate. Its kernel is an integrable distribution. The corresponding foliation is called the null foliation.

- The null foliation of $i^{*} \omega_{0}$ is a principal fibration, so we take the quotient:

$$
\begin{gathered}
N \hookrightarrow \phi^{-1}(A) \\
\downarrow \\
M_{\Delta}=\phi^{-1}(A) / N
\end{gathered}
$$

Let $\omega_{\Delta}$ be the reduced symplectic form.

- The (non-effective) action of $\mathbb{T}^{d}=N \times \mathbb{T}^{n}$ on $\phi^{-1}(A)$ has a "moment map" with image $\phi\left(\phi^{-1}(A)\right)=\Delta$. (By "moment map" we mean a map satisfying the usual definition even though the closed 2 -form is not symplectic.)

Theorem 29.4 For any $x \in \Delta$, we have that $\mu^{-1}(x)$ is a single $\mathbb{T}^{n}$-orbit.

Proof. Exercise.

First consider the standard $\mathbb{T}^{d}$-action on $\mathbb{C}^{d}$ with moment map $\phi: \mathbb{C}^{d} \rightarrow \mathbb{R}^{d}$. Show that $\phi^{-1}(y)$ is a single $\mathbb{T}^{d}$-orbit for any $y \in \phi\left(\mathbb{C}^{d}\right)$. Now observe that

$$
y \in \Delta^{\prime}=\pi^{*}(\Delta) \Longleftrightarrow \phi^{-1}(y) \subseteq Z \text {. }
$$


Suppose that $y=\pi^{*}(x)$. Show that $\mu^{-1}(x)=\phi^{-1}(y) / N$. But $\phi^{-1}(y)$ is a single $\mathbb{T}^{d}$-orbit where $\mathbb{T}^{d}=N \times \mathbb{T}^{n}$, hence $\mu^{-1}(x)$ is a single $\mathbb{T}^{n}$-orbit.

Therefore, for toric manifolds, $\Delta$ is the orbit space.

Now $\Delta$ is a manifold with corners. At every point $p$ in a face $F$, the tangent space $T_{p} \Delta$ is the subspace of $\mathbb{R}^{n}$ tangent to $F$. We can visualize $\left(M_{\Delta}, \omega_{\Delta}, \mathbb{T}^{n}, \mu\right)$ from $\Delta$ as follows. First take the product $\mathbb{T}^{n} \times \Delta$. Let $p$ lie in the interior of $\mathbb{T}^{n} \times \Delta$. The tangent space at $p$ is $\mathbb{R}^{n} \times\left(\mathbb{R}^{n}\right)^{*}$. Define $\omega_{p}$ by:

$$
\omega_{p}(v, \xi)=\xi(v)=-\omega_{p}(\xi, v) \quad \text { and } \quad \omega_{p}\left(v, v^{\prime}\right)=\omega\left(\xi, \xi^{\prime}\right)=0 .
$$

for all $v, v^{\prime} \in \mathbb{R}^{n}$ and $\xi, \xi^{\prime} \in\left(\mathbb{R}^{n}\right)^{*}$. Then $\omega$ is a closed nondegenerate 2 -form on the interior of $\mathbb{T}^{n} \times \Delta$. At the corner there are directions missing in $\left(\mathbb{R}^{n}\right)^{*}$, so $\omega$ is a degenerate pairing. Hence, we need to eliminate the corresponding directions in $\mathbb{R}^{n}$. To do this, we collapse the orbits corresponding to subgroups of $\mathbb{T}^{n}$ generated by directions orthogonal to the annihilator of that face.

Example. Consider

$$
\left(S^{2}, \omega=d \theta \wedge d h, S^{1}, \mu=h\right),
$$

where $S^{1}$ acts on $S^{2}$ by rotation. The image of $\mu$ is the line segment $I=[-1,1]$. The product $S^{1} \times I$ is an open-ended cylinder. By collapsing each end of the cylinder to a point, we recover the 2 -sphere.

Exercise. Build $\mathbb{C P}^{2}$ from $\mathbb{T}^{2} \times \Delta$ where $\Delta$ is a right-angled isosceles triangle. $\diamond$

Finally, $\mathbb{T}^{n}$ acts on $\mathbb{T}^{n} \times \Delta$ by multiplication on the $\mathbb{T}^{n}$ factor. The moment map for this action is projection onto the $\Delta$ factor. 


\section{Homework 22: Delzant Theorem}

1. (a) Consider the standard $\left(S^{1}\right)^{3}$-action on $\mathbb{C P}^{3}$ :

$$
\left(e^{i \theta_{1}}, e^{i \theta_{2}}, e^{i \theta_{3}}\right) \cdot\left[z_{0}, z_{1}, z_{2}, z_{3}\right]=\left[z_{0}, e^{i \theta_{1}} z_{1}, e^{i \theta_{2}} z_{2}, e^{i \theta_{3}} z_{3}\right] .
$$

Exhibit explicitly the subsets of $\mathbb{C P}^{3}$ for which the stabilizer under this action is $\{1\}, S^{1},\left(S^{1}\right)^{2}$ and $\left(S^{1}\right)^{3}$. Show that the images of these subsets under the moment map are the interior, the facets, the edges and the vertices, respectively.

(b) Classify all 2-dimensional Delzant polytopes with 4 vertices, up to translation and the action of $\mathrm{SL}(2 ; \mathbb{Z})$.

Hint: By a linear transformation in $\mathrm{SL}(2 ; \mathbb{Z})$, you can make one of the angles in the polytope into a square angle. Check that automatically another angle also becomes $90^{\circ}$.

(c) What are all the 4-dimensional symplectic toric manifolds that have four fixed points?

2. Take a Delzant polytope in $\mathbb{R}^{n}$ with a vertex $p$ and with primitive (inwardpointing) edge vectors $u_{1}, \ldots, u_{n}$ at $p$. Chop off the corner to obtain a new polytope with the same vertices except $p$, and with $p$ replaced by $n$ new vertices:

$$
p+\varepsilon u_{j}, \quad j=1, \ldots, n,
$$

where $\varepsilon$ is a small positive real number. Show that this new polytope is also Delzant. The corresponding toric manifold is the $\varepsilon$-symplectic blowup of the original one.

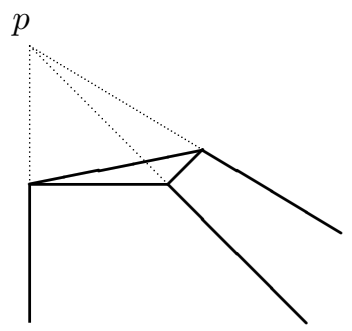


3. The toric 4-manifold $\mathcal{H}_{n}$ corresponding to the polygon with vertices $(0,0)$, $(n+1,0),(0,1)$ and $(1,1)$, for $n$ a nonnegative integer, is called a Hirzebruch surface.
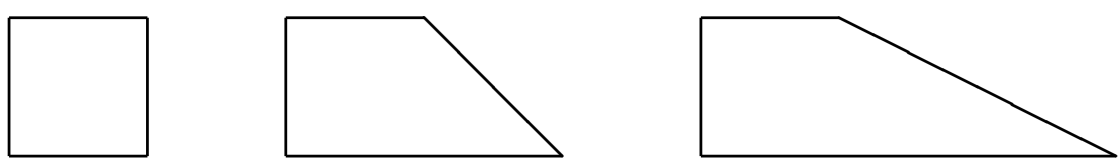

(a) What is the manifold $\mathcal{H}_{0}$ ? What is the manifold $\mathcal{H}_{1}$ ?

Hint:

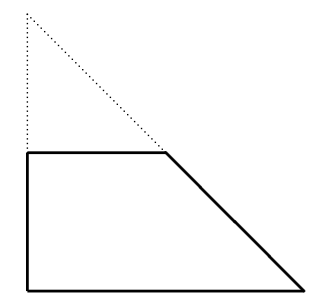

(b) Construct the manifold $\mathcal{H}_{n}$ by symplectic reduction of $\mathbb{C}^{4}$ with respect to an action of $\left(S^{1}\right)^{2}$.

(c) Exhibit $\mathcal{H}_{n}$ as a $\mathbb{C P}^{1}$-bundle over $\mathbb{C P}^{1}$.

4. Which $2 n$-dimensional toric manifolds have exactly $n+1$ fixed points? 


\section{Duistermaat-Heckman Theorems}

\subsection{Duistermaat-Heckman Polynomial}

Let $\left(M^{2 n}, \omega\right)$ be a symplectic manifold. Then $\frac{\omega^{n}}{n !}$ is the symplectic volume form.

Definition 30.1 The Liouville measure (or symplectic measure) of a Borel sub$\operatorname{set}^{17} \mathcal{U}$ of $M$ is

$$
m_{\omega}(\mathcal{U})=\int_{\mathcal{U}} \frac{\omega^{n}}{n !} .
$$

Let $G$ be a torus. Suppose that $(M, \omega, G, \mu)$ is a hamiltonian $G$-space, and that the moment map $\mu$ is proper.

Definition 30.2 The Duistermaat-Heckman measure, $m_{D H}$, on $\mathfrak{g}^{*}$ is the pushforward of $m_{\omega}$ by $\mu: M \rightarrow \mathfrak{g}^{*}$. That is,

$$
m_{D H}(U)=\left(\mu_{*} m_{\omega}\right)(U)=\int_{\mu^{-1}(U)} \frac{\omega^{n}}{n !}
$$

for any Borel subset $U$ of $\mathfrak{g}^{*}$.

For a compactly-supported function $h \in C^{\infty}\left(\mathfrak{g}^{*}\right)$, we define its integral with respect to the Duistermaat-Heckman measure to be

$$
\int_{\mathfrak{g}^{*}} h d m_{D H}=\int_{M}(h \circ \mu) \frac{\omega^{n}}{n !} .
$$

On $\mathfrak{g}^{*}$ regarded as a vector space, say $\mathbb{R}^{n}$, there is also the Lebesgue (or euclidean) measure, $m_{0}$. The relation between $m_{D H}$ and $m_{0}$ is governed by the Radon-Nikodym derivative, denoted by $\frac{d m_{D H}}{d m_{0}}$, which is a generalized function satisfying

$$
\int_{\mathfrak{g}^{*}} h d m_{D H}=\int_{\mathfrak{g}^{*}} h \frac{d m_{D H}}{d m_{0}} d m_{0} .
$$

Theorem 30.3 (Duistermaat-Heckman, 1982 [31]) The Duistermaat-Heckman measure is a piecewise polynomial multiple of Lebesgue (or euclidean) measure $m_{0}$ on $\mathfrak{g}^{*} \simeq \mathbb{R}^{n}$, that is, the Radon-Nikodym derivative

$$
f=\frac{d m_{D H}}{d m_{0}}
$$

is piecewise polynomial. More specifically, for any Borel subset $U$ of $\mathfrak{g}^{*}$,

$$
m_{D H}(U)=\int_{U} f(x) d x,
$$

where $d x=d m_{0}$ is the Lebesgue volume form on $U$ and $f: \mathfrak{g}^{*} \simeq \mathbb{R}^{n} \rightarrow \mathbb{R}$ is polynomial on any region consisting of regular values of $\mu$.

\footnotetext{
${ }^{17}$ The set $\mathcal{B}$ of Borel subsets is the $\sigma$-ring generated by the set of compact subsets, i.e., if $A, B \in \mathcal{B}$, then $A \backslash B \in \mathcal{B}$, and if $A_{i} \in \mathcal{B}, i=1,2, \ldots$, then $\cup_{i=1}^{\infty} A_{i} \in \mathcal{B}$.
} 
The proof of Theorem 30.3 for the case $G=S^{1}$ is in Section 30.3. The proof for the general case, which follows along similar lines, can be found in, for instance, [54], besides the original articles.

The Radon-Nikodym derivative $f$ is called the Duistermaat-Heckman polynomial. In the case of a toric manifold, the Duistermaat-Heckman polynomial is a universal constant equal to $(2 \pi)^{n}$ when $\Delta$ is $n$-dimensional. Thus the symplectic volume of $\left(M_{\Delta}, \omega_{\Delta}\right)$ is $(2 \pi)^{n}$ times the euclidean volume of $\Delta$.

Example. Consider $\left(S^{2}, \omega=d \theta \wedge d h, S^{1}, \mu=h\right)$. The image of $\mu$ is the interval $[-1,1]$. The Lebesgue measure of $[a, b] \subseteq[-1,1]$ is

$$
m_{0}([a, b])=b-a .
$$

The Duistermaat-Heckman measure of $[a, b]$ is

$$
m_{D H}([a, b])=\int_{\left\{(\theta, h) \in S^{2} \mid a \leq h \leq b\right\}} d \theta d h=2 \pi(b-a) .
$$

Consequently, the spherical area between two horizontal circles depends only on the vertical distance between them, a result which was known to Archimedes around $230 \mathrm{BC}$.

Corollary 30.4 For the standard hamiltonian action of $S^{1}$ on $\left(S^{2}, \omega\right)$, we have

$$
m_{D H}=2 \pi m_{0} \text {. }
$$

\subsection{Local Form for Reduced Spaces}

Let $(M, \omega, G, \mu)$ be a hamiltonian $G$-space, where $G$ is an $n$-torus. ${ }^{18}$ Assume that $\mu$ is proper. If $G$ acts freely on $\mu^{-1}(0)$, it also acts freely on nearby levels $\mu^{-1}(t)$, $t \in \mathfrak{g}^{*}$ and $t \approx 0$. Consider the reduced spaces

$$
M_{\text {red }}=\mu^{-1}(0) / G \quad \text { and } \quad M_{t}=\mu^{-1}(t) / G
$$

with reduced symplectic forms $\omega_{\text {red }}$ and $\omega_{t}$. What is the relation between these reduced spaces as symplectic manifolds?

For simplicity, we will assume $G$ to be the circle $S^{1}$. Let $Z=\mu^{-1}(0)$ and let $i: Z \hookrightarrow M$ be the inclusion map. We fix a connection form $\alpha \in \Omega^{1}(Z)$ for the principal bundle

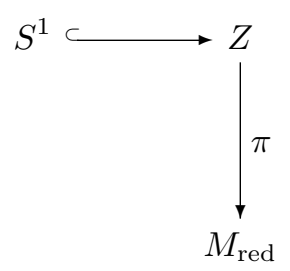

\footnotetext{
${ }^{18}$ The discussion in this section may be extended to hamiltonian actions of other compact Lie groups, not necessarily tori; see [54, Exercises 2.1-2.10].
} 
that is, $\mathcal{L}_{X \#} \alpha=0$ and $\imath_{X \#} \alpha=1$, where $X^{\#}$ is the infinitesimal generator for the $S^{1}$-action. From $\alpha$ we construct a 2 -form on the product manifold $Z \times(-\varepsilon, \varepsilon)$ by the recipe

$$
\sigma=\pi^{*} \omega_{\text {red }}-d(x \alpha),
$$

$x$ being a linear coordinate on the interval $(-\varepsilon, \varepsilon) \subset \mathbb{R} \simeq \mathfrak{g}^{*}$. (By abuse of notation, we shorten the symbols for forms on $Z \times(-\varepsilon, \varepsilon)$ which arise by pullback via projection onto each factor.)

Lemma 30.5 The 2-form $\sigma$ is symplectic for $\varepsilon$ small enough.

Proof. The form $\sigma$ is clearly closed. At points where $x=0$, we have

$$
\left.\sigma\right|_{x=0}=\pi^{*} \omega_{\text {red }}+\alpha \wedge d x
$$

which satisfies

$$
\left.\sigma\right|_{x=0}\left(X^{\#}, \frac{\partial}{\partial x}\right)=1
$$

so $\sigma$ is nondegenerate along $Z \times\{0\}$. Since nondegeneracy is an open condition, we conclude that $\sigma$ is nondegenerate for $x$ in a sufficiently small neighborhood of 0 .

Notice that $\sigma$ is invariant with respect to the $S^{1}$-action on the first factor of $Z \times(-\varepsilon, \varepsilon)$. In fact, this $S^{1}$-action is hamiltonian with moment map given by projection onto the second factor,

$$
x: Z \times(-\varepsilon, \varepsilon) \longrightarrow(-\varepsilon, \varepsilon),
$$

as is easily verified:

$$
\imath_{X} \#=-\imath_{X} \# d(x \alpha)=-\underbrace{\mathcal{L}_{X^{\#}}(x \alpha)}_{0}+d \underbrace{\imath_{X \#}(x \alpha)}_{x}=d x .
$$

Lemma 30.6 There exists an equivariant symplectomorphism between a neighborhood of $Z$ in $M$ and a neighborhood of $Z \times\{0\}$ in $Z \times(-\varepsilon, \varepsilon)$, intertwining the two moment maps, for $\varepsilon$ small enough.

Proof. The inclusion $i_{0}: Z \hookrightarrow Z \times(-\varepsilon, \varepsilon)$ as $Z \times\{0\}$ and the natural inclusion $i: Z \hookrightarrow M$ are $S^{1}$-equivariant coisotropic embeddings. Moreover, they satisfy $i_{0}^{*} \sigma=i^{*} \omega$ since both sides are equal to $\pi^{*} \omega_{\text {red }}$, and the moment maps coincide on $Z$ because $i_{0}^{*} x=0=i^{*} \mu$. Replacing $\varepsilon$ by a smaller positive number if necessary, the result follows from the equivariant version of the coisotropic embedding theorem stated in Section 8.3. ${ }^{19}$

\footnotetext{
${ }^{19}$ The equivariant version of Theorem 8.6 needed for this purpose may be phrased as follows: Let $\left(M_{0}, \omega_{0}\right),\left(M_{1}, \omega_{1}\right)$ be symplectic manifolds of dimension $2 n, G$ a compact Lie group acting on $\left(M_{i}, \omega_{i}\right), i=0,1$, in a hamiltonian way with moment maps $\mu_{0}$ and $\mu_{1}$, respectively, $Z$ a manifold of dimension $k \geq n$ with a $G$-action, and $\iota_{i}: Z \hookrightarrow M_{i}, i=0,1, G$-equivariant coisotropic embeddings. Suppose that $\iota_{0}^{*} \omega_{0}=\iota_{1}^{*} \omega_{1}$ and $\iota_{0}^{*} \mu_{0}=\iota_{1}^{*} \mu_{1}$. Then there exist $G$ invariant neighborhoods $\mathcal{U}_{0}$ and $\mathcal{U}_{1}$ of $\iota_{0}(Z)$ and $\iota_{1}(Z)$ in $M_{0}$ and $M_{1}$, respectively, and a $G$-equivariant symplectomorphism $\varphi: \mathcal{U}_{0} \rightarrow \mathcal{U}_{1}$ such that $\varphi \circ \iota_{0}=\iota_{1}$ and $\mu_{0}=\varphi^{*} \mu_{1}$.
} 
Therefore, in order to compare the reduced spaces

$$
M_{t}=\mu^{-1}(t) / S^{1}, \quad t \approx 0,
$$

we can work in $Z \times(-\varepsilon, \varepsilon)$ and compare instead the reduced spaces

$$
x^{-1}(t) / S^{1}, \quad t \approx 0 .
$$

Proposition 30.7 The reduced space $\left(M_{t}, \omega_{t}\right)$ is symplectomorphic to

$$
\left(M_{\text {red }}, \omega_{\text {red }}-t \beta\right) \text {, }
$$

where $\beta$ is the curvature form of the connection $\alpha$.

Proof. By Lemma 30.6, $\left(M_{t}, \omega_{t}\right)$ is symplectomorphic to the reduced space at level $t$ for the hamiltonian space $\left(Z \times(-\varepsilon, \varepsilon), \sigma, S^{1}, x\right)$. Since $x^{-1}(t)=Z \times\{t\}$, where $S^{1}$ acts on the first factor, all the manifolds $x^{-1}(t) / S^{1}$ are diffeomorphic to $Z / S^{1}=M_{\text {red }}$. As for the symplectic forms, let $\iota_{t}: Z \times\{t\} \hookrightarrow Z \times(-\varepsilon, \varepsilon)$ be the inclusion map. The restriction of $\sigma$ to $Z \times\{t\}$ is

$$
\iota_{t}^{*} \sigma=\pi^{*} \omega_{\text {red }}-t d \alpha .
$$

By definition of curvature, $d \alpha=\pi^{*} \beta$. Hence, the reduced symplectic form on $x^{-1}(t) / S^{1}$ is

$$
\omega_{\text {red }}-t \beta
$$

In loose terms, Proposition 30.7 says that the reduced forms $\omega_{t}$ vary linearly in $t$, for $t$ close enough to 0 . However, the identification of $M_{t}$ with $M_{\text {red }}$ as abstract manifolds is not natural. Nonetheless, any two such identifications are isotopic. By the homotopy invariance of de Rham classes, we obtain:

Theorem 30.8 (Duistermaat-Heckman, 1982 [31]) The cohomology class of the reduced symplectic form $\left[\omega_{t}\right]$ varies linearly in $t$. More specifically,

$$
\left[\omega_{t}\right]=\left[\omega_{\mathrm{red}}\right]+t c,
$$

where $c=[-\beta] \in H_{\mathrm{deRham}}^{2}\left(M_{\mathrm{red}}\right)$ is the first Chern class of the $S^{1}$-bundle $Z \rightarrow$ $M_{\text {red }}$.

Remark on conventions. Connections on principal bundles are Lie algebra-valued 1 -forms; cf. Section 25.2. Often the Lie algebra of $S^{1}$ is identified with $2 \pi i \mathbb{R}$ under the exponential map exp $: \mathfrak{g} \simeq 2 \pi i \mathbb{R} \rightarrow S^{1}, \xi \mapsto e^{\xi}$. Given a principal $S^{1}$-bundle, by this identification the infinitesimal action maps the generator $2 \pi i$ of $2 \pi i \mathbb{R}$ to the generating vector field $X^{\#}$. A connection form $A$ is then an imaginary-valued 1-form on the total space satisfying $\mathcal{L}_{X \#} A=0$ and $\imath_{X \#} A=2 \pi i$. Its curvature form $B$ is an imaginary-valued 2-form on the base satisfying $\pi^{*} B=d A$. By the Chern-Weil isomorphism, the first Chern class of the principal $S^{1}$-bundle is $c=\left[\frac{i}{2 \pi} B\right]$. 
In this lecture, we identify the Lie algebra of $S^{1}$ with $\mathbb{R}$ and implicitly use the exponential map $\exp : \mathfrak{g} \simeq \mathbb{R} \rightarrow S^{1}, t \mapsto e^{2 \pi i t}$. Hence, given a principal $S^{1}$ bundle, the infinitesimal action maps the generator 1 of $\mathbb{R}$ to $X^{\#}$, and here a connection form $\alpha$ is an ordinary 1 -form on the total space satisfying $\mathcal{L}_{X \#} \alpha=0$ and $\imath_{X \#} \alpha=1$. The curvature form $\beta$ is an ordinary 2 -form on the base satisfying $\pi^{*} \beta=d \alpha$. Consequently, we have $A=2 \pi i \alpha, B=2 \pi i \beta$ and the first Chern class is given by $c=[-\beta]$.

\subsection{Variation of the Symplectic Volume}

Let $\left(M, \omega, S^{1}, \mu\right)$ be a hamiltonian $S^{1}$-space of dimension $2 n$ and let $\left(M_{x}, \omega_{x}\right)$ be its reduced space at level $x$. Proposition 30.7 or Theorem 30.8 imply that, for $x$ in a sufficiently narrow neighborhood of 0 , the symplectic volume of $M_{x}$,

$$
\operatorname{vol}\left(M_{x}\right)=\int_{M_{x}} \frac{\omega_{x}^{n-1}}{(n-1) !}=\int_{M_{\mathrm{red}}} \frac{\left(\omega_{\mathrm{red}}-x \beta\right)^{n-1}}{(n-1) !},
$$

is a polynomial in $x$ of degree $n-1$. This volume can be also expressed as

$$
\operatorname{vol}\left(M_{x}\right)=\int_{Z} \frac{\pi^{*}\left(\omega_{\text {red }}-x \beta\right)^{n-1}}{(n-1) !} \wedge \alpha .
$$

Recall that $\alpha$ is a chosen connection form for the $S^{1}$-bundle $Z \rightarrow M_{\text {red }}$ and $\beta$ is its curvature form.

Now we go back to the computation of the Duistermaat-Heckman measure. For a Borel subset $U$ of $(-\varepsilon, \varepsilon)$, the Duistermaat-Heckman measure is, by definition,

$$
m_{D H}(U)=\int_{\mu^{-1}(U)} \frac{\omega^{n}}{n !} .
$$

Using the fact that $\left(\mu^{-1}(-\varepsilon, \varepsilon), \omega\right)$ is symplectomorphic to $(Z \times(-\varepsilon, \varepsilon), \sigma)$ and, moreover, they are isomorphic as hamiltonian $S^{1}$-spaces, we obtain

$$
m_{D H}(U)=\int_{Z \times U} \frac{\sigma^{n}}{n !}
$$

Since $\sigma=\pi^{*} \omega_{\text {red }}-d(x \alpha)$, its power is

$$
\sigma^{n}=n\left(\pi^{*} \omega_{\text {red }}-x d \alpha\right)^{n-1} \wedge \alpha \wedge d x .
$$

By the Fubini theorem, we then have

$$
m_{D H}(U)=\int_{U}\left[\int_{Z} \frac{\pi^{*}\left(\omega_{\mathrm{red}}-x \beta\right)^{n-1}}{(n-1) !} \wedge \alpha\right] \wedge d x .
$$

Therefore, the Radon-Nikodym derivative of $m_{D H}$ with respect to the Lebesgue measure, $d x$, is

$$
f(x)=\int_{Z} \frac{\pi^{*}\left(\omega_{\text {red }}-x \beta\right)^{n-1}}{(n-1) !} \wedge \alpha=\operatorname{vol}\left(M_{x}\right) .
$$


The previous discussion proves that, for $x \approx 0, f(x)$ is a polynomial in $x$. The same holds for a neighborhood of any other regular value of $\mu$, because we may change the moment map $\mu$ by an arbitrary additive constant. 


\section{Homework 23: $S^{1}$-Equivariant Cohomology}

1. Let $M$ be a manifold with a circle action and $X^{\#}$ the vector field on $M$ generated by $S^{1}$. The algebra of $S^{1}$-equivariant forms on $M$ is the algebra of $S^{1}$-invariant forms on $M$ tensored with complex polynomials in $x$,

$$
\Omega_{S^{1}}^{\bullet}(M):=\left(\Omega^{\bullet}(M)\right)^{S^{1}} \otimes_{\mathbb{R}} \mathbb{C}[x] .
$$

The product $\wedge$ on $\Omega_{S^{1}}^{\bullet}(M)$ combines the wedge product on $\Omega^{\bullet}(M)$ with the product of polynomials on $\mathbb{C}[x]$.

(a) We grade $\Omega_{S^{1}}^{\bullet}(M)$ by adding the usual grading on $\Omega^{\bullet}(M)$ to a grading on $\mathbb{C}[x]$ where the monomial $x$ has degree 2 . Check that $\left(\Omega_{S^{1}}^{\bullet}(M), \wedge\right)$ is then a supercommutative graded algebra, i.e.,

$$
\underline{\alpha} \wedge \underline{\beta}=(-1)^{\operatorname{deg} \underline{\alpha} \cdot \operatorname{deg} \underline{\beta} \underline{\beta}} \wedge \underline{\alpha}
$$

for elements of pure degree $\underline{\alpha}, \underline{\beta} \in \Omega_{S^{1}}^{\bullet}(M)$.

(b) On $\Omega_{S^{1}}^{\bullet}(M)$ we define an operator

$$
d_{S^{1}}:=d \otimes 1-\imath_{X^{\#}} \otimes x .
$$

In other words, for an elementary form $\underline{\alpha}=\alpha \otimes p(x)$,

$$
d_{S^{1}} \underline{\alpha}=d \alpha \otimes p(x)-\imath_{X \#} \alpha \otimes x p(x) .
$$

The operator $d_{S^{1}}$ is called the Cartan differentiation. Show that $d_{S^{1}}$ is a superderivation of degree 1 , i.e., check that it increases degree by 1 and that it satisfies the super Leibniz rule:

$$
d_{S^{1}}(\underline{\alpha} \wedge \underline{\beta})=\left(d_{S^{1}} \underline{\alpha}\right) \wedge \underline{\beta}+(-1)^{\operatorname{deg} \underline{\alpha}} \underline{\alpha} \wedge d_{S^{1}} \underline{\beta} .
$$

(c) Show that $d_{S^{1}}^{2}=0$.

Hint: Cartan magic formula.

2. The previous exercise shows that the sequence

$$
0 \longrightarrow \Omega_{S^{1}}^{0}(M) \stackrel{d_{S^{1}}}{\longrightarrow} \Omega_{S^{1}}^{1}(M) \stackrel{d_{S^{1}}}{\longrightarrow} \Omega_{S^{1}}^{2}(M) \stackrel{d_{S^{1}}}{\longrightarrow} \ldots
$$

forms a graded complex whose cohomology is called the equivariant cohomology ${ }^{20}$ of $M$ for the given action of $S^{1}$. The $k$ th equivariant cohomology group of $M$ is

$$
H_{\mathrm{S}^{1}}^{k}(M):=\frac{\operatorname{ker} d_{S^{1}}: \Omega_{S^{1}}^{k} \longrightarrow \Omega_{S^{1}}^{k+1}}{\operatorname{im} d_{S^{1}}: \Omega_{S^{1}}^{k-1} \longrightarrow \Omega_{S^{1}}^{k}} .
$$

\footnotetext{
${ }^{20}$ The equivariant cohomology of a topological space $M$ endowed with a continuous action of a topological group $G$ is, by definition, the cohomology of the diagonal quotient $(M \times E G) / G$, where $E G$ is the universal bundle of $G$, i.e., $E G$ is a contractible space where $G$ acts freely. H. Cartan $[21,59]$ showed that, for the action of a compact Lie group $G$ on a manifold $M$, the de Rham model $\left(\Omega_{G}^{\bullet}(M), d_{G}\right)$ computes the equivariant cohomology, where $\Omega_{G}^{\bullet}(M)$ are the $G$-equivariant forms on $M .[8,9,29,54]$ explain equivariant cohomology in the symplectic context and [59] discusses equivariant de Rham theory and many applications.
} 
(a) What is the equivariant cohomology of a point?

(b) What is the equivariant cohomology of $S^{1}$ with its multiplication action on itself?

(c) Show that the equivariant cohomology of a manifold $M$ with a free $S^{1}$ action is isomorphic to the ordinary cohomology of the quotient space $M / S^{1}$.

Hint: Let $\pi: M \rightarrow M / S^{1}$ be projection. Show that

$$
\begin{aligned}
\pi^{*}: H^{\bullet}\left(M / S^{1}\right) & \longrightarrow H_{S^{1}}(M) \\
{[\alpha] } & \longmapsto\left[\pi^{*} \alpha \otimes 1\right]
\end{aligned}
$$

is a well-defined isomorphism. It helps to choose a connection on the principal $S^{1}$-bundle $M \rightarrow M / S^{1}$, that is, a 1 -form $\theta$ on $M$ such that $\mathcal{L}_{X \#} \theta=0$ and $\imath_{X} \#=1$. Keep in mind that a form $\beta$ on $M$ is of type $\pi^{*} \alpha$ for some $\alpha$ if and only if it is basic, that is $\mathcal{L}_{X \#} \beta=0$ and $\imath_{X} \beta=0$.

3. Suppose that $(M, \omega)$ is a symplectic manifold with an $S^{1}$-action. Let $\mu \in$ $C^{\infty}(M)$ be a real function. Consider the equivariant form

$$
\underline{\omega}:=\omega \otimes 1+\mu \otimes x .
$$

Show that $\underline{\omega}$ is equivariantly closed, i.e., $d_{S^{1}} \underline{\omega}=0$ if and only if $\mu$ is a moment map. The equivariant form $\underline{\omega}$ is called the equivariant symplectic form.

4. Let $M^{2 n}$ be a compact oriented manifold, not necessarily symplectic, acted upon by $S^{1}$. Suppose that the set $M^{S^{1}}$ of fixed points for this action is finite. Let $\alpha^{(2 n)}$ be an $S^{1}$-invariant form which is the top degree part of an equivariantly closed form of even degree, that is, $\alpha^{(2 n)} \in \Omega^{2 n}(M)^{S^{1}}$ is such that there exists $\underline{\alpha} \in \Omega_{S^{1}}^{\bullet}(M)$ with

$$
\underline{\alpha}=\alpha^{(2 n)}+\alpha^{(2 n-2)}+\ldots+\alpha^{(0)}
$$

where $\alpha^{(2 k)} \in\left(\Omega^{2 k}(M)\right)^{S^{1}} \otimes \mathbb{C}[x]$ and $d_{S^{1}} \underline{\alpha}=0$.

(a) Show that the restriction of $\alpha^{(2 n)}$ to $M \backslash M^{S^{1}}$ is exact.

Hint: The generator $X^{\#}$ of the $S^{1}$-action does not vanish on $M \backslash M^{S^{1}}$. Hence, we can define a connection on $M \backslash M^{S^{1}}$ by $\theta(Y)=\frac{\left\langle Y, X^{\#}\right\rangle}{\left\langle X^{\#}, X^{\#}\right\rangle}$, where $\langle\cdot, \cdot\rangle$ is some $S^{1}$-invariant metric on $M$. Use $\theta \in \Omega^{1}\left(M \backslash M^{S^{1}}\right)$ to chase the primitive of $\alpha^{(2 n)}$ all the way up from $\alpha^{(0)}$.

(b) Compute the integral of $\alpha^{(2 n)}$ over $M$.

Hint: Stokes' theorem allows to localize the answer near the fixed points.

This exercise is a very special case of the Atiyah-Bott-Berline-Vergne localization theorem for equivariant cohomology [8, 14].

5. What is the integral of the symplectic form $\omega$ on a surface with a hamiltonian $S^{1}$-action, knowing that the $S^{1}$-action is free outside a finite set of fixed points?

Hint: Exercises 3 and 4. 


\section{References}

[1] Abraham, R., Marsden, J. E., Foundations of Mechanics, second edition, Addison-Wesley, Reading, 1978.

[2] Aebischer, B., Borer, M., Kälin, M., Leuenberger, Ch., Reimann, H.M., Symplectic Geometry. An Introduction Based on the Seminar in Bern, 1992, Progress in Mathematics 124, Birkhäuser Verlag, Basel, 1994.

[3] Arnold, V., Mathematical Methods of Classical Mechanics, Graduate Texts in Math. 60, Springer-Verlag, New York, 1978.

[4] Arnold, V., First steps of symplectic topology, VIIIth International Congress on Mathematical Physics (Marseille, 1986), 1-16, World Sci. Publishing, Singapore, 1987.

[5] Arnold, V., Givental, A., Symplectic geometry, Dynamical Systems IV, Symplectic Geometry and its Applications, edited by Arnold, V. and Novikov, S., Encyclopaedia of Mathematical Sciences 4, Springer-Verlag, Berlin-New York, 1990.

[6] Atiyah, M., Convexity and commuting Hamiltonians, Bull. London Math. Soc. 14 (1982), 1-15.

[7] Atiyah, M., Bott, R., The Yang-Mills equations over Riemann surfaces, Topology 23 (1984), 1-28. Philos. Trans. Roy. Soc. London 308 (1983), 523-615.

[8] Atiyah, M., Bott, R., The moment map and equivariant cohomology, Topology 23 (1984), 1-28.

[9] Audin, M., The Topology of Torus Actions on Symplectic Manifolds, Progress in Mathematics 93, Birkhäuser Verlag, Basel, 1991.

[10] Audin, M., Spinning Tops. A Course on Integrable Systems, Cambridge Studies in Advanced Mathematics 51, Cambridge University Press, Cambridge, 1996.

[11] Audin, M., Lafontaine, J., Eds., Holomorphic Curves in Symplectic Geometry, Progress in Mathematics 117, Birkhäuser Verlag, Basel, 1994.

[12] Auroux, D., Asymptotically holomorphic families of symplectic submanifolds, Geom. Funct. Anal. 7 (1997), 971-995.

[13] Berline, N., Getzler, E., Vergne, M., Heat Kernels and Dirac Operators, Grundlehren der Mathematischen Wissenschaften 298, Springer-Verlag, Berlin, 1992.

[14] Berline, N., Vergne, M., Classes caractéristiques équivariantes, formule de localisation en cohomologie équivariante, C. R. Acad. Sci. Paris Sér. I Math. 295 (1982), 539-541. 
[15] Berline, N., Vergne, M., Zéros d'un champ de vecteurs et classes caractéristiques équivariantes, Duke Math. J. 50 (1983), 539-549.

[16] Biran, P., A stability property of symplectic packing, Invent. Math. 136 (1999), 123-155.

[17] Bredon, G., Introduction to Compact Transformation Groups, Pure and Applied Mathematics 46, Academic Press, New York-London, 1972.

[18] Bott, R., Tu, L., Differential Forms in Algebraic Topology, Graduate Texts in Mathematics 82, Springer-Verlag, New York-Berlin, 1982.

[19] Cannas da Silva, A., Guillemin, V., Woodward, C., On the unfolding of folded symplectic structures, Math. Res. Lett. 7 (2000), 35-53.

[20] Cannas da Silva, A., Weinstein, A., Geometric Models for Noncommutative Algebras, Berkeley Mathematics Lecture Notes series, Amer. Math. Soc., Providence, 1999.

[21] Cartan, H., La transgression dans un groupe de Lie et dans un espace fibré principal, Colloque de Topologie (Espaces Fibrés), Bruxelles, 1950, 57-71, Masson et Cie., Paris, 1951.

[22] Chern, S.S., Complex Manifolds Without Potential Theory, with an appendix on the geometry of characteristic classes, second edition, Universitext, Springer-Verlag, New York-Heidelberg, 1979.

[23] Delzant, T., Hamiltoniens périodiques et images convexes de I'application moment, Bull. Soc. Math. France 116 (1988), 315-339.

[24] Donaldson, S., Symplectic submanifolds and almost-complex geometry, J. Differential Geom. 44 (1996), 666-705.

[25] Donaldson, S., Lefschetz fibrations in symplectic geometry, Proceedings of the International Congress of Mathematicians, vol. II (Berlin, 1998), Doc. Math. 1998, extra vol. II, 309-314.

[26] Donaldson, S., Lefschetz pencils on symplectic manifolds, J. Differential Geom. 53 (1999), 205-236.

[27] Donaldson, S., Kronheimer, P., The Geometry of Four-Manifolds, Oxford Mathematical Monographs, The Clarendon Press, Oxford University Press, New York, 1990.

[28] Duistermaat, J.J., On global action-angle coordinates, Comm. Pure Appl. Math. 33 (1980), 687-706.

[29] Duistermaat, J.J., Equivariant cohomology and stationary phase, Symplectic Geometry and Quantization (Sanda and Yokohama, 1993), edited by Maeda, Y., Omori, H. and Weinstein, A., 45-62, Contemp. Math. 179, Amer. Math. Soc., Providence, 1994. 
[30] Duistermaat, J.J., The Heat Kernel Lefschetz Fixed Point Formula for the Spin-c Dirac Operator, Progress in Nonlinear Differential Equations and their Applications 18, Birkhäuser Boston, Inc., Boston, 1996.

[31] Duistermaat, J.J., Heckman, G., On the variation in the cohomology of the symplectic form of the reduced phase space, Invent. Math. 69 (1982), 259268; Addendum, Invent. Math. 72 (1983), 153-158.

[32] Eliashberg, Y., Classification of overtwisted contact structures on 3-manifolds, Invent. Math. 98 (1989), 623-637.

[33] Eliashberg, Y., Contact 3-manifolds twenty years since J. Martinet's work, Ann. Inst. Fourier (Grenoble) 42 (1992), 165-192.

[34] Eliashberg, Y., Gromov, M., Lagrangian intersection theory: finitedimensional approach, Geometry of Differential Equations, 27-118, Amer. Math. Soc. Transl. Ser. 2, 186, Amer. Math. Soc., Providence, 1998.

[35] Eliashberg, Y., Thurston, W., Confoliations, University Lecture Series 13, Amer. Math. Soc., Providence, 1998.

[36] Eliashberg, Y., Traynor, L., Eds., Symplectic Geometry and Topology, lectures from the Graduate Summer School held in Park City, June 29-July 19, 1997, IAS/Park City Mathematics Series 7, Amer. Math. Soc., Providence, 1999.

[37] Fernández, M., Gotay, M., Gray, A., Compact parallelizable four-dimensional symplectic and complex manifolds, Proc. Amer. Math. Soc. 103 (1988), 12091212.

[38] Fulton, W., Introduction to Toric Varieties, Annals of Mathematics Studies 131, Princeton University Press, Princeton, 1993.

[39] Geiges, H., Applications of contact surgery, Topology 36 (1997), 1193-1220.

[40] Geiges, H., Gonzalo, J., Contact geometry and complex surfaces, Invent. Math. 121 (1995), 147-209.

[41] Ginzburg, V., Guillemin, V., Karshon, Y., Cobordism theory and localization formulas for Hamiltonian group actions, Internat. Math. Res. Notices 1996, 221-234.

[42] Ginzburg, V., Guillemin, V., Karshon, Y., The relation between compact and non-compact equivariant cobordisms, Tel Aviv Topology Conference: Rothenberg Festschrift (1998), 99-112, Contemp. Math., 231, Amer. Math. Soc., Providence, 1999.

[43] Giroux, E., Topologie de contact en dimension 3 (autour des travaux de Yakov Eliashberg), Séminaire Bourbaki 1992/93, Astérisque 216 (1993), 7-33.

[44] Giroux, E., Une structure de contact, même tendue, est plus ou moins tordue, Ann. Sci. École Norm. Sup. (4) 27 (1994), 697-705. 
[45] Givental, A., Periodic mappings in symplectic topology (Russian), Funktsional. Anal. i Prilozhen 23 (1989), 37-52, translation in Funct. Anal. Appl. 23 (1989), 287-300.

[46] Gompf, R., A new construction of symplectic manifolds, Ann. of Math. 142 (1995), 527-595.

[47] Gotay, M., On coisotropic imbeddings of presymplectic manifolds, Proc. Amer. Math. Soc. 84 (1982), 111-114.

[48] Griffiths, P., Harris, J., Principles of Algebraic Geometry, Chapter 0, reprint of the 1978 original, Wiley Classics Library, John Wiley \& Sons, Inc., New York, 1994.

[49] Gromov, M., Pseudoholomorphic curves in symplectic manifolds, Invent. Math. 82 (1985), 307-347.

[50] Gromov, M., Partial Differential Relations, Springer-Verlag, Berlin-New York, 1986.

[51] Gromov, M., Soft and hard symplectic geometry, Proceedings of the International Congress of Mathematicians 1 (Berkeley, Calif., 1986), 81-98, Amer. Math. Soc., Providence, 1987.

[52] Guggenheimer, H., Sur les variétés qui possèdent une forme extérieure quadratique fermée, C. R. Acad. Sci. Paris 232 (1951), 470-472.

[53] Guillemin, V., Course 18.966 - Geometry of Manifolds, M.I.T., Spring of 1992.

[54] Guillemin, V., Moment Maps and Combinatorial Invariants of Hamiltonian $T^{n}$-spaces, Progress in Mathematics 122, Birkhäuser, Boston, 1994.

[55] Guillemin, V., Pollack, A., Differential Topology, Prentice-Hall, Inc., Englewood Cliffs, N.J., 1974.

[56] Guillemin, V., Sternberg, S., Geometric Asymptotics, Math. Surveys and Monographs 14, Amer. Math. Soc., Providence, 1977.

[57] Guillemin, V., Sternberg, S., Convexity properties of the moment mapping, Invent. Math. 67 (1982), 491-513.

[58] Guillemin, V., Sternberg, S., Symplectic Techniques in Physics, second edition, Cambridge University Press, Cambridge, 1990.

[59] Guillemin, V., Sternberg, S., Supersymmetry and Equivariant de Rham Theory, with an appendix containing two reprints by $\mathrm{H}$. Cartan, Mathematics Past and Present, Springer-Verlag, Berlin, 1999.

[60] Hausmann, J.-C., Knutson, A., The cohomology ring of polygon spaces, Ann. Inst. Fourier (Grenoble) 48 (1998), 281-321. 
[61] Hausmann, J.-C., Knutson, A., Cohomology rings of symplectic cuts, Differential Geom. Appl. 11 (1999), 197-203.

[62] Hitchin, N., Segal, G., Ward, R., Integrable Systems. Twistors, Loop groups, and Riemann Surfaces Oxford Graduate Texts in Mathematics 4, The Clarendon Press, Oxford University Press, New York, 1999.

[63] Hofer, H., Pseudoholomorphic curves in symplectizations with applications to the Weinstein conjecture in dimension three, Invent. Math. 114 (1993), 515-563.

[64] Hofer, H. Viterbo, C., The Weinstein conjecture in the presence of holomorphic spheres, Comm. Pure Appl. Math. 45 (1992), 583-622.

[65] Hofer, H., Zehnder, E., Symplectic Invariants and Hamiltonian Dynamics, Birkhäuser Advanced Texts: Basler Lehrbücher, Birkhäuser Verlag, Basel, 1994.

[66] Hörmander, L., An Introduction to Complex Analysis in Several Variables, third edition, North-Holland Mathematical Library 7, North-Holland Publishing Co., Amsterdam-New York, 1990.

[67] Jacobson, N., Lie Algebras, republication of the 1962 original, Dover Publications, Inc., New York, 1979.

[68] Jeffrey, L., Kirwan, F., Localization for nonabelian group actions, Topology 34 (1995), 291-327.

[69] Kirwan, F., Cohomology of Quotients in Symplectic and Algebraic Geometry, Mathematical Notes 31, Princeton University Press, Princeton, 1984.

[70] Kodaira, K., On the structure of compact complex analytic surfaces, I, Amer. J. Math. 86 (1964), 751-798.

[71] Kronheimer, P., Developments in symplectic topology, Current Developments in Mathematics (Cambridge, 1998), 83-104, Int. Press, Somerville, 1999.

[72] Kronheimer, P., Mrowka, T., Monopoles and contact structures, Invent. Math. 130 (1997), 209-255.

[73] Lalonde, F., McDuff, D., The geometry of symplectic energy, Ann. of Math. (2) 141 (1995), 349-371.

[74] Lalonde, F., Polterovich, L., Symplectic diffeomorphisms as isometries of Hofer's norm, Topology 36 (1997), 711-727.

[75] Lerman, E., Meinrenken, E., Tolman, S., Woodward, C., Nonabelian convexity by symplectic cuts, Topology 37 (1998), 245-259.

[76] Marsden, J., Ratiu, T., Introduction to Mechanics and Symmetry. A Basic Exposition of Classical Mechanical Systems, Texts in Applied Mathematics 17, Springer-Verlag, New York, 1994. 
[77] Marsden, J., Weinstein, A., Reduction of symplectic manifolds with symmetry, Rep. Mathematical Phys. 5 (1974), 121-130.

[78] Martin, S., Symplectic quotients by a nonabelian group and by its maximal torus, arXiv:math/0001002.

[79] Martin, S., Transversality theory, cobordisms, and invariants of symplectic quotients, arXiv:math/0001001.

[80] Martinet, J., Formes de contact sur les variétés de dimension 3, Proceedings of Liverpool Singularities Symposium II (1969/1970), 142-163, Lecture Notes in Math. 209, Springer, Berlin, 1971.

[81] McDuff, D., Examples of simply-connected symplectic non-Kählerian manifolds, J. Differential Geom. 20 (1984), 267-277.

[82] McDuff, D., The local behaviour of holomorphic curves in almost complex 4-manifolds, J. Differential Geom. 34 (1991), 143-164.

[83] McDuff, D., Salamon, D., Introduction to Symplectic Topology, Oxford Mathematical Monographs, Oxford University Press, New York, 1995.

[84] Meinrenken, E., Woodward, C., Hamiltonian loop group actions and Verlinde factorization, J. Differential Geom. 50 (1998), 417-469.

[85] Meyer, K., Symmetries and integrals in mechanics, Dynamical Systems (Proc. Sympos., Univ. Bahia, Salvador, 1971), 259-272. Academic Press, New York, 1973.

[86] Milnor, J., Morse Theory, based on lecture notes by M. Spivak and R. Wells, Annals of Mathematics Studies 51, Princeton University Press, Princeton, 1963.

[87] Moser, J., On the volume elements on a manifold, Trans. Amer. Math. Soc. 120 (1965), 286-294.

[88] Mumford, D., Fogarty, J., Kirwan, F., Geometric Invariant Theory, Ergebnisse der Mathematik und ihrer Grenzgebiete 34, Springer-Verlag, Berlin, 1994.

[89] Newlander, A., Nirenberg, L., Complex analytic coordinates in almost complex manifolds, Ann. of Math. 65 (1957), 391-404.

[90] Salamon, D., Morse theory, the Conley index and Floer homology, Bull. London Math. Soc. 22 (1990), 113-140.

[91] Satake, I., On a generalization of the notion of manifold, Proc. Nat. Acad. Sci. U.S.A. 42 (1956), 359-363.

[92] Scott, P., The geometries of 3-manifolds, Bull. London Math. Soc. 15 (1983), 401-487. 
[93] Seidel, P., Lagrangian two-spheres can be symplectically knotted, J. Differential Geom. 52 (1999), 145-171.

[94] Sjamaar, R., Lerman, E., Stratified symplectic spaces and reduction, Ann. of Math. 134 (1991), 375-422.

[95] Souriau, J.-M. , Structure des Systèmes Dynamiques, Maîtrises de Mathématiques, Dunod, Paris 1970.

[96] Spivak, M., A Comprehensive Introduction to Differential Geometry, Vol. I, second edition, Publish or Perish, Inc., Wilmington, 1979.

[97] Taubes, C., The Seiberg-Witten invariants and symplectic forms, Math. Res. Lett. 1 (1994), 809-822.

[98] Taubes, C., More constraints on symplectic forms from Seiberg-Witten invariants, Math. Res. Lett. 2 (1995), 9-13.

[99] Taubes, C., The Seiberg-Witten and Gromov invariants, Math. Res. Lett. 2 (1995), 221-238.

[100] Thomas, C., Eliashberg, Y., Giroux, E., 3-dimensional contact geometry, Contact and Symplectic Geometry (Cambridge, 1994), 48-65, Publ. Newton Inst. 8. Cambridge University Press, Cambridge, 1996.

[101] Thurston, W., Some simple examples of symplectic manifolds, Proc. Amer. Math. Soc. 55 (1976), 467-468.

[102] Tolman, S., Weitsman, J., On semifree symplectic circle actions with isolated fixed points, Topology 39 (2000), 299-309.

[103] Viterbo, C., A proof of Weinstein's conjecture in $\mathbb{R}^{2 n}$, Ann. Inst. H. Poincaré Anal. Non Linéaire 4 (1987), 337-356.

[104] Weinstein, A., Symplectic manifolds and their Lagrangian submanifolds, Advances in Math. 6 (1971), 329-346.

[105] Weinstein, A., Lectures on Symplectic Manifolds, Regional Conference Series in Mathematics 29, Amer. Math. Soc., Providence, 1977.

[106] Weinstein, A., On the hypotheses of Rabinowitz' periodic orbit theorems, J. Differential Equations 33 (1979), 353-358.

[107] Weinstein, A., Neighborhood classification of isotropic embeddings, J. Differential Geom. 16 (1981), 125-128.

[108] Weinstein, A., Symplectic geometry, Bull. Amer. Math. Soc. (N.S.) 5 (1981), $1-13$.

[109] Wells, R.O., Differential Analysis on Complex Manifolds, second edition, Graduate Texts in Mathematics 65, Springer-Verlag, New York-Berlin, 1980. 
[110] Weyl, H., The Classical Groups. Their Invariants and Representations, Princeton Landmarks in Mathematics, Princeton University Press, Princeton, 1997.

[111] Witten, E., Two-dimensional gauge theories revisited, J. Geom. Phys. 9 (1992), 303-368.

[112] Woodward, C., Multiplicity-free Hamiltonian actions need not be Kähler, Invent. Math. 131 (1998), 311-319.

[113] Woodward, C., Spherical varieties and existence of invariant Kähler structures, Duke Math. J. 93 (1998), 345-377. 


\section{Index}

action

adjoint, 131, 137

coadjoint, 131, 137

coordinates, 111

definition, 128

effective, 170

free, 135

gauge group, 158

hamiltonian, 129, 130, 133, 164

infinitesimal, 156, 164

locally free, 135

minimizing, 115, 120

of a path, 114, 115, 119

principle of least action, 114

smooth, 128

symplectic, 129

transitive, 135

action-angle coordinates, 111

adapted coordinates, 18

adjoint

action, 131, 137

representation, 130, 131

almost complex manifold, 70

almost complex structure

compatibility, 70

contractibility, 77

definition, 70

integrability, 75, 82

three geometries, 67

almost complex submanifold, 76

almost symplectic manifold, 74

angle coordinates, 110

angular momentum, 137, 138

$(J$-)anti-holomorphic tangent vectors, 78 antisymmetry, 108

arc-length, 25

Archimedes, 192

Arnold

Arnold-Liouville theorem, 110

Atiyah

conjecture, 33, 55, 56

Atiyah-Guillemin-Sternberg theorem, 169 moduli space, 158

Yang-Mills theory, 155

Banyaga theorem, 92

base, 155

basis

for skew-symmetric bilinear maps, 3

Beltrami

Laplace-Beltrami operator, 98

Betti number, 100

biholomorphic map, 83

bilinear map, see skew-symmetric bilinear map

billiards, 30

Birkhoff

Poincaré-Birkhoff theorem, 33

blowup, 189

Borel subset, 191

Bott

moduli space, 158

Morse-Bott function, 174

Yang-Mills theory, 155

bracket

Lie, 108

Poisson, 108, 110, 134, 164

$C^{1}$-topology, 53, 54

canonical

symplectic form on a coadjoint or-

bit, 139, 150, 162

symplectomorphism, 12

canonical form on $T^{*} X$

coordinate definition, 9,10

intrinsic definition, 10

naturality, 11

Cartan

differentiation, 197

magic formula, 36, 40, 44

Cauchy-Riemann equations, 84

characteristic distribution, 53

chart

complex, 83

Darboux, 7 
Chern

first Chern class, 194

Chevalley cohomology, 165

Christoffel

equations, 120

symbols, 120

circle bundle, 159

classical mechanics, 107

coadjoint

action, 131, 137, 162

orbit, 139, 162

representation, 130, 131

codifferential, 98

cohomology

$S^{1}$-equivariant, 197

Chevalley, 165

de Rham, 13, 39

Dolbeault, 81

equivariant, 197

Lie algebra, 165

coindex, 174, 175

coisotropic

embedding, 49, 53

subspace, 8

commutator ideal, 165

comoment map, 133, 134, 164

compatible

almost complex structure, 70, 74

complex structure, 68

linear structures, 72

triple, 70,75

complete vector field, 129

completely integrable system, 110

complex

atlas, 89

chart, 83

differentials, 86,87

Hodge theory, 99

manifold, 83

projective space, $89,95,96,103$, $136,168,181$

complex structure

compatibility, 68,77

on a vector space, 68

polar decomposition, 69

complex surface, 103 complex torus, 103

complex vector space, 68

complex-antilinear cotangent vectors, 79

complex-linear cotangent vectors, 79

complex-valued form, 79

conehead orbifold, 150

configuration space, 107, 113

conjecture

Arnold, 33, 55, 56

Hodge, 101

Seifert, 65

Weinstein, 65, 66

conjugation, 130

connectedness, 169, 174, 175

connection

flat, 159

form, 156

moduli space, 159

on a principal bundle, 155

space, 158

conormal

bundle, 17

space, 17

conservative system, 113

constrained system, 114

constraint set, 114

contact

contact structure on $S^{2 n-1}, 64$

dynamics, 63

element, 57, 61, 62

example of contact structure, 58

local contact form, 57

local normal form, 59

locally defining 1-form, 57

manifold, 57

point, 57

structure, 57

contactomorphism, 63

contractibility, 77

convexity, 169

cotangent bundle

canonical symplectomorphism, 11, 12

conormal bundle, 18

coordinates, 9

is a symplectic manifold, 9 
lagrangian submanifold, 16-18

projectivization, 59

sphere bundle, 59

zero section, 16

critical set, 174

curvature form, 157

D'Alembert

variational principle, 114

Darboux

chart, 7

theorem, 7, 45, 46

theorem for contact manifolds, 59

theorem in dimension two, 50

de Rham cohomology, 13, 39

deformation equivalence, 42

deformation retract, 40

Delzant

construction, 183, 185, 186

example of Delzant polytope, 177

example of non-Delzant polytope, 178

polytope, 177, 189

theorem, 179, 189

Dolbeault

cohomology, 81

theorem, 88

theory, 78

dual function, 122, 126

Duistermaat-Heckman

measure, 191

polynomial, 191, 192

theorem, 191, 194

dunce cap orbifold, 150

dynamical system, 33

effective

action, 170

moment map, 175

Ehresmann

connection, 156

$S^{4}$ is not an almost complex manifold, 76

embedding

closed, 15

coisotropic, 49, 53 definition, 15

isotropic, 53

lagrangian, 51

energy

classical mechanics, 107

energy-momentum map, 153

kinetic, 112, 113

potential, 112, 113

equations

Christoffel, 120

Euler-Lagrange, 105, 120, 123

Hamilton, 123, 148

Hamilton-Jacobi, 105

of motion, 113

equivariant

cohomology, 197

coisotropic embedding, 193

form, 197

moment map, 134

symplectic form, 198

tubular neighborhood theorem, 143

euclidean

distance, 24, 25

inner product, 24, 25

measure, 191

norm, 25

space, 24

Euler

Euler-Lagrange equations, 105, 116, 120,123

variational principle, 114

evaluation map, 128

exactly homotopic to the identity, 56

example

2-sphere, 97

coadjoint orbits, 137, 139

complex projective space, 89,103 , 181

complex submanifold of a Kähler manifold, 103

complex torus, 103

Delzant construction, 181

Fernández-Gotay-Gray, 102

Gompf, 103

hermitian matrices, 132

Hirzebruch surfaces, 178, 190 
Hopf surface, 102

Kodaira-Thurston, 102

McDuff, 43

non-singular projective variety, 95

of almost complex manifold, 76

of compact complex manifold, 101

of compact Kähler manifold, 96, 101

of compact symplectic manifold, 101

of complex manifold, 89

of contact manifold, 62

of contact structure, 58

of Delzant polytope, 177

of hamiltonian actions, 129

of infinite-dimensional symplectic manifold, 158

of Kähler submanifold, 95

of lagrangian submanifold, 16

of mechanical system, 113

of non-almost-complex manifold, 76

of non-Delzant polytope, 178

of reduced system, 153

of symplectic manifold, 6,9

of symplectomorphism, 22

oriented surfaces, 50

product of Kähler manifolds, 103

quotient topology, 135

reduction, 168

Riemann surface, 103

simple pendulum, 112

spherical pendulum, 152

Stein manifold, 103

Taubes, 103

toric manifold, 172

weighted projective space, 151

exponential map, 35

facet, 178

Fernández-Gotay-Gray example, 102

first Chern class, 194

first integral, 109

fixed point, 29, 33, 55

flat connection, 159

flow, 35

form

area, 50

canonical, 9, 10

complex-valued, 79

connection, 156

curvature, 157

de Rham, 6

Fubini-Study, 96, 168

harmonic, 98, 99

Kähler, 90, 98

Killing, 158

Liouville, 10

on a complex manifold, 85

positive, 92

symplectic, 6

tautological, 9, 10, 20

type, 79

free action, 135

Fubini theorem, 195

Fubini-Study form, 96, 168

function

biholomorphic, 89

dual, 122, 126

generating, 29

hamiltonian, 106, 134

$J$-holomorphic, 82

Morse-Bott, 174

stable, 121,125

strictly convex, 121,125

$G$-space, 134

gauge

group, 158, 159

theory, 155

transformation, 159

Gauss lemma, 28

generating function, 17, 22, 23, 29

geodesic

curve, 25

flow, 26, 27

geodesically convex, 25

minimizing, 25, 119, 120

Gompf construction, 103

Gotay

coisotropic embedding, 53

Fernández-Gotay-Gray, 102

gradient vector field, 107

gravitational potential, 113

gravity, 112, 152 
Gray

Gromov theorem (J. Gray), 59 group

gauge, 158, 159

Lie, 128

of symplectomorphisms, 12, 53

one-parameter group of diffeomorphisms, 127

product, 149

semisimple, 158

structure, 155

Guillemin

Atiyah-Guillemin-Sternberg theorem, 169

Hamilton equations, 23, 24, 107, 113, 123,148

Hamilton-Jacobi equations, 105

hamiltonian

action, 129, 130, 133, 164

function, 105, 106, 109, 134

$G$-space, 134

mechanics, 105

moment map, 134

reduced, 148

system, 109

vector field, 105, 106

harmonic form, 98, 99

Hausdorff quotient, 136

Heckman, see Duistermaat-Heckman

hermitian matrix, 132

hessian, 121, 125, 174

Hirzebruch surface, 178, 190

Hodge

complex Hodge theory, 99

conjecture, 101

decomposition, 98, 99

diamond, 101

number, 100

*-operator, 98

theorem, 98-100

theory, 98

$(J$-)holomorphic tangent vectors, 78 homotopy

definition, 40

formula, 39, 40

invariance, 39

operator, 40

Hopf

fibration, 64, 156

$S^{4}$ is not almost complex, 76

surface, 102

vector field, 64

immersion, 15

index, 174, 175

infinitesimal action, 156, 164

integrable

almost complex structure, 75,82

system, 109, 110, 171

integral

curve, 106, 113, 127

first, 109

of motion, 109, 147

intersection of lagrangian submanifolds, 55

inverse square law, 113

isometry, 120

isotopy

definition, 35

symplectic, 42

vs. vector field, 35

isotropic

embedding, 53

subspace, 8

isotropy, 135

$J$-anti-holomorphic function, 80

$(J$-)anti-holomorphic tangent vectors, 78

$J$-holomorphic curve, 82

$J$-holomorphic function, 80,82

$(J$-)holomorphic tangent vectors, 78

Jacobi

Hamilton-Jacobi equations, 105

identity, 108, 139

jacobiator, 139

Kähler

compact Kähler manifolds, 98 
form, 90, 98

local form, 94

manifold, 90, 98

potential, 93, 94

recipe, 92

submanifold, 94

Killing form, 158

kinetic energy, 112, 113

Kirillov

Kostant-Kirillov symplectic form, 139, Liouville

lift

Kodaira

complex surface, 103

complex surfaces, 102

Kodaira-Thurston example, 102

Kostant-Kirillov symplectic form, 139, 150 local form, 35, 94, 192

Lagrange

Euler-Lagrange equations, 120

variational principle, 114

lagrangian complement, 47

lagrangian fibration, 111

lagrangian submanifold

closed 1-form, 17

conormal bundle, 17, 18

definition, 16

generating function, 17, 23

intersection problem, 55

of $T^{*} X, 16$

vs. symplectomorphism, 15,19

zero section, 16

lagrangian subspace, 8, 46, 77

Laplace-Beltrami operator, 98

laplacian, 98

Lebesgue

measure, 191

volume, 191

left multiplication, 130

left-invariant, 130

Legendre

condition, 117

transform, 121, 122, 125, 126

Leibniz rule, 109, 139

Lie

algebra, 108, 130, 164

algebra cohomology, 165

bracket, 108

derivative, 36, 40

group, 128

Lie-Poisson symplectic form, 139, 150

of a diffeomorphism, 11

of a path, 115, 119

of a vector field, 106

linear momentum, 137

Arnold-Liouville theorem, 110

form, 10

measure, 191

torus, 110

volume, 13

locally free action, 135

manifold

almost symplectic, 74

complex, 83

infinite-dimensional, 158

Kähler, 90, 98

of contact elements, 61

of oriented contact elements, 62

riemannian, 119

symplectic, 6

toric, see toric manifold

with corners, 188

Marsden-Weinstein-Meyer

quotient, 141

theorem, 136, 141

Maupertius

variational principle, 114

McDuff counterexample, 43

measure

Duistermaat-Heckman, 191

Lebesgue, 191

Liouville, 191

symplectic, 191

mechanical system, 113

mechanics

celestial, 33

classical, 107

metric, 24, 70, 119

Meyer, see Marsden-Weinstein-Meyer 
minimizing

action, 115

locally, 115, 117

property, 117

moduli space, 159

moment map

actions, 127

definition, 133

effective, 175

equivariance, 134

example, 162

existence, 164, 166

hamiltonian $G$-space, 134

in gauge theory, 155

origin, 127

uniqueness, 164, 167

upgraded hamiltonian function, 130

moment polytope, 169

momentum, 107, 123, 137

momentum vector, 137

Morse

Morse-Bott function, 174

Morse function, 55

Morse theory, 55, 169

Moser

equation, 44

theorem - relative version, 45

theorem - version I, 43

theorem - version II, 44

trick, 42-44, 50

motion

constant of motion, 109

equations, 113

integral of motion, 109, 147

neighborhood

convex, 37

$\varepsilon$-neighborhood theorem, 38

tubular neighborhood, 51

tubular neighborhood fibration, 39

tubular neighborhood in $\mathbb{R}^{n}, 41$

tubular neighborhood theorem, 37

Weinstein lagrangian neighborhood, 46,48

Weinstein tubular neighborhood, 51 Newlander-Nirenberg theorem, 82, 88
Newton

polytope, 177

second law, 105, 107, 113, 114

Nijenhuis tensor, 82, 88

Nikodym

Radon-Nikodym derivative, 191

Nirenberg

Newlander-Nirenberg theorem, 82

Noether

principle, 127,147

theorem, 147

non-singular projective variety, 95

nondegenerate

bilinear map, 4

fixed point, 55

normal

bundle, 37, 41

space, $37,41,51$

number

Betti, 100

Hodge, 100

one-parameter group of diffeomorphisms, 127

operator

Laplace-Beltrami, 98

orbifold

conehead, 150

dunce cap, 150

examples, 150

reduced space, 150

teardrop, 150

orbit

definition, 135

point-orbit projection, 135

space, 135

topology of the orbit space, 135

unstable, 135

oriented surfaces, 50

overtwisted contact structure, 66

pendulum

simple, 112, 114

spherical, 152

periodic point, 29

phase space, 107, 113, 148 
Picard theorem, 36

Poincaré

last geometric theorem, 33

Poincaré-Birkhoff theorem, 33

recurrence theorem, 32

point-orbit projection, 135

Poisson

algebra, 109

bracket, 108, 110, 134, 164

Lie-Poisson symplectic form, 139, 150

structure on $\mathfrak{g}^{*}, 139$

polar decomposition, 69, 70

polytope

Delzant, 177, 189

example of Delzant polytope, 177

example of non-Delzant polytope, 178

facet, 178

moment, 169

Newton, 177

rational, 177

simple, 177

smooth, 177

positive

form, 92

inner product, 24,77

vector field, 66

potential

energy, 112, 113

gravitational, 113

Kähler, 93, 94

strictly plurisubharmonic, 92

primitive vector, 178

principal bundle

connection, 155

gauge group, 158

principle

Noether, 127, 147

of least action, 114

variational, 114

product group, 149

projectivization, 61

proper function, 15, 103, 121

pseudo-holomorphic curve, 67,82

pullback, 7 quadratic growth at infinity, 126

quadrature, 153

quotient

Hausdorff, 136

Marsden-Weinstein-Meyer, 141

symplectic, 141

topology, 135

Radon-Nikodym derivative, 191

rational polytope, 177

recipe

for Kähler forms, 92

for symplectomorphisms, 22

recurrence, 29, 32

reduced

hamiltonian, 148

phase space, 148

space, 136, 141, 150

reduction

example, 168

for product groups, 149

in stages, 149

local form, 192

low-brow proof, 141

Noether principle, 147

other levels, 149

preview, 136

reduced space, 136

symmetry, 147

Reeb vector field, 63

representation

adjoint, 130, 131

coadjoint, 130, 131

of a Lie group, 128

retraction, 40

Riemann

Cauchy-Riemann equations, 84

surface, 103, 158

riemannian

distance, 25

manifold, 24, 119

metric, 24, 49, 70, 119

right multiplication, 130

right-invariant, 130 
s.p.s.h., 92

Seiberg-Witten invariants, 103

Seifert conjecture, 65

semisimple, 158, 167

simple pendulum, 112

simple polytope, 177

skew-symmetric bilinear map

nondegenerate, 4

rank, 4

standard form, 3

symplectic, 4

skew-symmetry

definition, 3

forms, 13

standard form for bilinear maps, 3

slice theorem, 144

smooth polytope, 177

space

affine, 158

configuration, 107, 113

moduli, 159

normal, 41, 51

of connections, 158

phase, 107, 113

total, 155

spherical pendulum, 152

splittings, 78

stability

definition, 121

set, 122

stabilizer, 135

stable

function, 125

point, 112, 152

Stein manifold, 103

stereographic projection, 89, 97

Sternberg

Atiyah-Guillemin-Sternberg theorem, 169

Stokes theorem, 13, 161

strictly convex function, $117,121,125$

strictly plurisubharmonic, 92

strong isotopy, 42, 50

submanifold, 15

submanifold

almost complex, 76
Kähler, 94

subspace

coisotropic, 8

isotropic, 5,8

lagrangian, 8, 46, 77

symplectic, 5, 8

supercommutativity, 197

superderivation, 197

symplectic

action, 129

almost symplectic manifold, 74

basis, 5

bilinear map, 4

blowup, 189

canonical symplectic form on a coadjoint orbit, 139, 150, 162

cotangent bundle, 9

deformation equivalence, 42

duality, 5

equivalence, 42

equivariant form, 198

form, 6, 13

Fubini-Study form, 168

isotopy, 42

linear algebra, 8, 51

linear group, 72

linear symplectic structure, 4

manifold, 6

measure, 191

normal forms, 46

orthogonal, 8

properties of linear symplectic structures, 5

quotient, 141

reduction, see reduction

strong isotopy, 42

structure on the space of connections, 158

subspace, 8

toric manifold, see toric manifold

vector bundle, 74

vector field, 105, 106, 129

vector space, 4

volume, 13, 191, 195

symplectization, 64

symplectomorphic, 5, 42 
symplectomorphism

Arnold conjecture, 33, 55

canonical, 12

definition, 7

equivalence, 15

exactly homotopic to the identity, 56

fixed point, 33, 55

generating function, 23

group of symplectomorphisms, 12 , 53

linear, 5

recipe, 22

tautological form, 20

vs. lagrangian submanifold, 15, 18, 19

system

conservative, 113

constrained, 114

mechanical, 113

Taubes

$\mathbb{C P}^{2} \# \mathbb{C P}^{2} \# \mathbb{C P}^{2}$ is not complex, 103 unique symplectic structure on $\mathbb{C P}^{2}$, 103

tautological form on $T^{*} X$

coordinate definition, 9, 10

intrinsic definition, 10

naturality, 11

property, 10

symplectomorphism, 20

teardrop orbifold, 150

theorem

Archimedes, 192

Arnold-Liouville, 110

Atiyah-Guillemin-Sternberg, 136, 169

Banyaga, 92

coisotropic embedding, 49

convexity, 169

Darboux, 7, 46, 50

Delzant, 136, 179, 189

Dolbeault, 88

Duistermaat-Heckman, 191, 194

$\varepsilon$-neighborhood, 38

equivariant coisotropic embedding, 193
Euler-Lagrange equations, 123

Fubini, 195

Gray, 59

Hodge, 98-100

implicit function, 23

local normal form for contact manifolds, 59

Marsden-Weinstein-Meyer, 136, 141

Moser - relative version, 45

Moser - version I, 43

Moser - version II, 44

Newlander-Nirenberg, 82, 88

Noether, 147

Picard, 36

Poincaré recurrence, 32

Poincaré's last geometric theorem, 33

Poincaré-Birkhoff, 33

slice, 144

standard form for skew-symmetric bilinear maps, 3

Stokes, 13, 161

symplectomorphism vs. lagrangian submanifold, 19

tubular neighborhood, 37, 51

tubular neighborhood in $\mathbb{R}^{n}, 41$

Weinstein lagrangian neighborhood, 46,48

Weinstein tubular neighborhood, 51

Whitehead lemmas, 167

Whitney extension, 48

Thurston

Kodaira-Thurston example, 102

tight contact structure, 66

time-dependent vector field, 35

ological constraint, 100

topology of the orbit space, 135

toric manifold

classification, 177

definition, 171, 177

example, 172

4-dimensional, 189

total space, 155

transitive action, 135

tubular neighborhood

equivariant, 143 
fibration, 39

Young inequality, 126

homotopy-invariance, 39

in $\mathbb{R}^{n}, 41$

theorem, 37, 51

Weinstein theorem, 51

twisted product form, 19

twisted projective space, 151

unique symplectic structure on $\mathbb{C P}^{2}, 103$

unstable

orbit, 135

point, 112, 152

variational

principle, 113, 114

problem, 113, 122

vector field

complete, 129

gradient, 107

hamiltonian, 105, 106

Lie algebra, 164

symplectic, 105, 106, 129

vector space

complex, 68

symplectic, 4

velocity, 119

volume, 13, 191, 195

weighted projective space, 151

Weinstein

conjecture, 65,66

isotropic embedding, 53

lagrangian embedding, 51

lagrangian neighborhood theorem, 46, 48

Marsden-Weinstein-Meyer quotient, 141

Marsden-Weinstein-Meyer theorem, 141

tubular neighborhood theorem, 51

Whitehead lemmas, 167

Whitney extension theorem, 48

Wirtinger inequality, 117, 118

Witten

Seiberg-Witten invariants, 103

work, 113 